\title{
NAVIGATION QUALITY \\ FROM LAKE BAIKAL 1992 MULTICHANNEL SEISMICS CRUISE
}

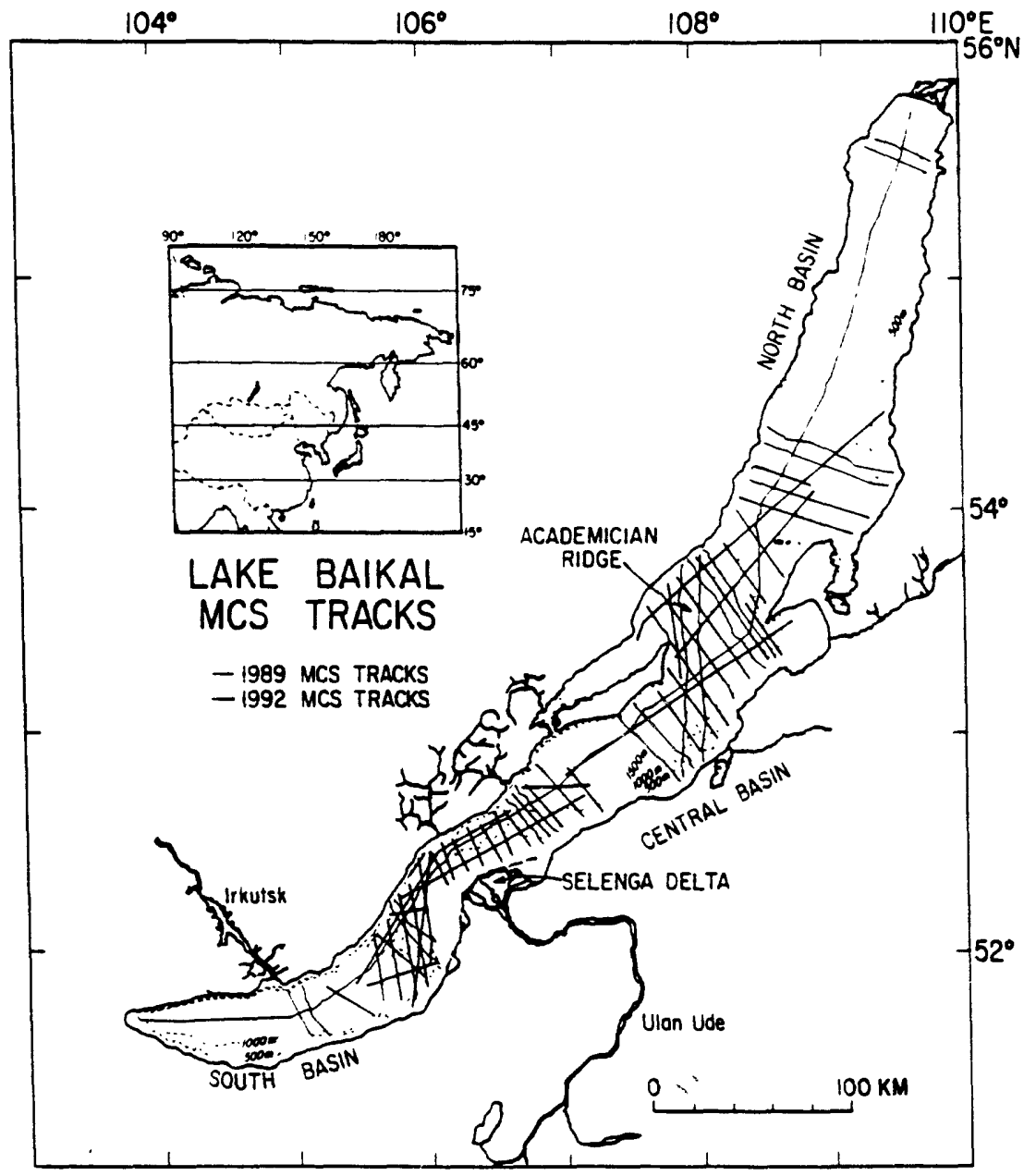

by

Deborah R. Hutchinson

U.S. Geological Survey

Woods Hole, MA 02543

Open-File Report \#95-233

1995

This report is preliminary and has not been reviewed for conformity with U.S. Geological Survey editorial standards and nomenclature. Use of trade names is for purposes of identification only and does not constitute endorsement by the U.S. Geological Survey. 


\section{TABLE OF CONTENTS}

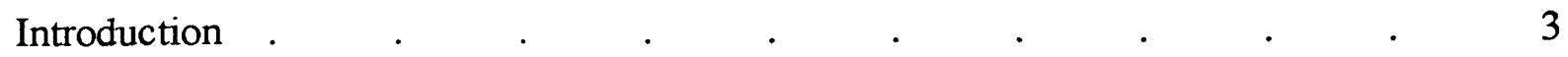

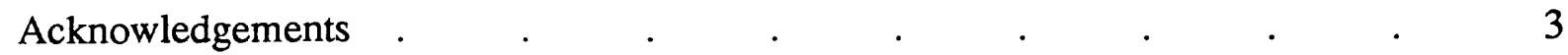

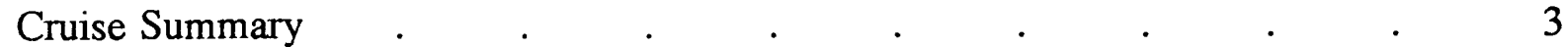

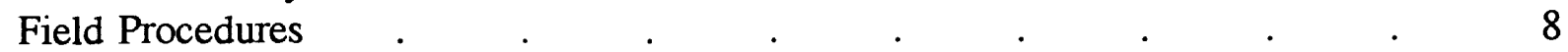

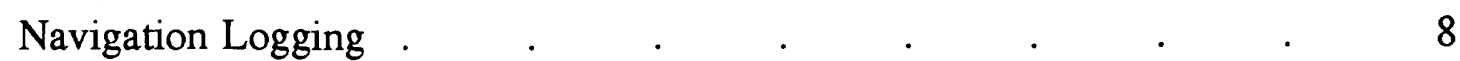

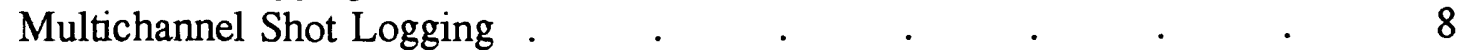

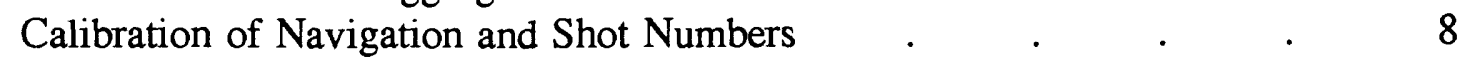

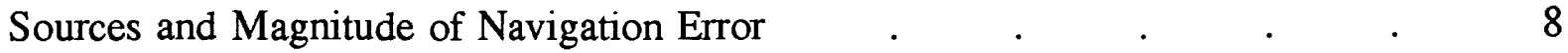

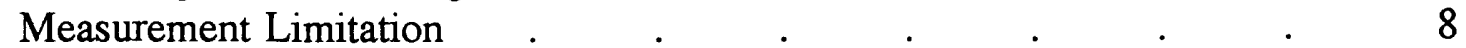

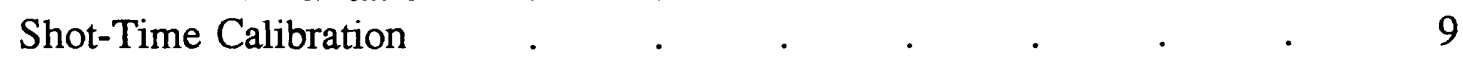

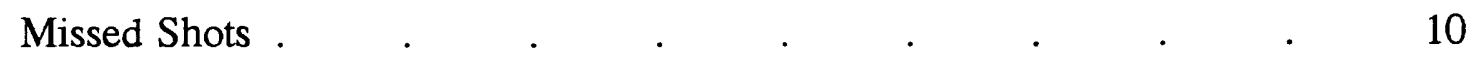

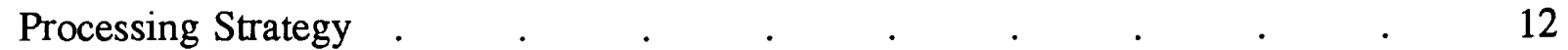

Results . . . . . . . . . . . . . . 15

Quality of the Raw Navigation $\quad$. $\quad$. $\quad$. $\quad$. $\quad$. $\quad$. 15

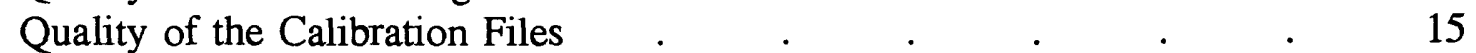

Quality of the Distance Between Shots . $\quad . \quad$. $\quad . \quad$. 16

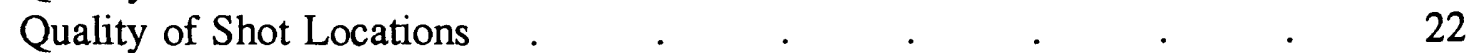

Discussion

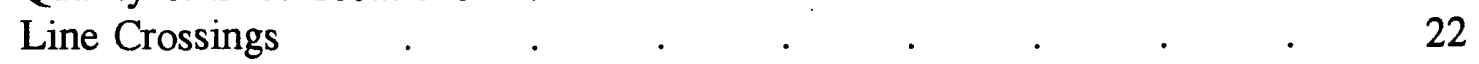

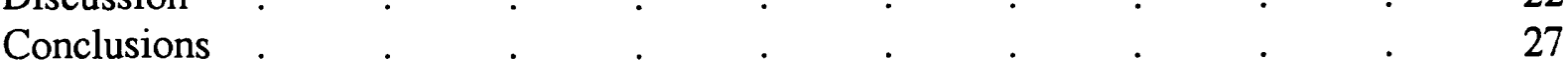

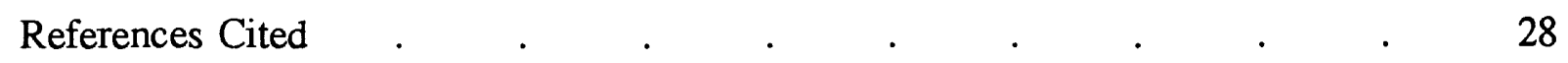

Appendix 1: Plots of Raw GPS Navigation $\quad$. $\quad$. $\quad$. $\quad$. $\quad$. 29

Appendix 2: Plots of Shot-Time Calibration and Shot-Distance Information . 101

Appendix 3: Line Crossings . . . . . . . . . 158 


\section{INTRODUCTION}

In August-September, 1992, a comprehensive multichannel seismic-reflection survey was conducted in Lake Baikal, Siberia (Figure 1), by U.S. and Russian scientists (Klitgord et al., 1993). Navigation utilized U.S.-supplied Global Positioning Satellite (GPS) receivers. Pre-cruise uncertainty in the availability and quality of the GPS positions at Lake Baikal together with limitations in both the ships layout and the navigation software resulted in (a) the seismic source being fired by increments of time rather than increments of distance and (b) navigation being acquired independently of the multichannel shot data. Because the navigation and multichannel data were not linked, the challenge in processing the shot point data was to assign an accurate time to each shot so that its position could be estimated from the raw navigation data.

The purpose of this report is to describe how times for each shot were derived, to describe how the positions were established for each shot number; and to assign a quality factor to the navigation for each line. The implications of these results for the geometry and processing of the multichannel seismic data are also discussed. This report complements other reports about the scientific objectives and operations of the cruise (Klitgord et al., 1993; Nichols et al., 1992) and the processing of the multichannel seismic profiles (Agena et al., 1994).

\section{ACKNOWLEDGEMENTS}

The success of the field program is due to the combined efforts of Russian and American scientists who participated in the R/V Balkhash multichannel cruise (Klitgord et al., 1993). I thank Warren Agena for his detailed accounting of missed shots based on observers logs and processing experience, Chris Schneider for developing the initial Matlab routines for displaying the navigation information, and Chuck Denham for advice and guidance in developing subsequent Matlab routines. Discussions with Barry Irwin, Uri ten Brink, and Chris Schneider helped focus many of the analyses presented here. Constructive reviews by Barry Irwin and Dave Foster are appreciated.

\section{CRUISE SUMMARY}

Approximately $2,250 \mathrm{~km}$ of multichannel seismic profiles were collected aboard $\mathrm{R} / \mathrm{V}$ Balkhash in Lake Baikal in 1992 (Figure 1). Line numbers were assigned based on whether the lines were dip lines across the lake (even numbers) or tie lines along the lake (odd numbers). Because unequal numbers of dip and tie lines were shot, the line numbers are not sequential. There are 42 separate lines with numbers which range from 1 to 60 . An additional 6 segments comprise reshot lines (3A, 10A, 17A, 40A, 50A, and 50B). Hence there are a total of 48 line segments for purposes of processing. The reshot lines have been given numerical designations of 61 - 66 (Table 1) for the purposes of navigation processing in this report but should otherwise be labelled by their A and B names officially, as shown on Plate 1. Line 17A (66) is a reshoot of line 17 with large-volume air guns and a slower firing rate (120 seconds) because of coincident recording of Ocean Bottom Seismometer data (ten Brink et al., 1993). The other A and $\mathrm{B}$ lines were reshot because of acquisition or navigation errors and are continuations of the original lines. 


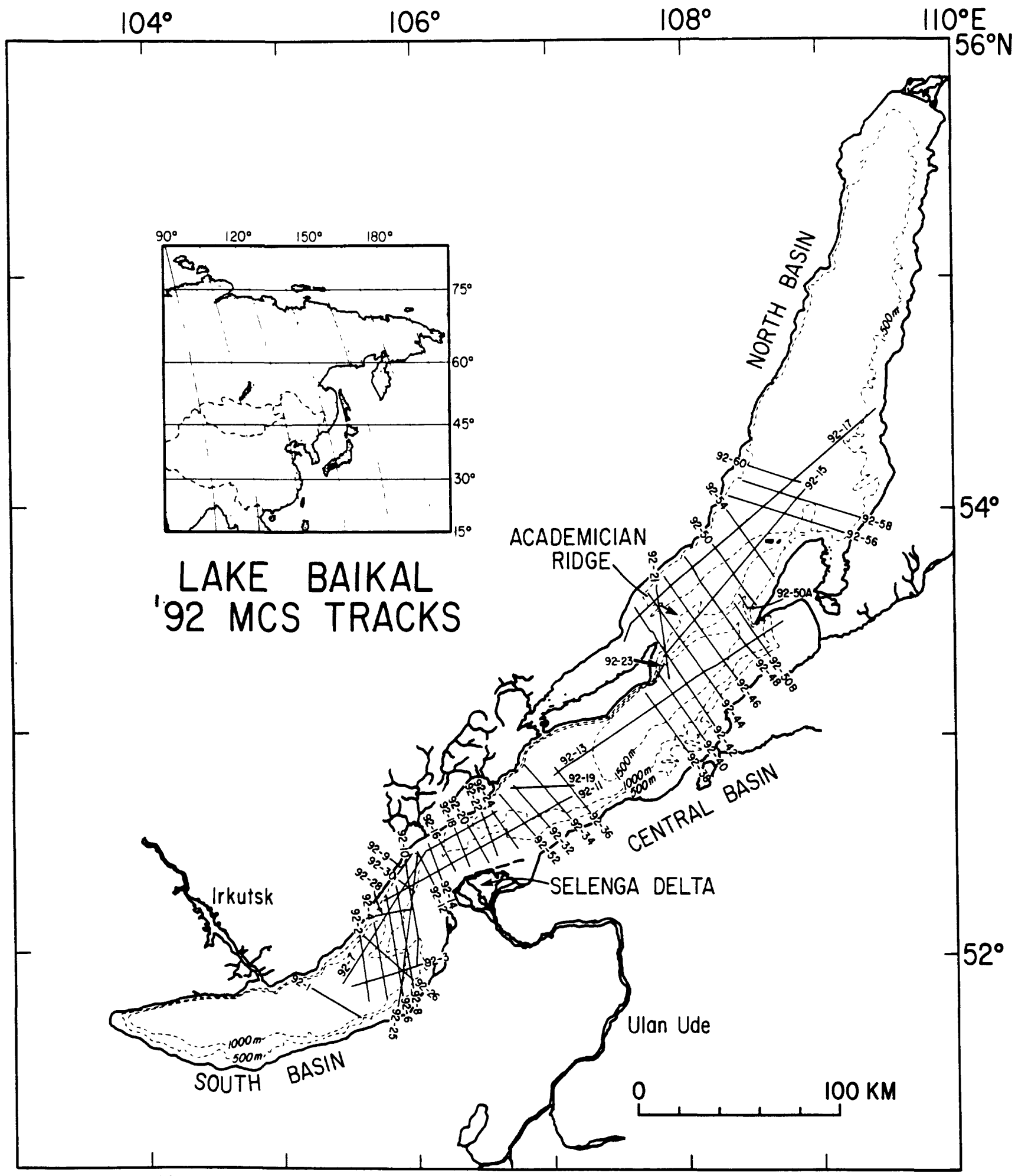

MERCATOR PROJECTION

Figure 1. Map of Lake Baikal showing locations of 1992 multichannel seismic reflection lines. Detailed shot point map shown with locations of shot-time calibration points is shown at 1:1,000,000 in Plate 1. 


\begin{tabular}{|c|c|c|c|c|c|c|c|c|c|c|c|c|c|c|c|c|c|c|c|c|}
\hline \multirow{5}{*}{ 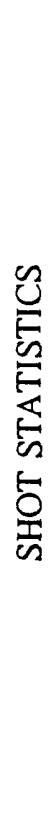 } & 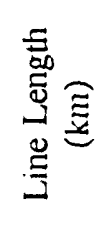 & 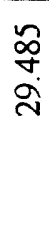 & $\begin{array}{l}\stackrel{2}{5} \\
\stackrel{0}{m} \\
\text {. }\end{array}$ & $\begin{array}{l}\infty \\
2 \\
\Sigma\end{array}$ & $\begin{array}{l}\text { gे } \\
\text { jे }\end{array}$ & $\frac{\widetilde{\sigma}}{\tilde{\sigma}}$ & $\begin{array}{l}\text { J్ } \\
\text { ஸे }\end{array}$ & $\begin{array}{l}\stackrel{n}{\sim} \\
\underset{n}{\tilde{n}}\end{array}$ & 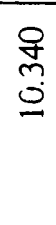 & $\begin{array}{l}\vec{\delta} \\
\infty \\
\text { mi }\end{array}$ & $\begin{array}{l}8 \\
\stackrel{8}{8} \\
8\end{array}$ & $\begin{array}{l}\stackrel{\rho}{n} \\
\infty \\
\infty\end{array}$ & $\begin{array}{l}\tilde{J} \\
\stackrel{n}{m}\end{array}$ & $\frac{\mathbb{Z}}{8}$ & $\begin{array}{l}\text { षे } \\
\infty \\
0\end{array}$ & $\begin{array}{l}\infty \\
\stackrel{+}{+}\end{array}$ & $\begin{array}{l}\overline{5} \\
0 \\
0\end{array}$ & $\begin{array}{l}\text { 응 } \\
\text { ᄋ }\end{array}$ & $\begin{array}{l}\text { P } \\
\text { న } \\
\text { లn }\end{array}$ & $\begin{array}{l}\widetilde{\delta} \\
\infty \\
\text { ర్ల }\end{array}$ \\
\hline & 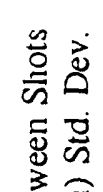 & o. & $\stackrel{\circ}{\circ}$ & $\underset{n}{\tilde{n}}$ & $\stackrel{a}{m}$ & $\hat{a}$ & $\stackrel{r}{i}$ & $\stackrel{9}{0}$ & $\bar{\sigma}$ & $\stackrel{m}{\circ}$ & $\stackrel{\infty}{\sim}$ & $\underset{\infty}{0}$ & $\stackrel{b}{i}$ & $\stackrel{\infty}{\sim}$ & $\stackrel{\circ}{\dot{\gamma}}$ & $\hat{m}$ & $\stackrel{0}{r}$ & aे & an & $\stackrel{q}{+}$ \\
\hline & 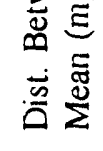 & $\begin{array}{l}\dot{\$} \\
\text { in } \\
\text { n. }\end{array}$ & 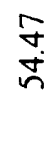 & $\frac{\widetilde{v}}{\sigma}$ & $\stackrel{\infty}{\stackrel{\infty}{n}}$ & $\begin{array}{l}\stackrel{2}{n} \\
\stackrel{n}{n}\end{array}$ & $\begin{array}{l}\stackrel{\nabla}{\sim} \\
\stackrel{\circ}{n}\end{array}$ & 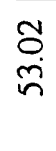 & $\begin{array}{l}\tilde{\alpha} \\
\sigma\end{array}$ & $\begin{array}{l}m \\
\tilde{n} \\
n\end{array}$ & ô & \begin{tabular}{l}
$\infty$ \\
$\infty$ \\
\multirow{\sigma}{*}{}
\end{tabular} & $\frac{\bar{n}}{n}$ & $\stackrel{\tilde{n}}{\mathfrak{v}}$ & $\frac{8}{n}$ & $\frac{d}{\stackrel{d}{\sigma}}$ & 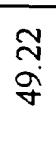 & $\stackrel{\text { ğ }}{g}$ & $\underset{\sim}{\tilde{N}}$ & $\frac{2}{\stackrel{8}{n}}$ \\
\hline & 气̆ & ฉૂ & $\tilde{b}$ & $\hat{\text { p}}$ & $\stackrel{\infty}{\sim}$ & ธิ & $\stackrel{\text { ণ্ণ }}{\underset{N}{ }}$ & 8 & ᄋ్ & $\frac{N}{0}$ & $\stackrel{\curvearrowleft}{\approx}$ & 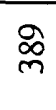 & 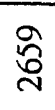 & $\mathscr{f}$ & $\stackrel{\infty}{\equiv}$ & $\stackrel{n}{m}$ & ঙ্ণি & $\frac{a}{v}$ & 요 & $\frac{N}{\nabla}$ \\
\hline & $\bar{s}$ & 气̊ & $\hat{\widehat{b}}$ & $\sqrt[n]{n}$ & $\stackrel{\infty}{\sim}$ & ळ & 유 & §̊ & ర్ల & $\frac{\pi}{6}$ & $\stackrel{\grave{n}}{\grave{N}}$ & 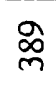 & $\stackrel{\text { }}{\curvearrowright}$ & $\mathscr{f}$ & $\tilde{\swarrow}$ & $\stackrel{n}{n}$ & 유 & $\frac{a}{\gamma}$ & $\frac{\nabla}{6}$ & $\stackrel{\stackrel{N}{\nabla}}{7}$ \\
\hline \multirow{3}{*}{ 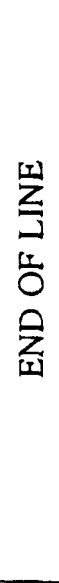 } & 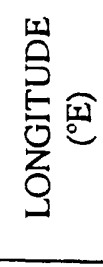 & 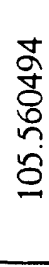 & 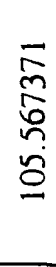 & 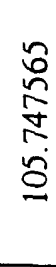 & 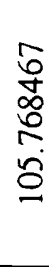 & $\begin{array}{l}\bar{N} \\
\overline{8} \\
0 \\
\vdots \\
0\end{array}$ & 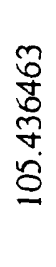 & 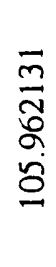 & \begin{tabular}{l}
$\frac{9}{5}$ \\
\multirow{5}{5}{} \\
ปे
\end{tabular} & 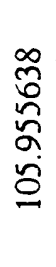 & 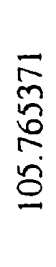 & $\frac{\stackrel{0}{\infty}}{\stackrel{\infty}{0}}$ & $\begin{array}{l}\bar{a} \\
\frac{0}{0} \\
\text { ஸ் }\end{array}$ & 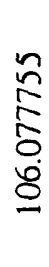 & $\begin{array}{l}\text { స్ } \\
\infty \\
\infty \\
\infty \\
\infty \\
0\end{array}$ & 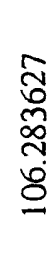 & 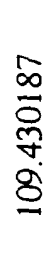 & 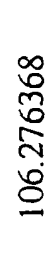 & $\begin{array}{l}8 \\
8 \\
8 \\
0 \\
5 \\
0\end{array}$ & 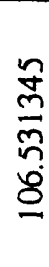 \\
\hline & 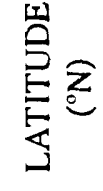 & 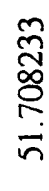 & $\begin{array}{l}\vec{n} \\
0 \\
\infty \\
0 \\
\text { i } \\
\text { n. }\end{array}$ & $\begin{array}{l}\text { N } \\
\text { ू̆ } \\
\frac{\infty}{n}\end{array}$ & 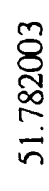 & 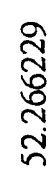 & 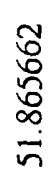 & 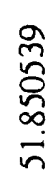 & $\begin{array}{l}\frac{0}{8} \\
8 \\
\frac{n}{5} \\
\text { v }\end{array}$ & 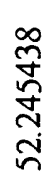 & 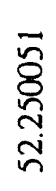 & $\begin{array}{l}\stackrel{P}{N} \\
\stackrel{\infty}{\sim} \\
\stackrel{\sim}{\sim} \\
\stackrel{n}{n}\end{array}$ & $\begin{array}{l}\text { ฟิ } \\
\text { చั } \\
\infty \\
\text { ๙n }\end{array}$ & $\begin{array}{l}\frac{\nabla}{\sigma} \\
\frac{\pi}{n} \\
\frac{n}{n}\end{array}$ & 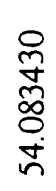 & 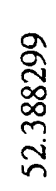 & $\frac{8}{\frac{8}{+}}$ & \begin{tabular}{l}
$\bar{O}$ \\
$\infty$ \\
$\infty$ \\
\multirow{2}{n}{} \\
ñ
\end{tabular} & 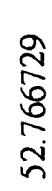 & $\begin{array}{l}\bar{n} \\
8 \\
\stackrel{n}{ \pm} \\
\text { ป } \\
\text { n. }\end{array}$ \\
\hline & ह & กิ & $\tilde{\widehat{b}}$ & n & $\stackrel{\infty}{\infty}$ & \& & $\underset{\sim}{\stackrel{ர}{\sim ~}}$ & §ิ & 유 & $\frac{7}{6}$ & $\hat{\frac{n}{2}}$ & ஓ & $\stackrel{\mathscr{ٌ}}{\sim}$ & $\mathscr{f}$ & 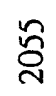 & $\stackrel{n}{m}$ & 율 & $\frac{a}{\gamma}$ & $\frac{\nabla}{\sigma}$ & $\stackrel{\mathcal{F}}{\vec{V}}$ \\
\hline \multirow{3}{*}{ 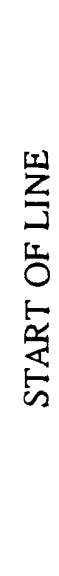 } & 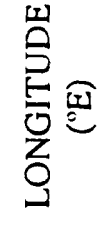 & 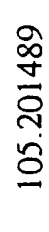 & 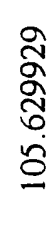 & 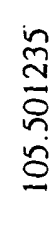 & $\begin{array}{l}\frac{m}{8} \\
\delta \\
\delta \\
0 \\
\dot{0}\end{array}$ & $\begin{array}{l}\hat{2} \\
2 \\
2 \\
\infty \\
\text { n. } \\
0\end{array}$ & 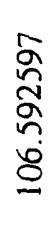 & 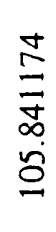 & 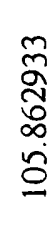 & 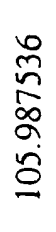 & 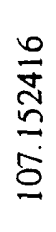 & 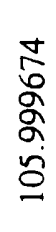 & 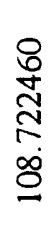 & $\begin{array}{l}\bar{N} \\
\frac{\pi}{8} \\
\frac{0}{0}\end{array}$ & $\begin{array}{l}\underset{N}{N} \\
\stackrel{N}{\infty} \\
\vdots \\
\vdots \\
0\end{array}$ & $\begin{array}{l}\circ \\
2 \\
2 \\
\check{2} \\
0\end{array}$ & \begin{tabular}{l}
8 \\
0 \\
0 \\
\multirow{2}{n}{} \\
$\vdots$ \\
0
\end{tabular} & 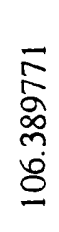 & 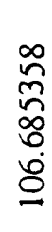 & 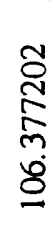 \\
\hline & 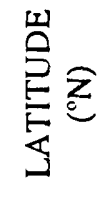 & 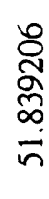 & $\begin{array}{l}\overline{\hat{O}} \\
\stackrel{\infty}{0} \\
\frac{\bar{n}}{n}\end{array}$ & $\begin{array}{l}n \\
\delta \\
\delta \\
\infty \\
i \\
i\end{array}$ & $\begin{array}{l}\frac{n}{8} \\
\text { จे } \\
\text { กี่ }\end{array}$ & $\frac{\infty}{\frac{\infty}{8}}$ & 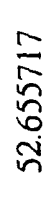 & 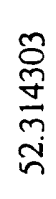 & 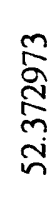 & 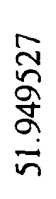 & 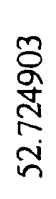 & 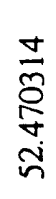 & 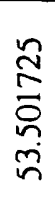 & 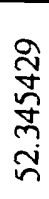 & 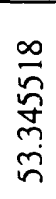 & $\begin{array}{l}\frac{d}{5} \\
\stackrel{+}{\sim} \\
\text { ñ } \\
\text { ñ }\end{array}$ & 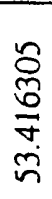 & 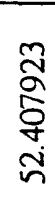 & 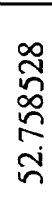 & 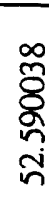 \\
\hline & $\bar{s}$ & - & - & - & $\rightarrow$ & - & - & - & $N$ & - & - & - & - & - & - & - & $\forall$ & - & - & - \\
\hline & : & - & $\sim$ & $m$ & $\nabla$ & 0 & $r$ & $\infty$ & $a$ & 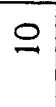 & $=$ & $\simeq$ & $\stackrel{m}{2}$ & $\Xi$ & $\simeq$ & $\underline{0}$ & 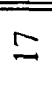 & $\infty$ & a & శి \\
\hline
\end{tabular}




\begin{tabular}{|c|c|c|c|c|c|c|c|c|c|c|c|c|c|c|c|c|c|c|c|c|c|c|}
\hline \multirow{5}{*}{ 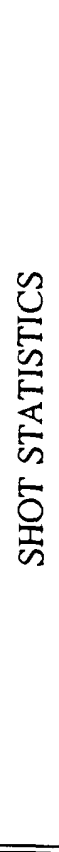 } & 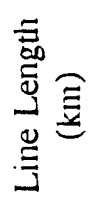 & $\begin{array}{l}\text { ठे } \\
\infty \\
\text { bे }\end{array}$ & $\begin{array}{l}8 \\
\dot{0} \\
\grave{v}\end{array}$ & $\begin{array}{l}\Xi \\
\Xi\end{array}$ & $\begin{array}{l}\text { ֶ̊ } \\
\text { } \\
\text { }\end{array}$ & $\frac{\bar{a}}{\infty}$ & 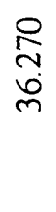 & $\begin{array}{l}\stackrel{N}{\infty} \\
\stackrel{\infty}{ }\end{array}$ & $\frac{0}{a}$ & $\begin{array}{l}\stackrel{0}{0} \\
\text { ㅁ. } \\
\text { त }\end{array}$ & $\begin{array}{l}\tilde{n} \\
\tilde{n} \\
\tilde{m}\end{array}$ & $\begin{array}{l}\overline{\bar{\sigma}} \\
\infty \\
\stackrel{\sim}{0}\end{array}$ & 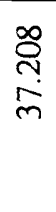 & 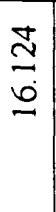 & 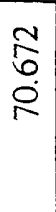 & $\begin{array}{l}\text { ठ } \\
\text { ભે } \\
\text { గి }\end{array}$ & 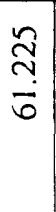 & $\begin{array}{l}\bar{m} \\
\stackrel{0}{8}\end{array}$ & $\begin{array}{l}\text { aे } \\
\infty \\
\text { b } \\
\text { p. }\end{array}$ & 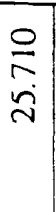 & $\frac{\mathcal{P}}{\dot{P}}$ & $\begin{array}{l}\infty \\
\underset{\infty}{\infty} \\
\infty \\
n\end{array}$ \\
\hline & 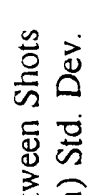 & $\stackrel{ }{O}$ & $\stackrel{m}{\infty}$ & $\stackrel{0}{m}$ & $\begin{array}{l}0 \\
\dot{n}\end{array}$ & $\hat{\infty}^{\circ}$ & $\begin{array}{l}\infty \\
\stackrel{\infty}{ }\end{array}$ & $\stackrel{\nabla}{b}$ & $m$ & $\begin{array}{l}\infty \\
\mathfrak{v}\end{array}$ & $n$ & $\underset{\infty}{N}$ & $\vec{i}$ & $\stackrel{n}{=}$ & $\ddot{\nabla}$ & $\underset{\sim}{\infty}$ & $\vec{\sim}$ & $\stackrel{\sim}{n}$ & $\begin{array}{l}0 \\
\dot{n}\end{array}$ & â & $\hat{m}$ & $\stackrel{m}{m}$ \\
\hline & 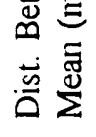 & $\begin{array}{l}\hat{a} \\
\hat{n}\end{array}$ & $\begin{array}{l}\underset{q}{q} \\
\text { Ṅ}\end{array}$ & $\bar{\sim}$ & $\begin{array}{l}\infty \\
\infty \\
\infty\end{array}$ & $\frac{n}{n}$ & $\begin{array}{l}\text { ָ̊ } \\
\text { nn }\end{array}$ & \begin{tabular}{l}
8 \\
\multirow{\sigma}{*}{} \\
\end{tabular} & $\stackrel{\curvearrowright}{n}$ & $\frac{n}{n}$ & $\begin{array}{l}\infty \\
\stackrel{2}{\circ} \\
\stackrel{n}{n}\end{array}$ & $\frac{\infty}{n}$ & $\hat{a}$ & $\frac{\infty}{b}$ & $\begin{array}{l}\mathbb{N} \\
\sim\end{array}$ & $\frac{\infty}{n}$ & $\frac{n}{n}$ & $\begin{array}{l}\check{\nabla} \\
\dot{v}\end{array}$ & $\begin{array}{l}\overrightarrow{7} \\
\dot{n}\end{array}$ & $\begin{array}{l}\bar{\infty} \\
\stackrel{0}{n} \\
\text { n. }\end{array}$ & $\begin{array}{l}\stackrel{g}{ \pm} \\
\stackrel{\circ}{n}\end{array}$ & $\frac{0}{0}$ \\
\hline & : & ભి & $\vec{z}$ & $\underset{\sim}{\Delta}$ & ஜூo & $\stackrel{\circ}{n}$ & ర్రం & $\underset{\mathcal{Y}}{\widetilde{J}}$ & \pm & $\widehat{\widetilde{N}}$ & $\begin{array}{l}\infty \\
8 \\
8\end{array}$ & ले & $\vec{r}$ & $\underset{\sim}{\infty}$ & $\vec{\varpi}$ & ๗ે & $\stackrel{\unrhd}{\equiv}$ & $\stackrel{8}{g}$ & \&్రి & so & ஜ̊ & $\underline{0}$ \\
\hline & $\bar{s}$ & 命 & $\overrightarrow{7}$ & $\frac{\Delta}{\sim}$ & $\hat{\sigma}$ & $\frac{\sim}{n}$ & ర్రి & $\underset{\Im}{\widetilde{\mho}}$ & \pm & $\frac{n}{n}$ & $\underset{0}{\mathscr{0}}$ & $\stackrel{\sim}{\sim}$ & 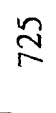 & $\underset{\sim}{\infty}$ & $\overline{\widetilde{N}}$ & 오 & $\stackrel{\infty}{=}$ & $\stackrel{g}{\varrho}$ & \&్రి & $\stackrel{n}{q}$ & $\underset{\substack{\infty \\
N}}{\infty}$ & $\stackrel{\text { g }}{=}$ \\
\hline \multirow{3}{*}{ 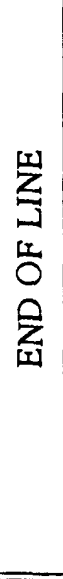 } & 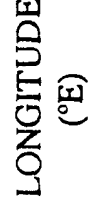 & $\begin{array}{l}8 \\
\infty \\
\infty \\
\infty \\
\infty \\
5 \\
0\end{array}$ & 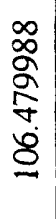 & $\frac{n}{\stackrel{n}{\Xi}}$ & 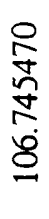 & $\begin{array}{l}\text { n } \\
\text { Oे } \\
\stackrel{+}{5} \\
\infty \\
\stackrel{0}{0}\end{array}$ & 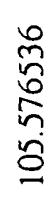 & $\begin{array}{l}\frac{n}{a} \\
\frac{n}{\alpha} \\
\vdots \\
\vdots\end{array}$ & 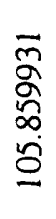 & $\begin{array}{l}0 \\
\frac{0}{0} \\
5 \\
a \\
8 \\
0\end{array}$ & \begin{tabular}{l}
\multirow{2}{0}{} \\
$\frac{1}{5}$ \\
2 \\
8 \\
0
\end{tabular} & 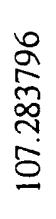 & $\begin{array}{l}8 \\
0 \\
\infty \\
0 \\
0 \\
5 \\
0\end{array}$ & $\begin{array}{l}0 \\
\infty \\
\infty \\
\infty \\
\infty \\
5 \\
0\end{array}$ & 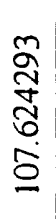 & 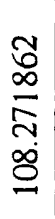 & 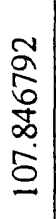 & $\begin{array}{l}n \\
\infty \\
\infty \\
8 \\
\infty \\
0 \\
0\end{array}$ & 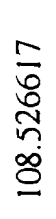 & $\begin{array}{l}\qquad \\
0 \\
8 \\
0 \\
\varnothing \\
8 \\
0\end{array}$ & 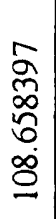 & 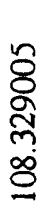 \\
\hline & 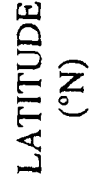 & 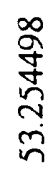 & 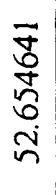 & 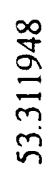 & 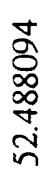 & $\begin{array}{l}\underset{\sigma}{\sigma} \\
\underset{\sim}{\sigma} \\
\frac{n}{n}\end{array}$ & $\begin{array}{l}8 \\
\text { లn } \\
\text { ஸे } \\
\text { ì } \\
\text { ñ }\end{array}$ & $\begin{array}{l}\bar{a} \\
\frac{\sigma}{0} \\
\underset{n}{n} \\
\text { n. }\end{array}$ & 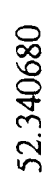 & \begin{tabular}{l}
$\bar{a}$ \\
$a$ \\
$\infty$ \\
$\curvearrowleft$ \\
\multirow{n}{n}{}
\end{tabular} & 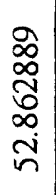 & 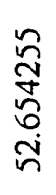 & 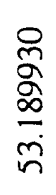 & $\frac{\hat{\frac{\pi}{n}}}{\frac{a}{n}}$ & 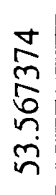 & 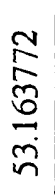 & 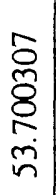 & 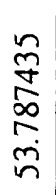 & 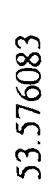 & 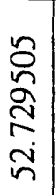 & 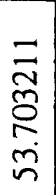 & 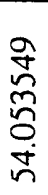 \\
\hline & $\approx$ & న్రి & $\mathbb{F}$ & $\stackrel{\Delta}{\sim}$ & $\underset{+}{\infty}$ & $\stackrel{\sim}{n}$ & ర్రి & $\underset{\sim}{\widetilde{J}}$ & \pm & $\frac{n}{n}$ & $\underset{\not}{0}$ & $\tilde{\sim}$ & 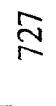 & $\underset{\sim}{\sim}$ & $\vec{\sim}$ & ָิ & $\stackrel{\infty}{=}$ & $\stackrel{ }{\equiv}$ & \& & $\stackrel{n}{\sigma}$ & $\stackrel{\infty}{\sim}$ & $\stackrel{\mathscr{M}}{\Xi}$ \\
\hline \multirow{3}{*}{$\begin{array}{l}\frac{1}{Z} \\
\frac{1}{3} \\
\frac{1}{0} \\
\frac{\sigma}{\alpha} \\
\frac{\alpha}{5}\end{array}$} & 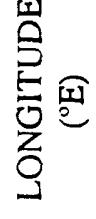 & 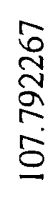 & 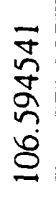 & 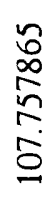 & 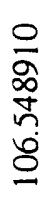 & 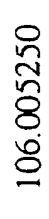 & 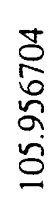 & 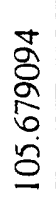 & 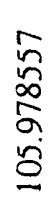 & 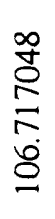 & 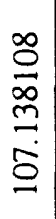 & 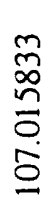 & 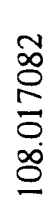 & 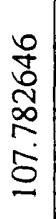 & 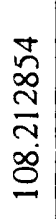 & 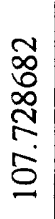 & 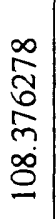 & $\begin{array}{l}n \\
\Sigma \\
\Sigma \\
n \\
o \\
0\end{array}$ & 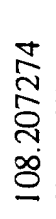 & $\begin{array}{l}8 \\
8 \\
0 \\
0 \\
\infty \\
8 \\
8 \\
0\end{array}$ & $\begin{array}{l}\stackrel{0}{o} \\
\stackrel{+}{a} \\
\bar{m} \\
\infty \\
o\end{array}$ & 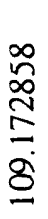 \\
\hline & 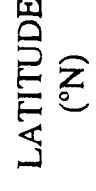 & $\begin{array}{l}8 \\
\delta \\
6 \\
80 \\
\dot{8} \\
\text { ஸ் }\end{array}$ & $\begin{array}{l}\frac{a}{d} \\
\frac{7}{0} \\
\dot{0} \\
\text { vi } \\
n\end{array}$ & 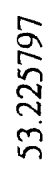 & 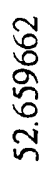 & $\begin{array}{l}\frac{n}{8} \\
\frac{8}{5} \\
\stackrel{n}{7}\end{array}$ & $\begin{array}{l}\underset{N}{N} \\
\infty \\
\infty \\
- \\
\end{array}$ & $\begin{array}{l}\frac{8}{0} \\
\frac{\overrightarrow{0}}{n} \\
\text { ñ }\end{array}$ & 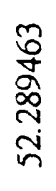 & \begin{tabular}{l}
$\frac{J}{\infty}$ \\
$\infty$ \\
$\infty$ \\
$\stackrel{0}{\circ}$ \\
\multirow{n}{n}{}
\end{tabular} & 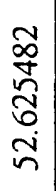 & 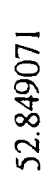 & 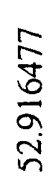 & $\begin{array}{l}\frac{a}{\tilde{n}} \\
\frac{n}{m} \\
\tilde{n}\end{array}$ & 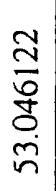 & 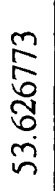 & 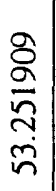 & $\begin{array}{l}8 \\
\mathscr{D} \\
\mathcal{N} \\
m \\
m \\
\tilde{n}\end{array}$ & 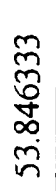 & $\begin{array}{l}\frac{\pi}{6} \\
\frac{\pi}{n} \\
\tilde{n} \\
n\end{array}$ & 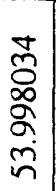 & 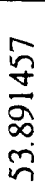 \\
\hline & $\hat{s}$ & - & $r$ & - & 6 & - & - & - & - & - & - & - & $m$ & $N$ & - & - & - & $\sim$ & - & - & -1 & - \\
\hline \multicolumn{2}{|c|}{ 罗宗 } & $\bar{\sim}$ & 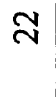 & $\stackrel{\sim}{\sim}$ & 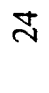 & $\approx$ & ㄴ & $\stackrel{\infty}{\sim}$ & 요 & กี & $\vec{m}$ & p & $\stackrel{\infty}{\text { ల }}$ & P & $\mathcal{F}$ & $\nabla$ & q & $\stackrel{\infty}{+}$ & 음 & กี & 芯 & 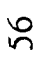 \\
\hline
\end{tabular}




\begin{tabular}{|c|c|c|c|c|c|c|c|c|c|}
\hline \multirow{5}{*}{ 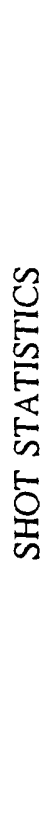 } & 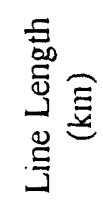 & 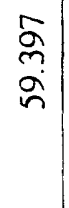 & $\begin{array}{l}\stackrel{a}{a} \\
\stackrel{N}{2}\end{array}$ & $\begin{array}{l}\mathscr{\infty} \\
\stackrel{+}{+} \\
\stackrel{f}{0}\end{array}$ & $\begin{array}{l}\text { f } \\
\text { d } \\
\stackrel{N}{N}\end{array}$ & 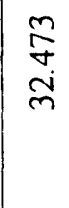 & $\underset{\sim}{\stackrel{\sim}{\sim}}$ & $\frac{\stackrel{2}{a}}{\stackrel{m}{m}}$ & $\begin{array}{l}\stackrel{0}{n} \\
+ \\
+\end{array}$ \\
\hline & 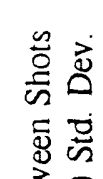 & $\stackrel{m}{m}$ & $\begin{array}{l}\dot{m} \\
\dot{m}\end{array}$ & $\underset{\infty}{\vec{\infty}}$ & $\stackrel{\infty}{\varrho}$ & i & 9 & $\bar{m}$ & $\ddot{\tilde{y}}$ \\
\hline & 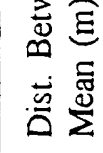 & $\stackrel{0}{\dot{n}}$ & $\begin{array}{l}\stackrel{8}{ } \\
\sim \\
\sim\end{array}$ & $\begin{array}{l}\stackrel{g}{g} \\
\stackrel{g}{q}\end{array}$ & $\stackrel{m}{\tilde{n}}$ & 占 & $\begin{array}{l}\bar{n} \\
\check{n}\end{array}$ & $\frac{n}{\circ}$ & $\stackrel{n}{\stackrel{n}{\sim}}$ \\
\hline & ă & $\stackrel{\widetilde{\sigma}}{=}$ & $\tilde{n}$ & $\frac{n}{\infty}$ & $\stackrel{2}{q}$ & డ్రి & $\stackrel{\infty}{ \pm}$ & $\tilde{\sigma}$ & $\vec{G}$ \\
\hline & $\hat{n}$ & $\stackrel{\infty}{\varrho}$ & $\stackrel{v}{n}$ & $\frac{n}{\infty}$ & $\stackrel{\tilde{\sigma}}{\sigma}$ & ర్రి & $\stackrel{\infty}{ \pm}$ & $\tilde{6}$ & $\bar{f}$ \\
\hline \multirow{3}{*}{ 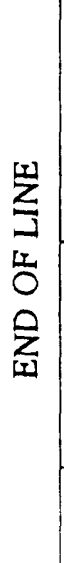 } & 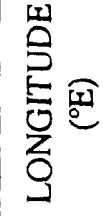 & 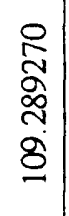 & 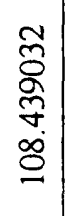 & 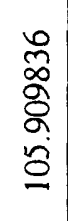 & $\begin{array}{l}\tilde{\hat{\infty}} \\
\tilde{\tilde{n}} \\
\dot{0} \\
\dot{0}\end{array}$ & $\frac{0}{2}$ & 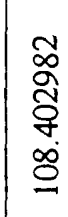 & $\begin{array}{l}\forall \\
0 \\
6 \\
6 \\
\infty \\
0\end{array}$ & 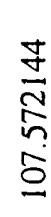 \\
\hline & 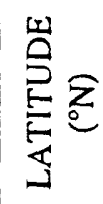 & \begin{tabular}{l}
\multirow{0}{0}{} \\
$\varnothing$ \\
$\alpha$ \\
$\hat{n}$ \\
$n$
\end{tabular} & 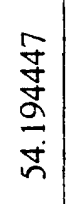 & 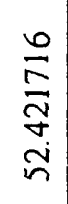 & $\begin{array}{c}\frac{\alpha}{\alpha} \\
\hat{n} \\
\frac{n}{n}\end{array}$ & 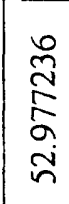 & 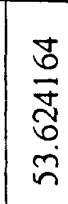 & 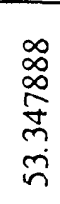 & 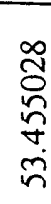 \\
\hline & on & $\stackrel{\infty}{=}$ & $\stackrel{v}{n}$ & 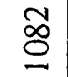 & $\stackrel{\alpha}{\sigma}$ & 8 & $\stackrel{\infty}{\square}$ & $\tilde{6}$ & $\bar{f}$ \\
\hline \multirow{3}{*}{ 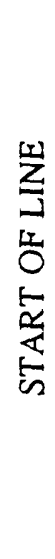 } & 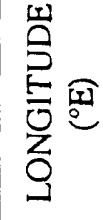 & 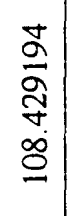 & 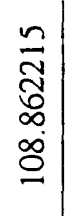 & 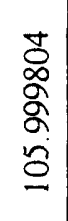 & 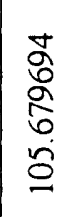 & $\begin{array}{l}8 \\
0 \\
0 \\
0 \\
0 \\
0\end{array}$ & 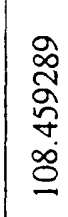 & 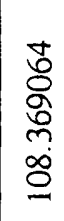 & 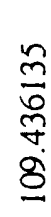 \\
\hline & 窝 & 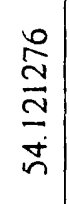 & $\begin{array}{l}\stackrel{8}{\circ} \\
\stackrel{0}{0} \\
\stackrel{5}{n}\end{array}$ & $\begin{array}{l}\hat{n} \\
o \\
0 \\
i \\
i \\
n\end{array}$ & $\begin{array}{l}0 \\
\hat{n} \\
\hat{\infty} \\
\infty \\
\vdots \\
\dot{n}\end{array}$ & $\begin{array}{l}\underset{D}{0} \\
\underset{\sigma}{N} \\
\underset{n}{n}\end{array}$ & 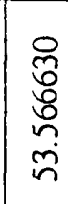 & $\begin{array}{l}\stackrel{0}{0} \\
0 \\
0 \\
\tilde{n} \\
\tilde{n}\end{array}$ & 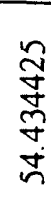 \\
\hline & के & - & - & 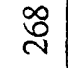 & - & $\underset{\sim}{\mathbb{f}}$ & - & - & - \\
\hline \multicolumn{2}{|c|}{ 呁。 } & $\infty$ & 8 & $\begin{array}{l}\overrightarrow{0} \\
\stackrel{0}{0}\end{array}$ & 总 & 章 & 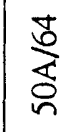 & 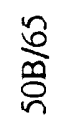 & $\stackrel{8}{\stackrel{8}{\vdots}}$ \\
\hline
\end{tabular}




\section{FIELD PROCEDURES}

\section{Navigation Logging}

Navigation data were taken from an Ashtech GPS Model XII Receiver, using frequency band L-1 (1575 MHz) and signal modulation code C/A. The Ashtech antenna was located on a cross tree above ship's bridge. Every 10 seconds, navigation information was transmitted through an RS-232 port to a personal computer for logging. Logged data consisted of date, time, latitude, longitude, ellipsoidal altitude, Horizontal Dilution of Precision (HDOP) value, speed, and heading. No raw GPS signal information was saved from the multichannel tracks. The personal computer also provided real time displays for use in navigating the ship, such as distance along line, distance off track, distance and time to next way point, etc. Archive media for the navigation data were floppy disks from the portable computer.

\section{Multichannel Shot Logging}

The multichannel seismic acquisition system consisted of a Texas Instruments DFS V computer with dual 1600-bpi tape drives. A Digital Timing Delay Generator, i.e., a calibrated digital clock, issued a master trigger to the DFS V which initiated the firing pulse to the air gun array. The desired shot interval of $50 \mathrm{~m}$ was approximated by matching the time between shots with averaged ship's speed. Shots were generally fired every 23 to 25 seconds corresponding to a ship's speed of 4.0 to $4.4 \mathrm{kts}$, although some lines had shot intervals as low as $22 \mathrm{~s}$ and as high as $27 \mathrm{~s}$. Firing rates were generally not varied within single lines.

Individual shot numbers were logged as the record or file number written onto the DFS $\mathrm{V}$ tape drive. Shot numbers for this cruise are therefore exactly equivalent to Field File Identification (FFID) numbers used in processing the multichannel data.

\section{Calibration of Navigation and Shot Numbers}

The shot numbers were calibrated to the navigation data manually at 15 -minute intervals. The navigation watch stander logged the DFS V file number as an entry in the hand-written navigation $\log$ at 15 -minute increments (about 35 to 40 shots). These entries were later typed as date-time-shot number files for processing the navigation, and are called shot-time calibration files.

\section{SOURCES AND MAGNITUDE OF NAVIGATION ERROR}

Three sources of error contribute to uncertainty in the Baikal navigation: (1) measurement limitation, (2) shot-time calibration, and (3) missing shots.

\section{Measurement Limitation}

This is error inherent in the hardware, software, and geometry of the GPS satellite and receiver system. The most variable source of error comes from the geometry of the satellites used in calculating the navigation position, and is referred to as the Dilution of Precision (DOP) factor (Milliken and Zoller, 1980; Hurn, 1989). DOP's are numerical values that can be recorded for 3-dimensional solutions (PDOP - Position Dilution of Precision), for 2-dimensional solutions (HDOP - Horizontal Dilution of Precision or VDOP - Vertical Dilution of Precision), and for time solutions (TDOP - Time Dilution of Precision). For the Baikal data, HDOP was recorded 
rather than PDOP because navigation solutions on a lake surface are essentially made at the same vertical elevation. This also allowed for 3-satellite solutions to be used if 4-satellite solutions were not available. HDOP values for good satellite geometries are generally between 1 and 4; values for poor geometry are much larger.

(Table 2):

Other sources of measurement error are generally constant and are summarized below

TABLE 2: Typical Sources of Measurement Error ${ }^{1}$

\begin{tabular}{|c|c|c|}
\hline SOURCE & ERROR (M) & DESCRIPTION \\
\hline Satellite Clock & .6 & Clock Drift \\
\hline Ephemeris & .6 & Uncertainty in satellite orbit \\
\hline Receiver & 1.2 & Clock synchronization with satellite \\
\hline Atmospheric/Ionospheric Effect & 3.7 & Signal propagation delays \\
\hline Selective Availability ${ }^{2}$ & $0-7.6$ & Random signal degradation \\
\hline Root-Square Sum ${ }^{3}$ & $4-9$ & Estimated Combined Error \\
\hline Baikal estimate & 7 & Assumed mid-value \\
\hline
\end{tabular}

${ }^{1}$ Source: Hurn (1989).

${ }^{2}$ Selective Availability is unannounced degradation of the signal by Department of Defense. This is the most unconstrained source of error. $7.6 \mathrm{~m}$ is considered worst case.

${ }^{3}$ Root-Square Sum is the square root of the sum of the squares.

Total measurement error is calculated by taking the product of the HDOP value with the root-square sum of the other sources of error (Hurn, 1989). For the Baikal data, a representative root-square sum value of $7 \mathrm{~m}$ is assumed. The measurment error in meters is therefore taken as 7 times HDOP.

\section{Shot-Time Calibration}

This refers to the logging of time and/or shot numbers at the 15 -minute calibration points. The magnitude of this error is related to human error in logging time and and/or shot number correctly at the calibration points. Ideally, the clock used to fire the airgun array should have been synchronized to the GPS clock and shot-time recorded automatically to a fractional second. In actuality, the (GPS) times at calibration points were logged to whole seconds (best case) or whole minutes (worst case). Logging time in whole minutes was most common at the beginning of the cruise because of language difficulties between some of the Russian and American watch standers. Very few of these human errors occurred in the latter part of the cruise.

These errors in time are given values of $1 \mathrm{~s}$ (for whole second logging) and $30 \mathrm{~s}$ (for whole minute logging, assuming time is rounded to the nearest whole minute). To convert these errors in time to position error in meters, the mean distance between 10-s positions was either divided by 10 (to get mean distance for 1-s interval) or multiplied by 3 (to get mean distance for 30 -s interval). The magnitude of the uncertainty due to the 1 -s error is about $2 \mathrm{~m}$; that for the 30 -s case is about $70 \mathrm{~m}$. 
Shot-time errors could also occur if shot numbers were improperly logged. Occassionally, shots from the navigation log showed gross inconsistency with the multichannel observer's logs, indicating that the watch stander erroneously recorded the shot/file number. The more obvious errors could be corrected after comparison of the navigation logs with the multichannel observer's logs.

\section{Missed Shots}

This refers to situations in the field in which the airgun array continued firing at even increments of time even though the DFS $\mathrm{V}$ was not recording the shot or incrementing the file/shot number. The actual shot-time calibration points are based on the field file numbers from the DFS-V, rather than the actual firing of the airgun array, which means missed shots are not automatically counted. In the first half of the cruise, several poor quality tapes together with excessive parity errors on one of the tape drives resulted in missed shots. In the second half of the cruise, when only one tape drive was used, two firings were missed at every tape change because of the physical limitations in removing and reloading a single tape drive. For times in which missed shots occured, the navigation interpolation must include a correction for the additional shots that occurred in that interval.

In order to compensate for the missed shots, the concept of a "pop" number is introduced for the processing. The pop number differs from the shot number in that the pop increments every time the air-gun array fires whereas the shot number increments only when the DFS V writes a file to tape. Missed shots (i.e., those not recorded on the DFS V) will introduce a systematic offset between the pop number and the shot number. Both pop and shot numbers should be identical for lines in which no recording errors (i.e., missed shots) occur. The concept of the pop number for the Baikal data is used only in the processing of the navigation information and is not logged in any of the final archive navigation files.

Figure 2 shows a hypothetical situation to emphasize the effects of poor logging and missed shots on estimating the time of each shot. Time is shown incrementing to the right, with a 15-minute calibration interval noted. (Assuming a constant ship's speed of $4.5 \mathrm{kts}$, this hypothetical time curve can also be equated with distance, or 15 minutes equals about $2.1 \mathrm{~km}$ ). Suppose there are 15 shot numbers recorded from minute 1 to minute 15 (i.e., 15 files written on the DFS V). If there are no missing shots, then the shot number equals the pop number and configuration A holds for interpolating 15 even intervals for the shot times. However, if there are 5 missed shots (shown as $*$ ), then the pop number exceeds the shot number by 5 and configuration B ( 20 even time intervals) is the accurate representation for determining the shot times. If the missed shots are not accurately accounted for, then the shot time will be correct at the calibration points (shots 0 and 15), but will be misestimated (by up to 2.25 minutes for shot 6). This timing error for shot 6 equals a distance mislocation of $313 \mathrm{~m}$ in this example. In general, this error is greatest at the position of the missing shots and decreases toward the 15minute calibration points.

If the time is not logged properly for the 15-minute calibration, then a shot-time calibration error is introduced. For whole minute logging, the timing error is assumed to be 30 s (i.e., watch stander recorded the whole minute to the nearest whole minute). The effect on estimating shot time is shown by configuration $\mathrm{C}$ in Figure 2, where one scenario is illustrated in which the whole minutes reported are considered to be off by 30 seconds at each calibration 


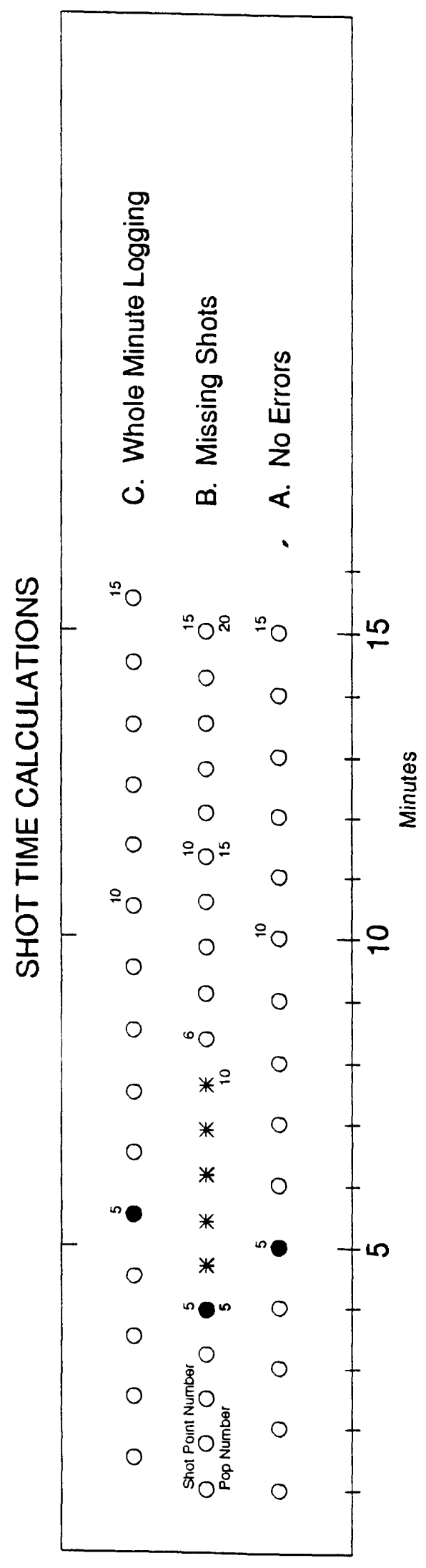

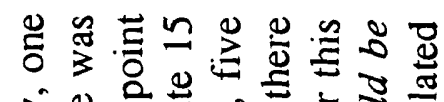

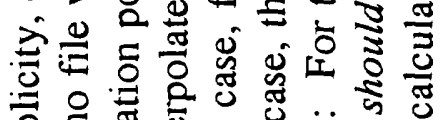
क्व

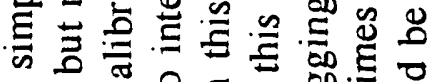

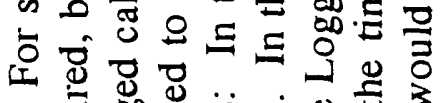

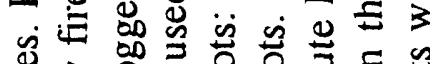

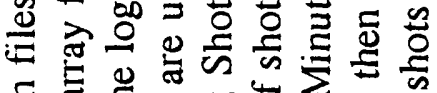

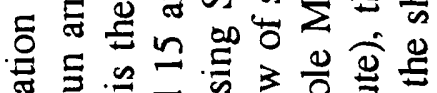

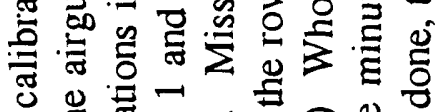

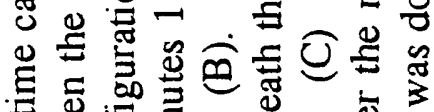

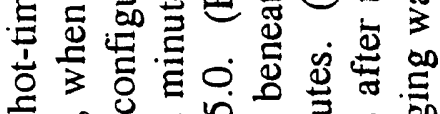

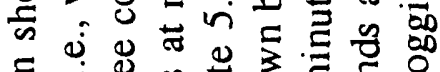

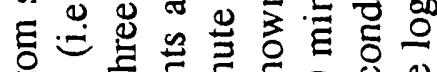

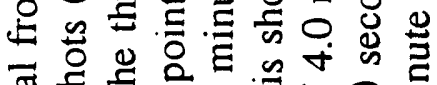
ส 份部

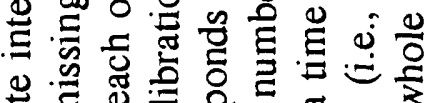

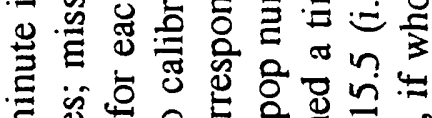
हี

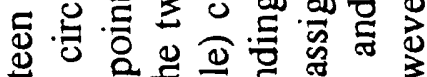

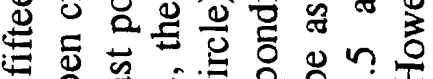

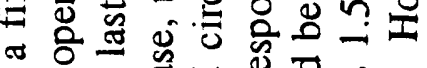
ఏ즈웛

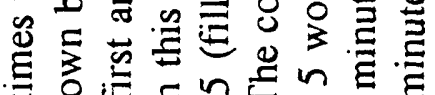

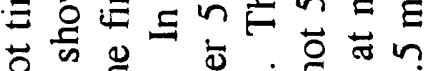

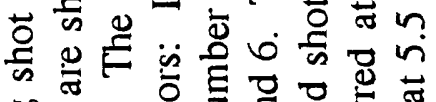

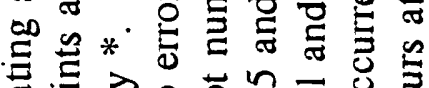

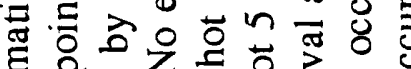
ह 중

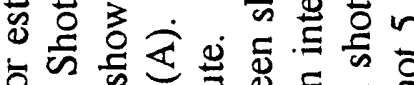

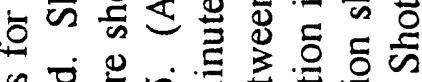

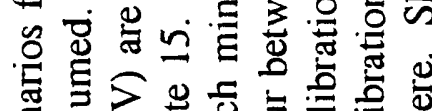

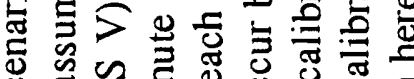

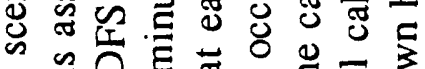

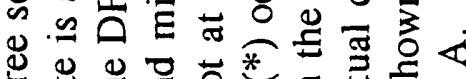

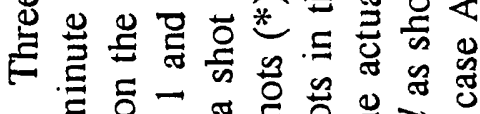
人 ह

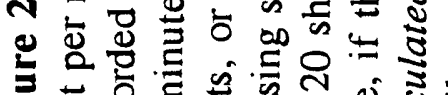

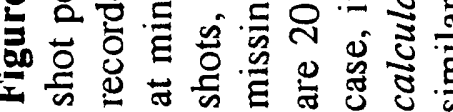


point. Configuration $\mathrm{C}$ shows what the actual shot times should have been; configuration $\mathrm{A}$ shows how they would be calculated in the absence of the seconds information. In this case, the calibration shots 0 and 15 would have an error of 30 seconds $(69 \mathrm{~m})$, as would all shots in that interval.

The missing-shot error is actually treated as a correction to the data rather than as an error; i.e., missing shots are accounted for in the processing, but no missing-shot error is calculated or assigned. This assumes that the missing shots were reasonably carefully logged and that any unlogged shots are not enough to bias an entire line. Most of the missing shots compensated in the processing are single or double shots (representing a timing correction of up to $45 \mathrm{~s}$ at the position of the missed shots, or up to about 100 -m correction). The most missing shots that occurred at one interval are 17 on line 6 . One or two missed shots generally has no visible effect on fold coverage of the multichannel data. However, the 17-shot gap in line 6 was sufficient to cause a small data gap at SP 820-821.

\section{PROCESSING STRATEGY}

Two basic inputs are used in processing the navigation: the raw 10-s locations and listings of the shot-time calibrations points. The steps in processing the navigation data (Figure 3) were:

(1) Inspect the overall quality of the raw navigation data by looking at gaps and calculating the distance between adjacent fixes for each 10-second recording point. Plot ellipsoidal height and HDOP value for general quality control.

(2) Evaluate the quality of the shot-time logging, which was done by calculating firing rate for each 15-minute calibration interval and inspecting the scatter. Ideally, with a constant firing rate of the airgun array, the recomputed firing times for each 15 -minute period should also be constant, or vary slightly when the firing rate was infrequently adjusted for changes in the ship's speed.

(3) Edit the calibration files for obvious typographical errors based on the magnitude of the scatter observed in plots from step 2.

(4) Develop and merge the best estimates of the number and positions of missing shots with the calibration files. In this step, the pop numbers were defined.

(5) Generate final latitude/longitude positions by using the updated calibration files to interpolate times for each pop, which are then merged with the raw navigation files to interpolate latitude and longitude for the pop times. Final archive navigation drops the pop numbers which do not have accompanying shot numbers defined.

(6) Inspect the quality of the shot navigation by looking at the distance between adjacent pops for each line.

(7) Assign an error to each line based on the quality of the raw navigation and the errors introduced in the calibration files.

Plots showing the results of step 1 are given in Appendix 1 for each line. Plots resulting from steps 2, 4, 5, and 6, are given in Appendix 2 for each line. 

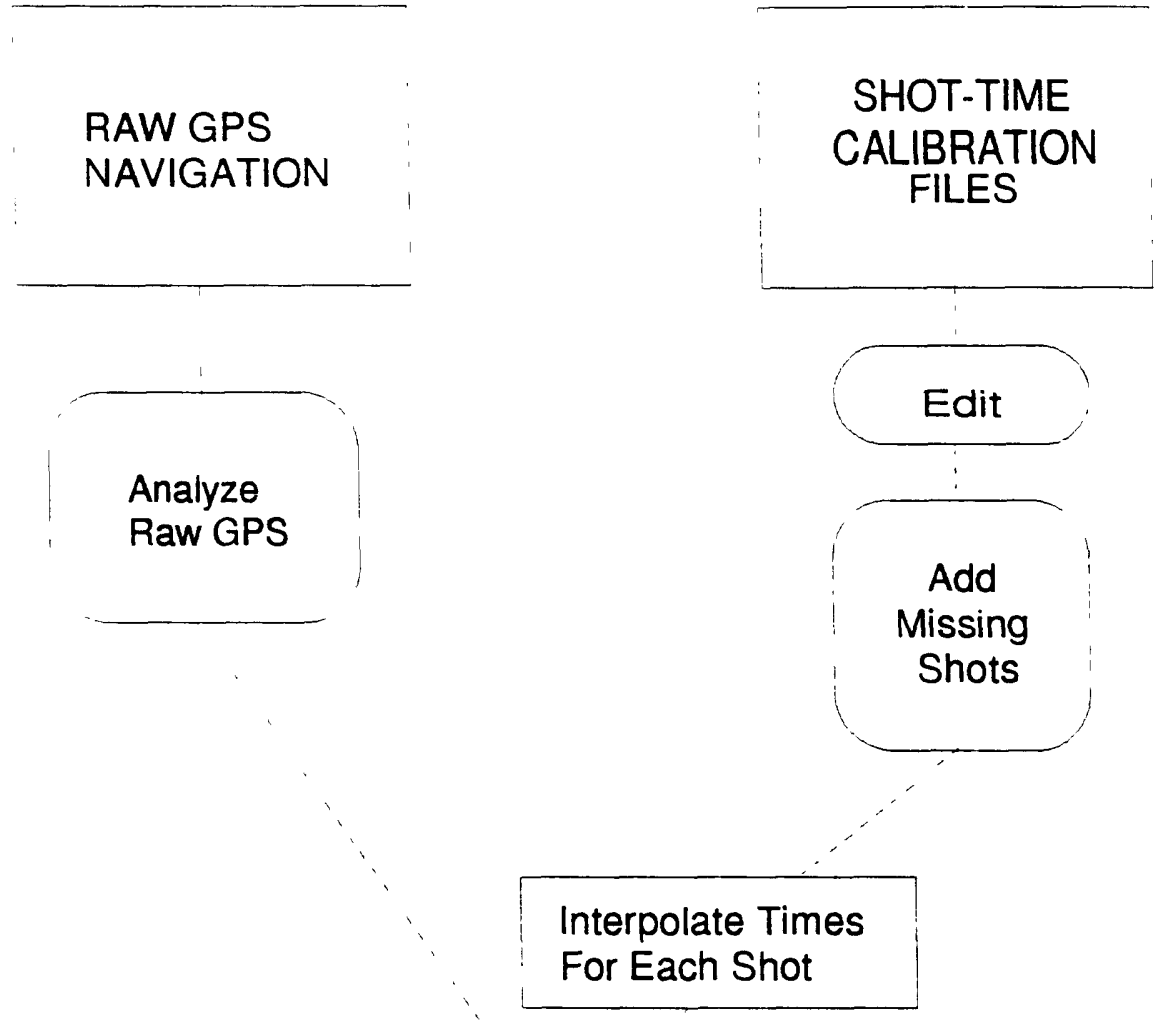

Interpolate Position For Each Shot

Analyze

Raw GPS

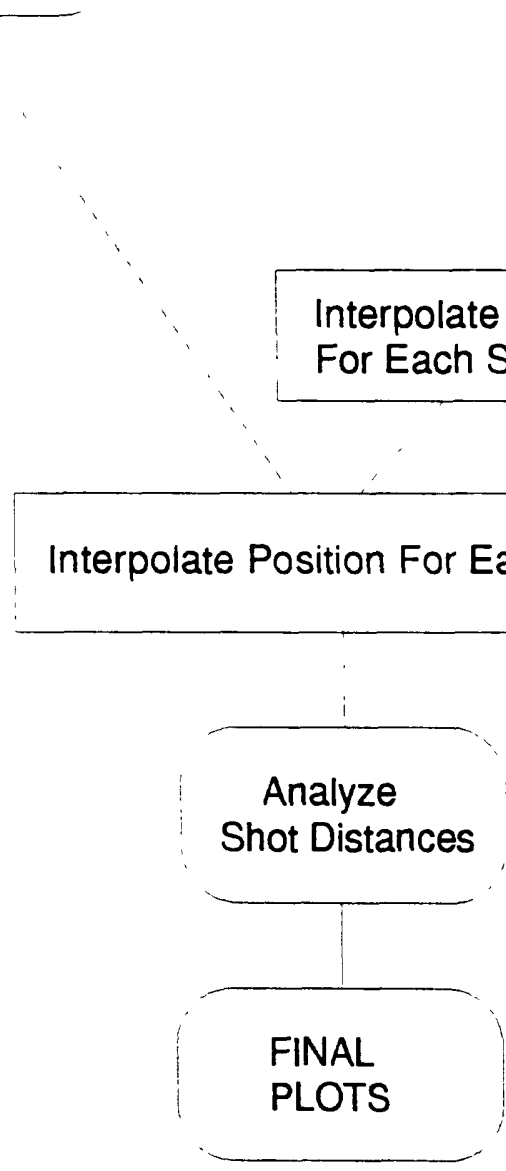

Figure 3. Flow chart of the navigation processing. 
TABLE 3: DATA GAPS

\begin{tabular}{|c|c|c|c|c|c|c|c|}
\hline \multirow{2}{*}{ Line No. } & \multicolumn{2}{|c|}{ START OF GAP } & \multicolumn{2}{|c|}{ END OF GAP } & \multirow{2}{*}{$\begin{array}{c}\text { LENGTH } \\
(\mathrm{s})\end{array}$} & \multicolumn{2}{|c|}{ SHOTS IN GAP } \\
\hline & Date & Time & Date & Time & & Total & SP Nos. \\
\hline 6 & 920826 & 175622 & 920826 & 180302 & 400 & 16 & $909-924$ \\
\hline 7 & 920829 & 190902 & 920829 & 191012 & 70 & 2 & $526-527$ \\
\hline 7 & 920829 & 191032 & 920829 & 191302 & 150 & 6 & $529-534$ \\
\hline 8 & 920826 & 233412 & 920826 & 233522 & 70 & 2 & $284-285$ \\
\hline 8 & 920827 & 011652 & 920827 & 012612 & 560 & 23 & $530-552$ \\
\hline 8 & 920827 & 012702 & 920827 & 013242 & 340 & 14 & $555-568$ \\
\hline 8 & 920827 & 024802 & 920827 & 025102 & 180 & 7 & $748-754$ \\
\hline 11 & 920922 & 195412 & 920922 & 195632 & 140 & 6 & $324-329$ \\
\hline 13 & 920920 & 225812 & 920920 & 230212 & 240 & 10 & $444-453$ \\
\hline 13 & 920920 & 234632 & 920920 & 235302 & 390 & 15 & $555-569$ \\
\hline 13 & 920921 & 035032 & 920921 & 035322 & 170 & 7 & $1107-1113$ \\
\hline 13 & 920921 & 035452 & 920921 & 035722 & 150 & 6 & $1117-1122$ \\
\hline 13 & 920921 & 065652 & 920921 & 070112 & 260 & 11 & $1543-1553$ \\
\hline 13 & 920921 & 121612 & 920921 & 121822 & 130 & 6 & 2353-2358 \\
\hline 17 & 920916 & 050002 & 920916 & 054122 & 2480 & 108 & 2866-2973 \\
\hline 26 & 920831 & 174122 & 920831 & 175132 & 610 & 25 & $148-172$ \\
\hline 34 & 920922 & 003132 & 920922 & 003432 & 180 & 6 & $509-514$ \\
\hline 38 & 920910 & 134342 & 920910 & 134642 & 180 & 7 & $700-706$ \\
\hline 46 & 920913 & 082032 & 920913 & 082432 & 240 & 10 & $22-31$ \\
\hline 46 & 920913 & 083642 & 920913 & 083802 & 80 & 3 & $63-65$ \\
\hline 50 & 920912 & 181132 & 920912 & 181232 & 60 & 2 & $236-237$ \\
\hline 50 & 920912 & 193252 & 920912 & 193352 & 60 & 2 & $432-433$ \\
\hline 52 & 920922 & 090112 & 920922 & 090232 & 80 & 4 & $58-61$ \\
\hline 66 & 920916 & 164852 & 920916 & 170832 & 1180 & 9 & $51-59$ \\
\hline 66 & 920917 & 112842 & 920917 & 113242 & 240 & 2 & $605-606$ \\
\hline
\end{tabular}




\section{RESULTS}

\section{Quality of the Raw Navigation}

Raw navigation information is illustrated in Appendix 1 with four plots for each line: distance between each data point (top plot), time between each data point (second plot), HDOP value associated with each data point (third plot), and ellipsoidal height (bottom plot).

The plots of distance between each point show considerable scatter on some lines (e.g., line 4 , with a standard deviation of $13.6 \mathrm{~m}$ ) and remarkably little scatter on others (lines 23 , with a standard deviation of $1.44 \mathrm{~m}$ ). The 10 -s positions average about $20-23 \mathrm{~m}$ apart throughout the cruise, and the standard deviations range from about $5 \%$ (line 23) to more than $50 \%$ (line 4).

In general, the scatter is correlated to changes in HDOP value (e.g., HDOP changes rapidly on line 4 and is less variable for line 23). This correlation suggests that the scatter is best explained by changes in satellites and/or satellite geometry used in computing successive positions along a track line.

Because data points were logged every ten seconds, the plot of time between data points should be constant at $10 \mathrm{~s}$. Deviations from this represent data gaps. Note that data gaps in time are duplicated as large peaks in distance between points (e.g., line 8 at $3.75-4.0$ hours). Table 3 summarizes the data gaps for the 14 lines with gaps of 1 minute or more (i.e., 6 or more data points lost). The two largest gaps were on lines 17 and 17A, in which data losses occurred of 41.3 and 19.6 minutes respectively.

Measurement error can be estimated directly from the raw navigation. Table 4 lists the mean measurement error for each line. As defined earlier, the measurment error is given as 7 $x$ HDOP, where HDOP is the mean HDOP calculated for each line. These errors range from $7.49 \mathrm{~m}$ (lines 42 and 52) to $22.75 \mathrm{~m}$ (line 40 ). Figure 4 (top plot) illustrates these errors, shown as X's together with estimates of the scatter (standard deviation of the calculated distance between data points), shown as O's. In general, the two values are in good agreement. The error bars are the standard deviation of the error, and are primarily an indicator of the amount of scatter in the HDOP value.

\section{Quality of the Calibration Files}

Plots of the initial and final calibration files are shown as the upper two plots in the figures in Appendix 2. The calibration curves are displayed as firing rates, calculated by dividing the time between control points by the number of shots between control points. The control points plotted in the edited (final) version use pops between control points to calculate the shot interval, although the information is plotted against shot numbers. Ideally, these lines should be constant and horizontal along the line, because the pops were fired by time, or they should show a small offset where the shot number may have been adjusted for small changes in the speed of the ship.

All of the plots of the initial calibration files show a boxy pattern, in which the shot interval varies between high $(24-27 \mathrm{~s})$ and low $(21-23 \mathrm{~s})$ values. This is a direct result of poor record keeping and missing shots. After editing, some of the lines have excellent (i.e., flat) 
curves (e.g., lines 54 and 58). Many of the lines show considerable improvement in the flatness of the calibration curves after editing (e.g., lines 11 and 13) whereas a few show little or no improvement (e.g., line 15). The final calibration curves have been assigned a rating of 1 (time logged to whole seconds) or 30 (time logged to whole minutes); these values are listed in Table 4 , together with the calculated error in distance that these timing errors correspond to.

Lines in which some or all of the calibration points were logged to whole minutes were given ratings of 30 , even if the final curves looked reasonable, because of the absolute uncertainty in shot time based on the poor time-keeping, as described with Figure 2 . For example, line 6 is given a 30-s error value for the entire line even though whole minute logging occurred only in the first half of the line. Line 7 , one of the long tie lines across the Selenga Delta, contains whole-minute logging for only about $25 \%$ of the line (600 shots from about SP $1000-1600$ ), but is also assigned a 30-s error for the whole line. The portions of lines which had whole-minute logging are clearly marked in Appendix 2, and should be inspected to clarify how much of any line is affected by the whole-minute logging.

The shot-time calibration error was combined with the measurement error using a rootsquare sum to yield total error for each line. Figure 4B shows the total error (i.e., for both measurement and shot-calibration errors). A comparison of this figure with the measurement error (Figure 4A) reveals the large contribution of the shot-time calibration error due to the whole-minute logging (30-s error). For example, line 4, which has a measurement error of 8.89 $\mathrm{m}$, has a combined total error of about $74 \mathrm{~m}$ when the shot-time calibration is added. The difference between the measurement error and the total error for lines with a 1-s shot-time calibration error is generally negligible.

\section{Quality of the Distance Between Shots}

The quality of both the raw navigation and the calibration files affects the calculations of distances between shots. These distances are shown in two forms in Appendix 2 (lower two plots): as relative distance between shots along each line (third plot down) and a histogram of distances (lowest plot). The plot of distance between shots gives a good representation of the magnitude of the scatter along the line; the histogram illustrates the spread of the scatter. All plots are at the same scale for easy comparison. The standard deviations given on the plots are based on the relative distances between shots, rather than the uncertainty associated with the absolute position of the shot.

The scatter recorded for distances between shots essentially mirrors that of the raw navigation plots (compare plots for each line in Appendix 1 and Appendix 2). The distances between shots are important for evaluating the assumption of $50-\mathrm{m}$ shots used to process the multichannel data. Figure 5 shows the mean distance between shots (X) together with the standard deviation (error bar) for each line. The ideal shot distance of $50 \mathrm{~m}$ is also shown as a horizontal line.

Except for line 30, the error bars encompass the 50-m ideal shot spacing. Hence the assumption of 50-m shots is valid for processing the data. Line 30 has a mean shot spacing of $57-\mathrm{m}$, but is a very short line (174 shots) and therefore is a very small element of the entire data set. 


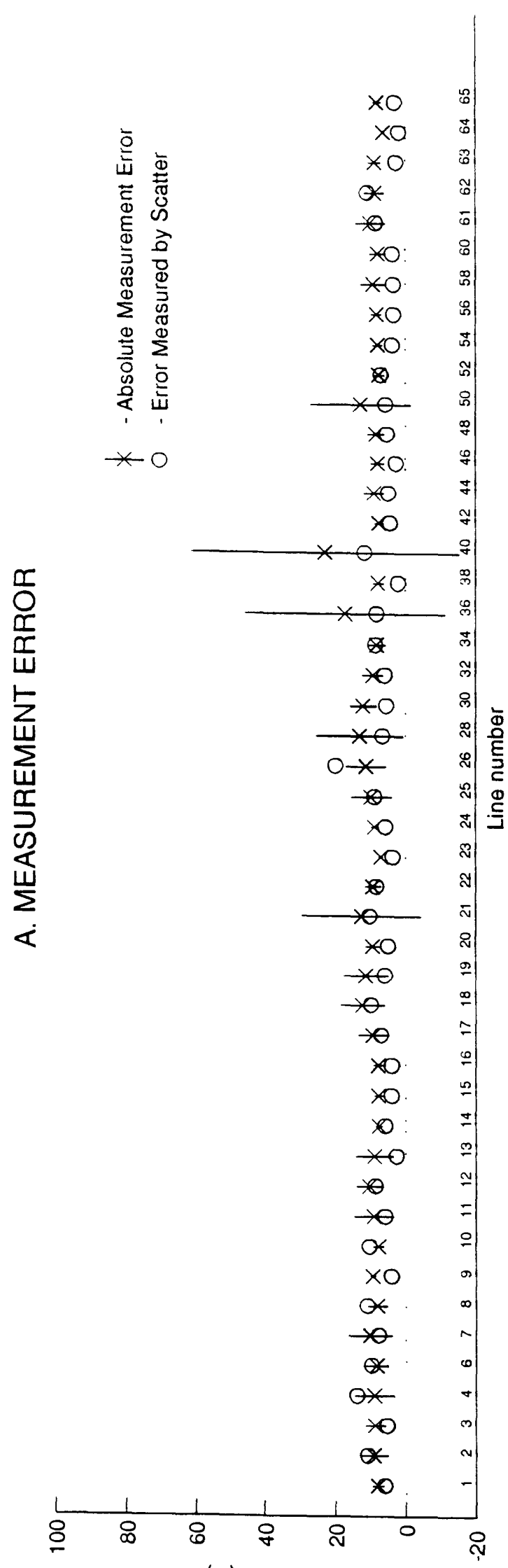

(w) eouels!o

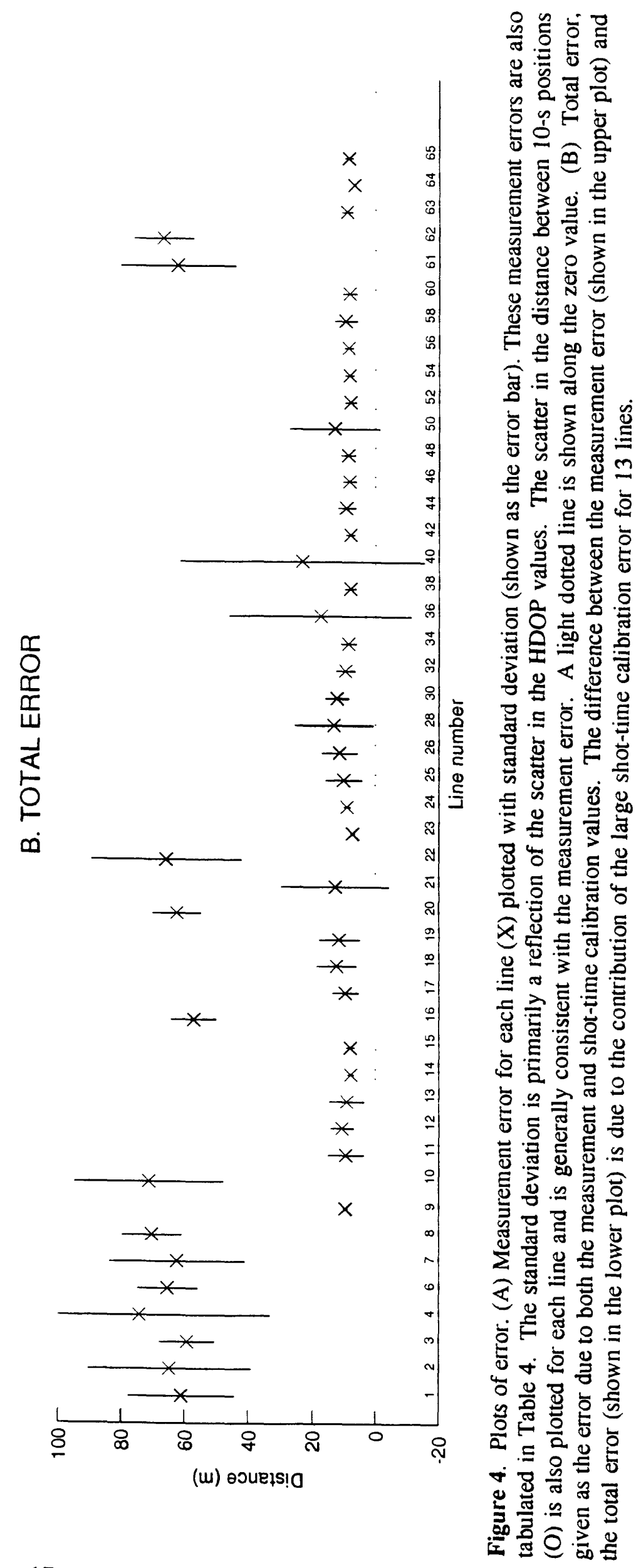


TABLE 4: NAVIGATION ERROR AND DATA QUALITY

\begin{tabular}{|c|c|c|c|c|c|c|}
\hline \multirow{2}{*}{$\begin{array}{c}\text { LINE } \\
\text { NUMBER }\end{array}$} & \multirow{2}{*}{$\begin{array}{l}\text { MEAN SHOT } \\
\text { DISTANCE }\end{array}$} & \multirow{2}{*}{$\begin{array}{c}\text { MEASURE- } \\
\text { MENT } \\
\text { ERROR }^{2} \\
\end{array}$} & \multicolumn{2}{|c|}{ SHOT-TIME CALIB. } & \multirow{2}{*}{$\begin{array}{l}\text { TOTAL } \\
\text { ERROR }^{5}\end{array}$} & \multirow[t]{2}{*}{ RATING $^{6}$} \\
\hline & & & Factor $^{3}$ & Error $^{4}$ & & \\
\hline 1 & 52.84 & 7.98 & 30 & 60.51 & 61.03 & Poor \\
\hline 2 & 54.47 & 9.17 & 30 & 64.14 & 64.79 & Poor \\
\hline 3 & 49.47 & 8.68 & 30 & 58.50 & 59.14 & Poor \\
\hline 4 & 54.83 & 8.89 & 30 & 73.68 & 74.21 & Poor \\
\hline 6 & 50.59 & 8.33 & 30 & 64.83 & 65.36 & Poor \\
\hline 7 & 50.74 & 10.15 & 30 & 61.50 & 62.33 & Poor \\
\hline 8 & 53.02 & 8.05 & 30 & 69.84 & 70.30 & Poor \\
\hline 9 & 49.95 & 9.38 & 1 & 2.01 & 9.59 & Excellent \\
\hline 10 & 55.33 & 7.70 & 30 & 70.71 & 71.13 & Poor \\
\hline 11 & 50.03 & 9.10 & 1 & 2.23 & 9.37 & Excellent \\
\hline 12 & 47.86 & 10.43 & 1 & 1.99 & 10.62 & Excellent \\
\hline 13 & 51.71 & 8.89 & 1 & 2.15 & 9.15 & Excellent \\
\hline 14 & 45.37 & 7.63 & 1 & 1.85 & 7.85 & Excellent \\
\hline 15 & 51.06 & 7.77 & 1 & 2.20 & 8.07 & Excellent \\
\hline 16 & 46.74 & 7.84 & 30 & 56.40 & 56.94 & Poor \\
\hline 17 & 49.22 & 9.31 & 1 & 2.28 & 9.58 & Excellent \\
\hline 18 & 49.45 & 12.25 & 1 & 2.02 & 12.41 & Good \\
\hline 19 & 52.21 & 11.27 & 1 & 2.27 & 11.50 & Excellent \\
\hline 20 & 50.76 & 9.31 & 30 & 61.53 & 62.23 & Poor \\
\hline 21 & 55.93 & 12.46 & 1 & 2.37 & 12.68 & Good \\
\hline 22 & 52.49 & 9.31 & 30 & 64.89 & 65.55 & Poor \\
\hline 23 & 52.21 & 6.93 & 1 & 2.09 & 7.24 & Excellent \\
\hline 24 & 50.87 & 8.68 & 1 & 2.04 & 8.92 & Excellent \\
\hline 25 & 51.57 & 9.80 & 1 & 2.27 & 10.06 & Excellent \\
\hline 26 & 53.26 & 11.13 & 1 & 2.31 & 11.37 & Excellent \\
\hline 28 & 47.06 & 12.95 & 1 & 1.93 & 13.09 & Good \\
\hline 30 & 57.32 & 12.04 & 1 & 2.22 & 12.24 & Good \\
\hline 32 & 51.35 & 9.17 & 1 & 2.19 & 9.43 & Excellent \\
\hline 34 & 50.98 & 8.12 & 1 & 2.33 & 8.45 & Excellent \\
\hline
\end{tabular}




\begin{tabular}{|c|c|c|c|c|c||c||}
\hline 36 & 53.18 & 17.08 & 1 & 2.39 & 17.25 & Good \\
\hline 38 & 50.97 & 7.70 & 1 & 2.06 & 7.97 & Excellent \\
\hline 40 & 56.18 & 22.75 & 1 & 2.28 & 22.86 & Good \\
\hline 42 & 52.74 & 7.49 & 1 & 2.22 & 7.81 & Excellent \\
\hline 44 & 51.08 & 8.96 & 1 & 2.08 & 9.20 & Excellent \\
\hline 46 & 51.93 & 7.84 & 1 & 2.20 & 8.14 & Excellent \\
\hline 48 & 54.45 & 8.33 & 1 & 2.21 & 8.62 & Excellent \\
\hline 50 & 54.27 & 12.67 & 1 & 2.22 & 12.86 & Good \\
\hline 52 & 50.81 & 7.49 & 1 & 2.19 & 7.80 & Excellent \\
\hline 54 & 50.49 & 7.84 & 1 & 2.20 & 8.14 & Excellent \\
\hline 56 & 50.16 & 8.19 & 1 & 2.18 & 8.47 & Excellent \\
\hline 58 & 51.16 & 9.17 & 1 & 2.14 & 9.42 & Excellent \\
\hline 60 & 52.66 & 7.84 & 1 & 2.22 & 8.15 & Excellent \\
\hline $61 / 10 \mathrm{~A}$ & 49.49 & 10.01 & 30 & 60.90 & 61.72 & Poor \\
\hline $62 / 3 \mathrm{~A}$ & 53.33 & 8.75 & 30 & 65.52 & 66.10 & Poor \\
\hline $63 / 40 \mathrm{~A}$ & 52.46 & 8.68 & 1 & 2.20 & 8.95 & Excellent \\
\hline $64 / 50 \mathrm{~A}$ & 50.51 & 6.37 & 1 & 2.02 & 6.68 & Excellent \\
\hline $65 / 50 \mathrm{~B}$ & 50.15 & 8.12 & 1 & 2.17 & 8.40 & Excellent \\
\hline $66 / 17 \mathrm{~A}$ & 257.15 & 8.61 & 1 & 2.19 & 8.88 & Excellent \\
\hline
\end{tabular}

${ }^{1}$ Mean Shot Distance - Mean distance between successive firings of the airgun array after taking into account all missing shots. This same number is given in Appendix 2 on the top plot for each line.

${ }^{2}$ Measurment Error - Error due to inherent limitations of the satellite-receiver system. This number is taken to be 7 times mean HDOP, as discussed in the text.

${ }^{3}$ Shot-Time Calibration Factor - This is the factor assigned to each line based on the quality of the shot-time calibrations. 1 refers to times recorded to whole seconds; 30 is for times recorded to nearest whole minute.

${ }^{4}$ Shot-Time Calibration Error - This error is given as the average distance between 10-s navigation locations (top plot of Appendix 1) multiplied by .1 (1-s factor) or 3 (30-s factor).

${ }^{5}$ Total Error - Taken as the root-square sum of the measurement and shot-calibration errors.

${ }^{6}$ Rating - Excellent is for error of 6-12 m; Good is for errors of $12-24 \mathrm{~m}$; Poor is for errors $>50 \mathrm{~m}$. 

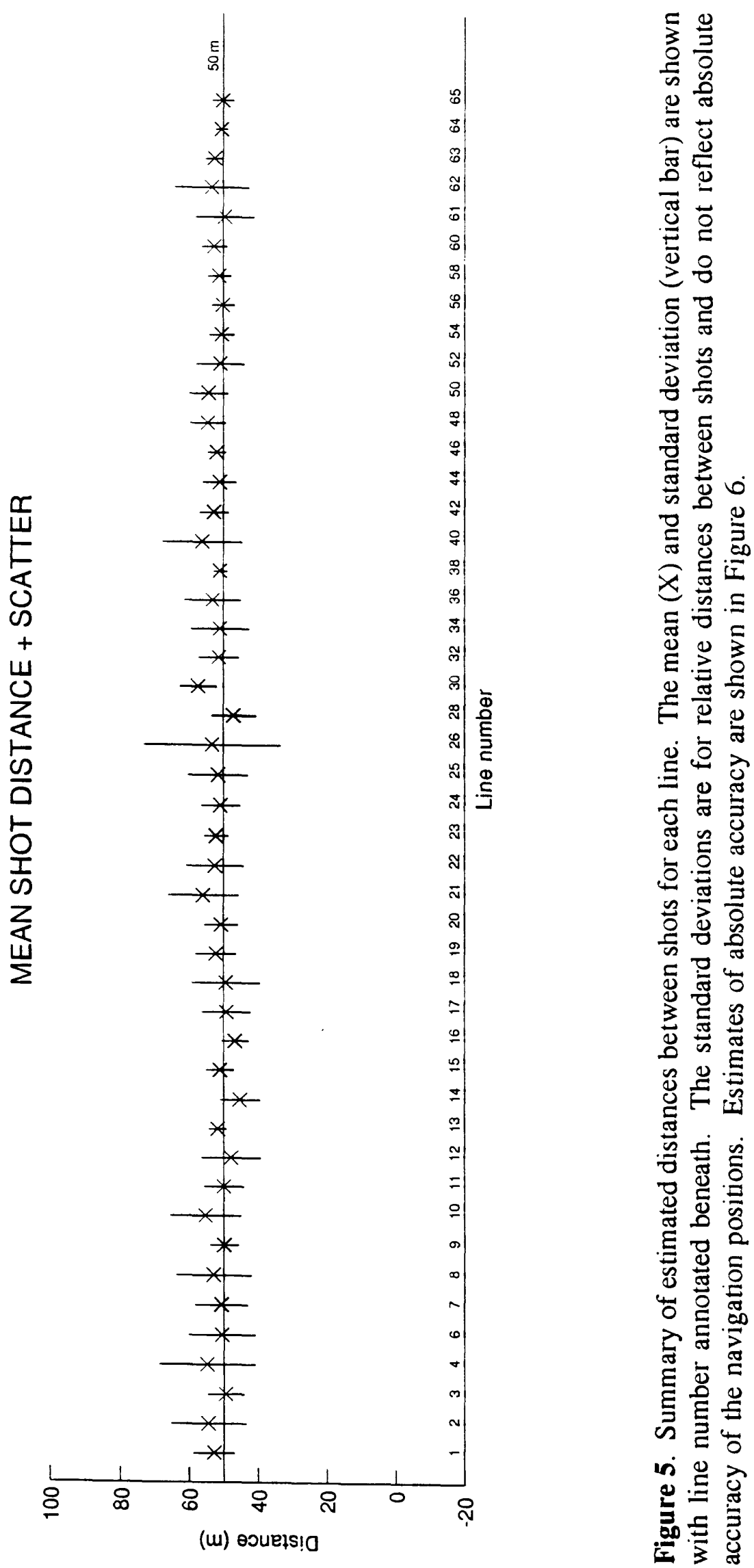


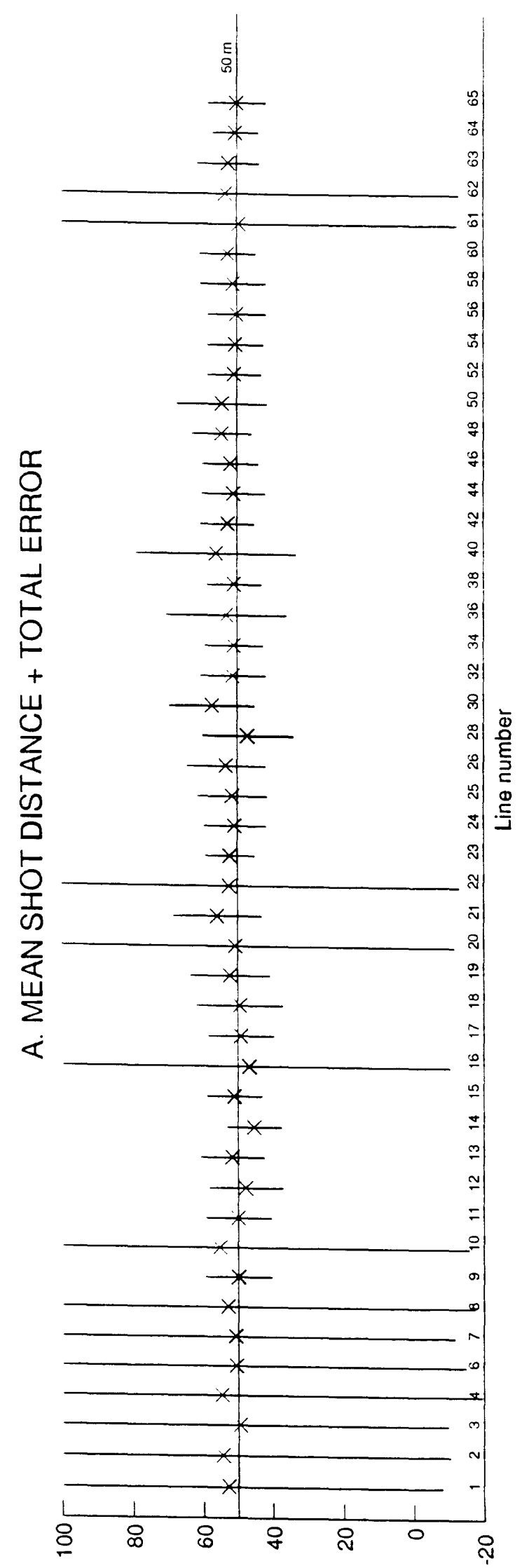

(w) eวuels!a

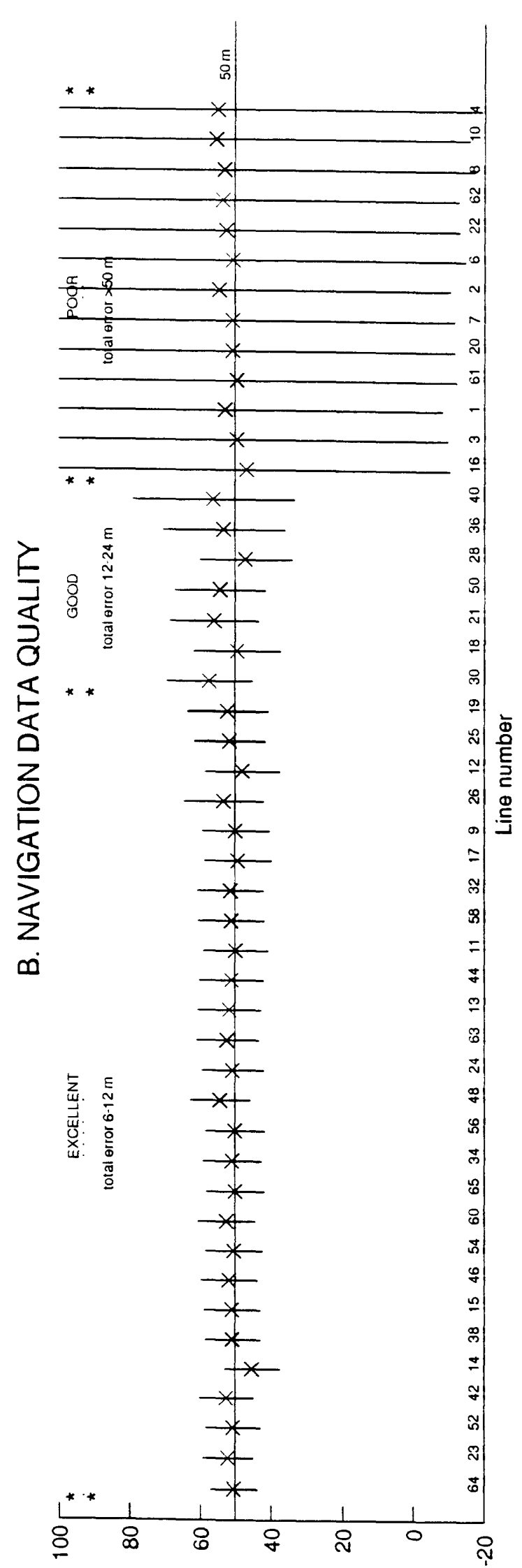

(w) exuels!a 


\section{Quality of Shot Locations}

For the purposes of this report, an error estimate is assigned to each line based on the mean characteristics of the line (e.g., mean HDOP), rather than assigning an error estimate to each individual shot. This gives a general guide for interpreting the navigation quality for each multichannel profile.

Figure 6A gives the total position uncertainty for each line plotted as an error bar around the mean shot distance. The value of the error bar is taken as the total error $(\mathrm{X})$ shown on Figure 4B and tabulated in Table 4. The lines with whole-minute logging of the shot-time calibration points are ovvious because of their large error bars (e.g., lines 1-8).

A quality rating has been assigned to each line based on the size of the total error and is illustrated in Figure 6B. The lines have been arranged in order of increasing error-bar size. Lines with error bars of 6-12 $\mathrm{m}$ are considered to have excellent navigation; those with error bars of 12-24 m (i.e., up to half a shot interval) are considered to have good navigation; and those with error bars in excess of $50 \mathrm{~m}$ (i.e., one shot interval) are considered to have poor navigation.

\section{Line Crossings}

Line crossing information has been compiled from the processed navigation locations for each multichannel line and is given in Appendix 3.

\section{DISCUSSION}

A map of the navigation quality rating of each line (Figure 7) shows that lines on the southern side of the Selenga Delta have the worst ("Poor") navigation whereas those around Academician Ridge have the best ("Excellent") navigation. The reason for this geographic distribution can be explained because the Selenga Delta survey was completed first, when the most communication problems and human error occured in logging.

In as much as the purpose of the cruise was to collect multichannel seismic data, a relevant issue is how the quality of the navigation data affects the multichannel data, specifically the multichannel geometry. The marine multichannel technique is based on repetition of regular geometries that allow sorting and reconstruction of common depth point seismic gathers for velocity analysis and stack (e.g., Yilmaz, 1987). Perturbations of this geometry, such as that which occurs when shots are not at the target 50-m spacing, introduce artifacts and complications in processing the data.

For the Baikal data, the shots were ideally 50-m apart to preserve the optimum geometry for recovering 24-fold data (Agena et al., 1994). The actual mean distance between shots ranged from $45 \mathrm{~m}$ (line 14) to $57 \mathrm{~m}$ (line 30). For all lines except line 30, the uncertainty (i.e., the scatter around the mean shot spacing) includes $50 \mathrm{~m}$, and the assumption of 50-m firing intervals for processing the data is therefore within the estimated navigational uncertainty.

Because of the low frequencies used in the multichannel seismic survey (less than $60 \mathrm{~Hz}$ ), velocity estimations will not be affected by using a geometry of 50-m firing intervals instead of the actual mean intervals of between 45 and $57 \mathrm{~m}$. The region insonified on the lake floor by 


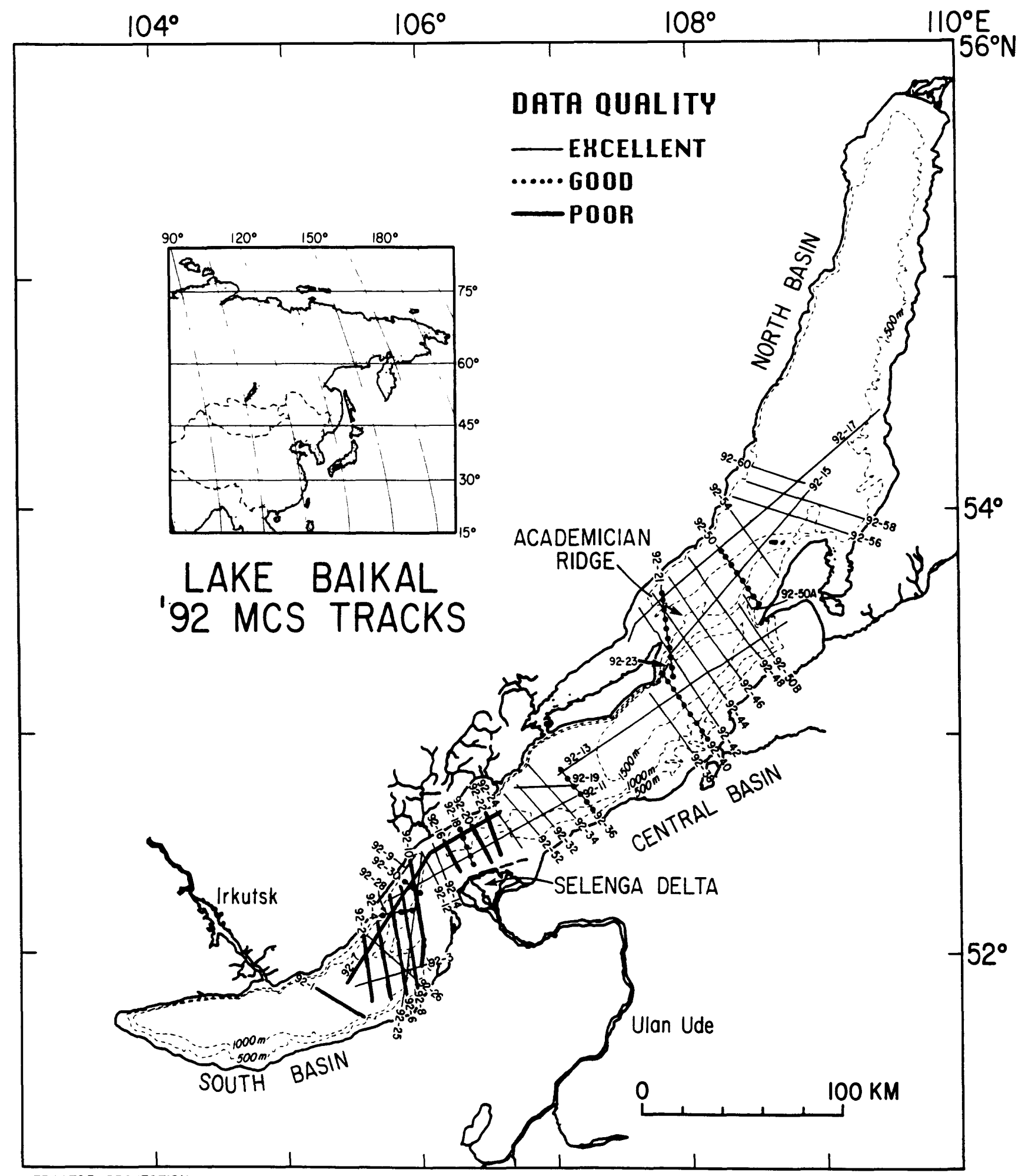

MERCATOR PROJECTION

Figure 7. Map showing the distribution of the navigation quality for the lines in Lake Baikal. The lines with the lowest "Poor" rating are generally in the region of the south Selenga Delta, where shot-time calibration logging was poorest at the beginning of the cruise. 

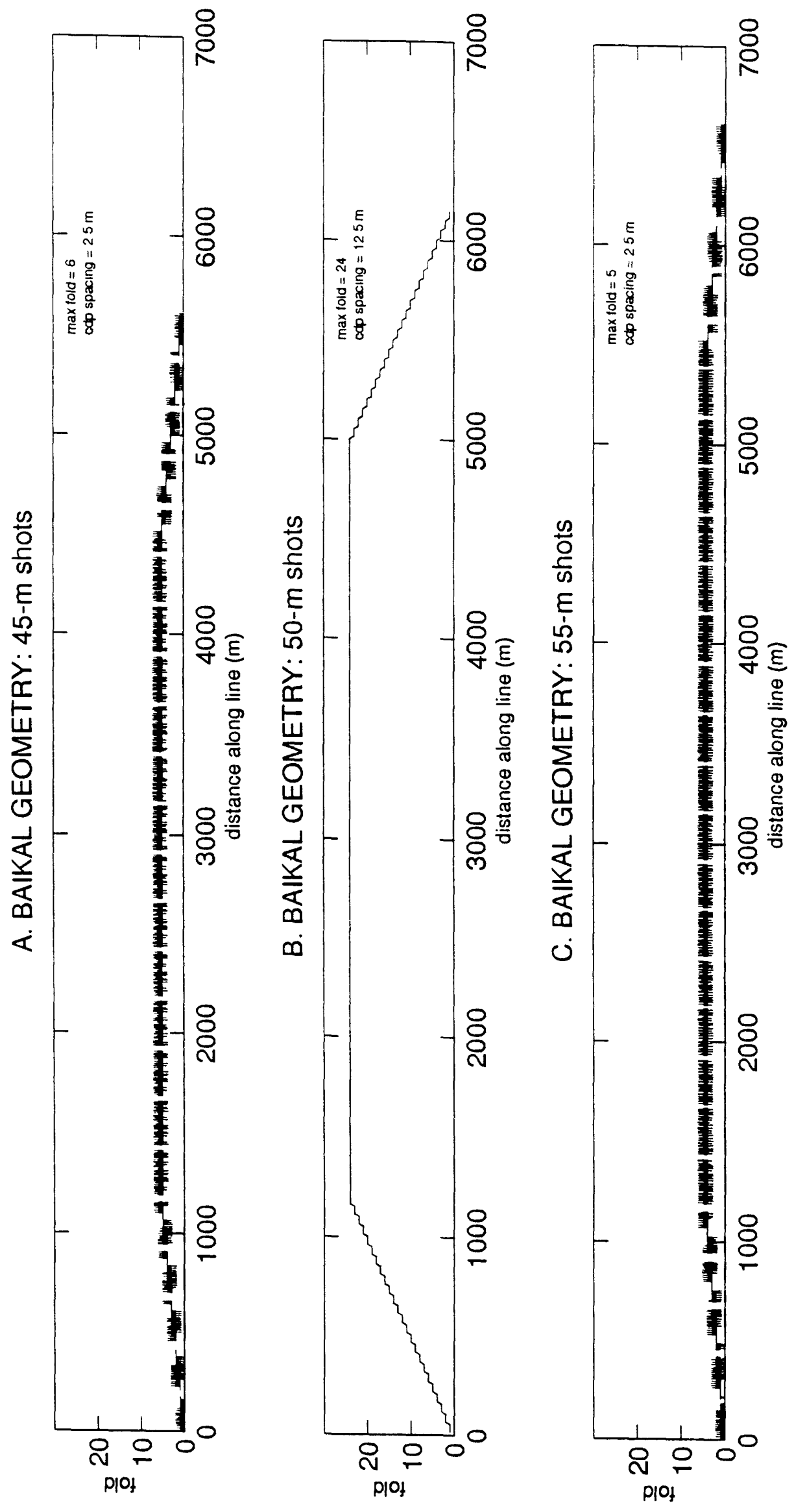

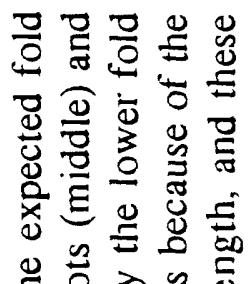

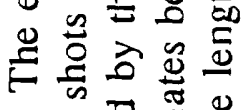

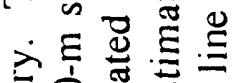

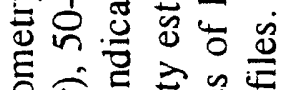

产席.

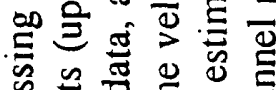

岁 合

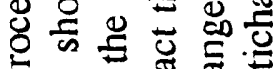

타

宁

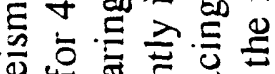

语

व छ

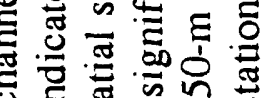

可的

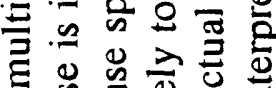

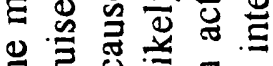

Е 己

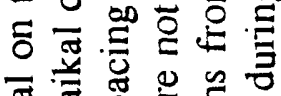

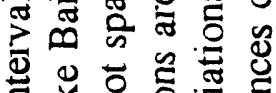

㟧

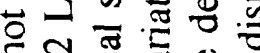

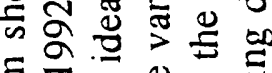

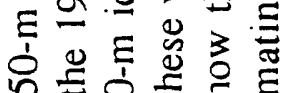

$\subseteq$ 员

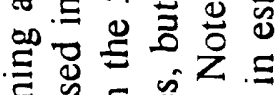

ज绻它

तो

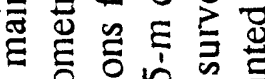

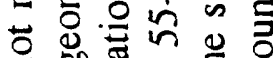

용.

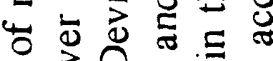

U

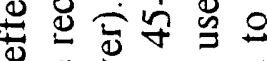

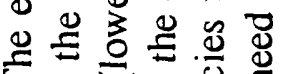

$=2=\frac{9}{0}$

원

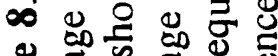

娄的

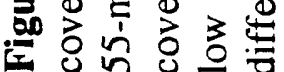


the seismic signal, i.e., the fresnel zone, at these frequencies is on the order of tens of meters. Shot intervals up to $7 \mathrm{~m}$ different than the ideal interval (i.e., $57-\mathrm{m}$ shot intervals) would be inconsequential in velocity calculations.

A consequence of the navigational uncertainties is that some horizontal smearing of the data will occur, particlulary for lines where the mean firing rate differs from $50 \mathrm{~m}$ by more than $5 \mathrm{~m}$. Figure 8 shows the fold coverage calculated using the Baikal multichannel source-receiver configuration (Agena et al., 1994) for 100 shots at 3 firing intervals: $45 \mathrm{~m}$ (top), $50 \mathrm{~m}$ (middle), and $55 \mathrm{~m}$ (bottom). In the ideal case $(50 \mathrm{~m}$ ), the fold coverage increases from 0 to 24 over common depth point (cdp) intervals of $12.5 \mathrm{~m}$. For the $45-\mathrm{m}$ case, the fold increases in a jerky pattern up to 6 , and fluctuates between 4 and 6 for the bulk of the line with a cdp interval of 2.5 $\mathrm{m}$. Similarly, the 55-m case only reaches maximum fold of 5 and fluctuates between 3 and 5 , also with a cdp interval of $2.5 \mathrm{~m}$. Merging 4 adjacent cdps from the $45-\mathrm{m}$ and $55-\mathrm{m}$ cases would produce $12.5-\mathrm{m}$ binned cdps containing 24 traces, similar to the ideal $50-\mathrm{m}$ case. The result is spatial smearing of the data horizontally. The low frequencies and size of the fresnel zone render most of this smearing inconsequential. However, the effect will be most pronounced in regions of steep relief, which are common in Lake Baikal along Academician Ridge and at the sides of the lake.

Another consequence of the navigational uncertainty is miscalculation of total line length and distances between cdps on the multichannel profiles. Figure 5 shows that the total line length for the line shot with $45-\mathrm{m}$ intervals is $5645 \mathrm{~m}$, whereas it is $6150 \mathrm{~m}$ and $6635 \mathrm{~m}$ for the $50-\mathrm{m}$ and $55-\mathrm{m}$ cases respectively. Distances measured off the seismic profiles should be corrected for the mean shot interval. Table 1 gives the best estimates of mean shot interval and line lengths for each line in Baikal.

The two missed shots associated with each tape change during the second half of the cruise require a small geometry correction in sorting and stacking the multichannel data. A comparison between a part of line 13 processed with and without the missed shots (Figure 9) shows that the character of the seismic data is essentially unchanged, but that certain details of the profile, for example, offset and overlap along a fault surface (arrow), differ. The fault trace that lines up with the arrow is sharper and more distinct on the profile with the corrections (Figure 9B). This emphasizes the importance of accurate navigation for processing of the multichannel data, particularly in structurally complex regions. 


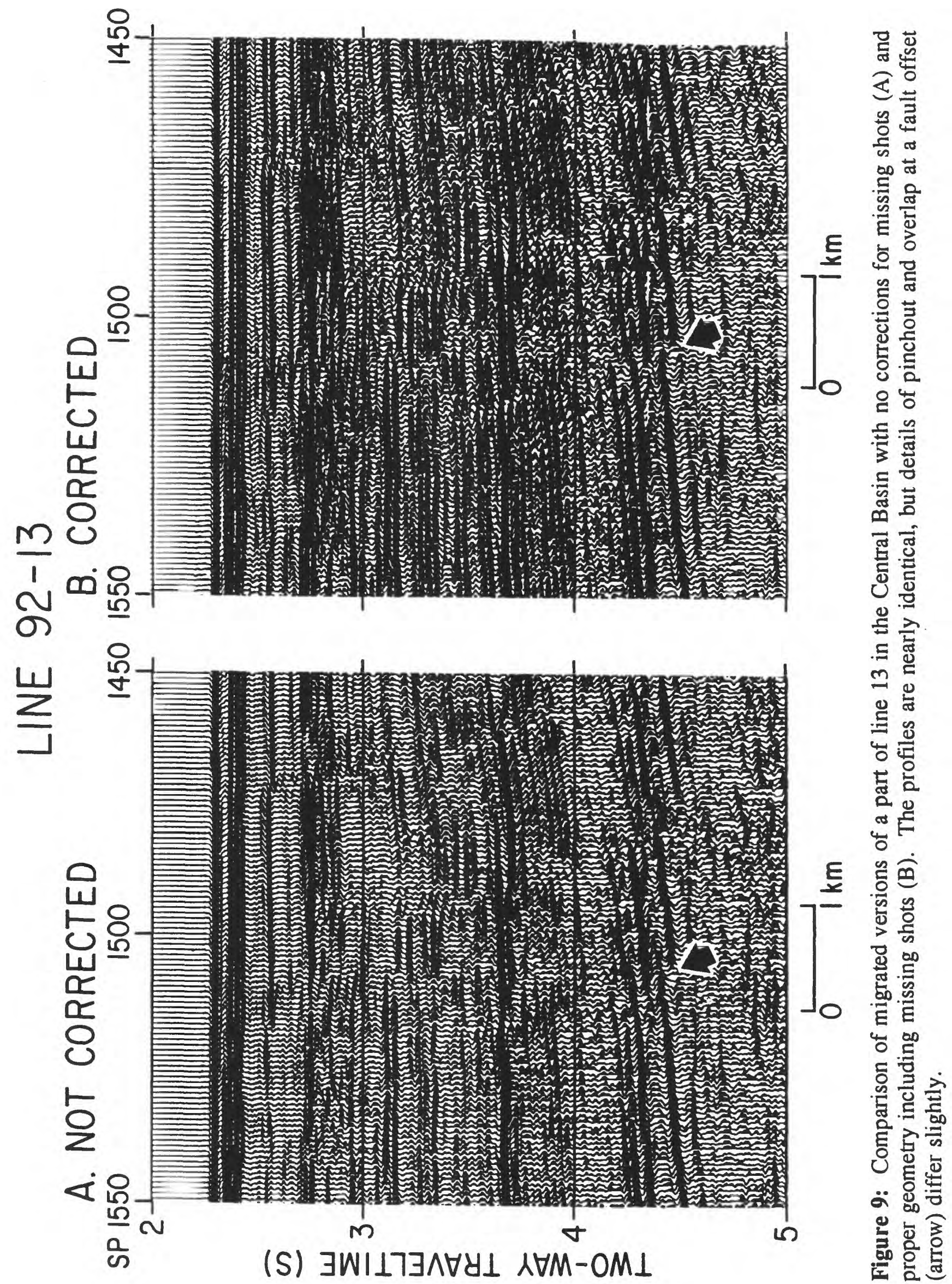




\section{CONCLUSIONS}

The navigation data collected on the 1992 Lake Baikal multichannel seismic reflection cruise posed a challenge to processing because of difficulties in assigning times to shot points. The salient conclusions that have resulted from this study are:

(1) Three sources contributed to uncertainty in navigation: inherent measurement limitations, poor logging of the shot-time calibration points, and missing shots. The measurement error varies from $6-22 \mathrm{~m}$. The error associated with shot-time logging ranges from about $2 \mathrm{~m}$ (whole second logging) to $70 \mathrm{~m}$ (whole minute logging). Error associated with the missing shots is greatest at the position of the missed shots and decreases toward the shottime calibration points.

(2) The scatter associated with the raw GPS data varies between lines and can be very noisy ( $>10 \mathrm{~m}$ per each $10-\mathrm{s}$ interval) or very quiet ( $<2 \mathrm{~m}$ per each $10-\mathrm{s}$ interval). Most of the scatter in the raw navigation is a function of variations in HDOP value and reflects uncertainty introduced by changing satellite geometries used to compute successive locations.

(3) After careful editing and processing of the raw navigation and calibration files, the quality of each line was assigned a rating of:

$\begin{array}{lcl}\text { Excellent: } & 28 \text { lines } & 6-12 \mathrm{~m} \text { uncertainty } \\ \text { Good: } & 7 \text { lines } & 12-24 \mathrm{~m} \text { uncertainty } \\ \text { Poor: } & 13 \text { lines } & >50 \mathrm{~m} \text { uncertainty }\end{array}$

(4) The lines rated "Poor" generally occur in the southern Selenga Delta area. This is because the southern Selenga Delta was the first region profiled and includes the largest shot-time calibration errors.

(5) The assumption of a 50-m firing interval used to process the multichannel seismic data is valid and within the navigational uncertainty associated with shot locations. Actual deviations from $50 \mathrm{~m}$ are not likely to affect velocity estimates, but will cause some spatial smearing of the data, particularly in regions of steep relief. Distances measured off the seismic profiles should be corrected for actual shot distances of each line, rather than the assumed distance of $50 \mathrm{~m}$. 


\section{REFERENCES CITED}

Agena, W.F., Lee, M.W., Miller, J.J., and Hutchinson, D.R., 1994, Lake Baikal - 1992 Processing of Multichannel Seismic Reflection Data: U.S. Geological Survey Open-File Report OF-94-263, 44 pp.

Hurn, J., 1989, GPS - A guide to the next utility: Trimble Navigation, Sunnyvale, California, p. $1-47$.

Klitgord, K.D., Golmshtok, A. Ja, Scholz, C.A., Akentiev, L., Nichols, D., Schneider, C., McGill, J., Foster, D., and Unger, D., 1993, Seismic Survey of Lake Baikal, Siberia - Cruise Report: RV Balkhash 25 August to 25 September 1992: U.S. Geological Survey OpenFile Report OF-93-201, 25 pp.

Milliken, R.J., and Zoller, C.J., 1980, Principle of operation of NAVSTAR (GPS) and system characteristics, in Janiczek, P.M., ed., Global Positioning System: The Institute of Navigation, Washington, D.C., p. 3-14.

Nichols, D.R., Miller, G., and Akentiev, L., 1992, Seismic survey of Lake Baikal, Siberia: Operational technical summary for the RV Balkhash and RV Titov 15 August to 30 September 1992: U.S. Geological Survey Open-File Report OF-92-693, 21 pp.

ten Brink, U.S., Badardinov, A., Miller, G.K., and Coleman, D.F., 1993, Ocean Bottom Seismometers operation during the seismic survey of Lake Baikal, Siberia, Autumn, 1992: U.S. Geological Survey Open-File Report OF-93-7, 24 pp.

Yilmaz, O., 1987, Seismic data processing: Society of Exploration Geophysicists, Investigations in Geophysics No. 2, 526 pp. 


\section{APPENDIX 1:}

\section{PLOTS OF RAW GPS NAVIGATION}




\section{DESCRIPTION OF PLOTS OF RAW NAVIGATION DATA}

This appendix contains plots of raw navigation from all of the Lake Baikal 1992 multichannel lines, organized by increasing line number. All of the data are plotted against number of hours from the start of the line; because all lines are plotted at the same horizontal scale ( 7 hours), some lines are plotted on more than one page. The GMT start of each line is labelled for each line.

\section{GPS - Distance Between Fixes}

The top plot shows the estimated distance between adjacent (10-second) navigation locations calculated using geodetic inverse formula based on geodesy by A.R. Clarke. Ideally, these plots of distance between fixes should be a flat line (assuming a constant or near constant speed of the ship). Examples of nearly flat plots are line 1, hours 2-3, line 13, hours 9-11). Several reasons can explain spikes on these plots:

(1) data gaps in the 10-second positions will cause abnormally large estimates of distance between fixes; these can be identified by coincident spikes on the plots of time between fixes plotted beneath the distance plots (e.g., line 7, 3.75 hours after the start of the line).

(2) variations in the speed of the ship will cause distance estimates to vary. These kinds of variations should be relatively smooth and of fairly long wavelength (i.e., changes occur over periods of a few minutes and are constant until the next speed adjustment). Changes in the speed of the ship were very small and are not likely to be resolvable on lines where large spikes contaminate the data.

(3) uncertainty in the absolute GPS locations will cause short-wavelength spikes. Most of the spikes seen on the data are due to this uncertainty (e.g., line 1, 0-2 hours, 3-4 hours) and are directly related to the Horizontal Dilution Of Precision (HDOP) value shown in the third plot.

\section{GPS - Time Between Fixes}

The second plot shows the actual time between 10-second positions; spikes on this plot indicate data gaps. Generally, data gaps less than 1 minute (6 fixes) are considered negligible. The positions of data gaps of one minute or more are given in Table 3 of the main paper.

\section{GPS - DOPs}

The third plot show the Horizontal Dilution of Precision (HDOP) value, which refers to the horizontal error associated with the geometry of the satellites used to compute a fix. Each change in HDOP value indicates a different satellite geometry or different set of satellites used to compute a location. These changes in HDOP generally coincide with large scatter in the estimates of "Distance between fixes" (top plot for each line).

\section{GPS - Altitude}

The bottom plot for each line shows ellipsoidal altitude associated with each position. For some lines, altitude was calculated automatically as part of the GPS solution; these lines, at the beginning of the cruise, show large altitude fluctuations (e.g., line 6). For most of the cruise, ellipsoidal altitude was set to $455 \mathrm{~m}$, so that 3-satellite solutions could be used when 4-satellite 
solutions were not available. (Full GPS capability was not implemented in 1992, and therefore 3-satellite solutions were usually required for one or more time intervals each day). The ellipsoidal altitude is a mathematical surface defined as the sum of the geoid (in $\mathrm{m}$ ) and the height above sea level (also in $\mathrm{m}$ ):

$$
\text { Ellipsoidal altitude }=\text { Geoid }+ \text { Height above Sea Level }
$$

Because the lake surface is essentially a constant height $(455 \mathrm{~m})$, and the geoid for the lake is also approximately constant ( $-24 \mathrm{~m}, \mathrm{C}$. Bowin, personal communication), the ellipsoidal altitude should have been specified as $-24+455=431 \mathrm{~m}$. This difference $(24 \mathrm{~m})$ should not have affected the accuracy or quality of the resultant GPS location (B. Irwin, personal communication).

Statistics about time and distance between fixes, HDOP value, and ellipsoidal altitude are plotted at the end of each plot (mean, standard deviation, maximum and minimum values). Note that the standard deviations include the data gaps and may not be representative of the overall smoothness of the line (e.g., line 13). Line statistics are shown at the end of each line (i.e., on the final page for multiple page plots). 

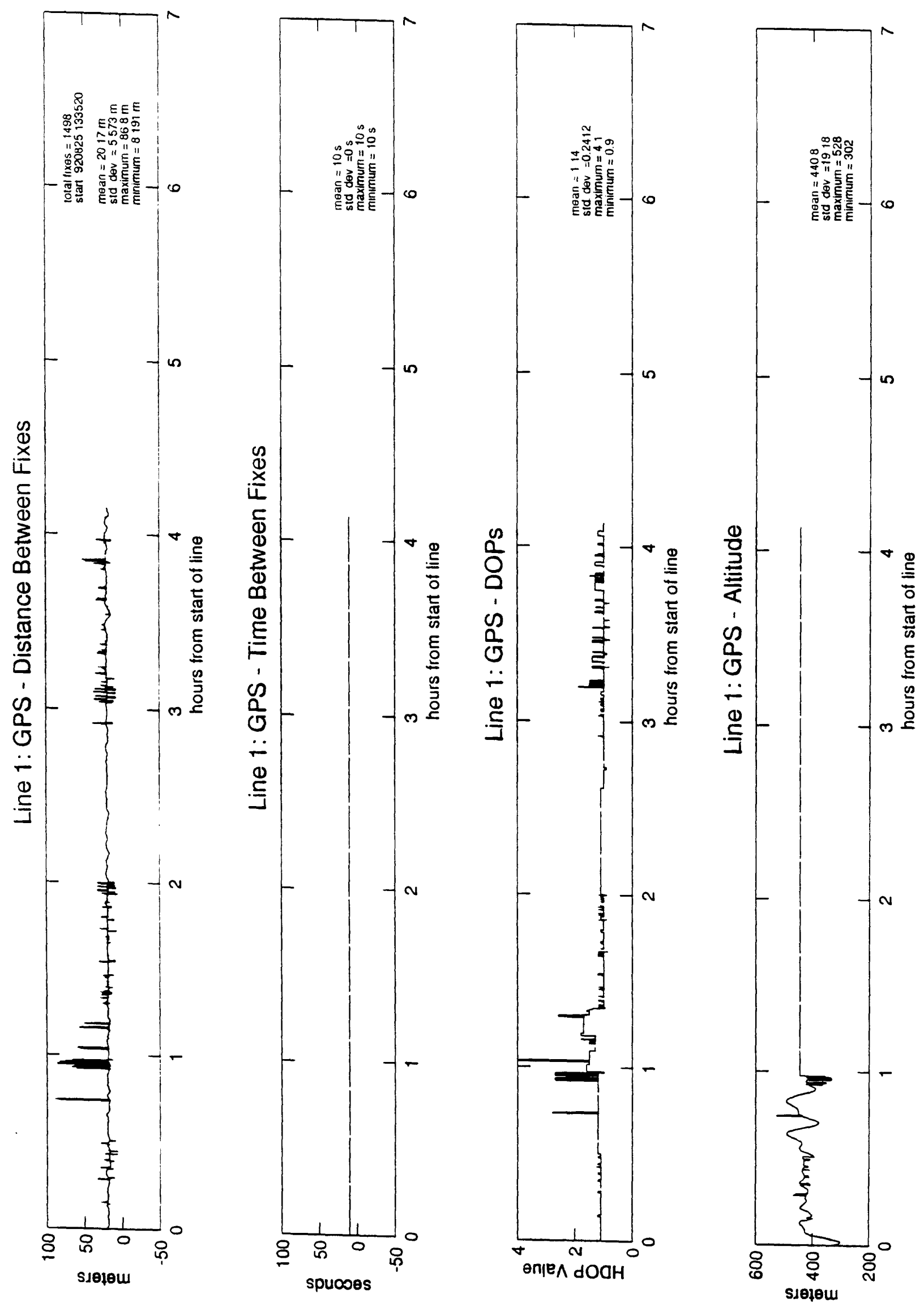

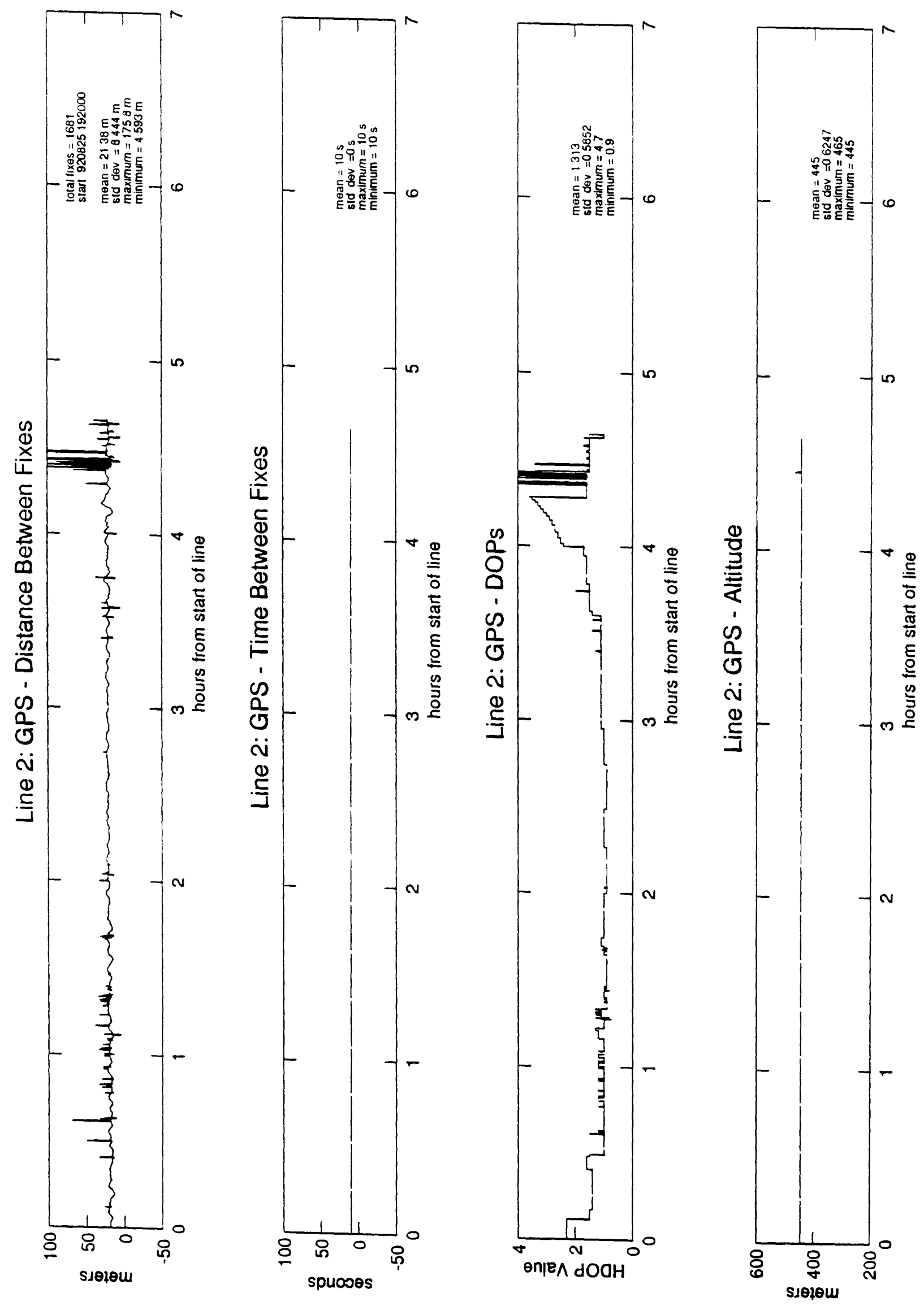


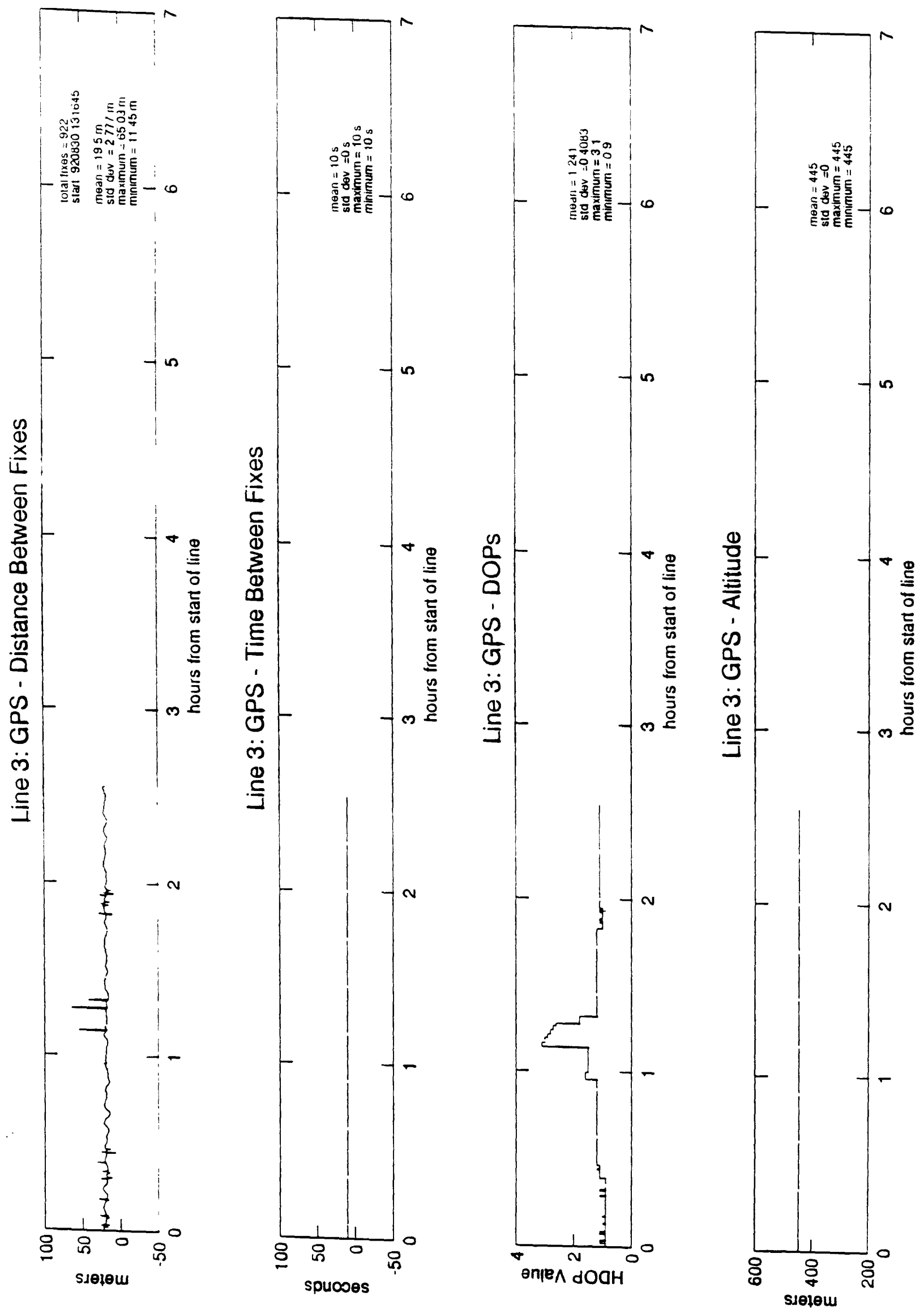



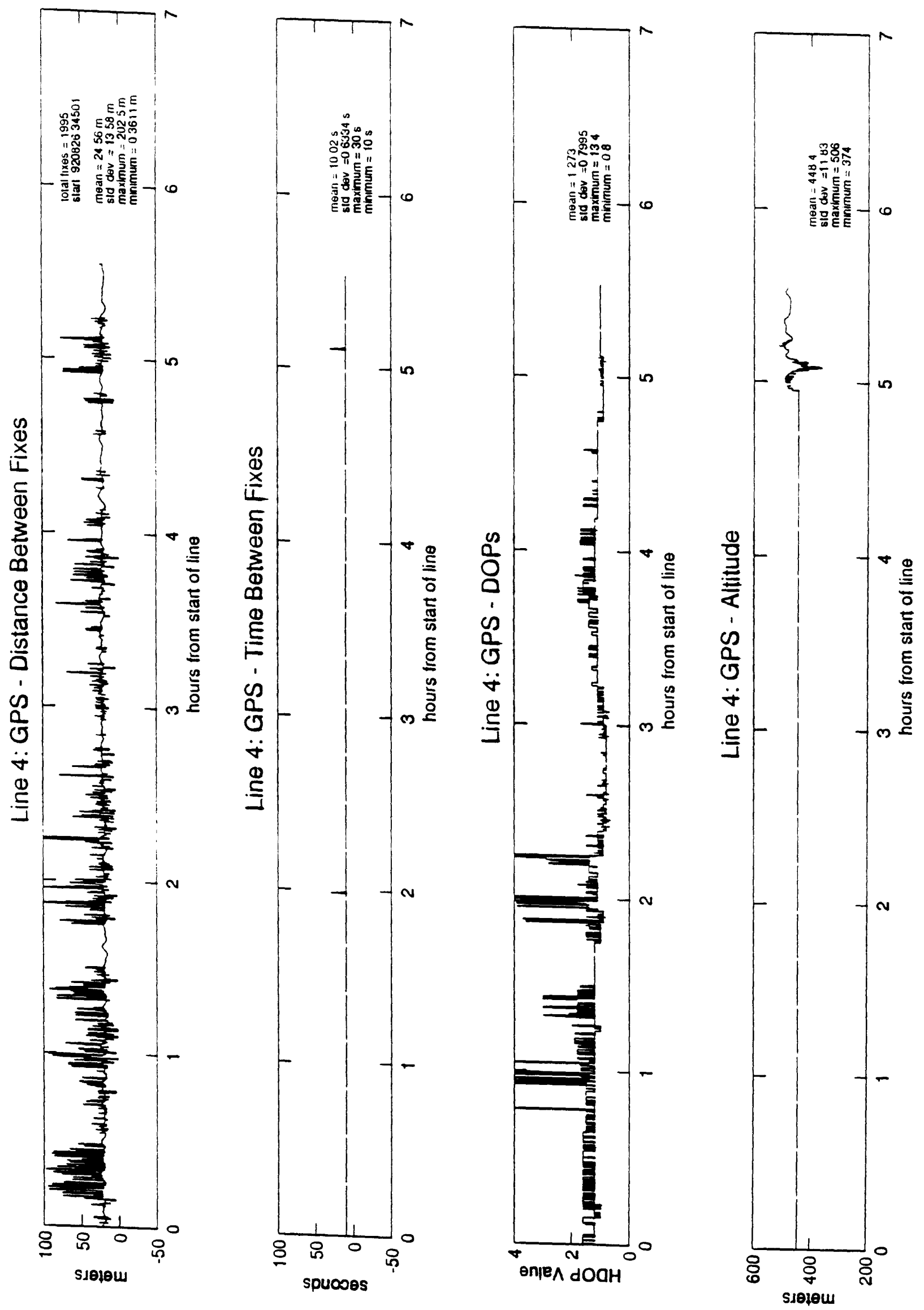


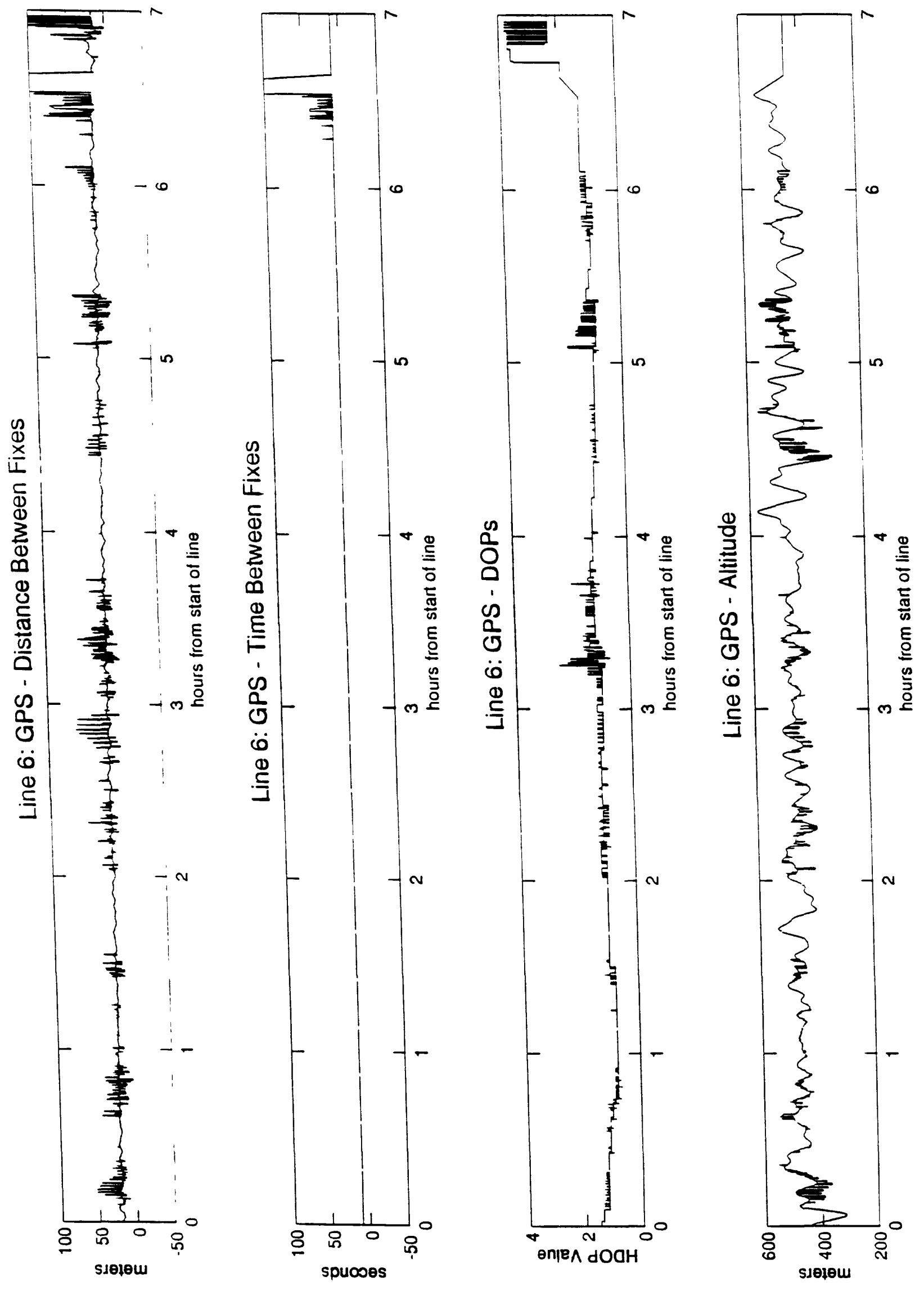




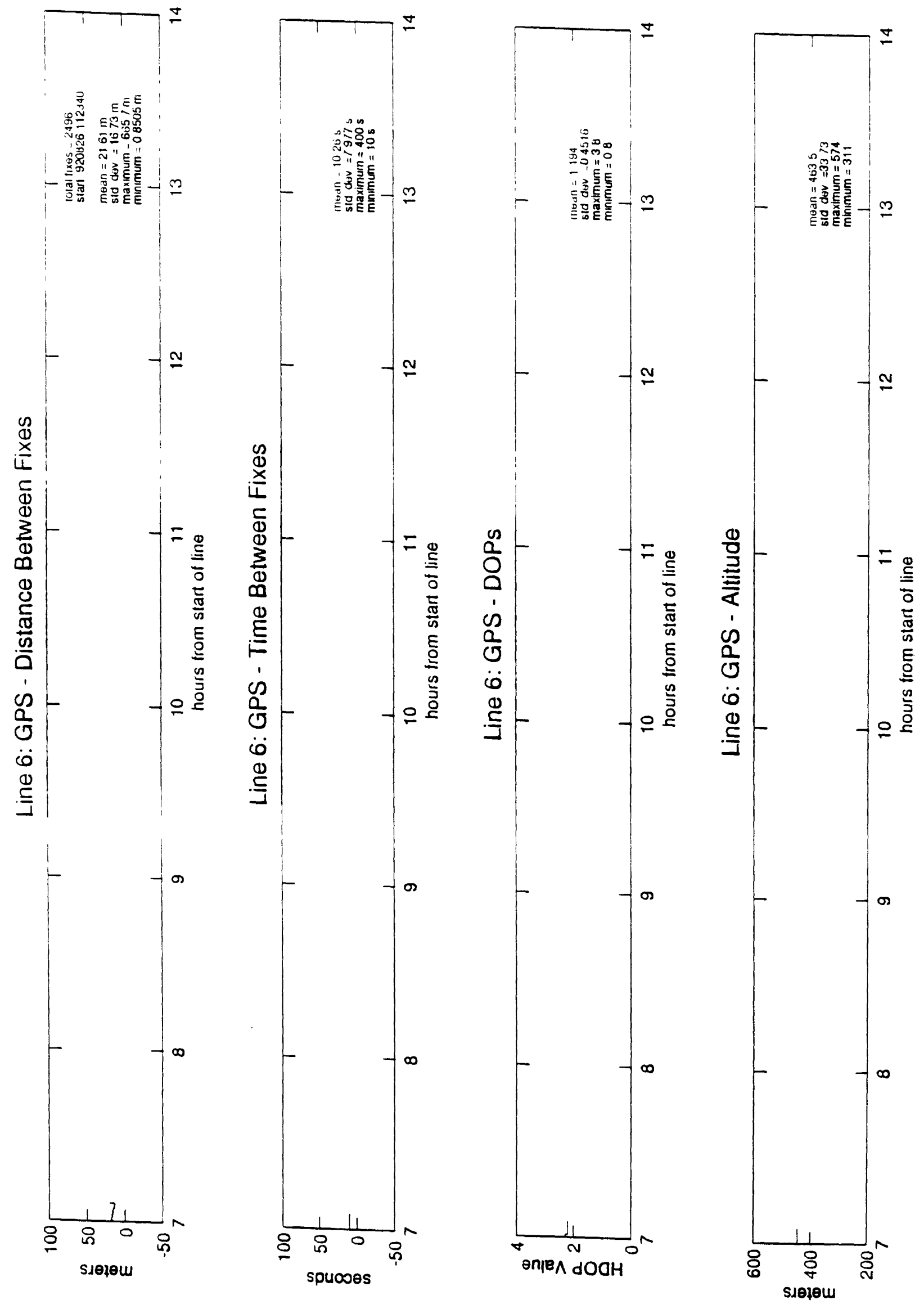




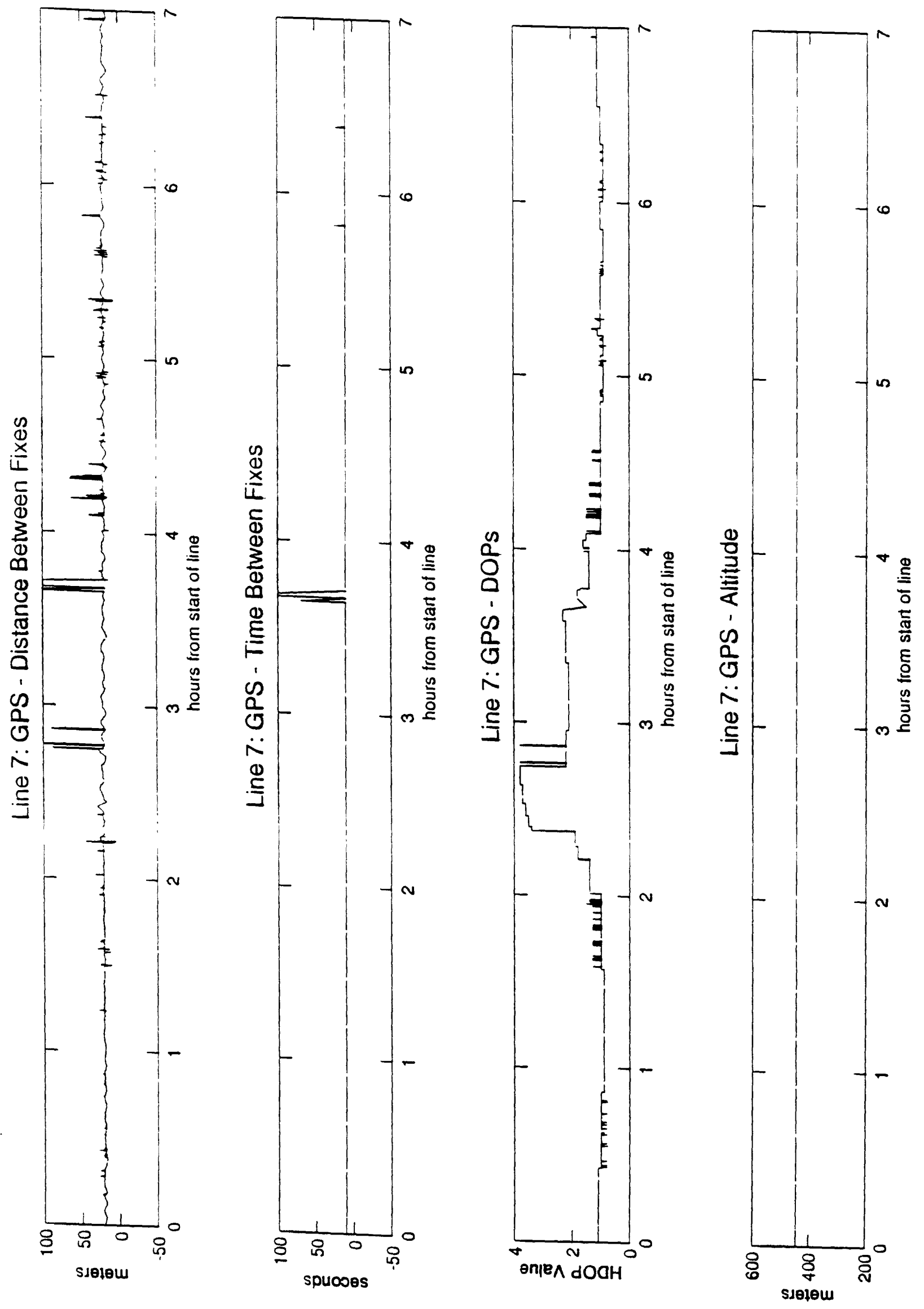



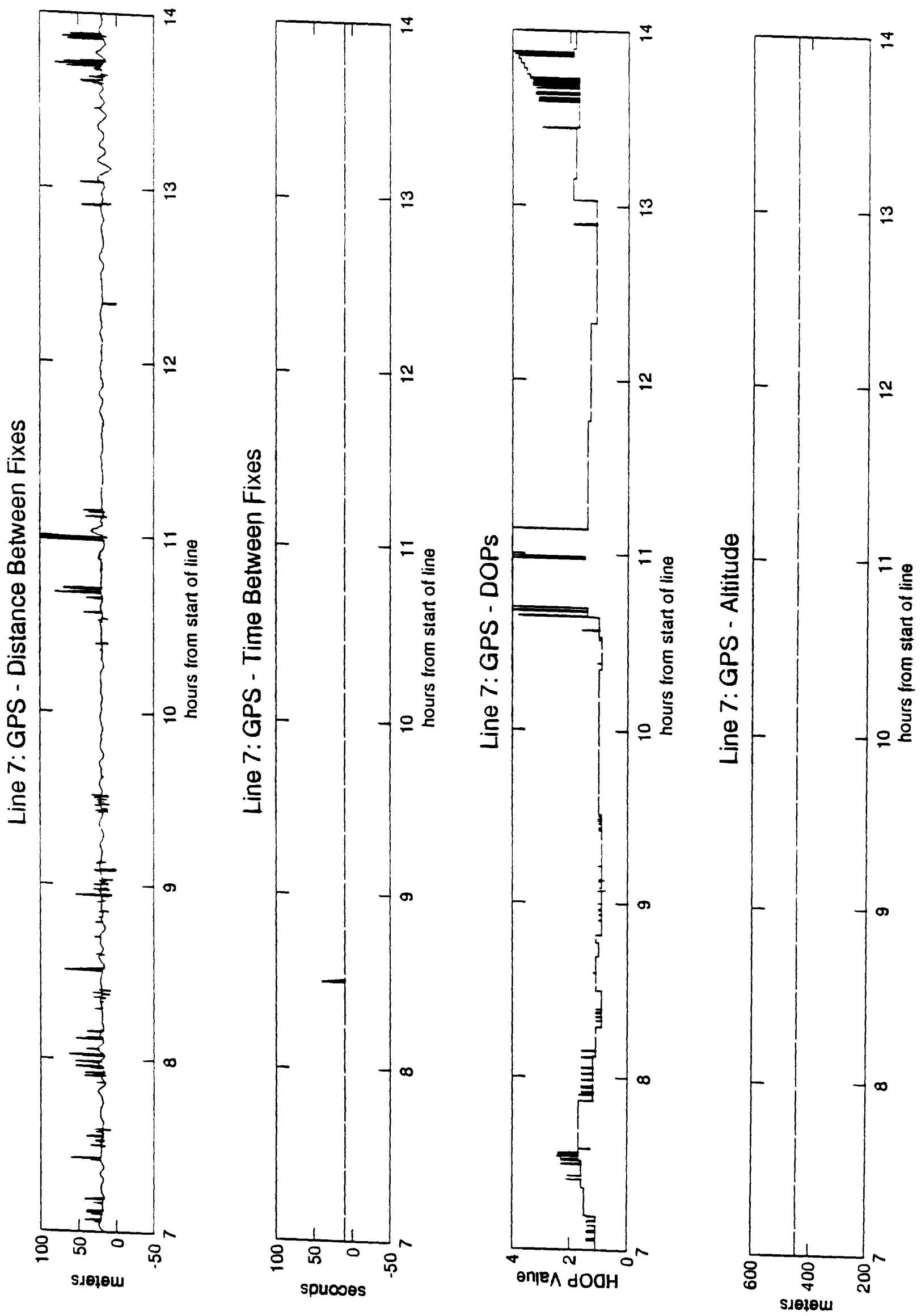

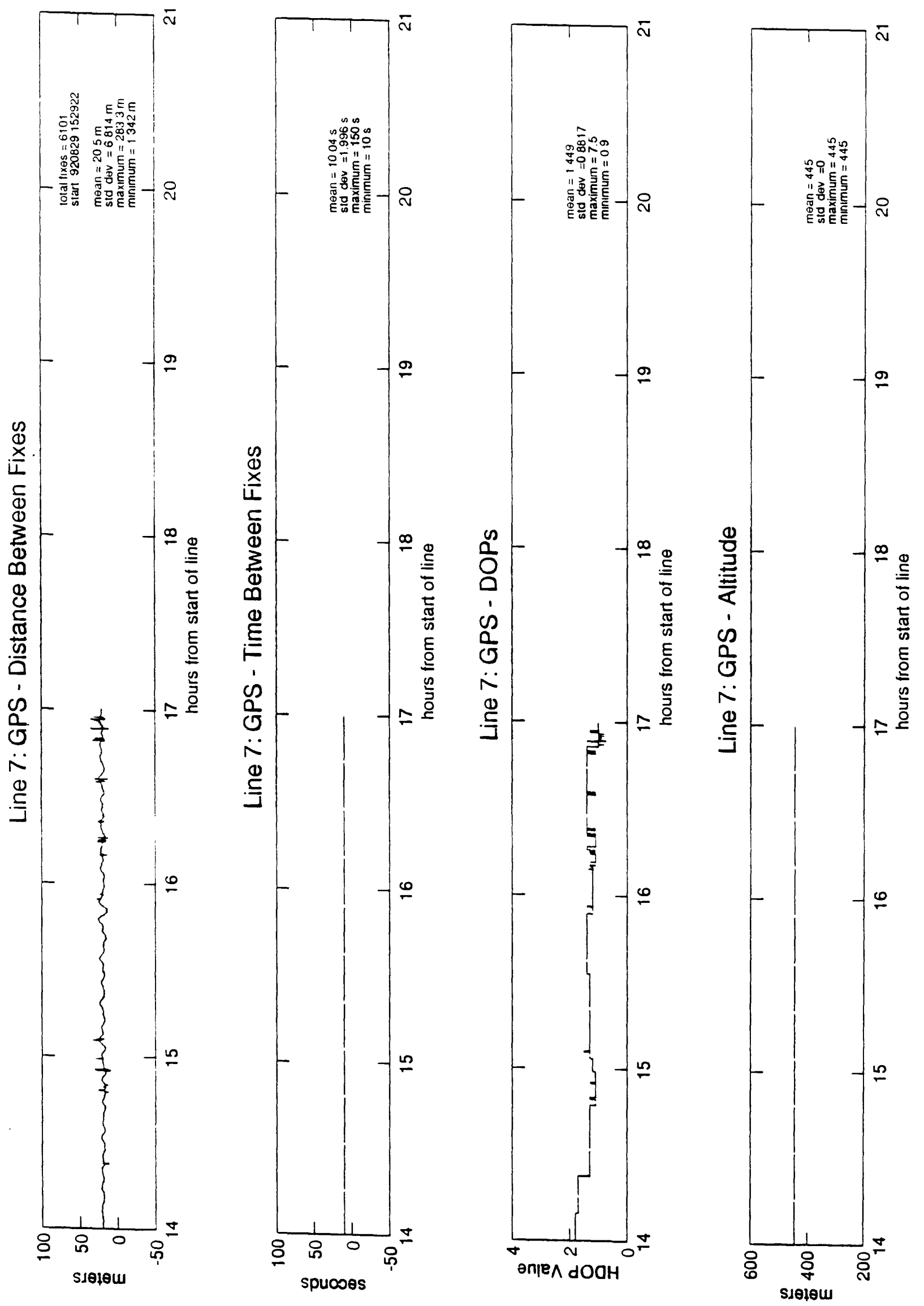

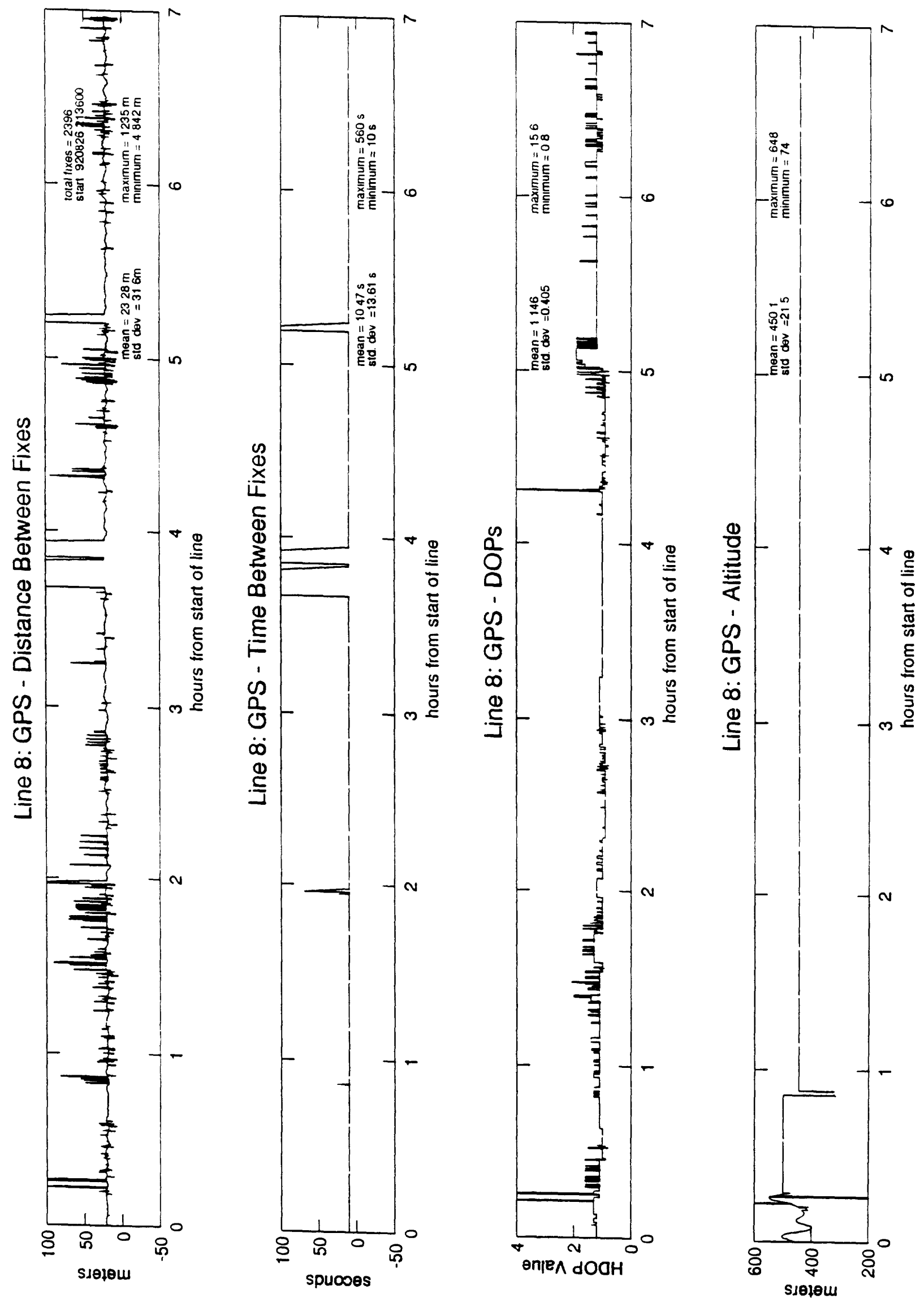

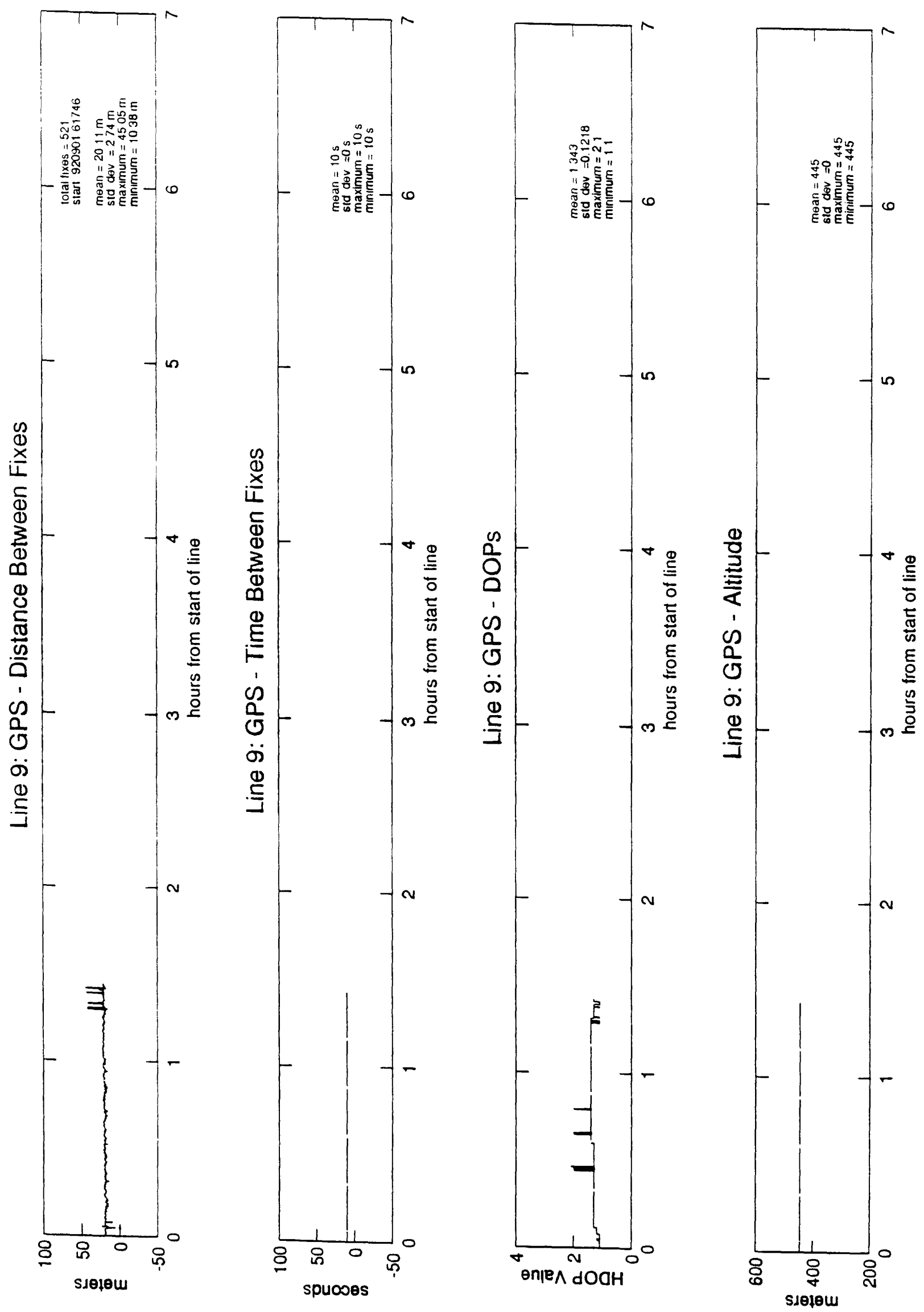

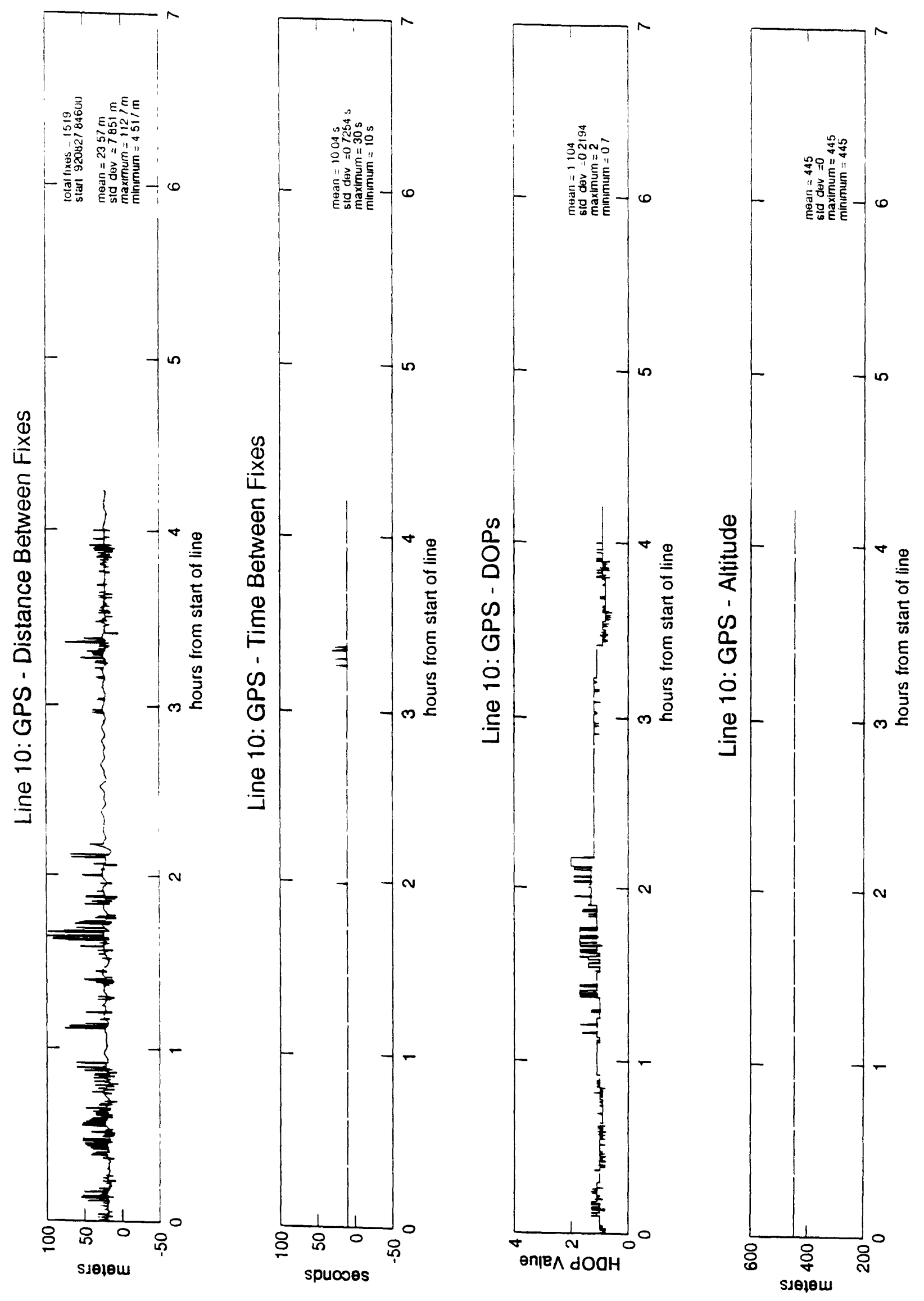

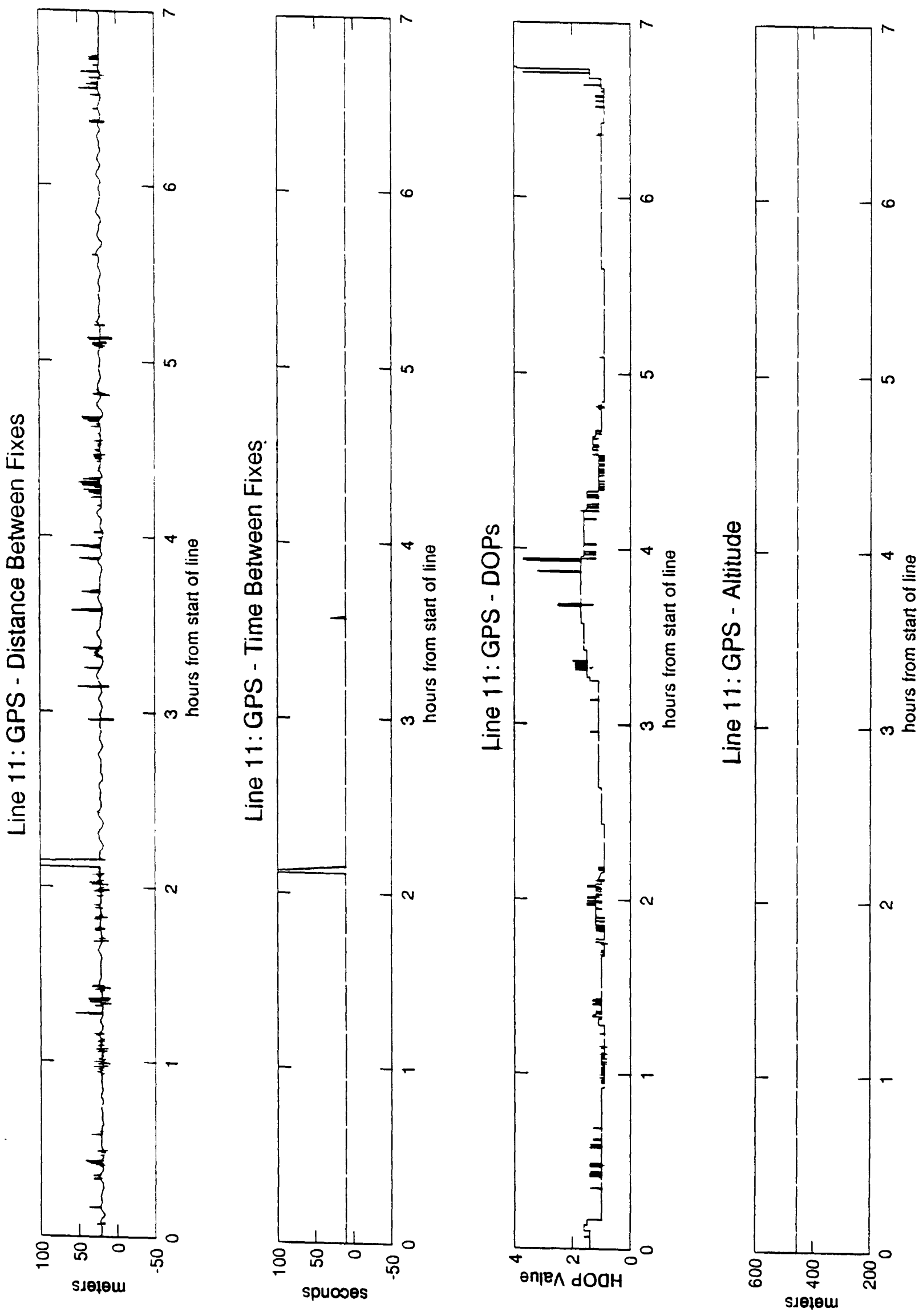

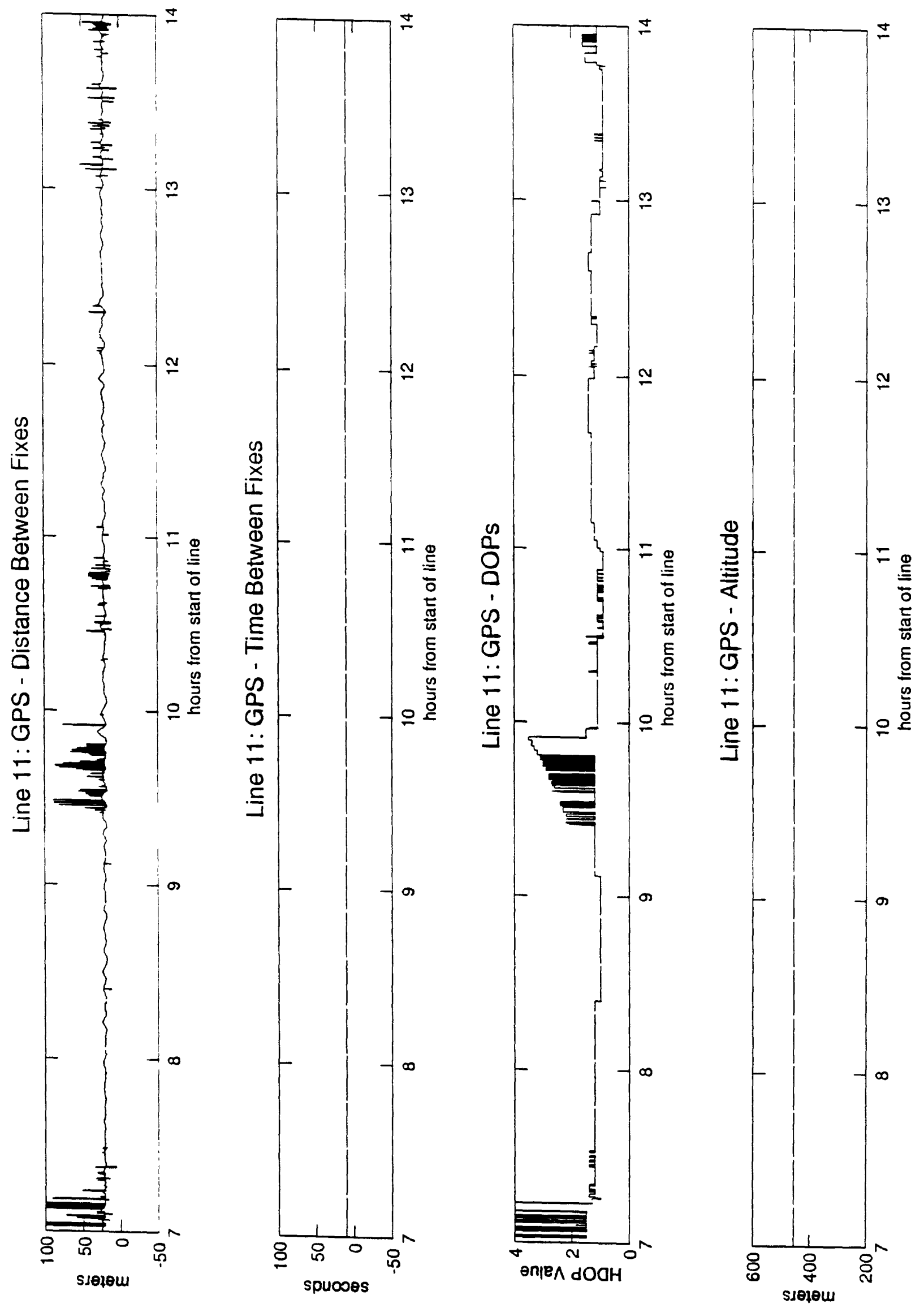

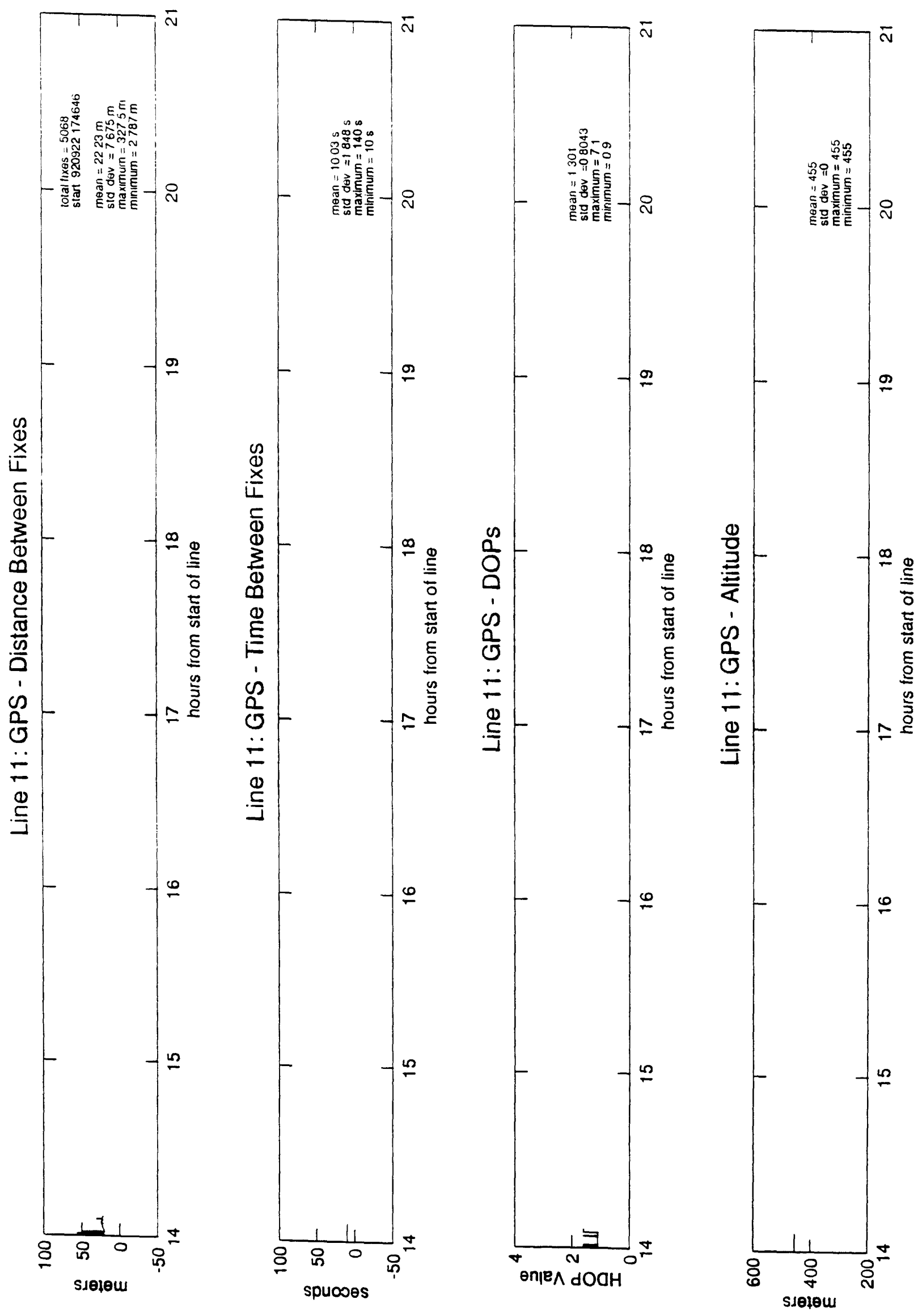

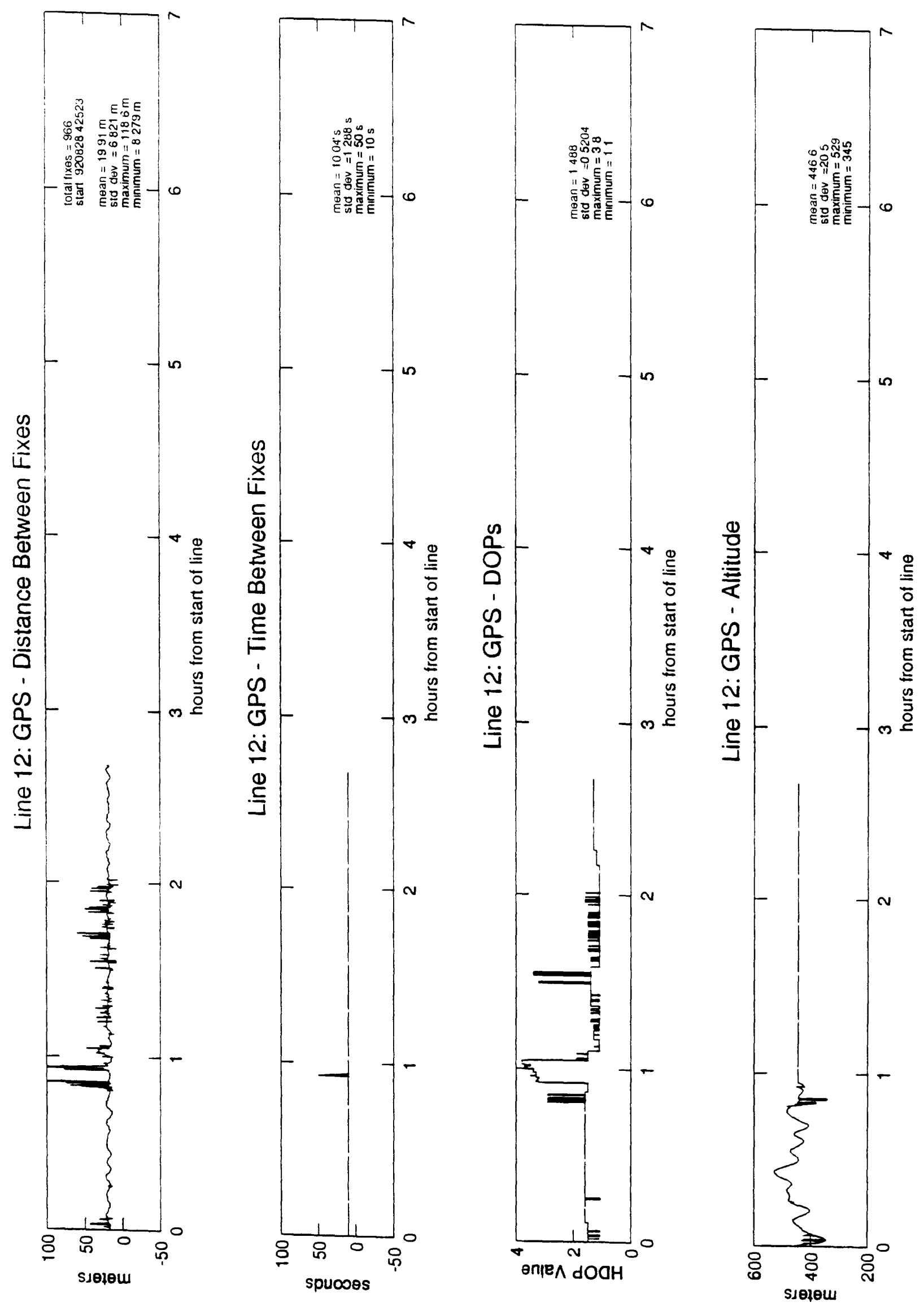

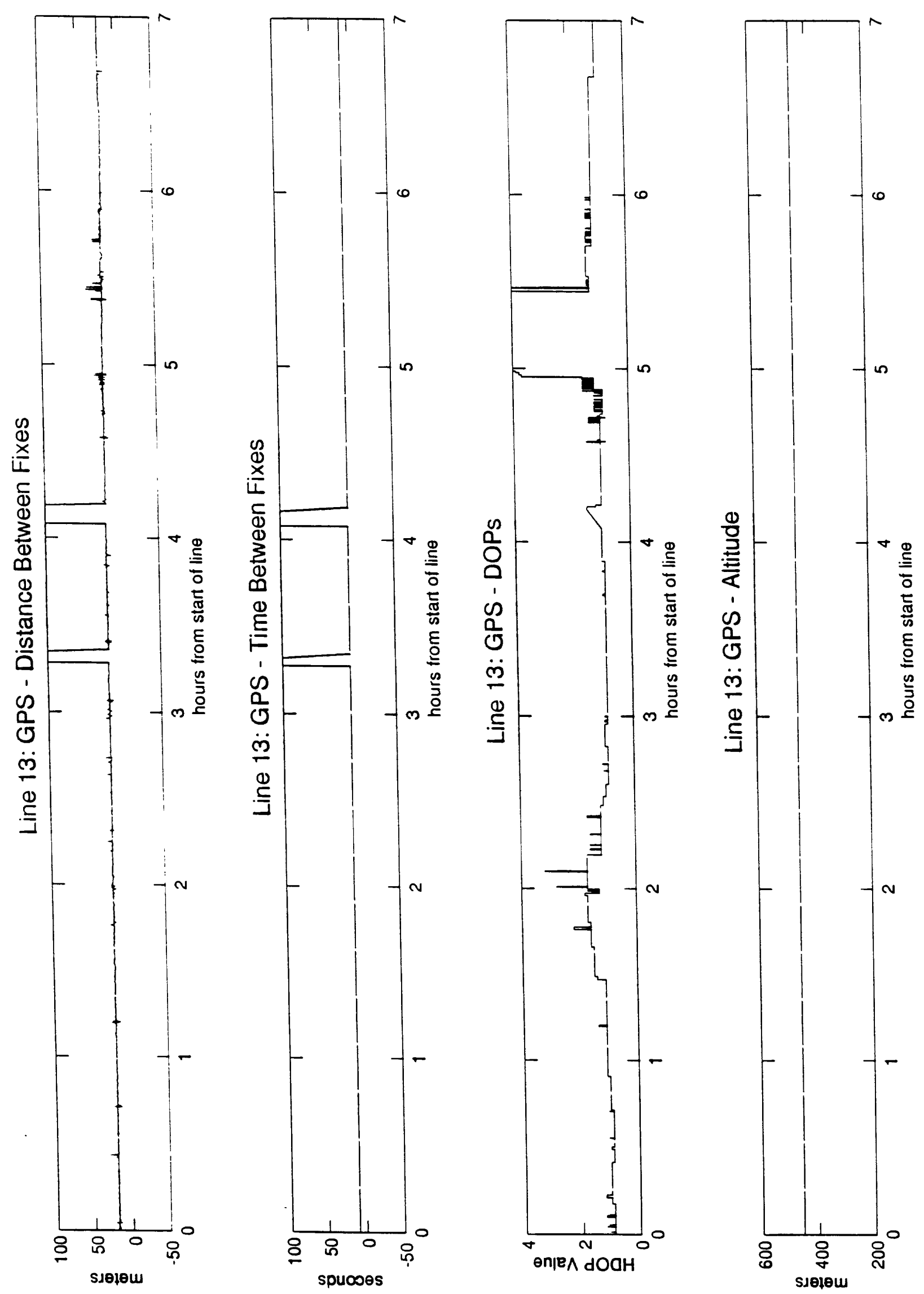

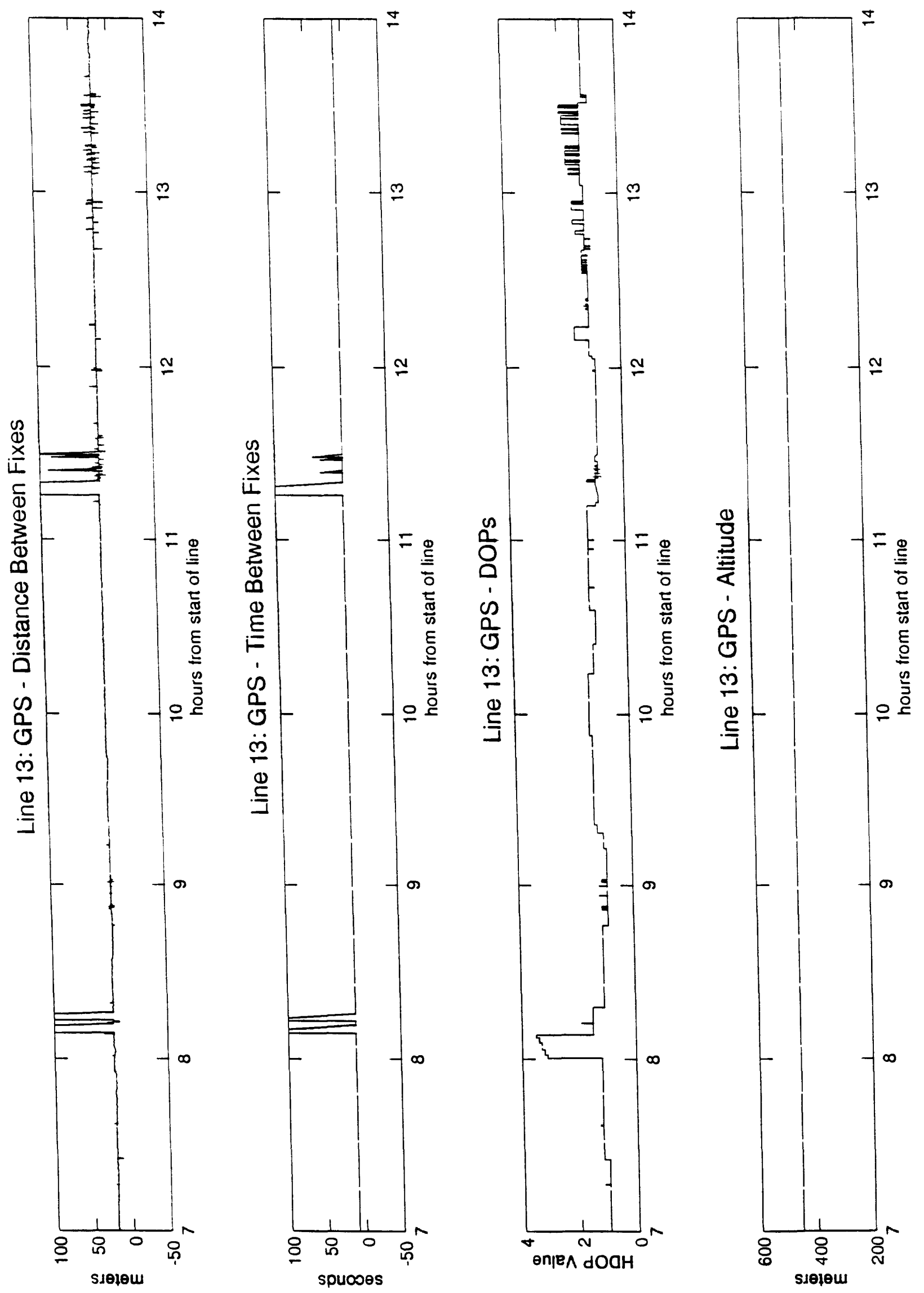

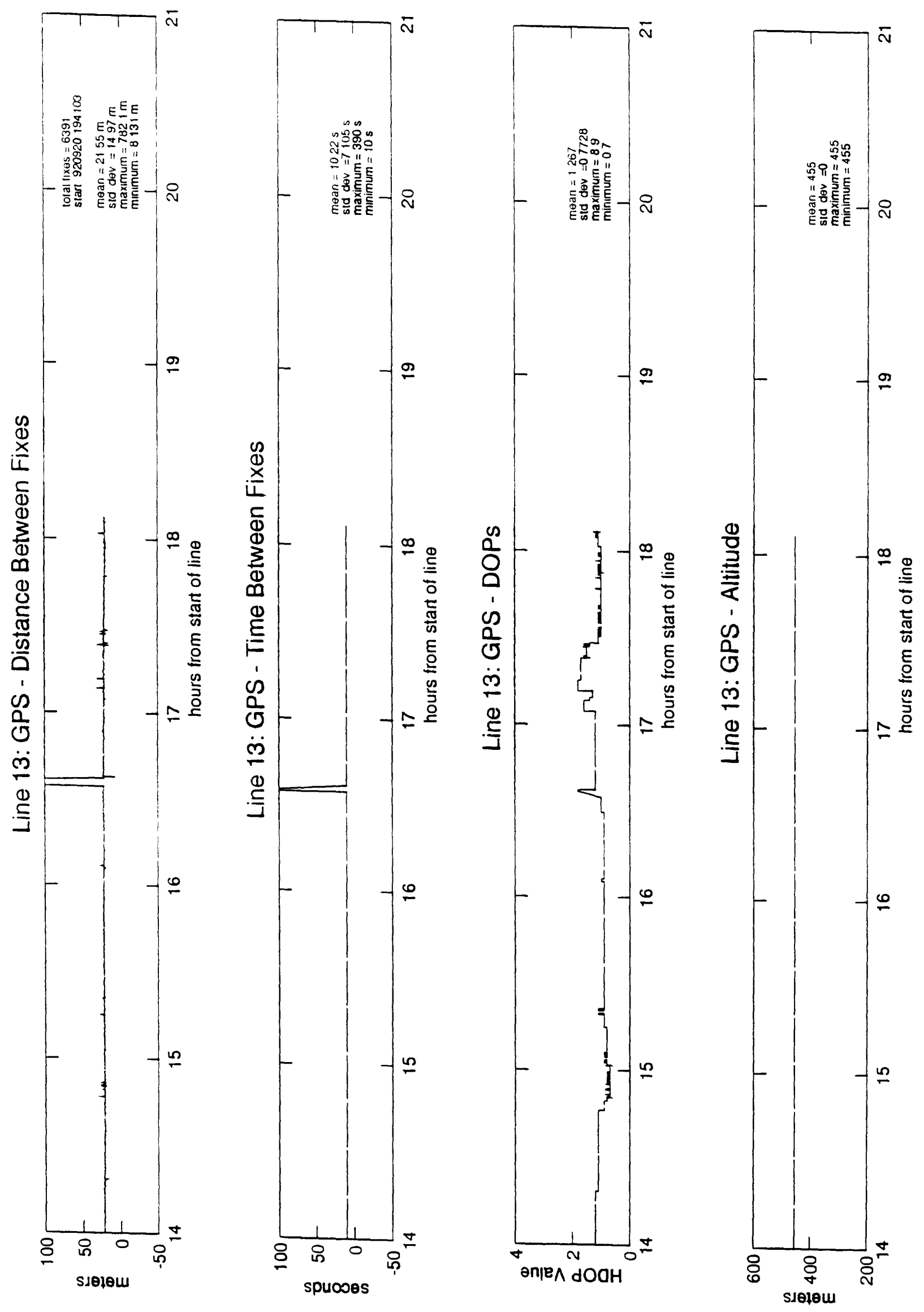

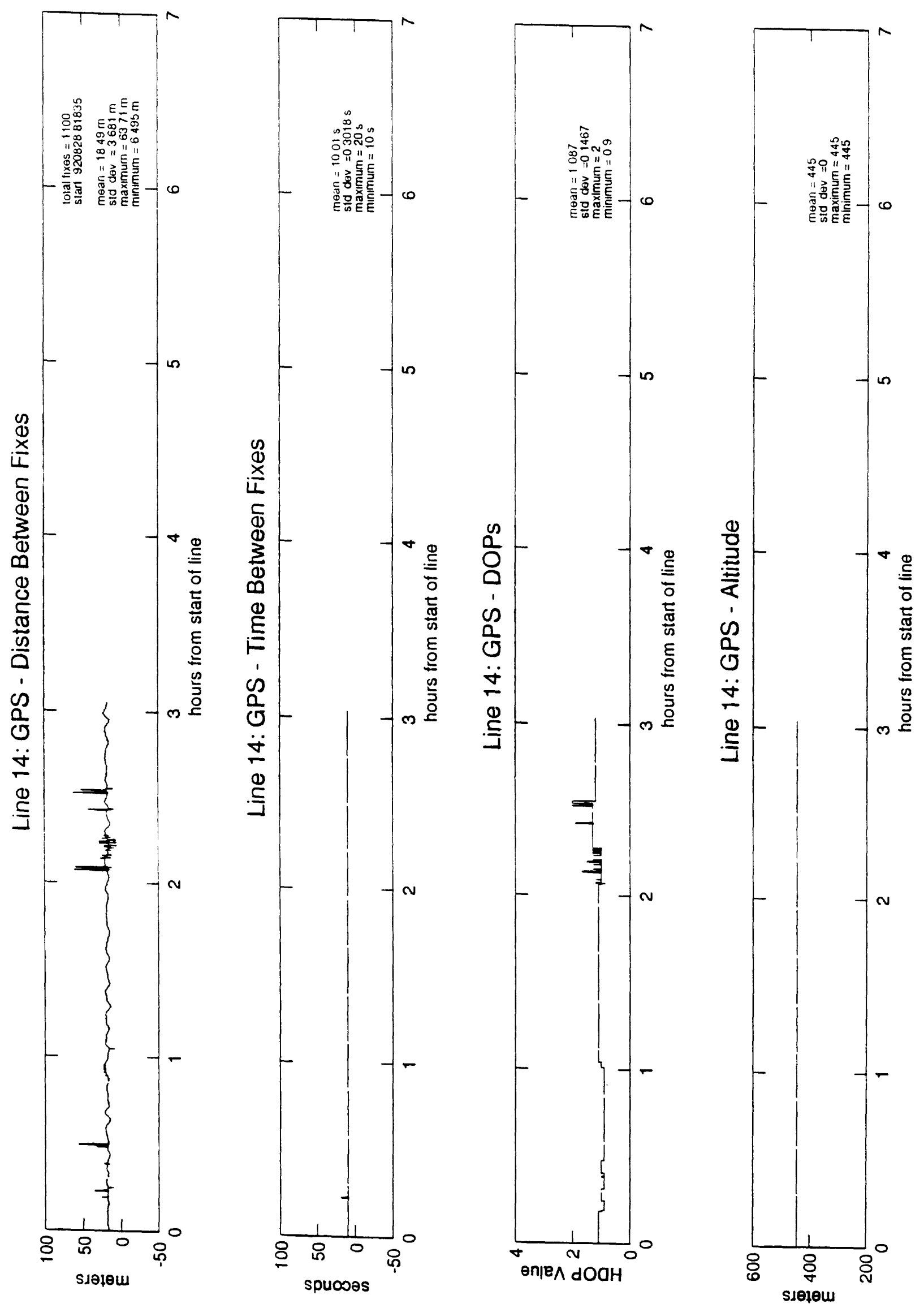


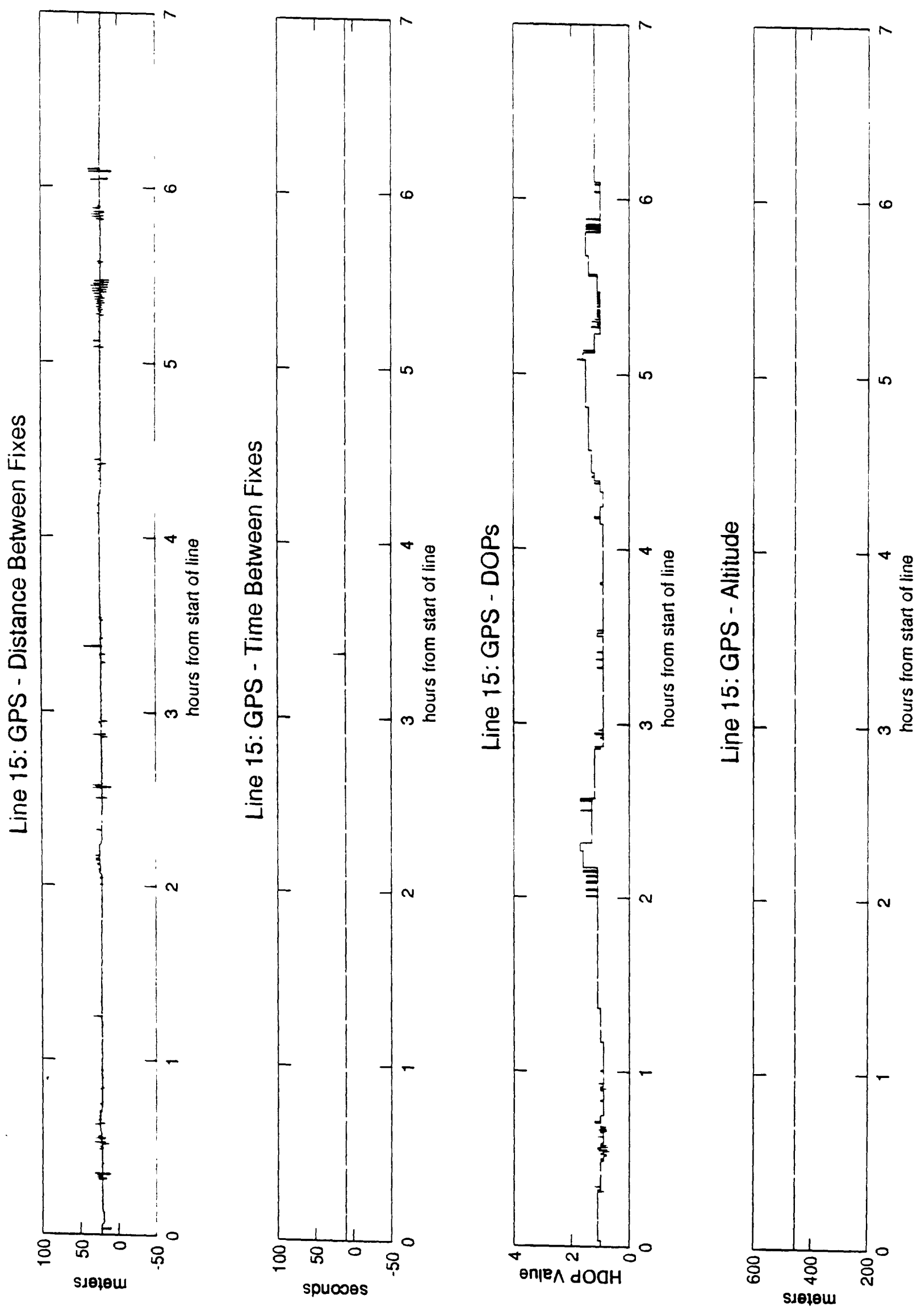




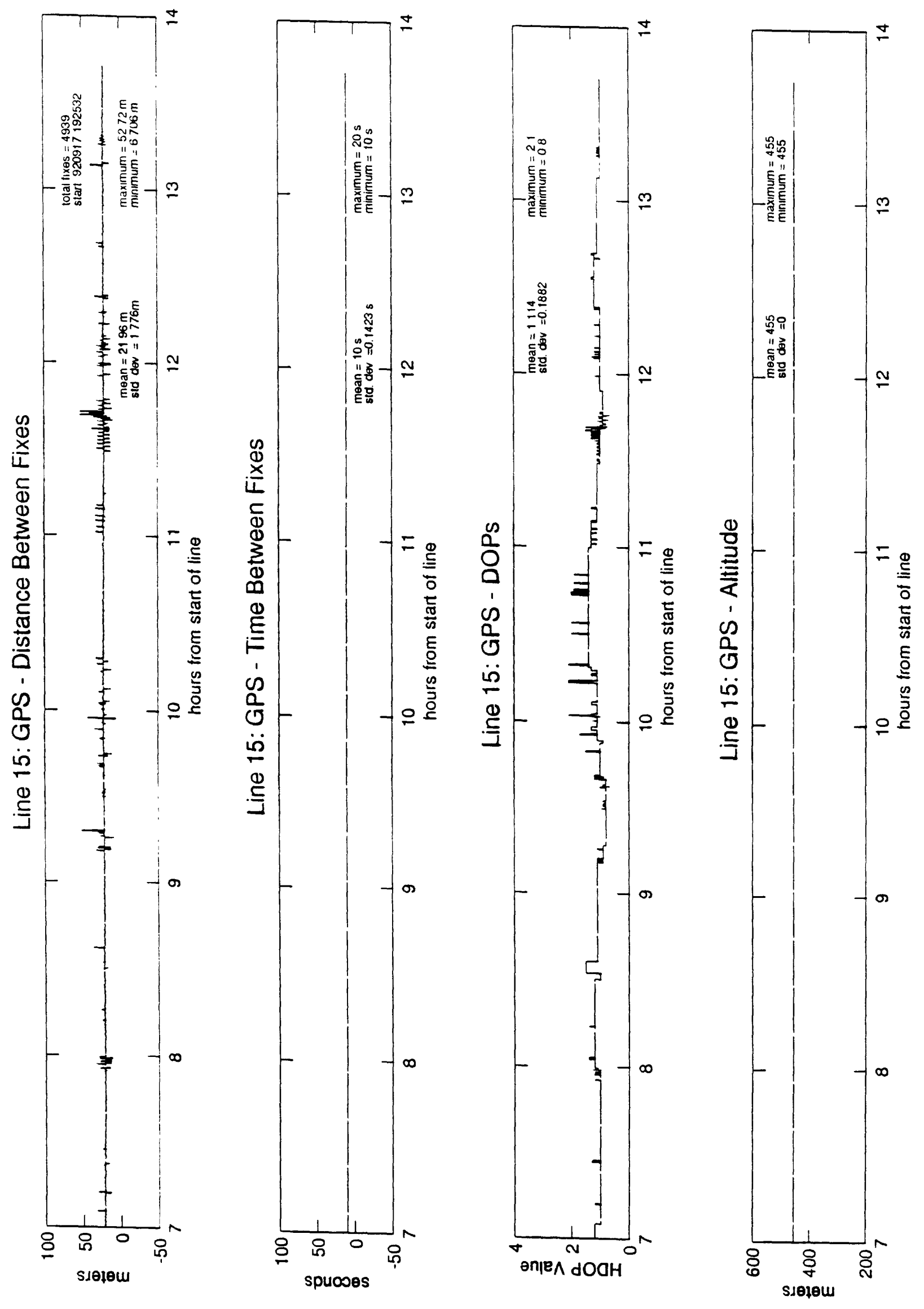



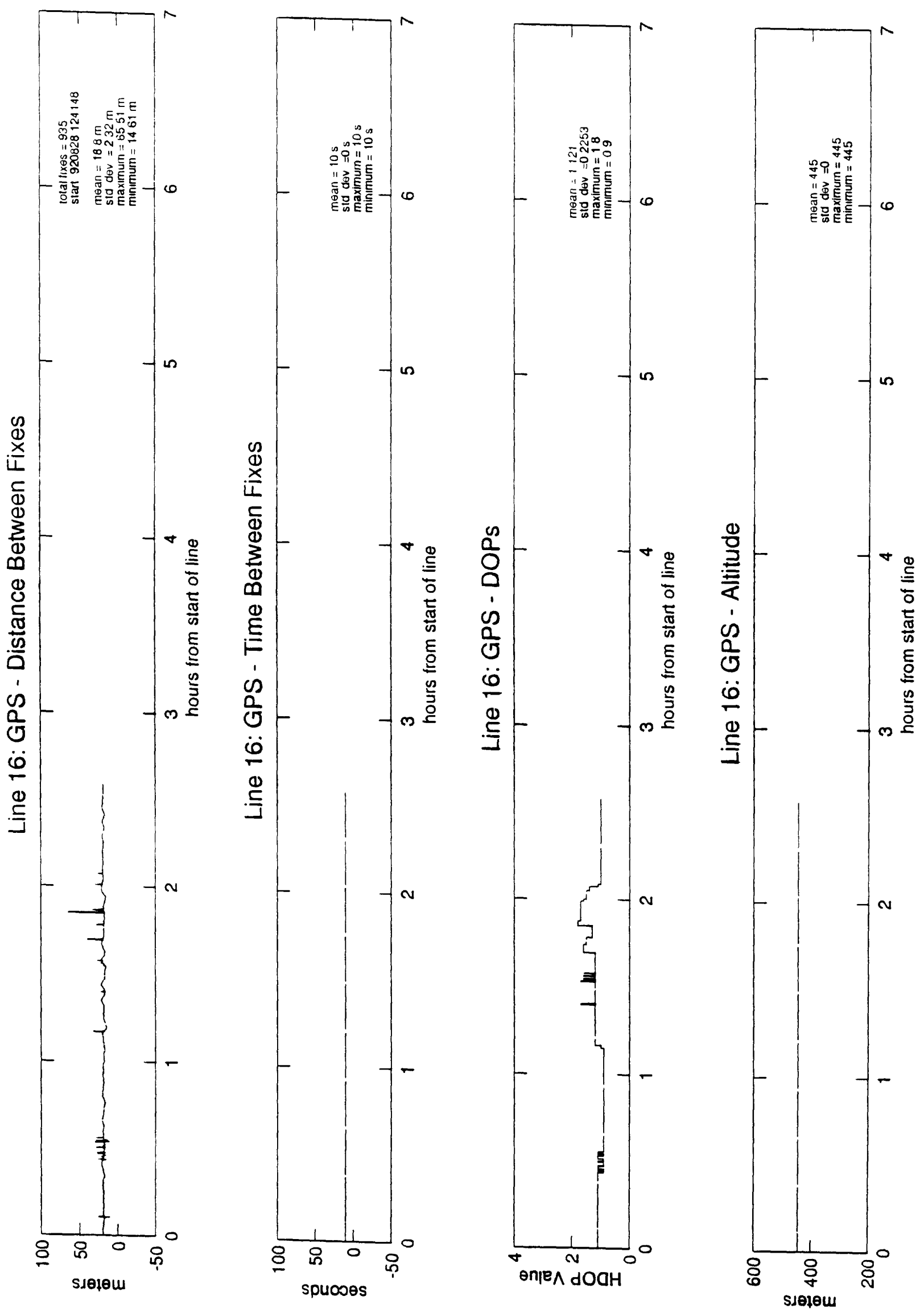

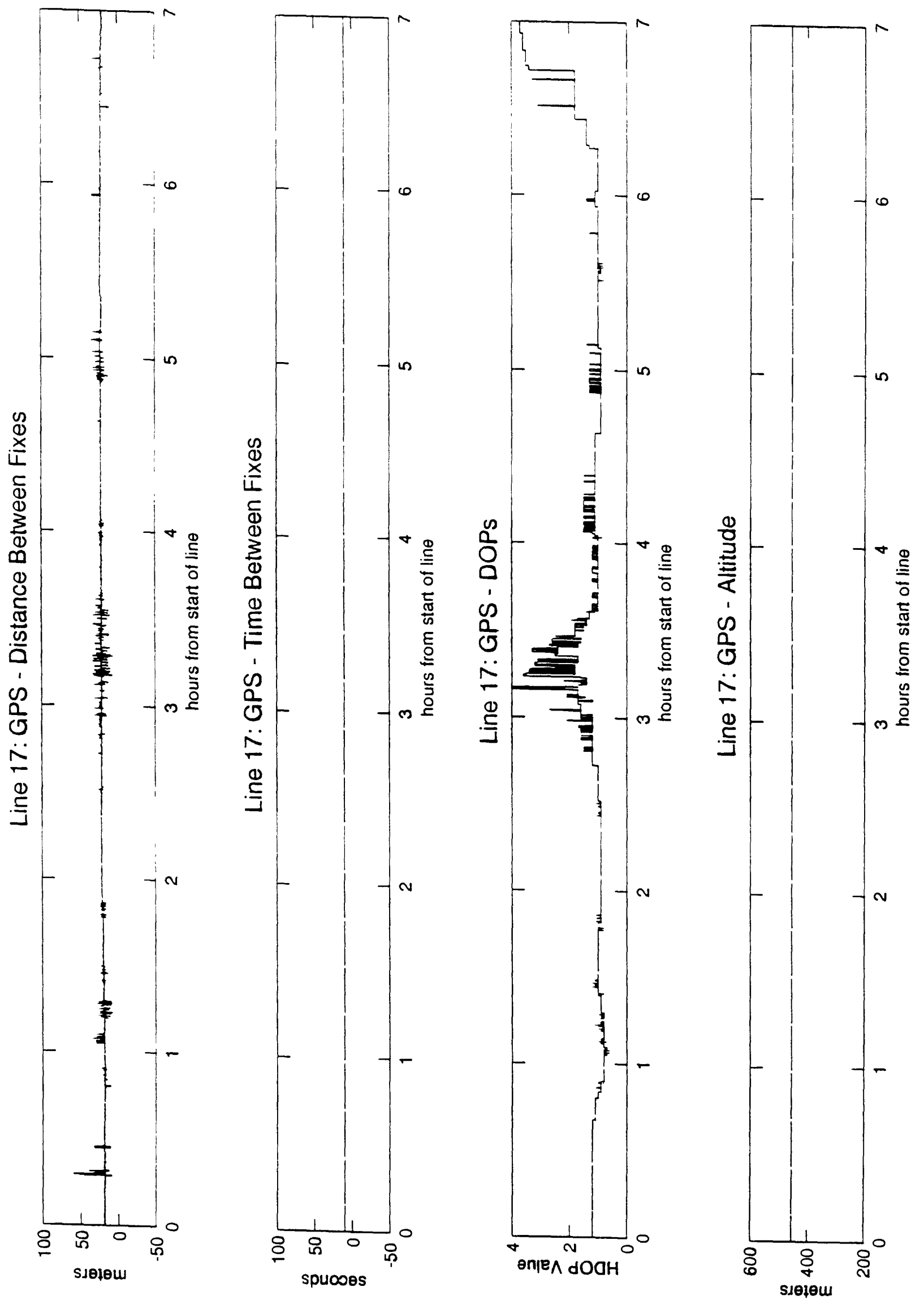

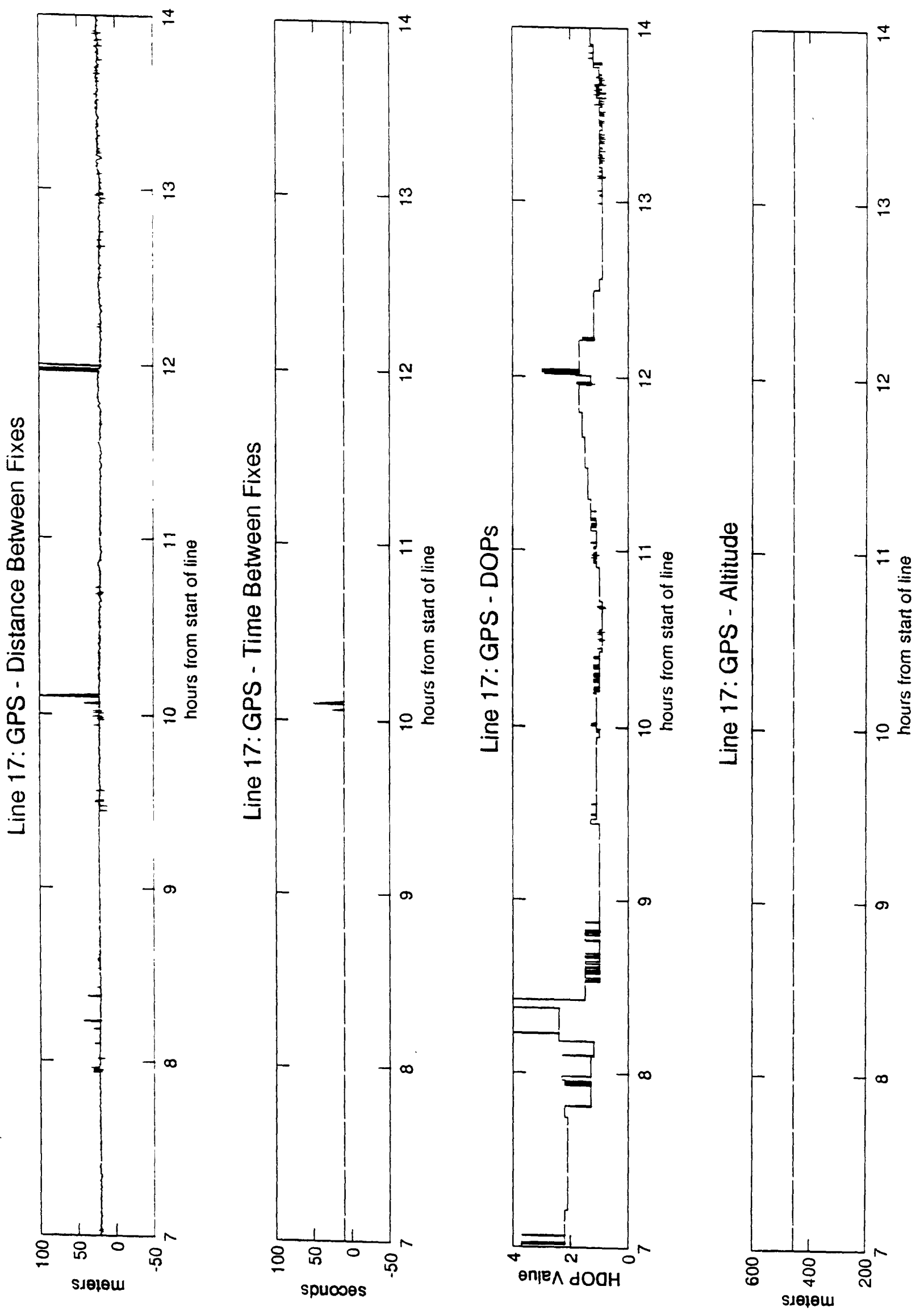

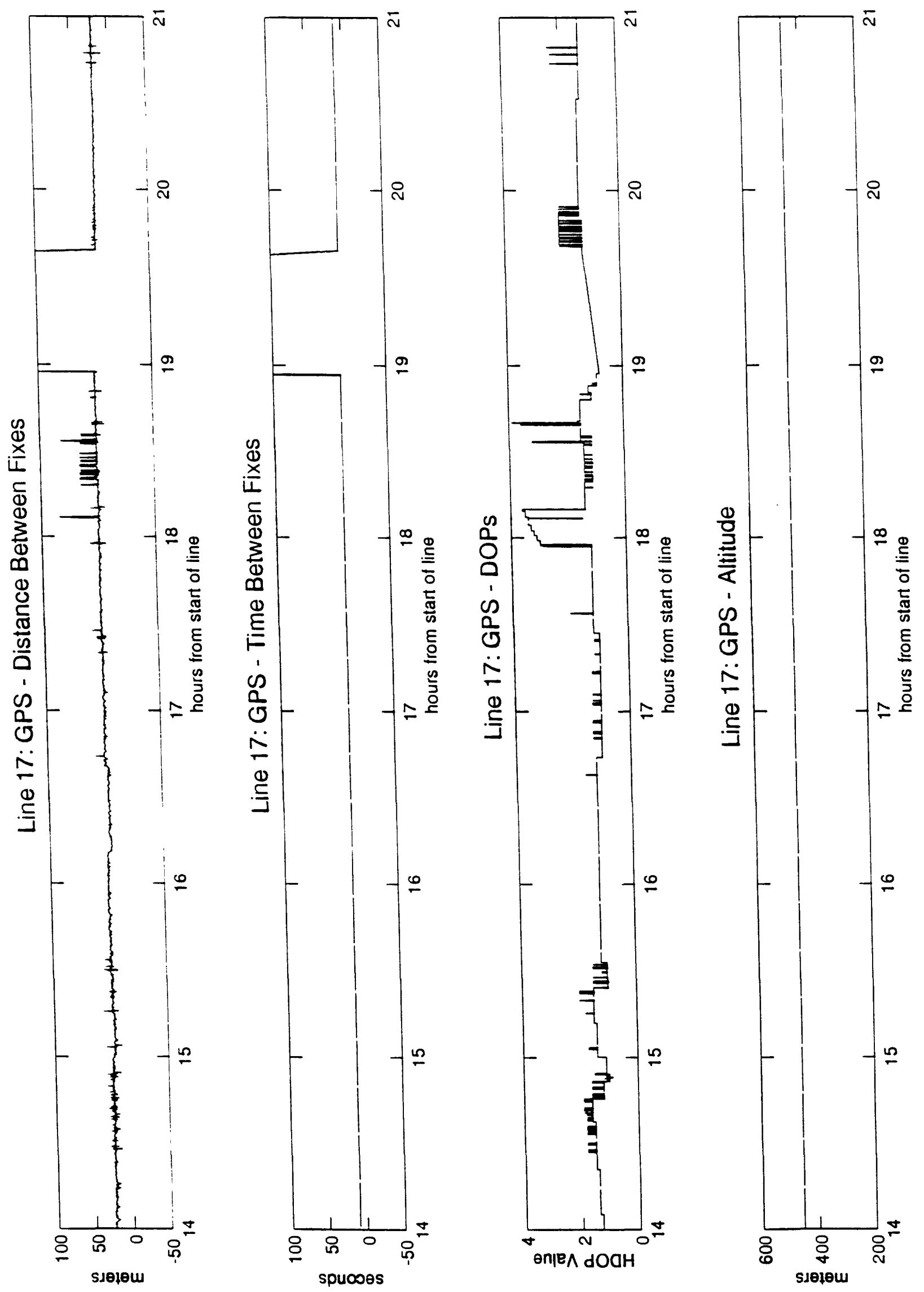

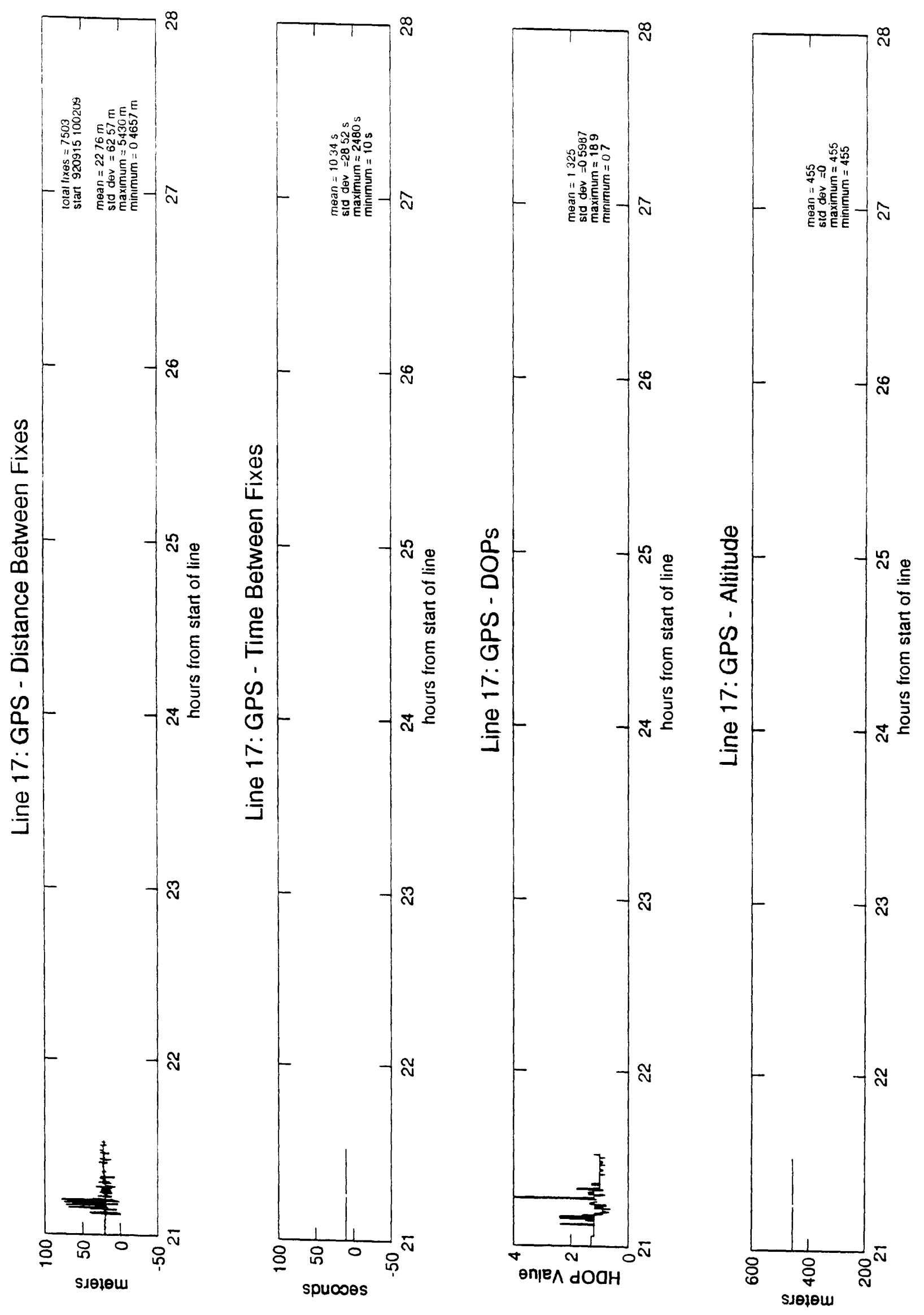

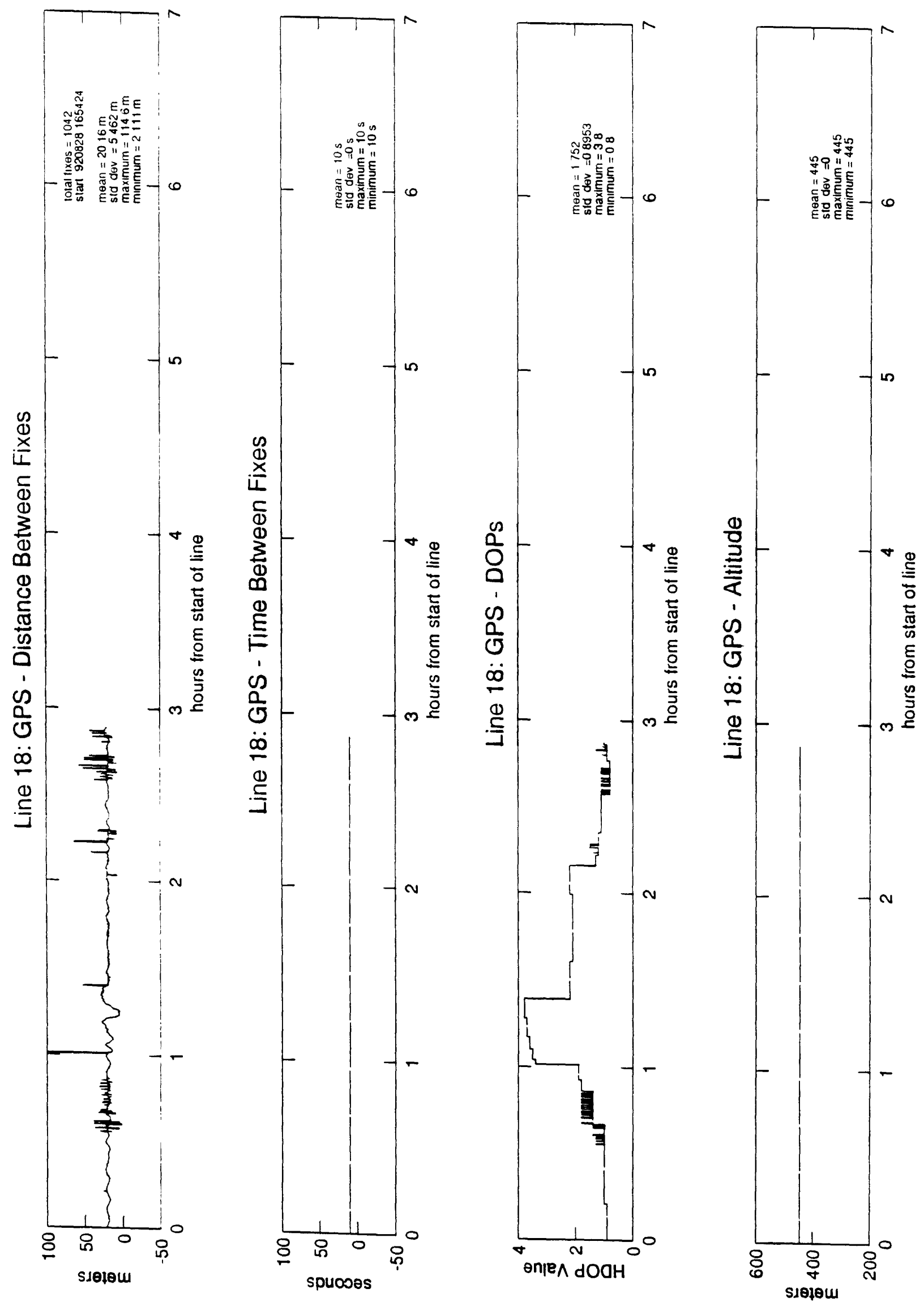

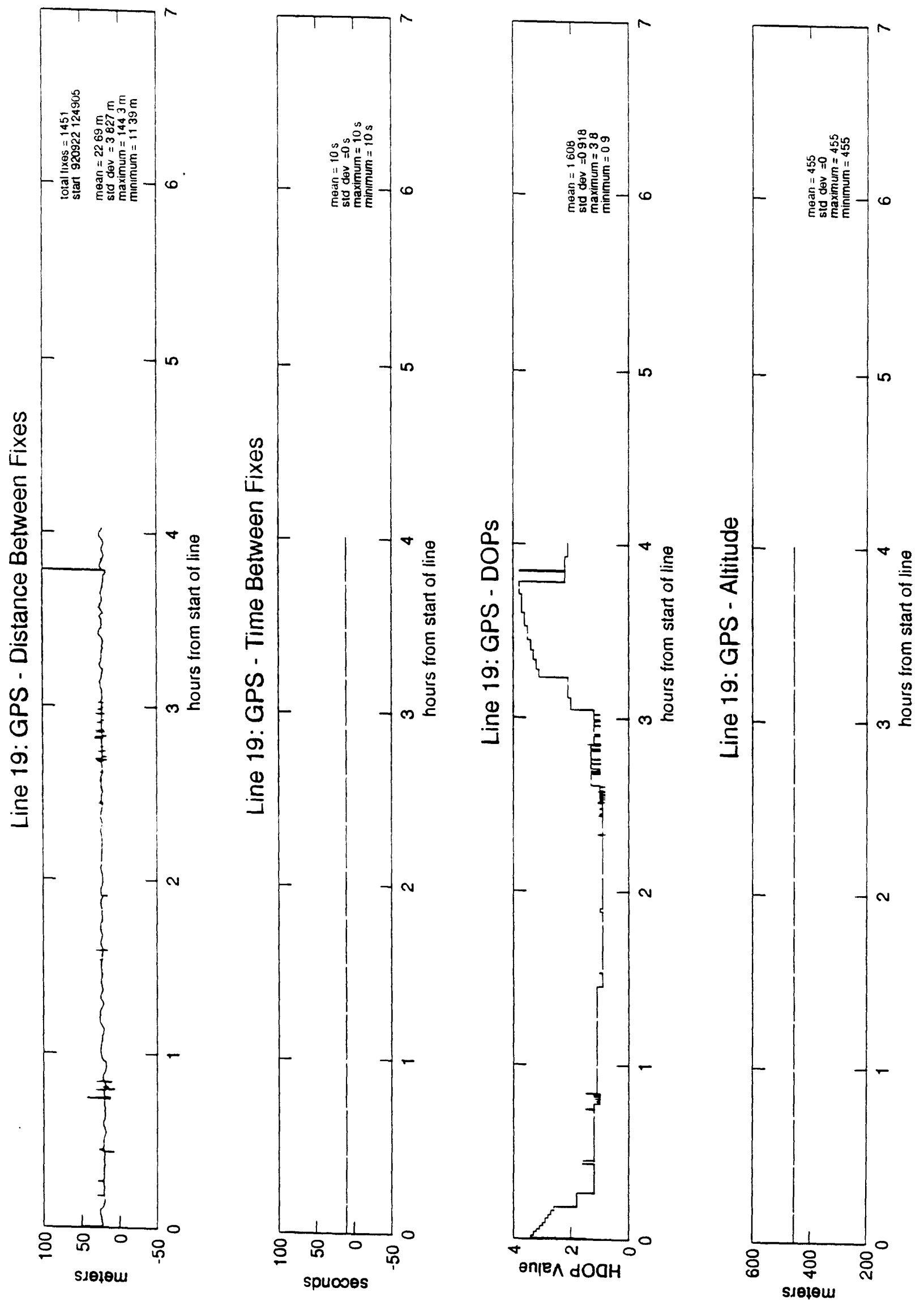

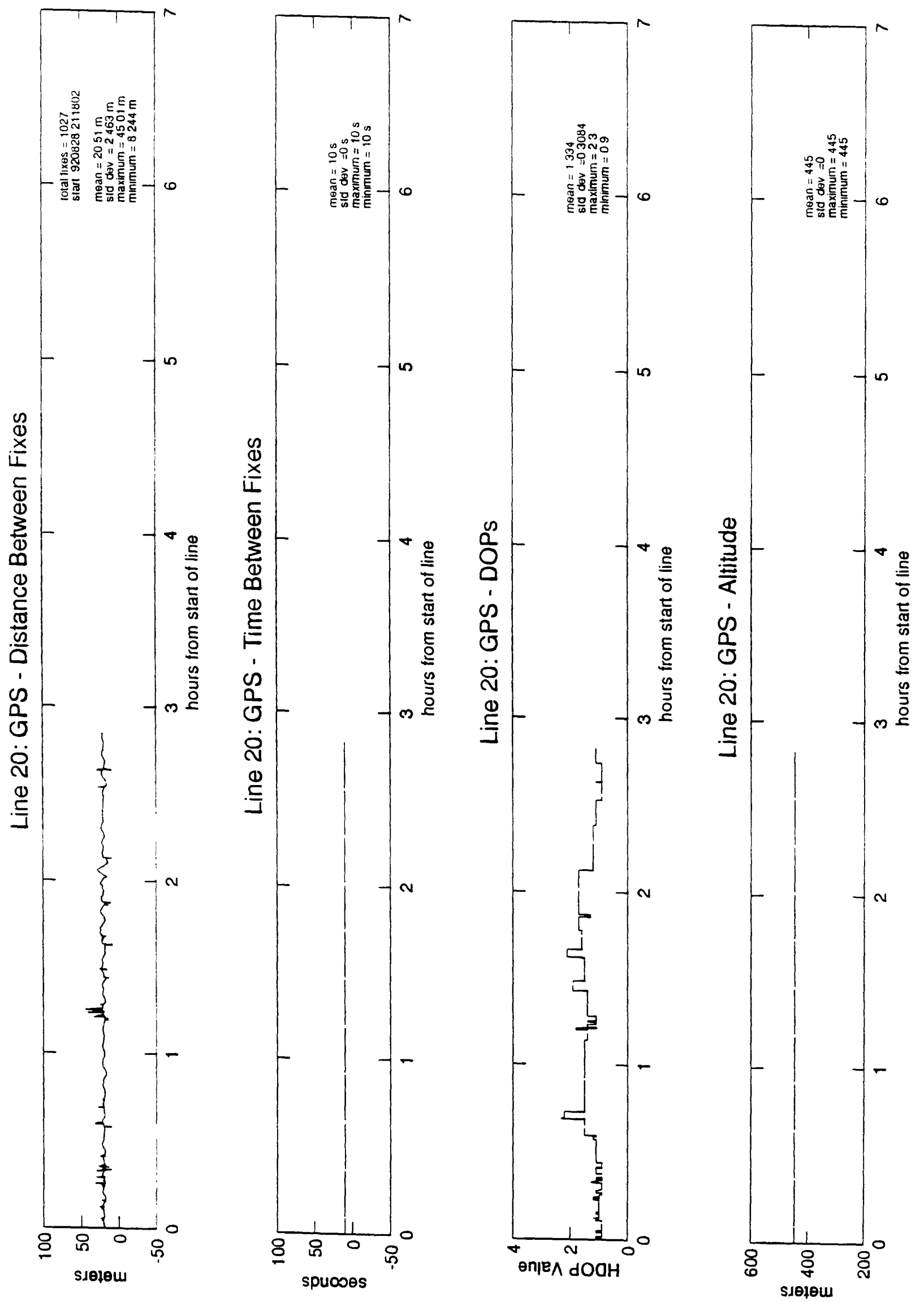

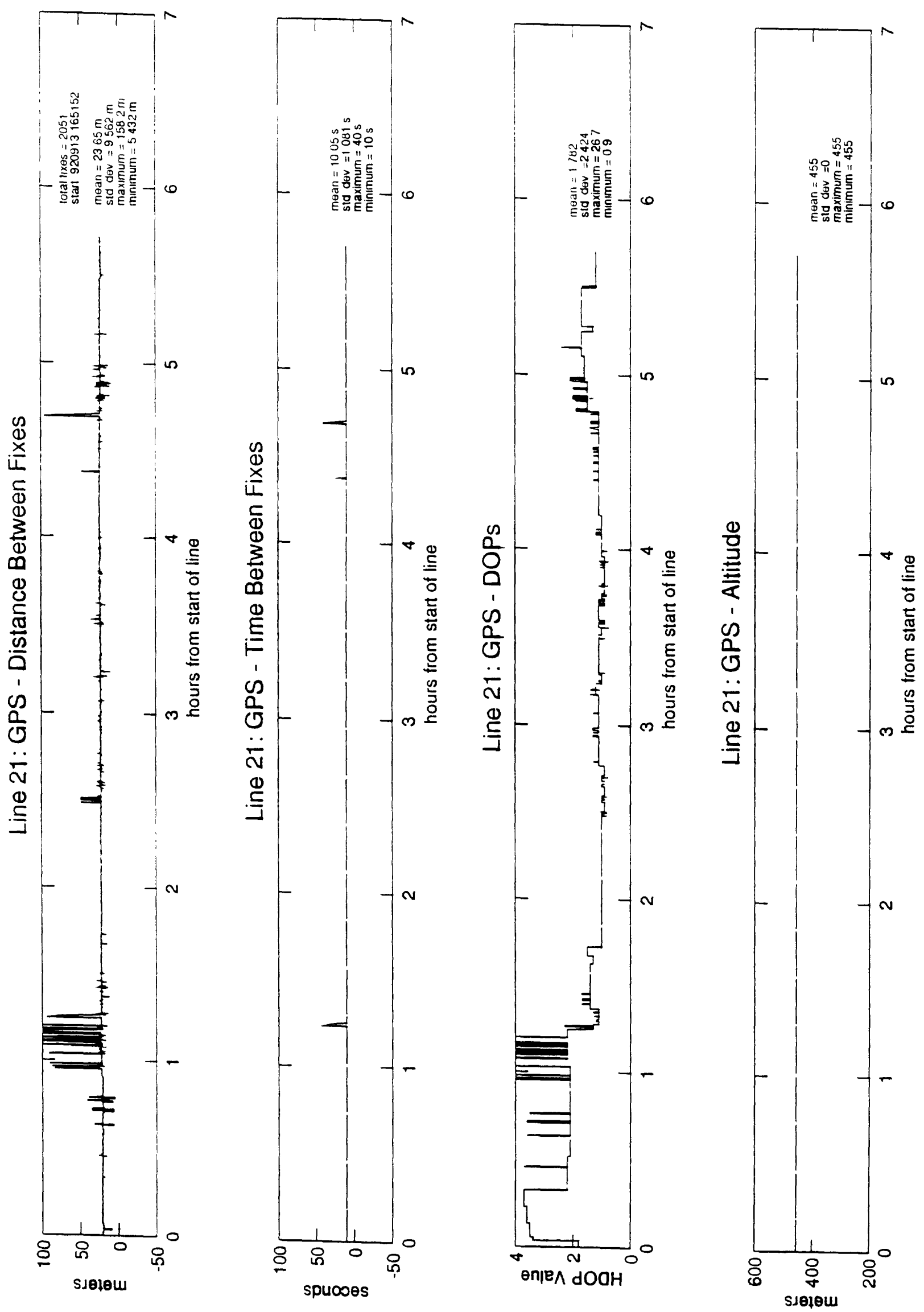

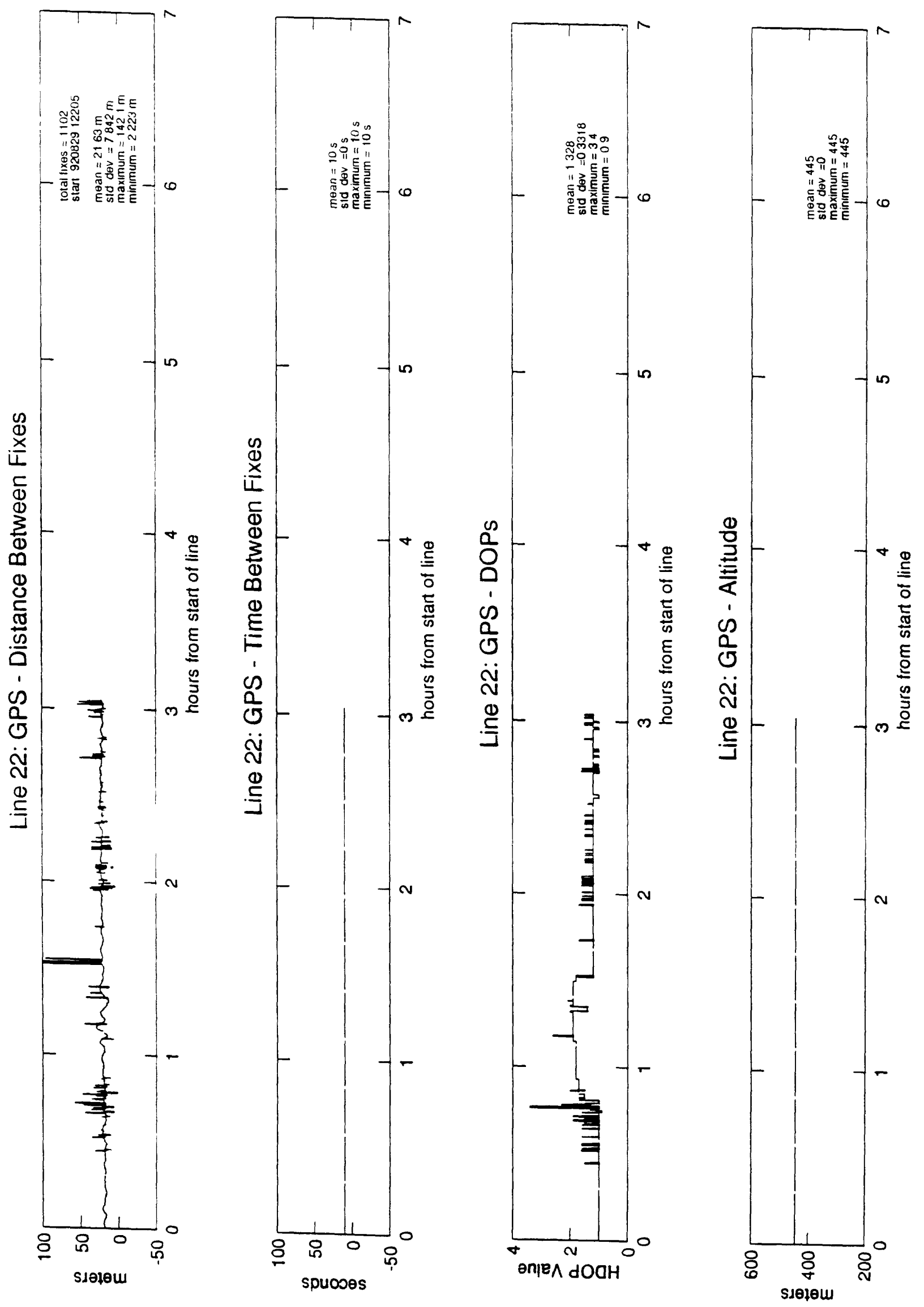

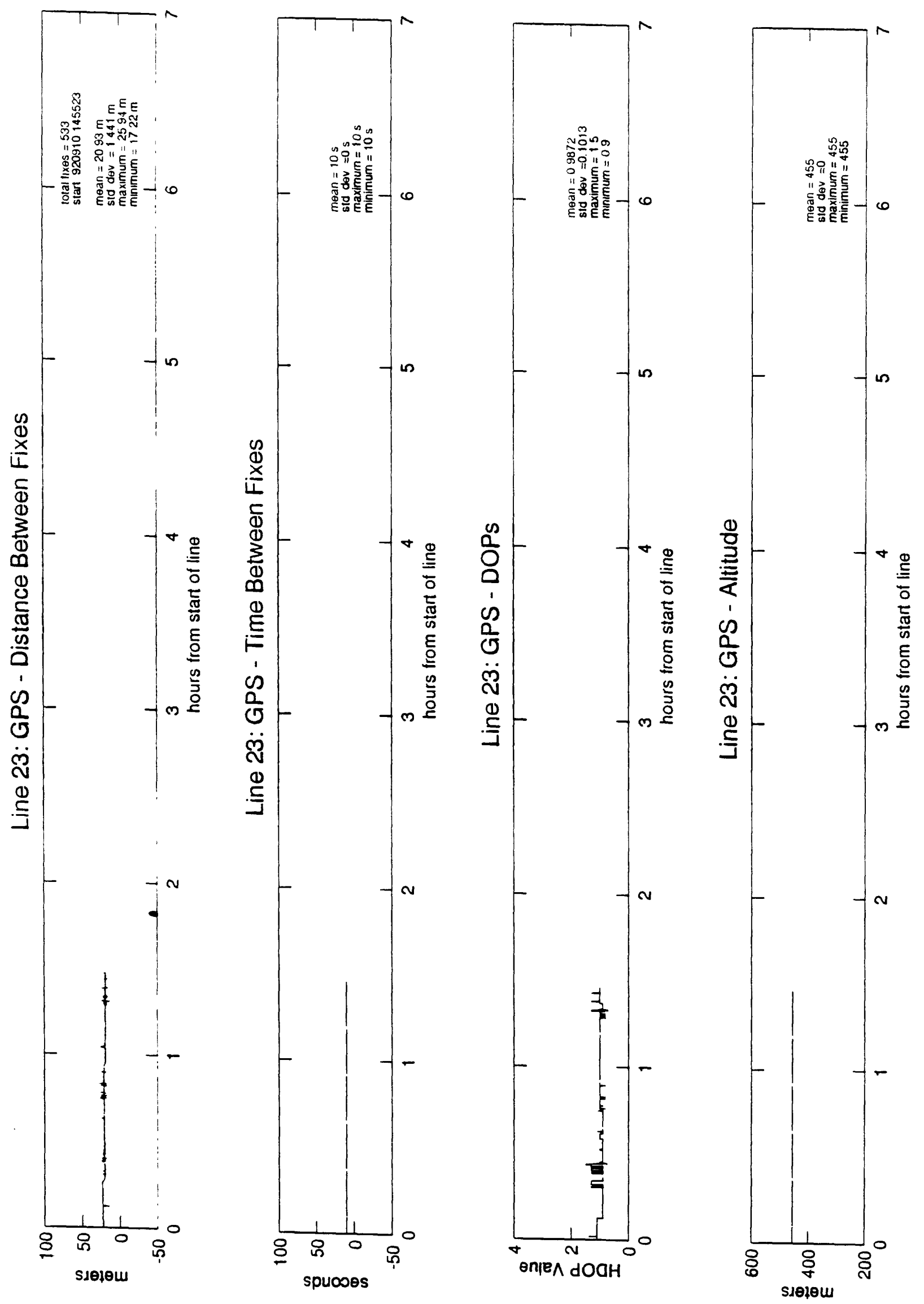

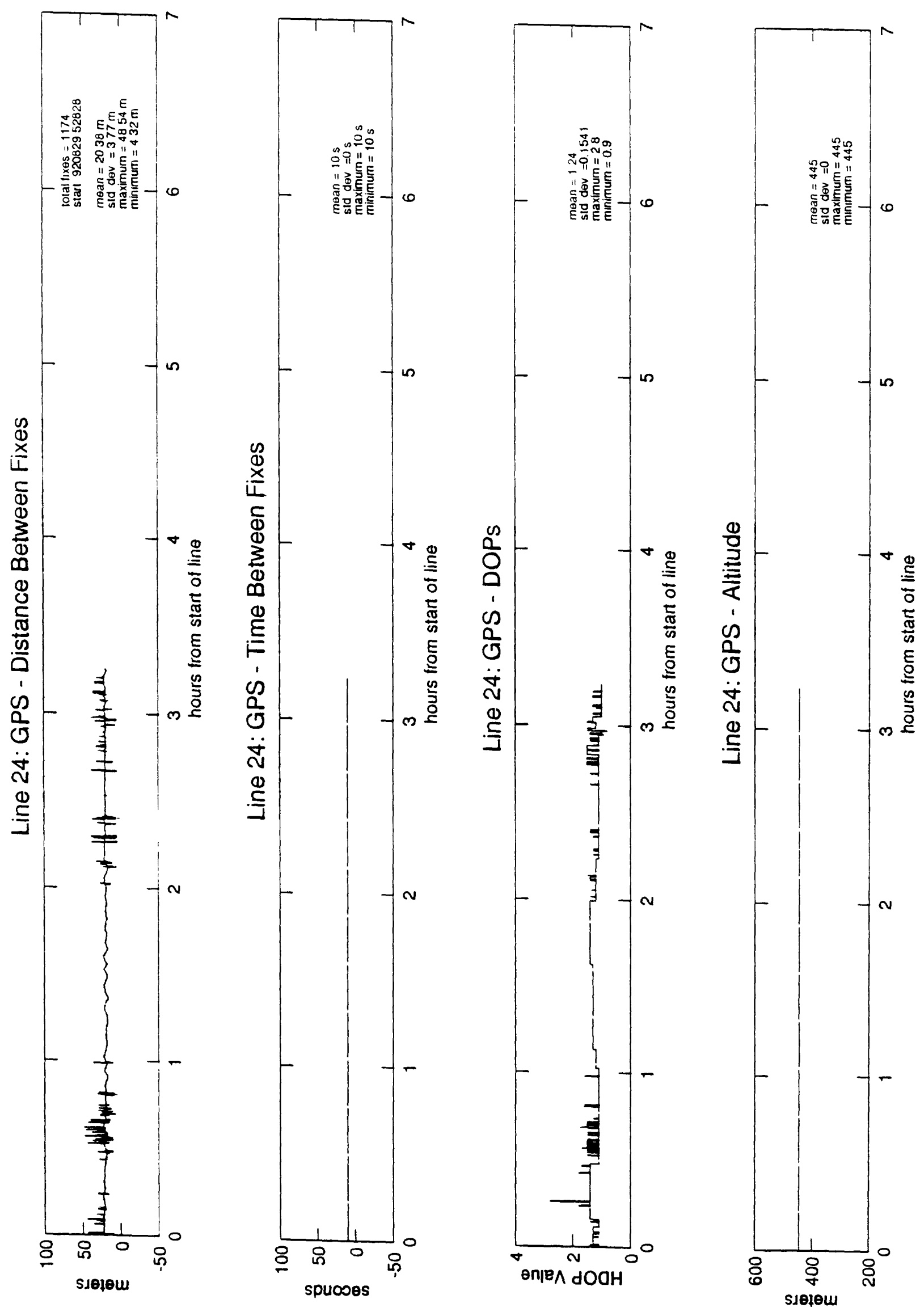

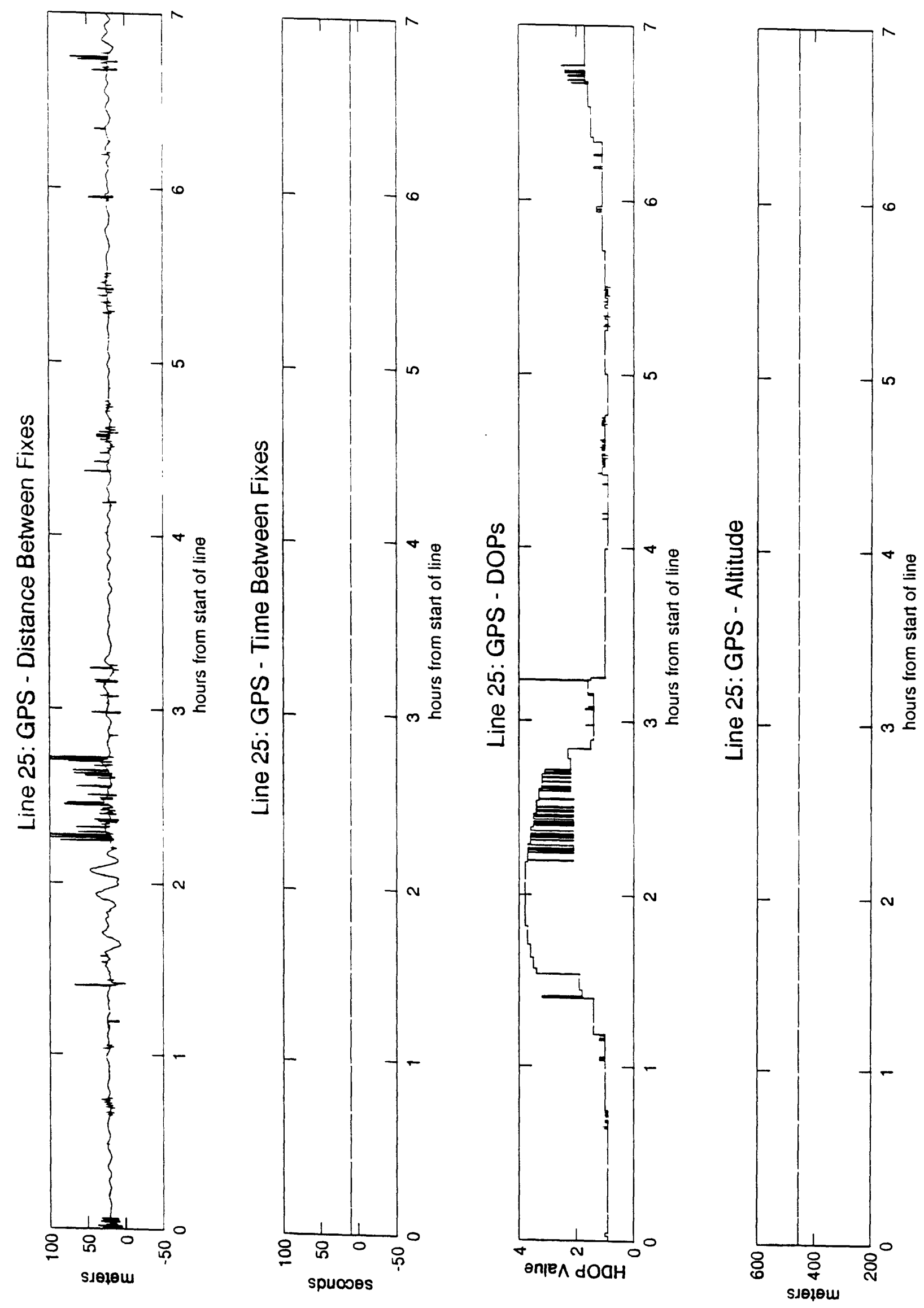

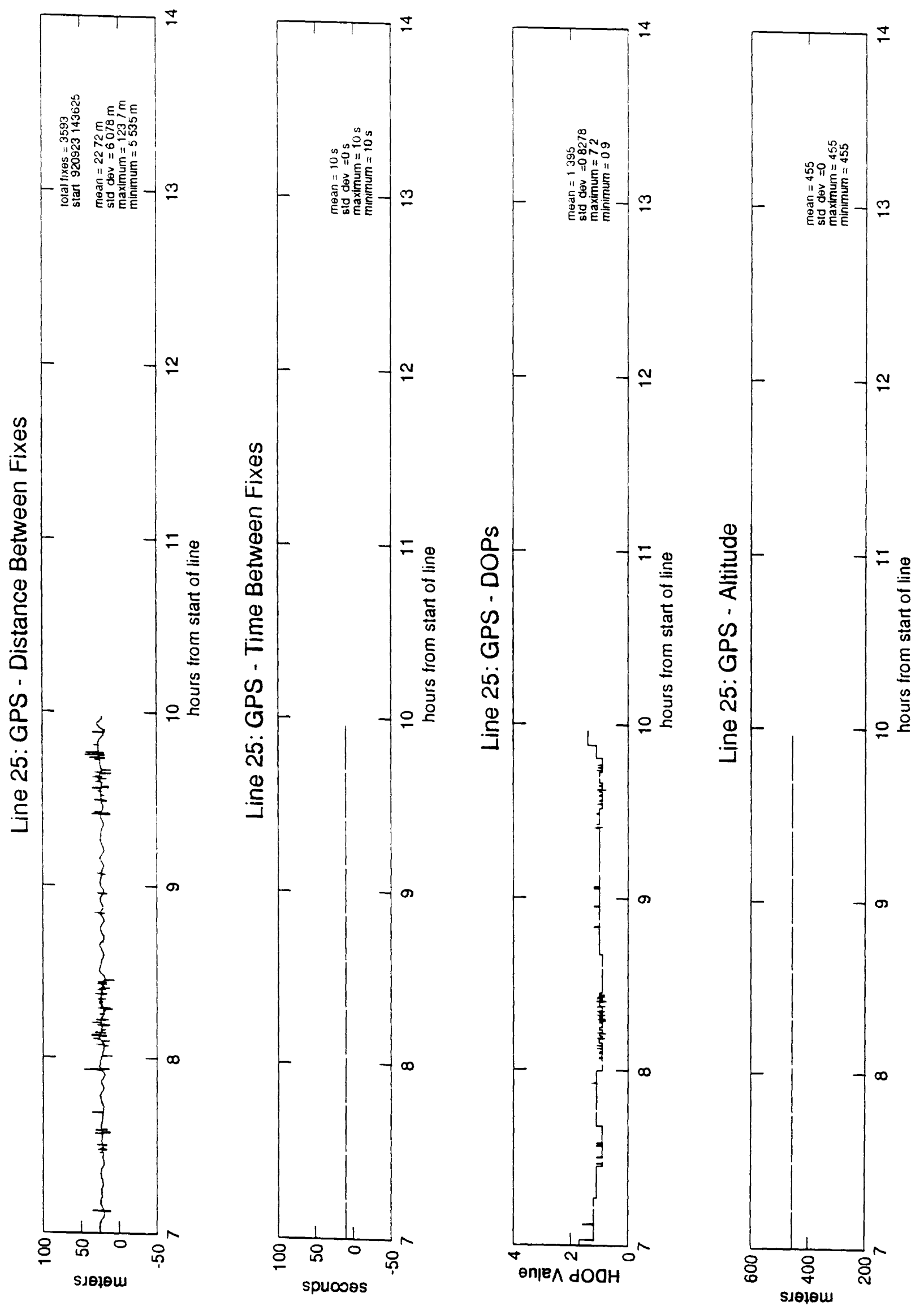

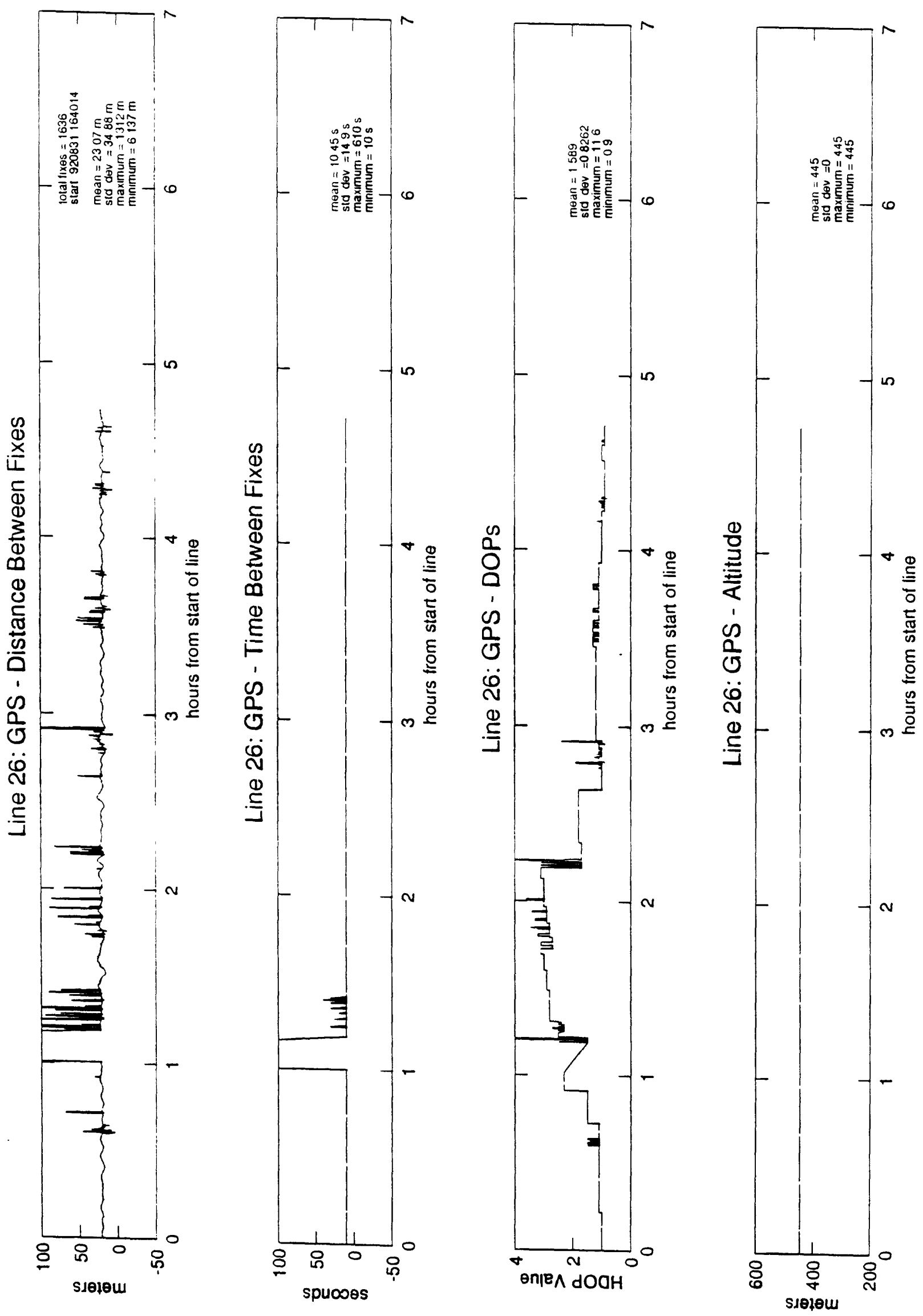

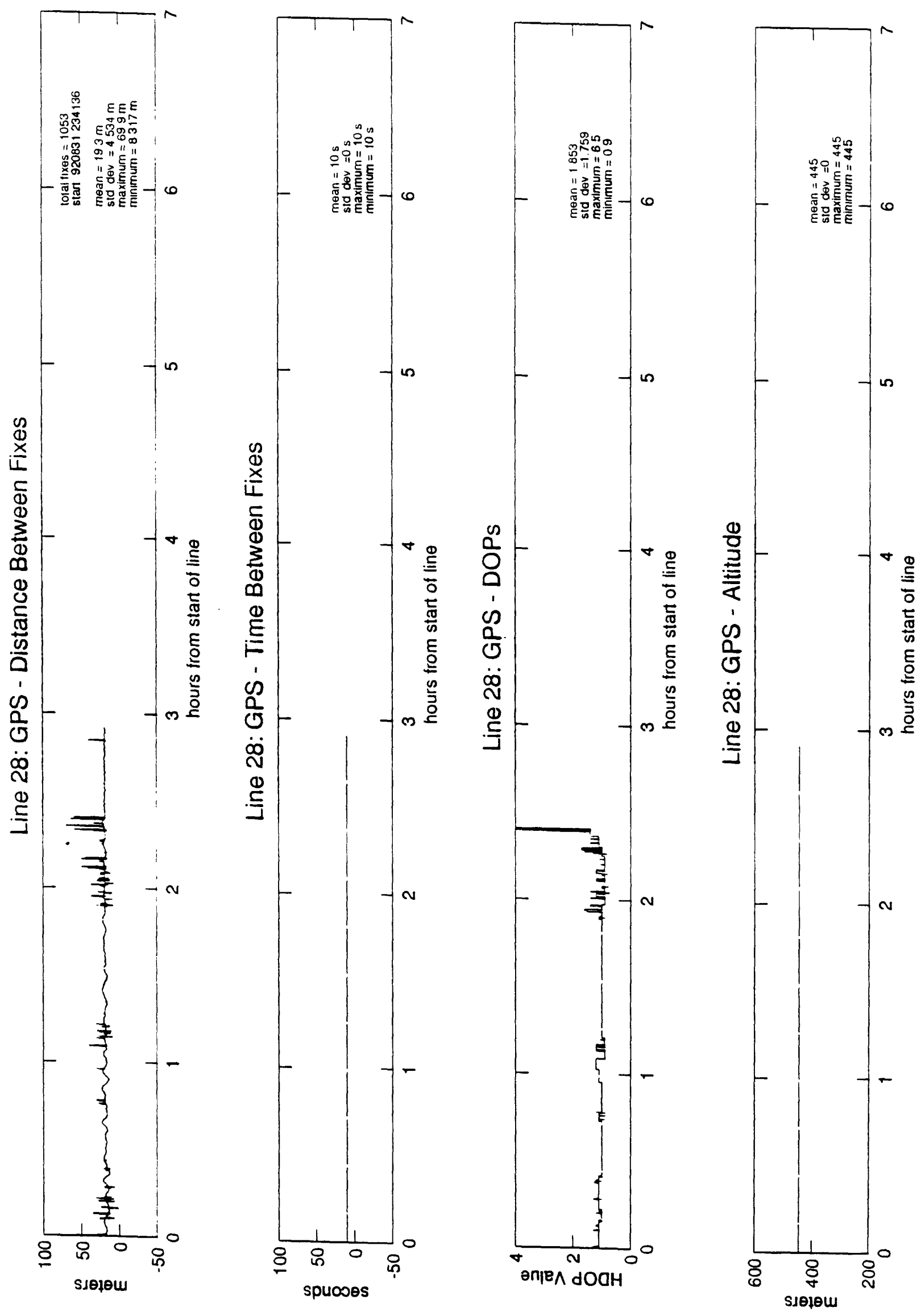

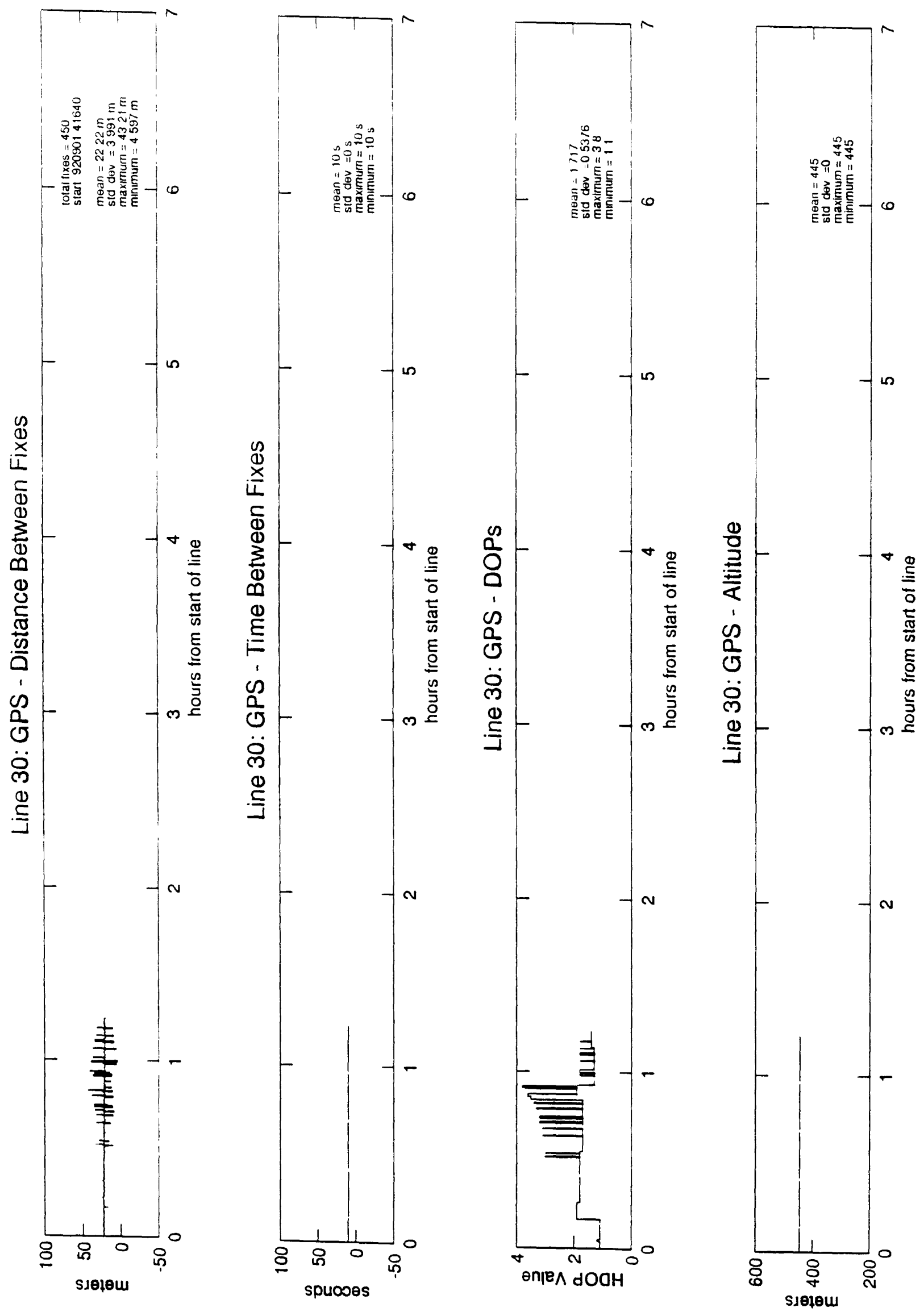

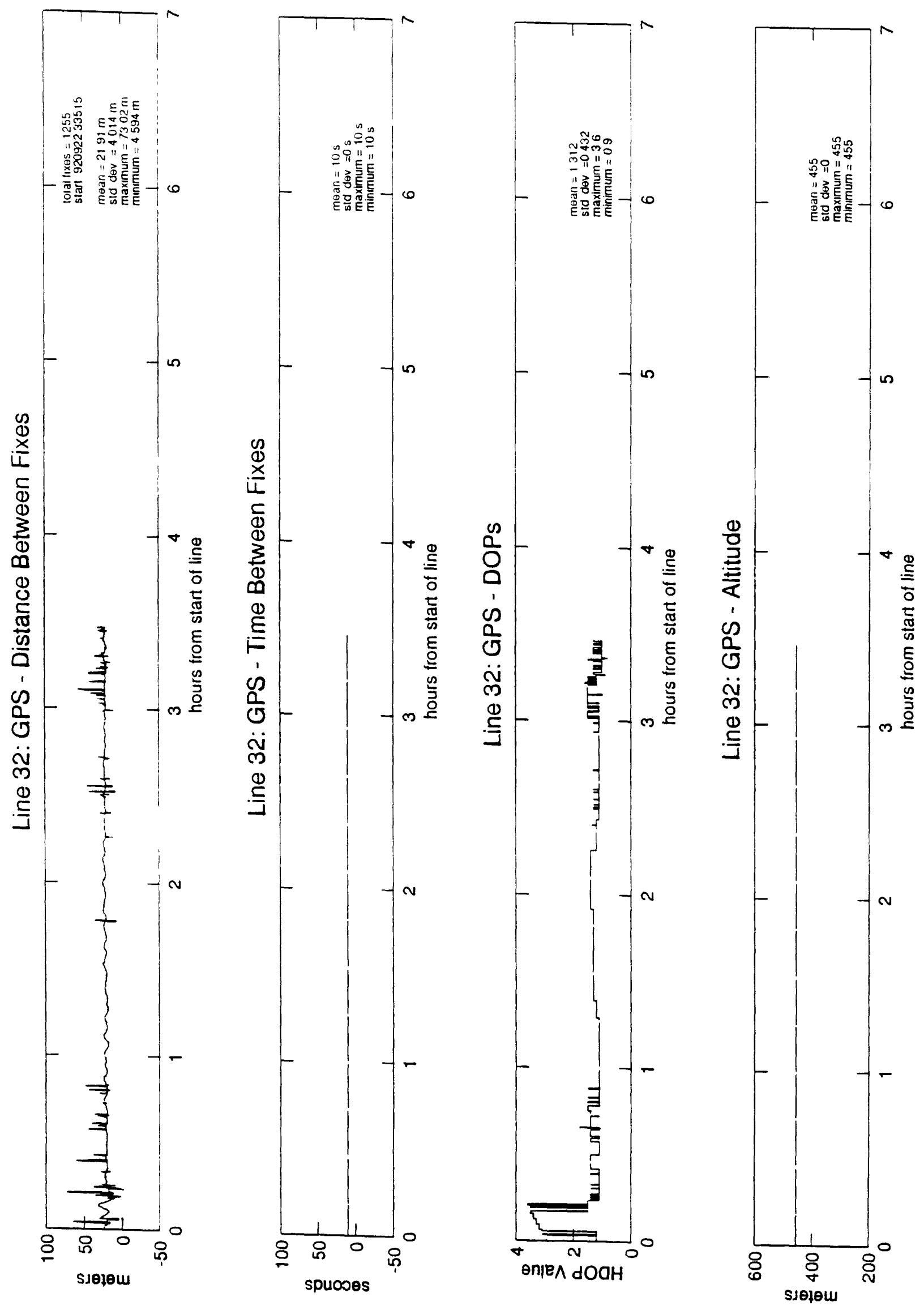

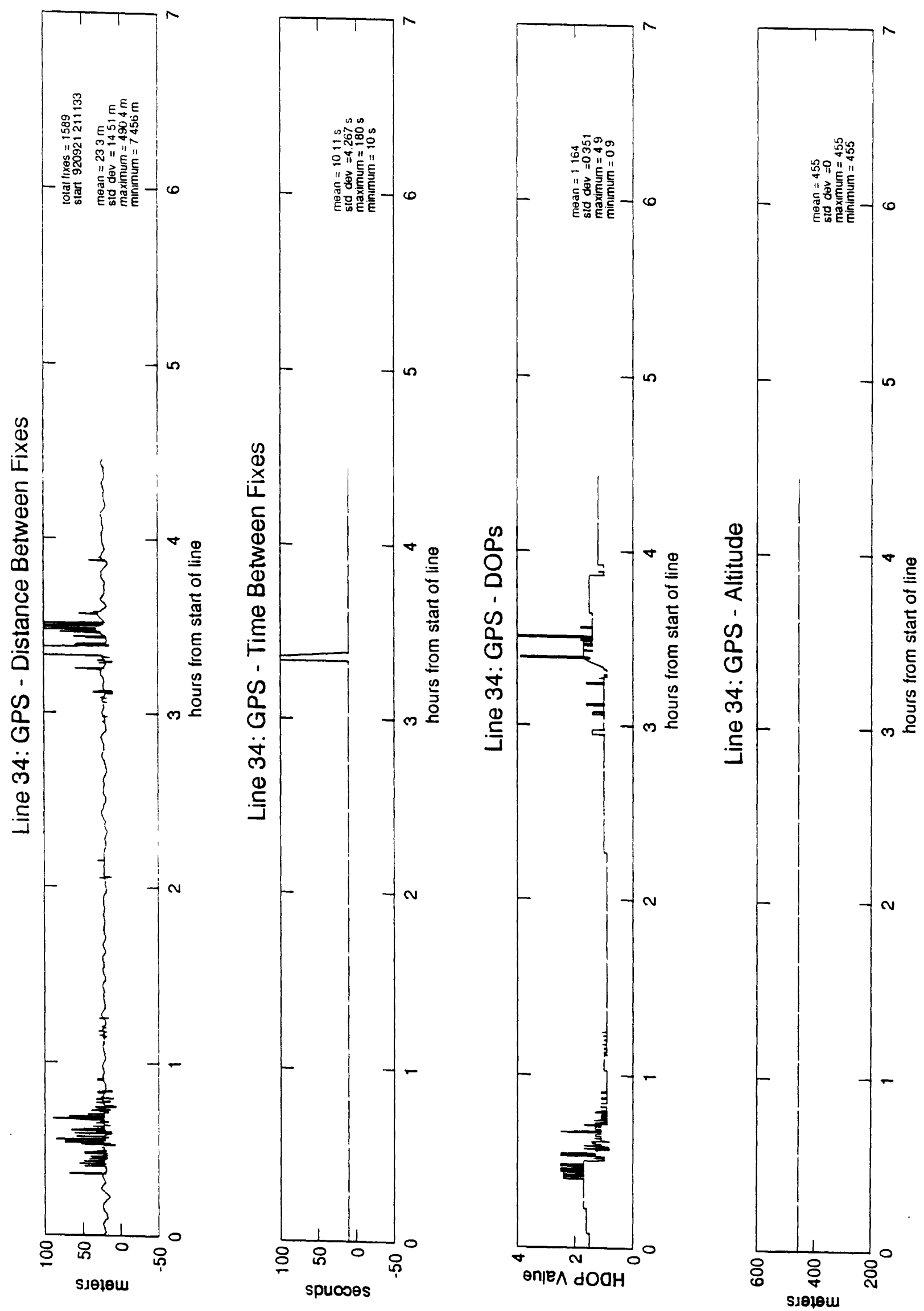

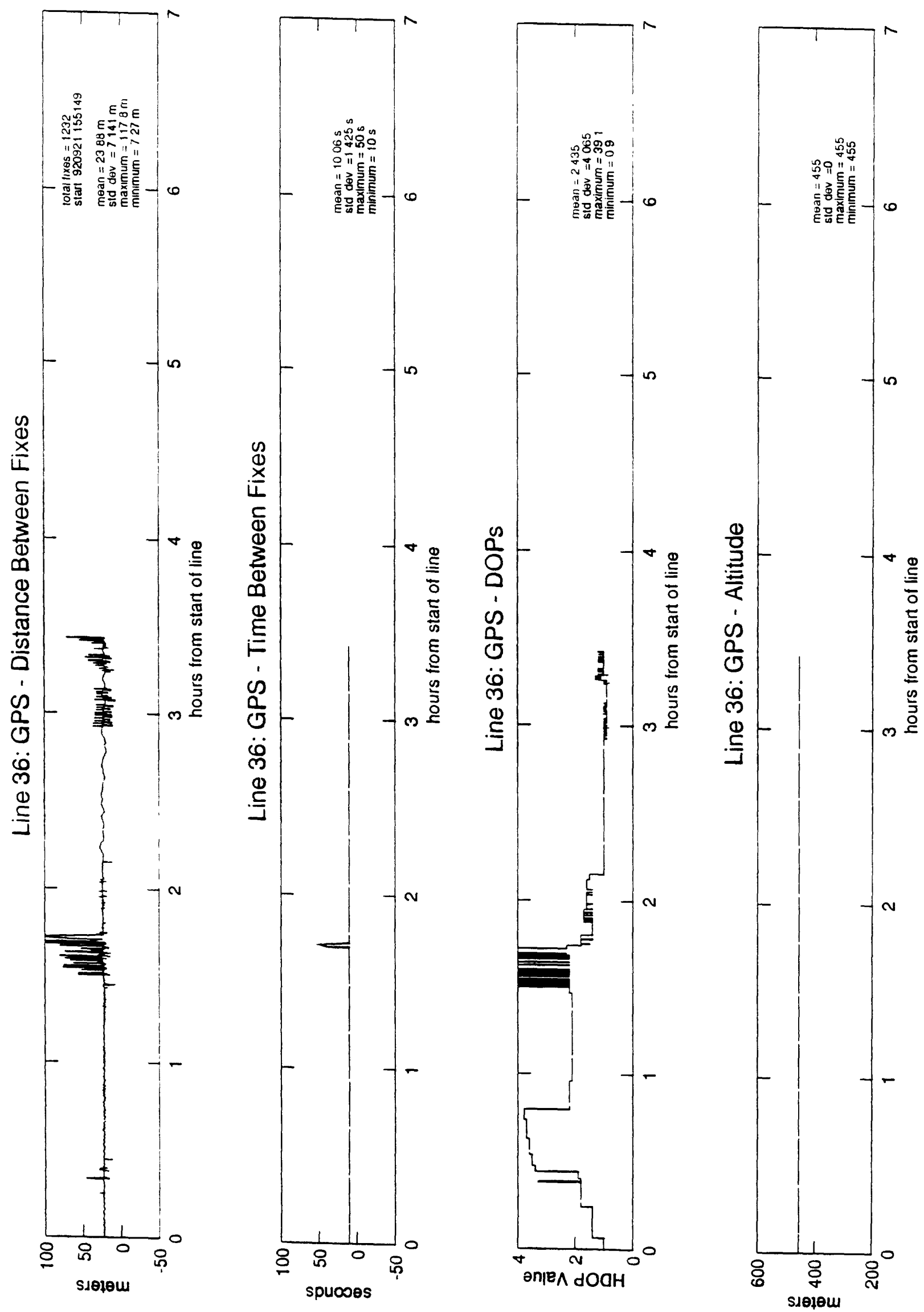

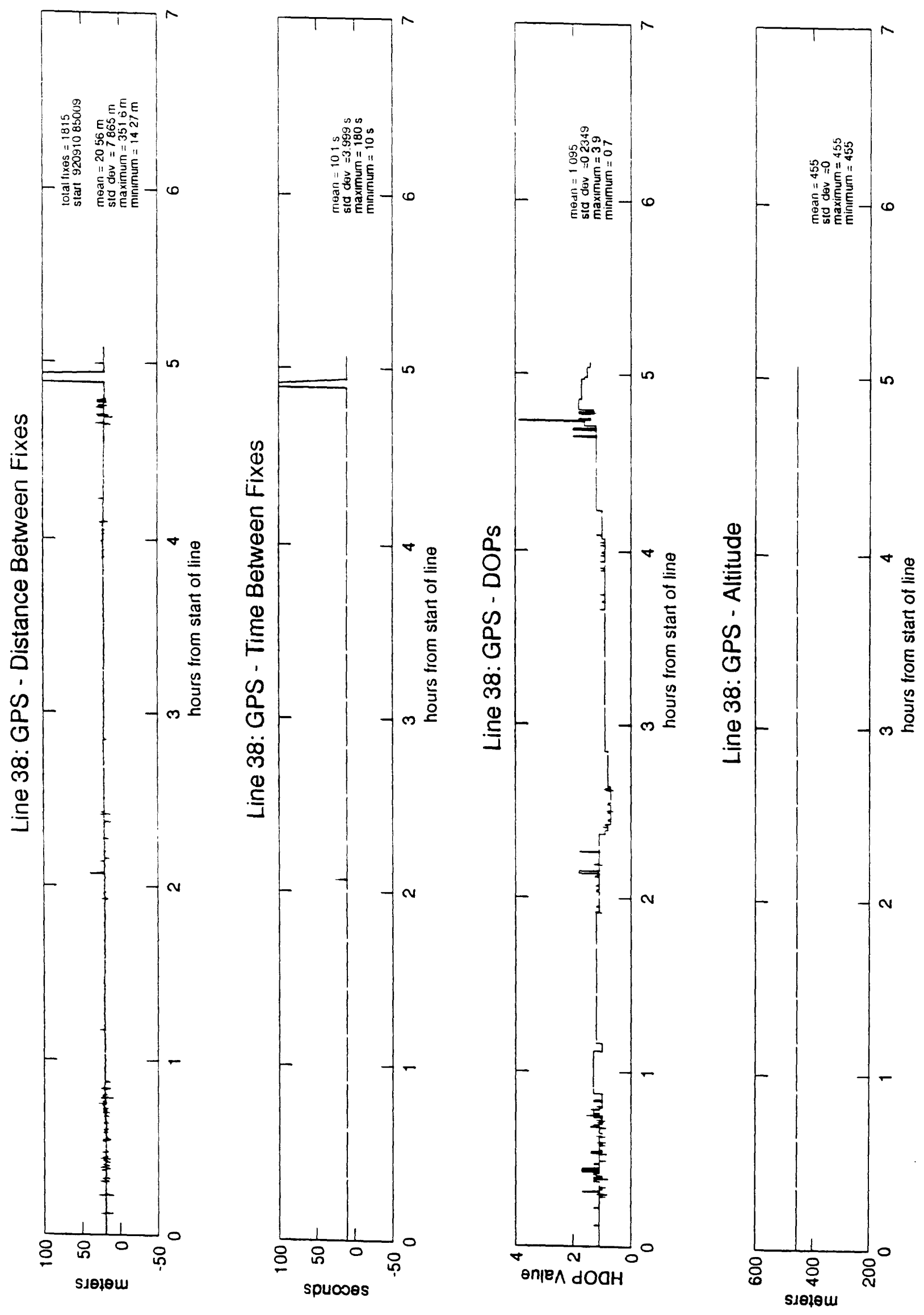

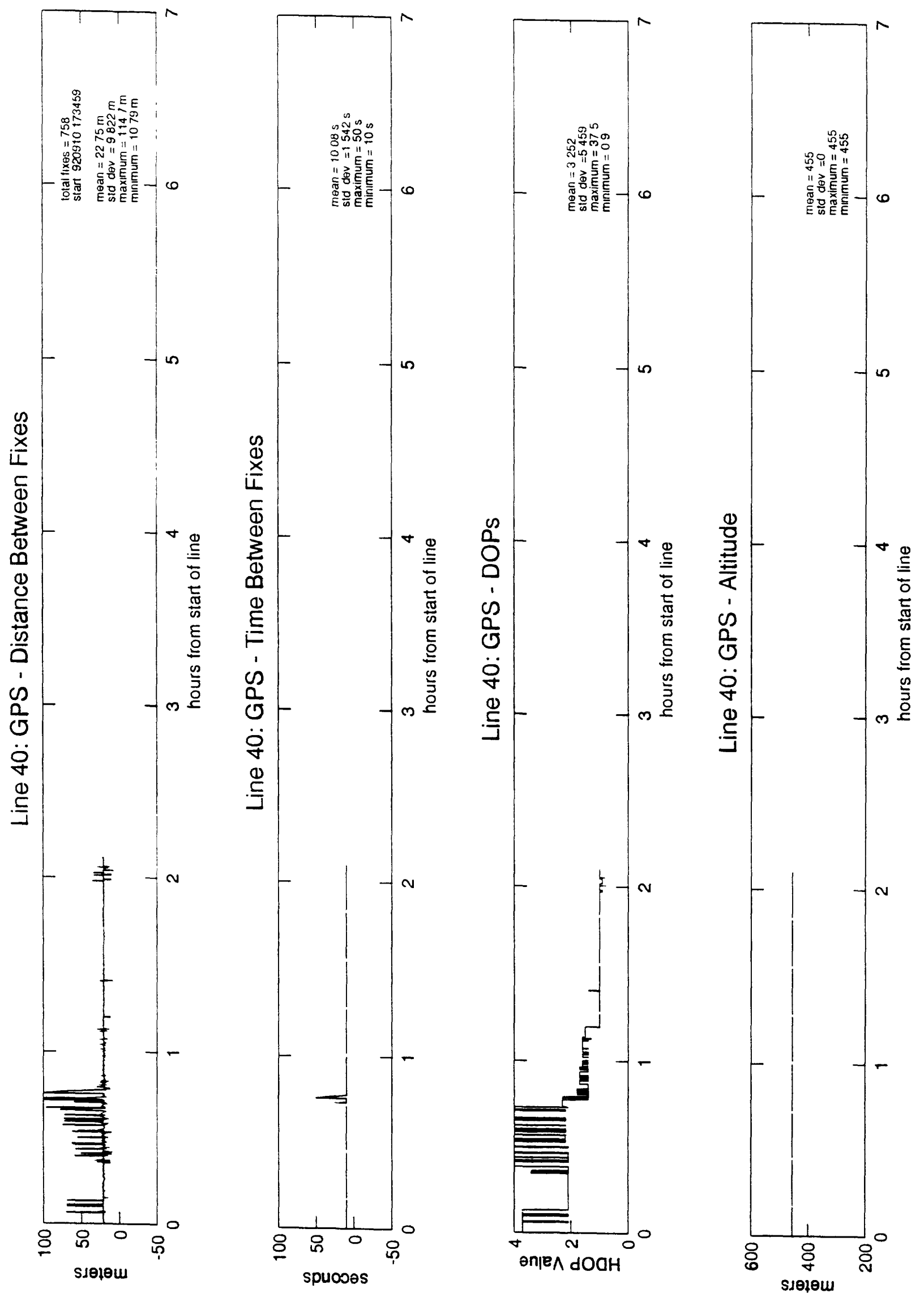

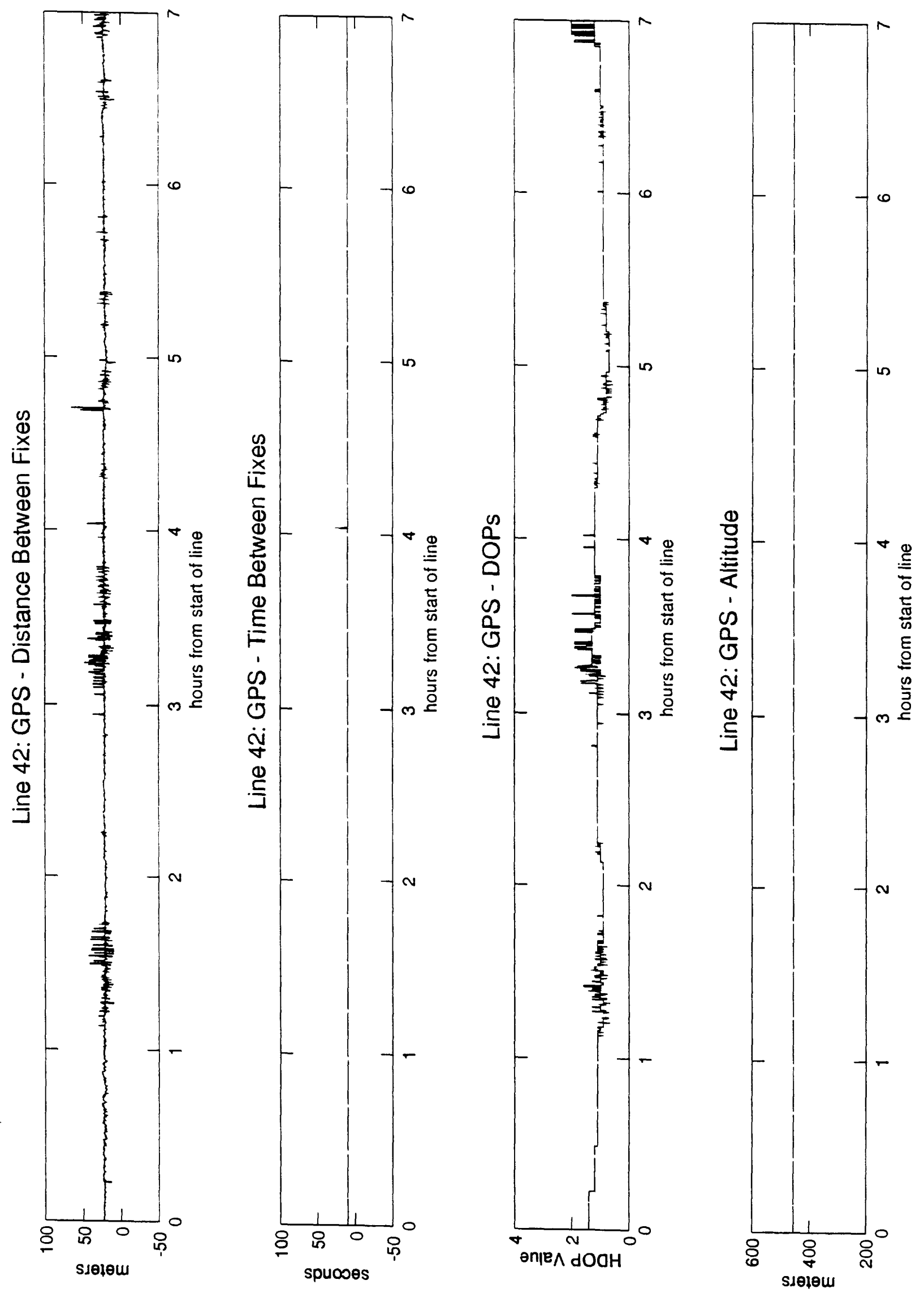

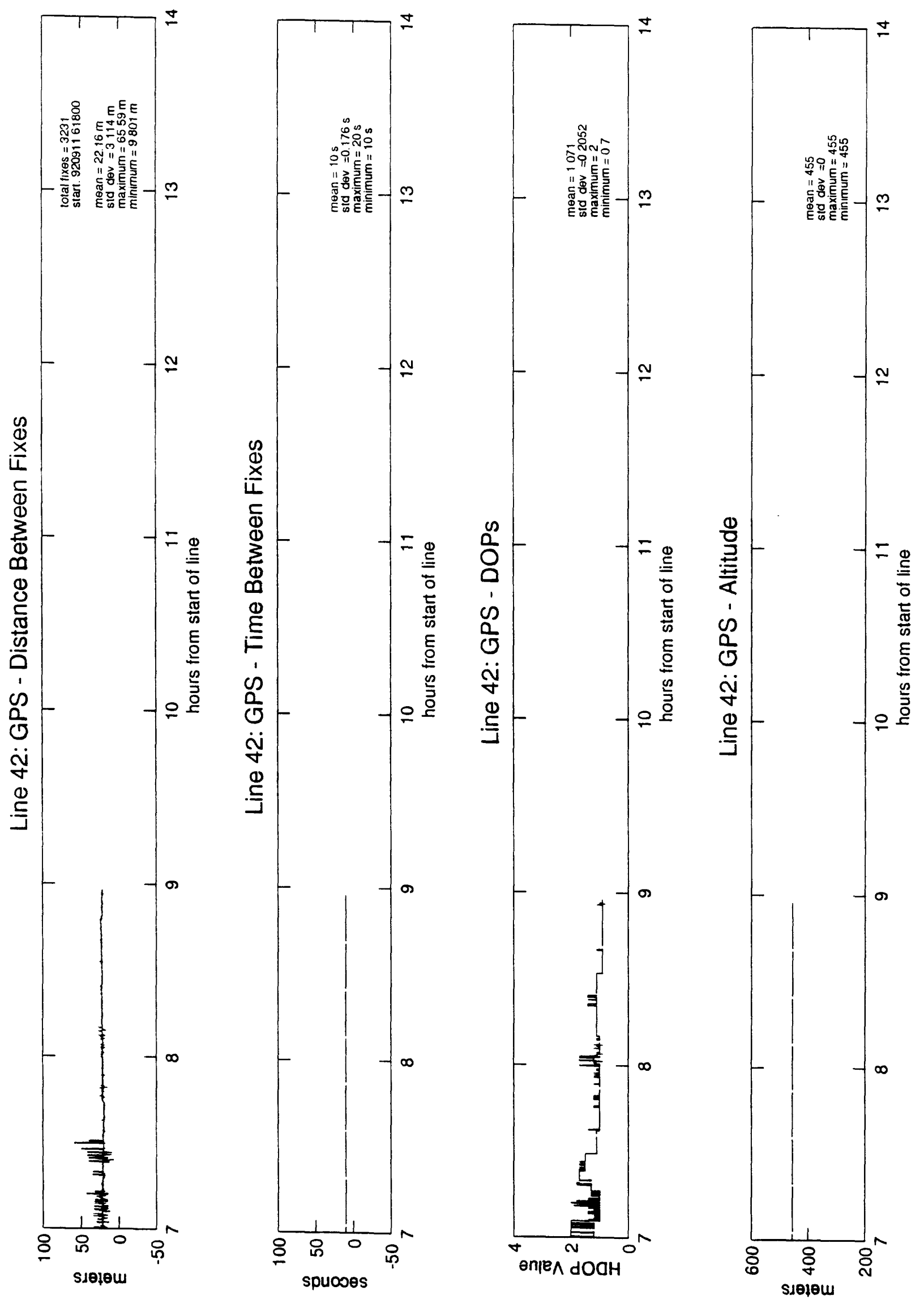

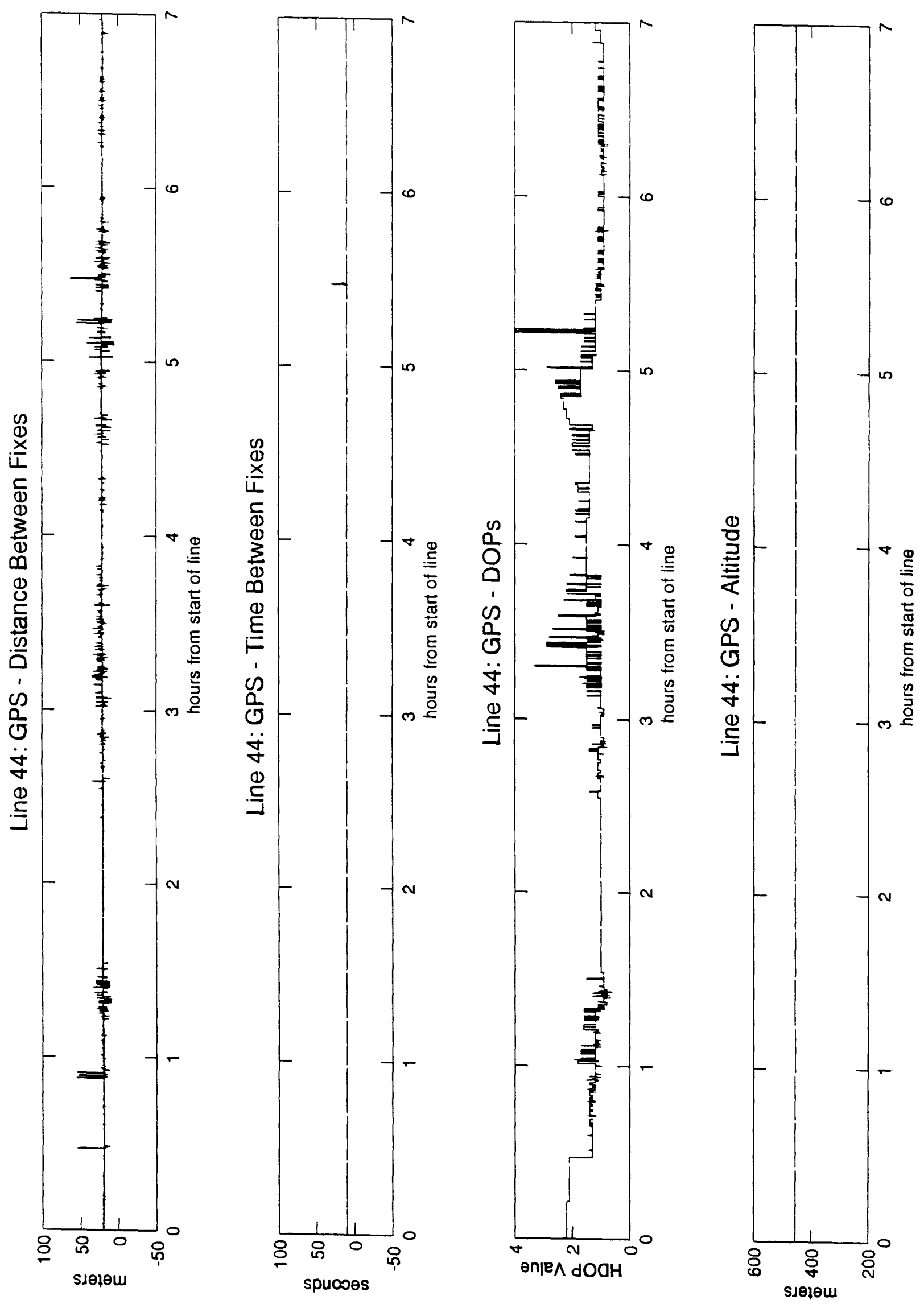

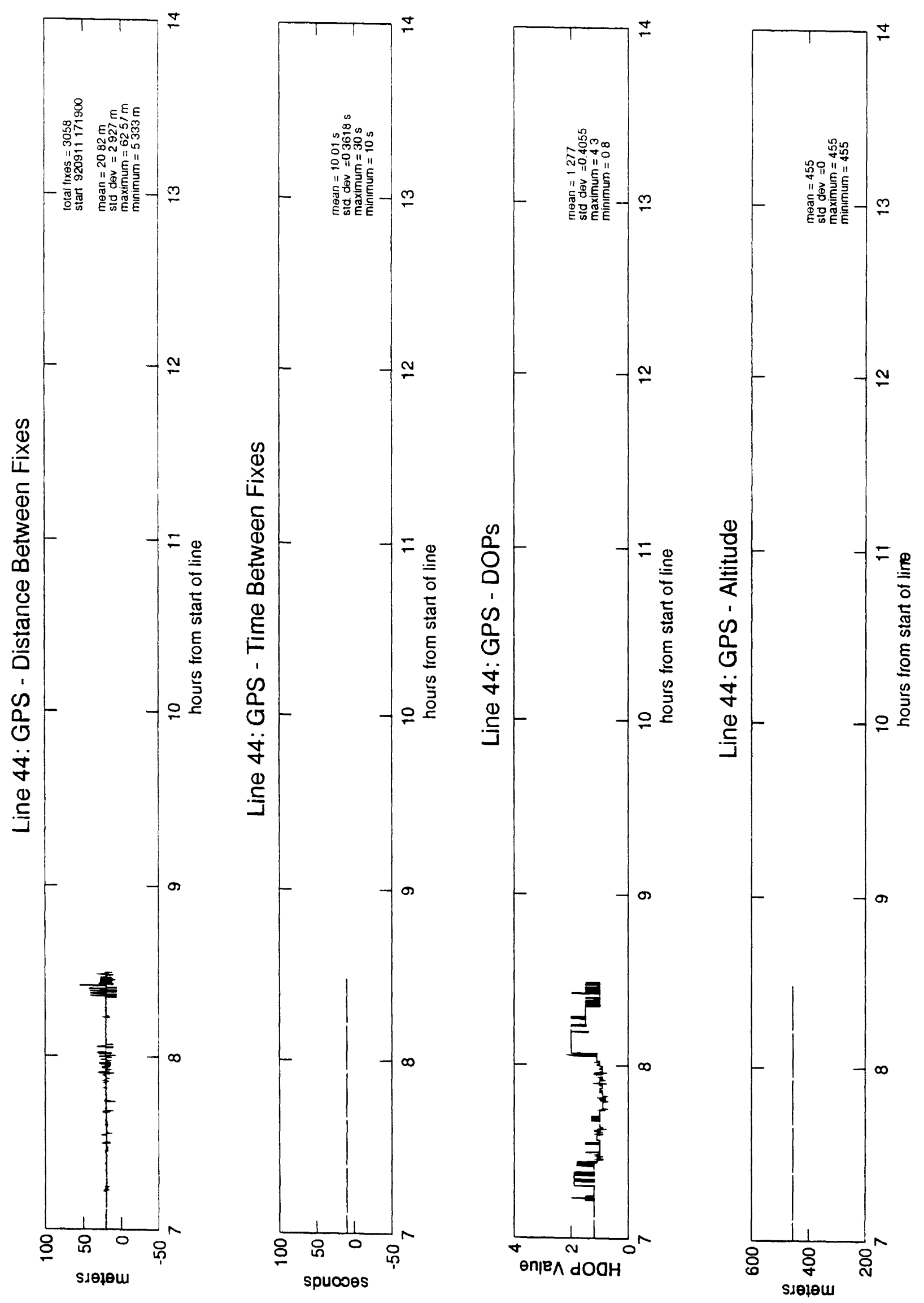

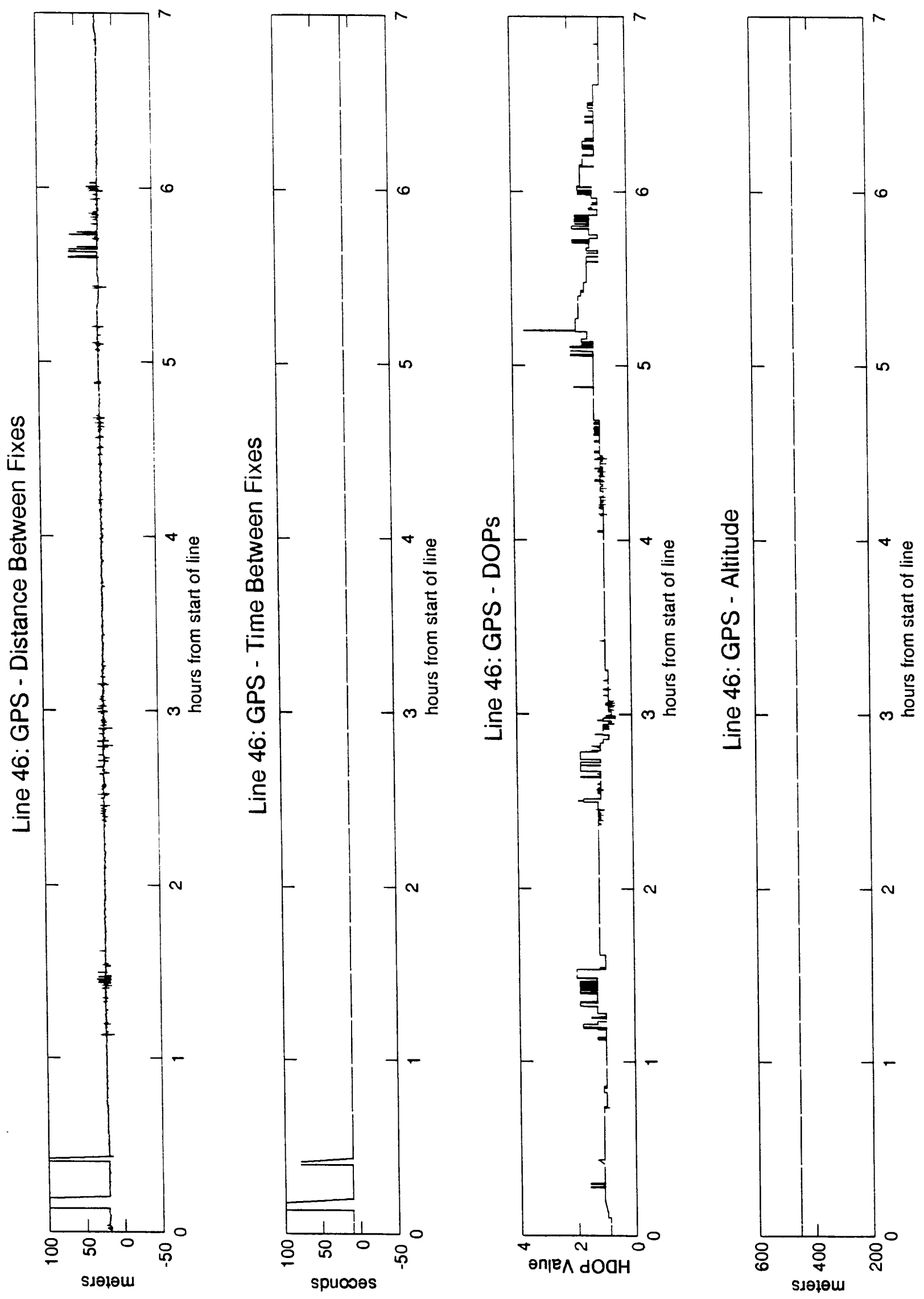

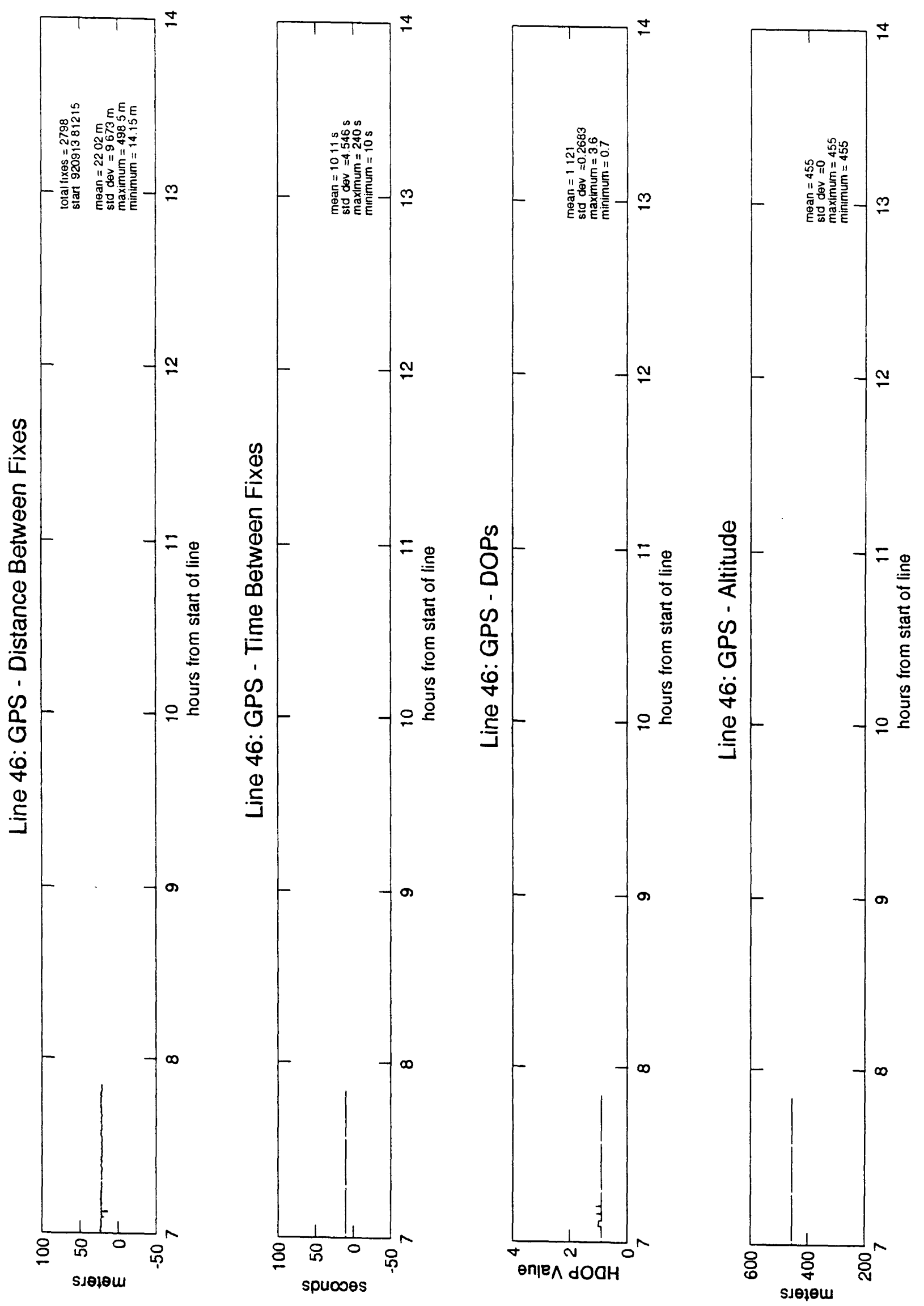

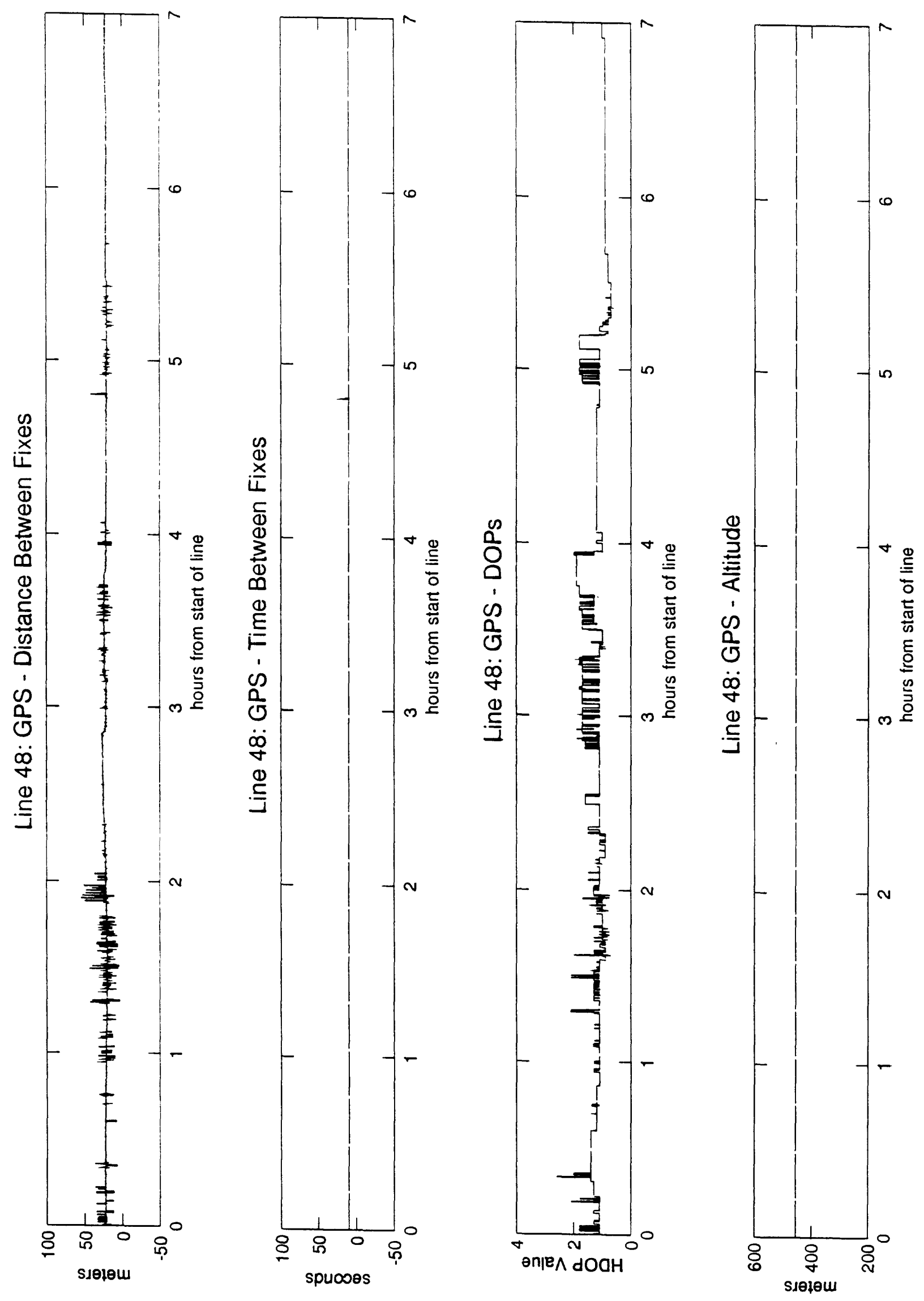

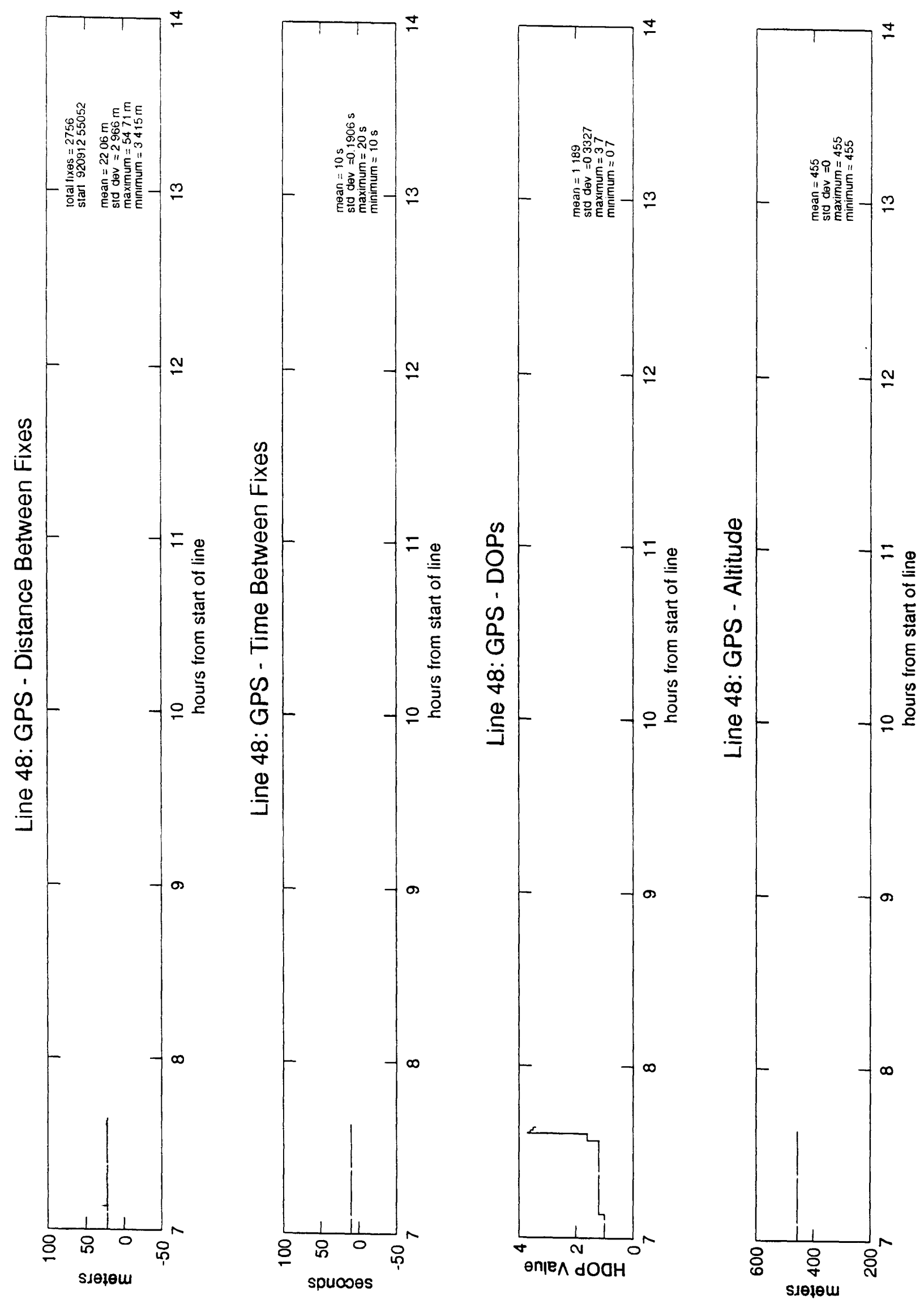

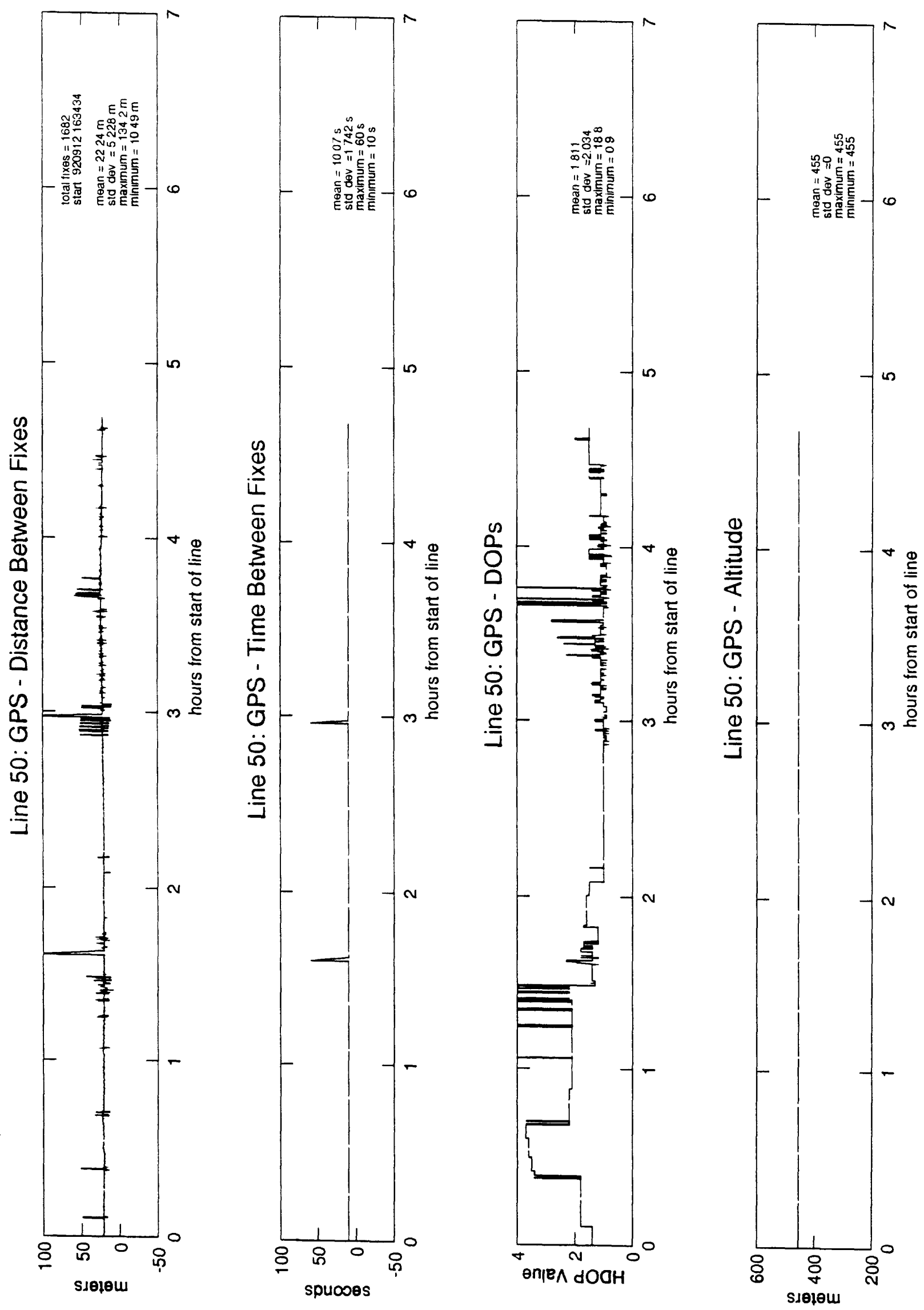

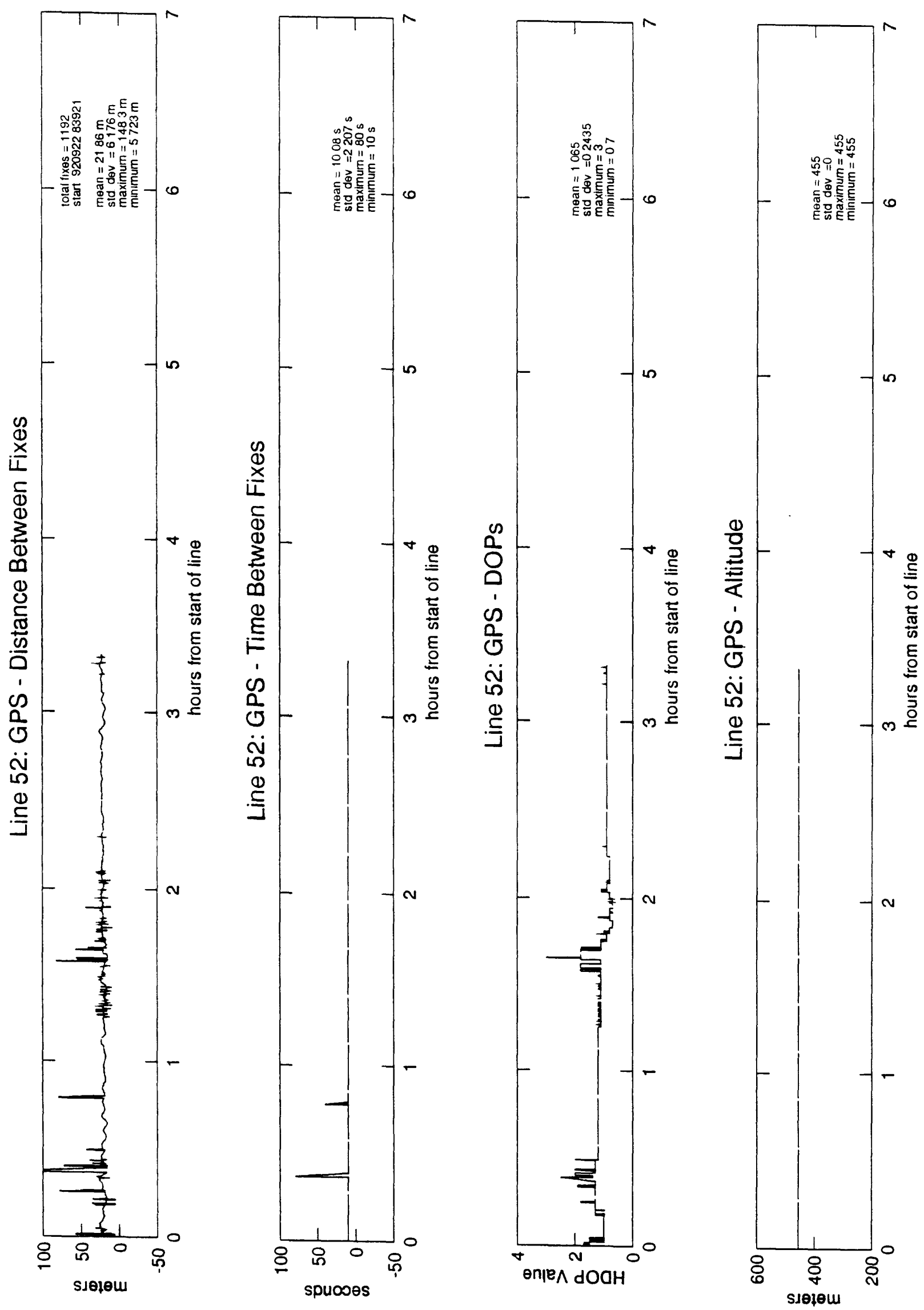

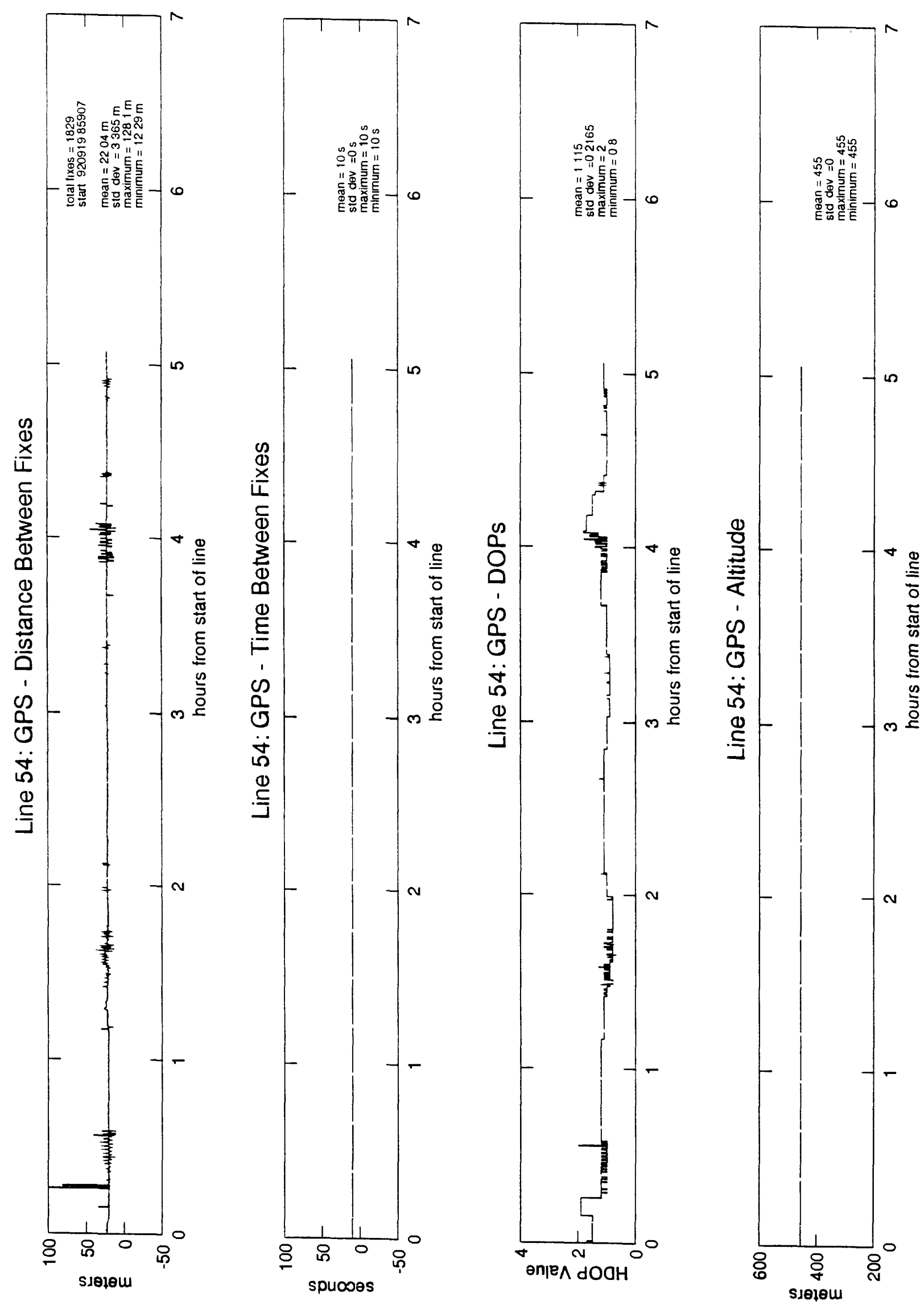

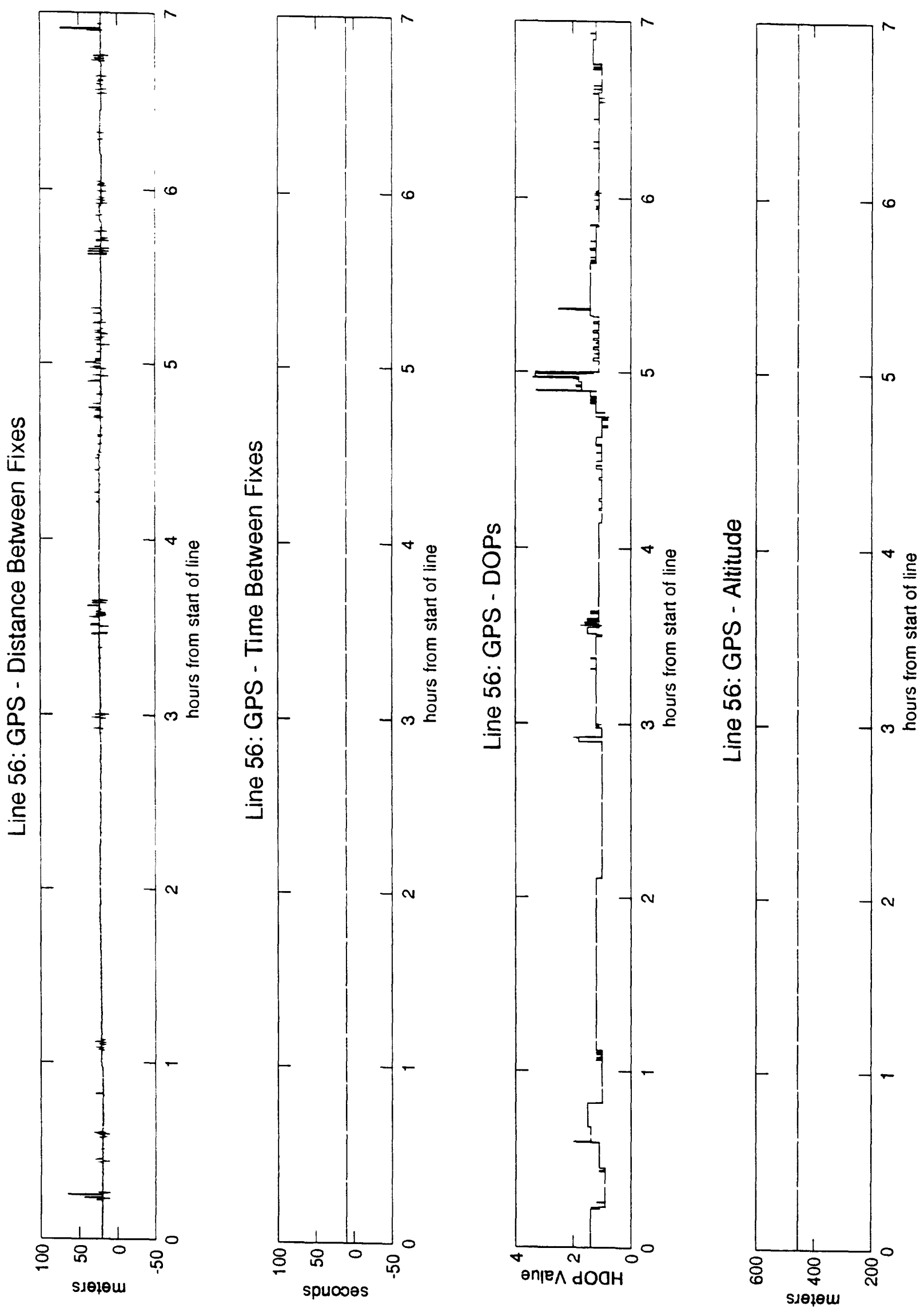

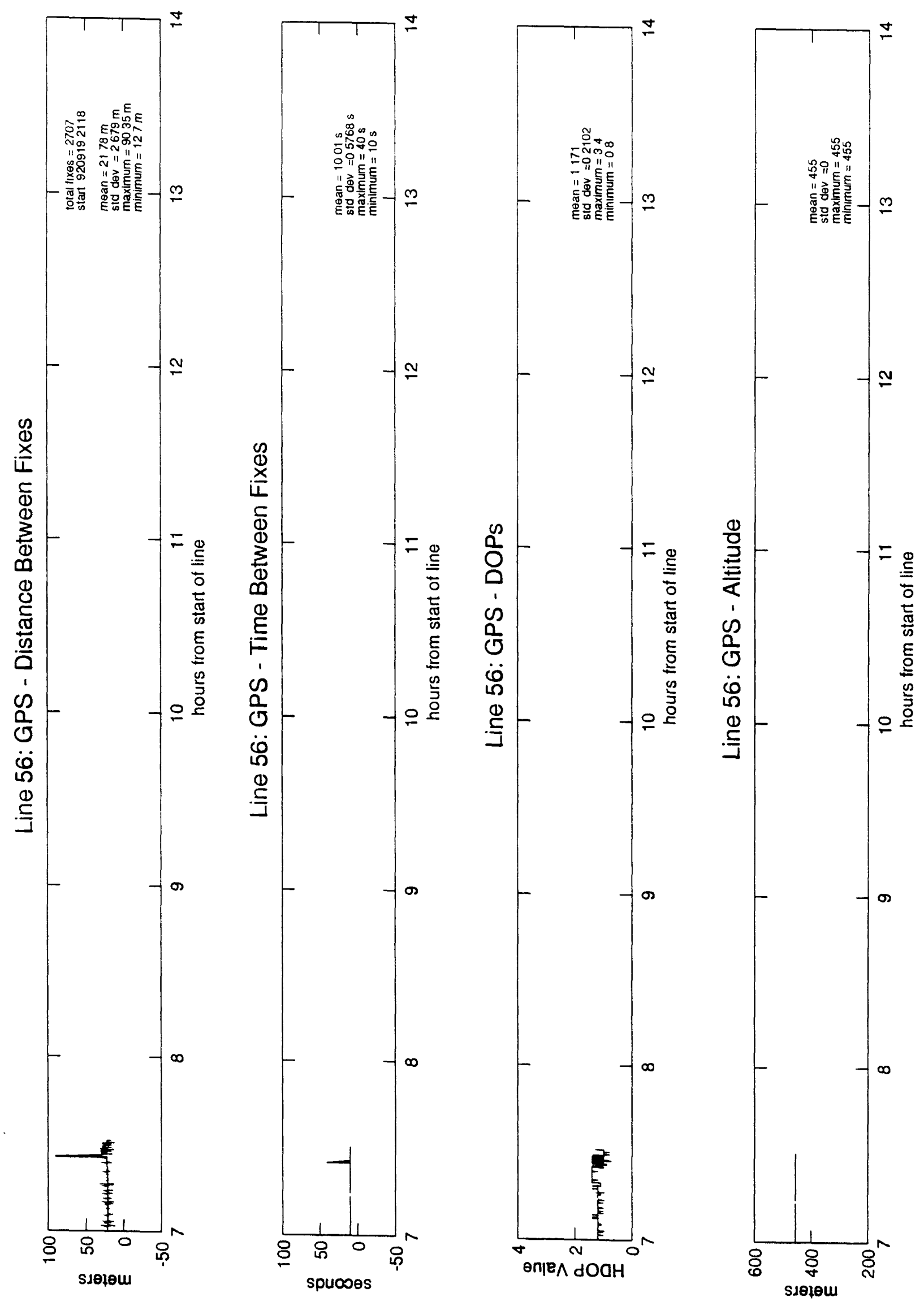

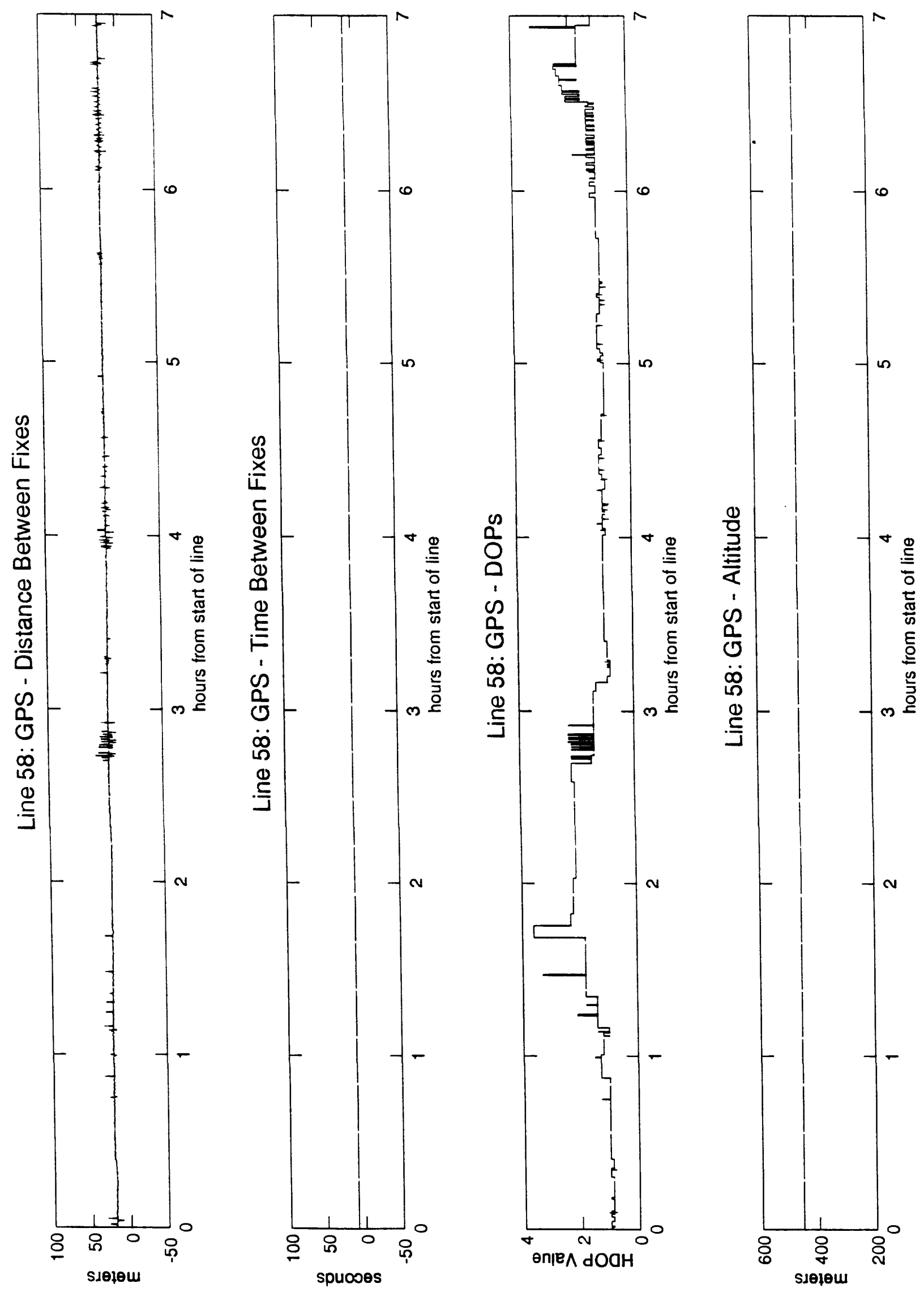

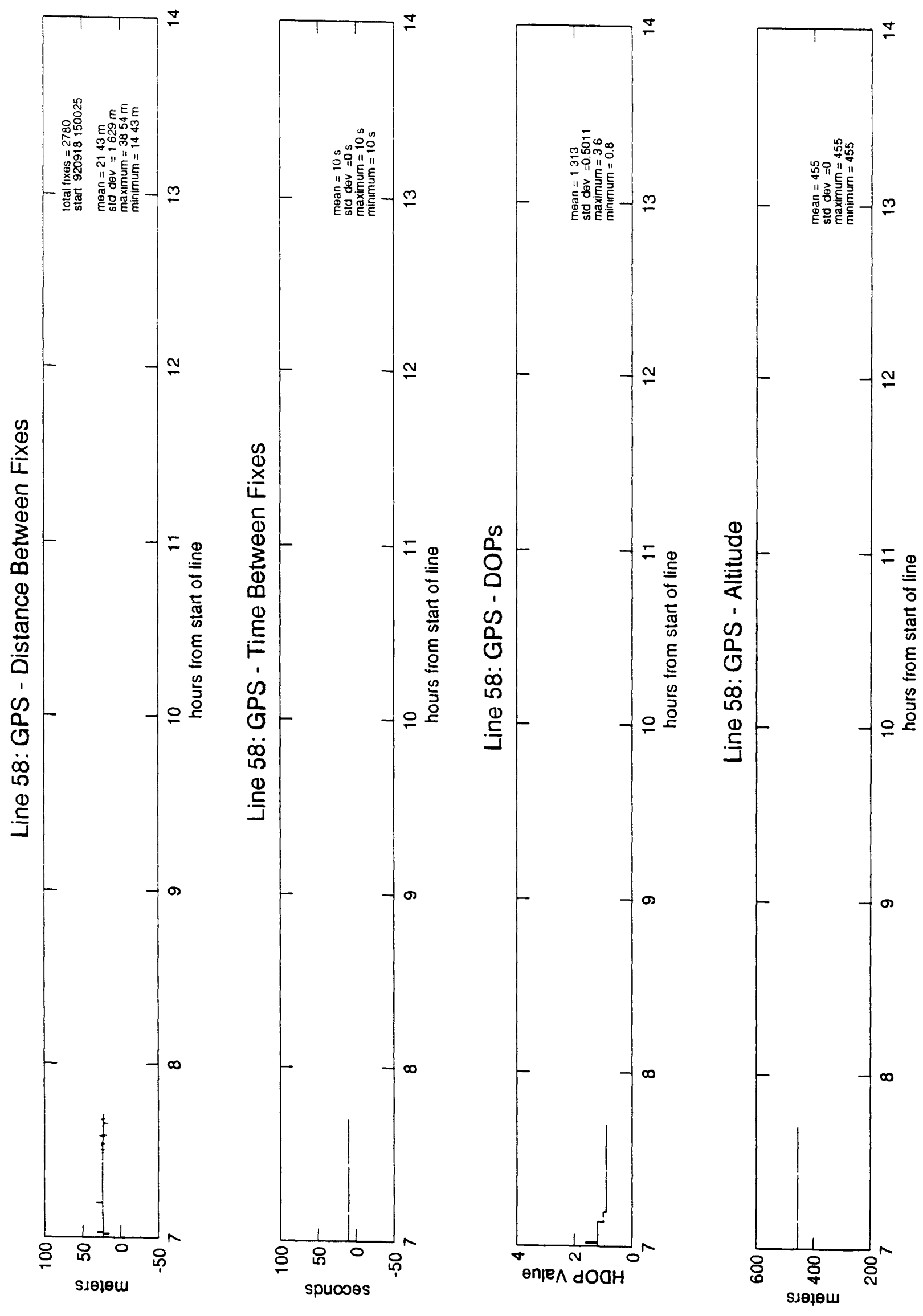

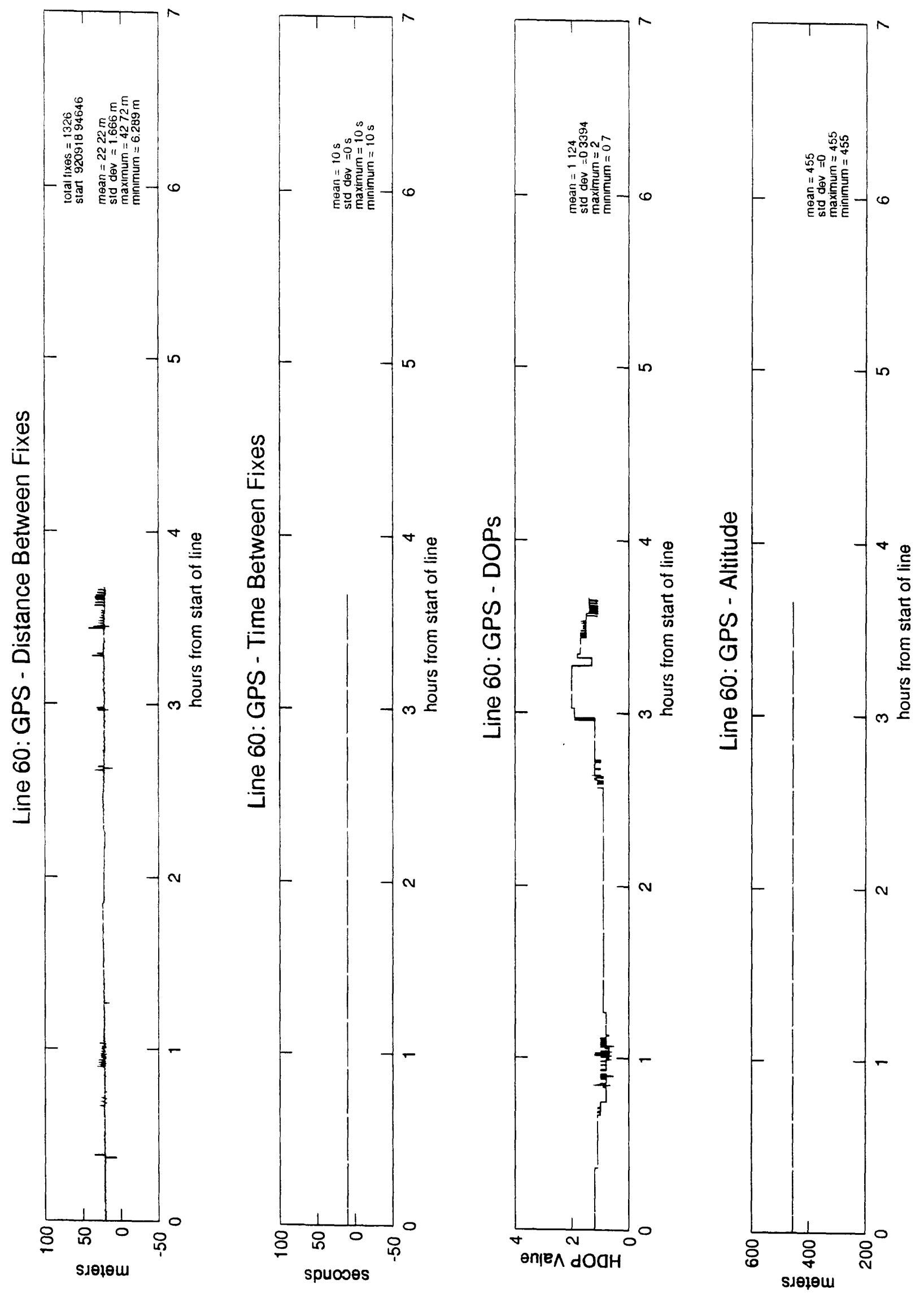

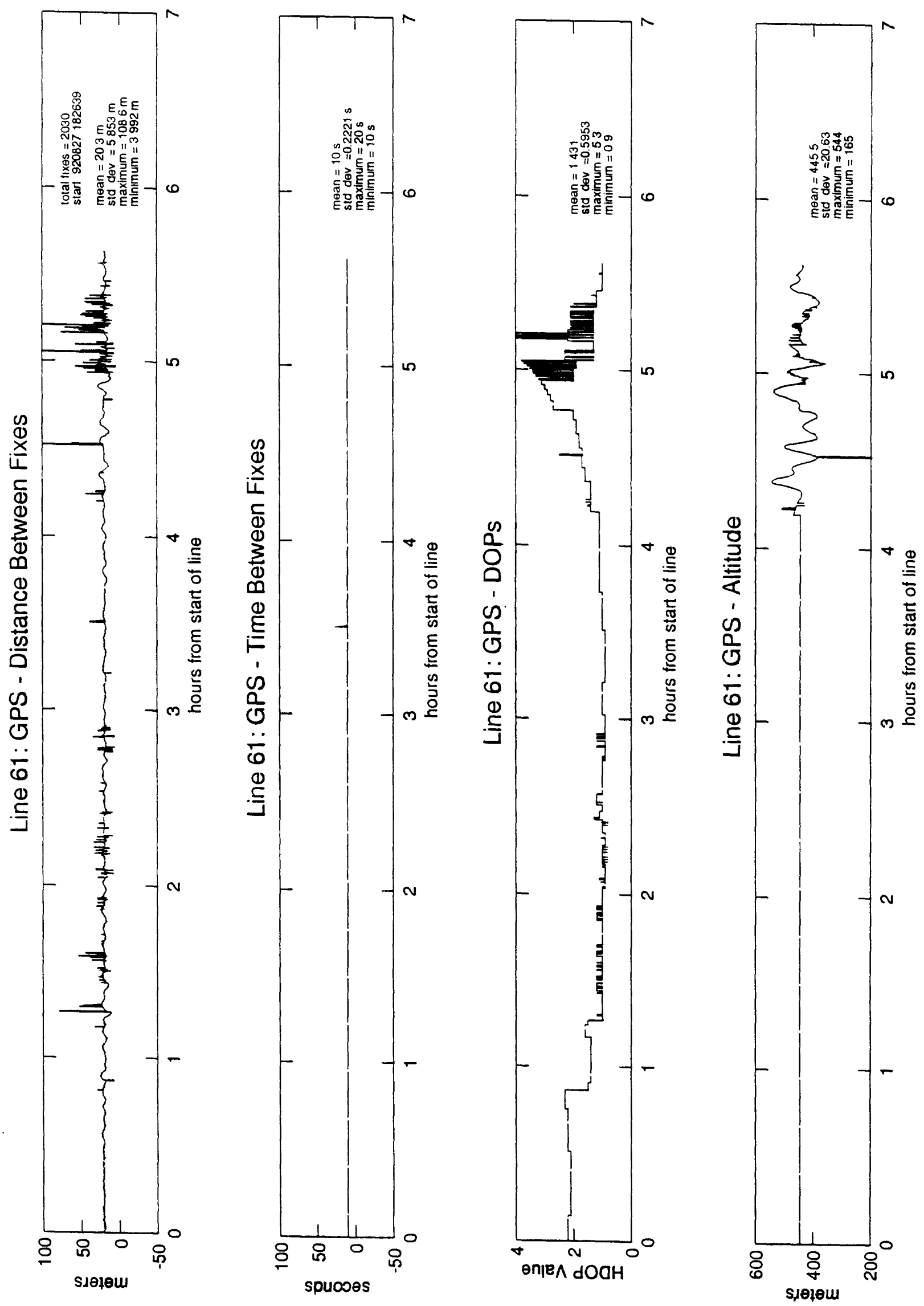

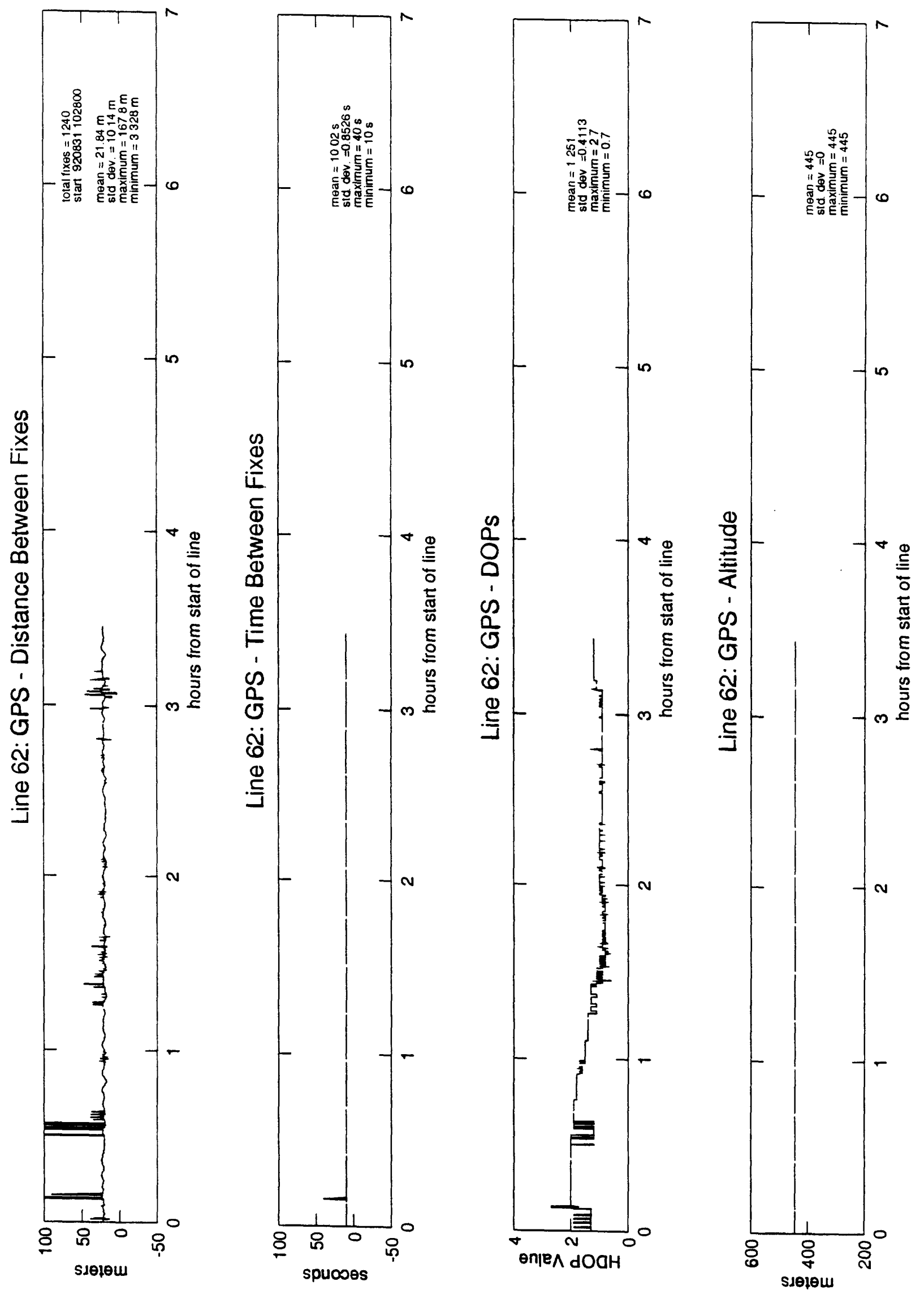

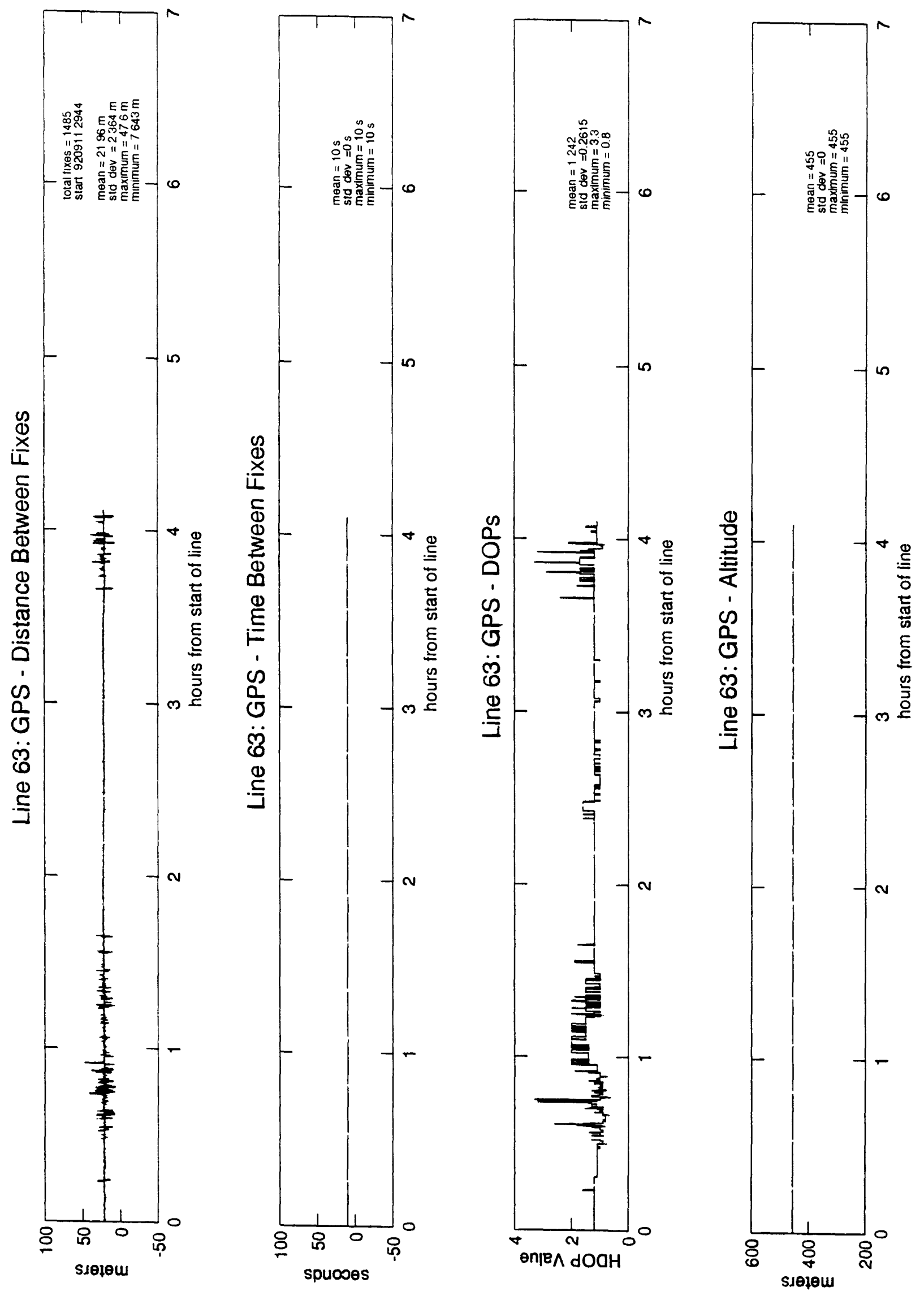

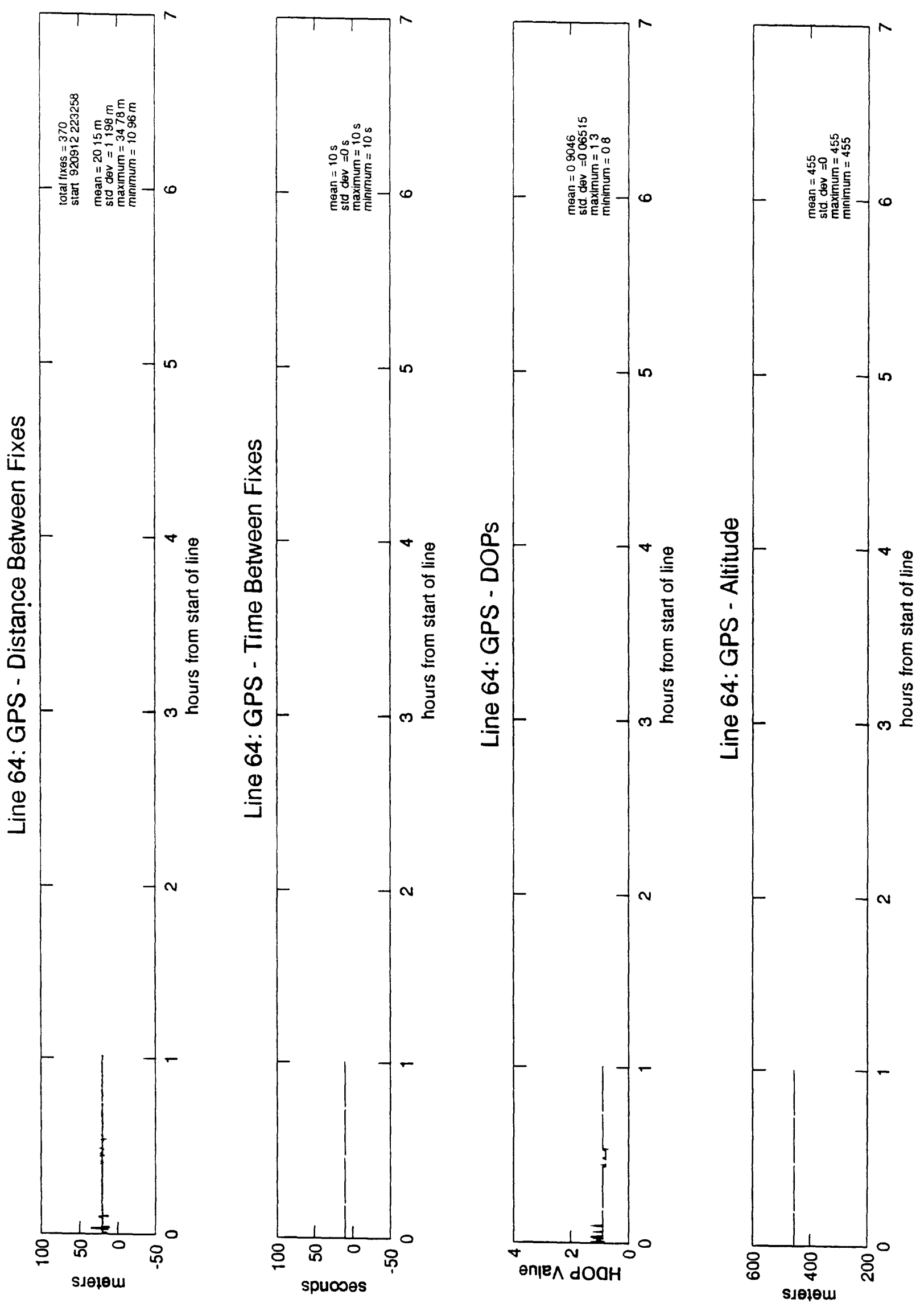

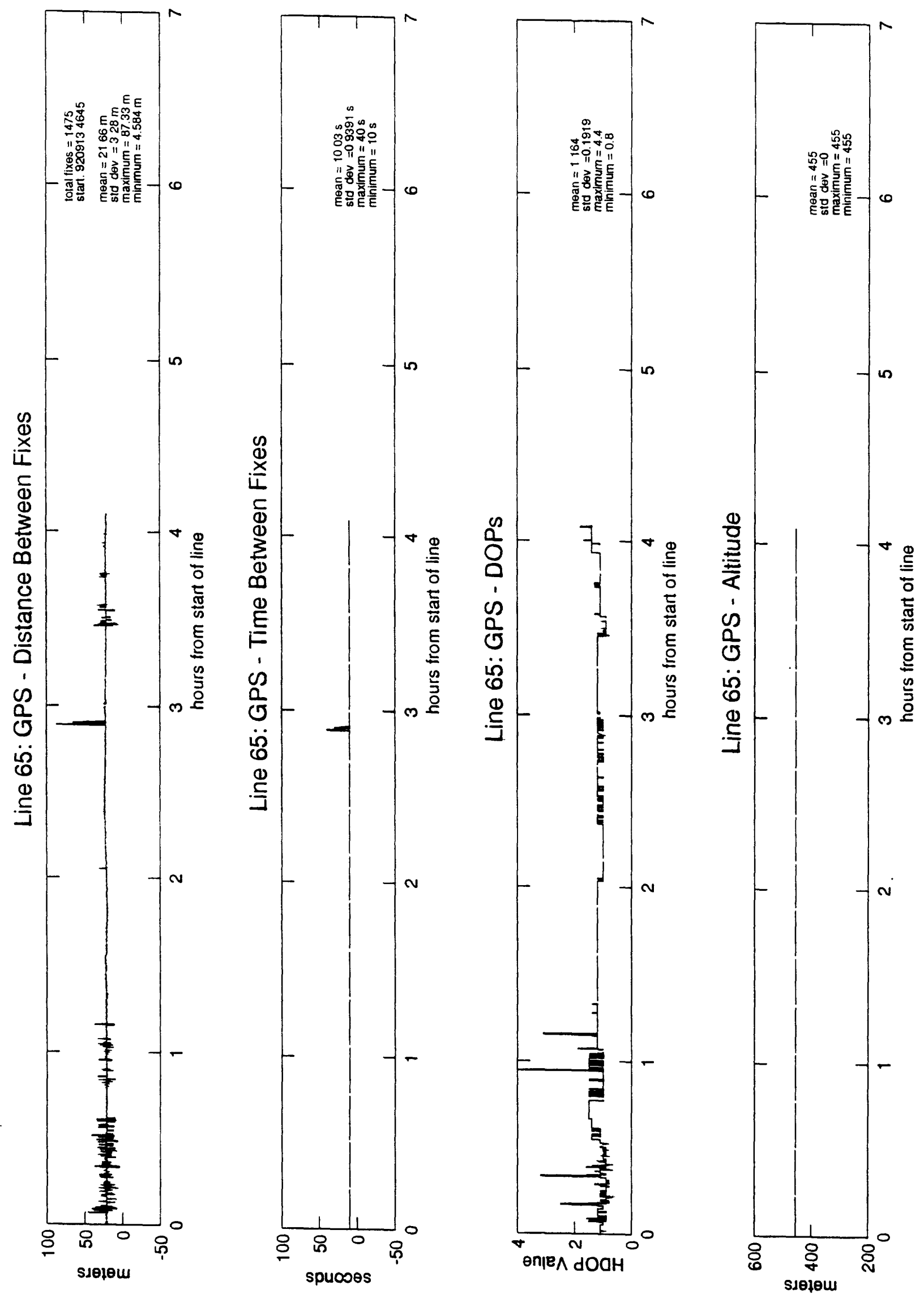

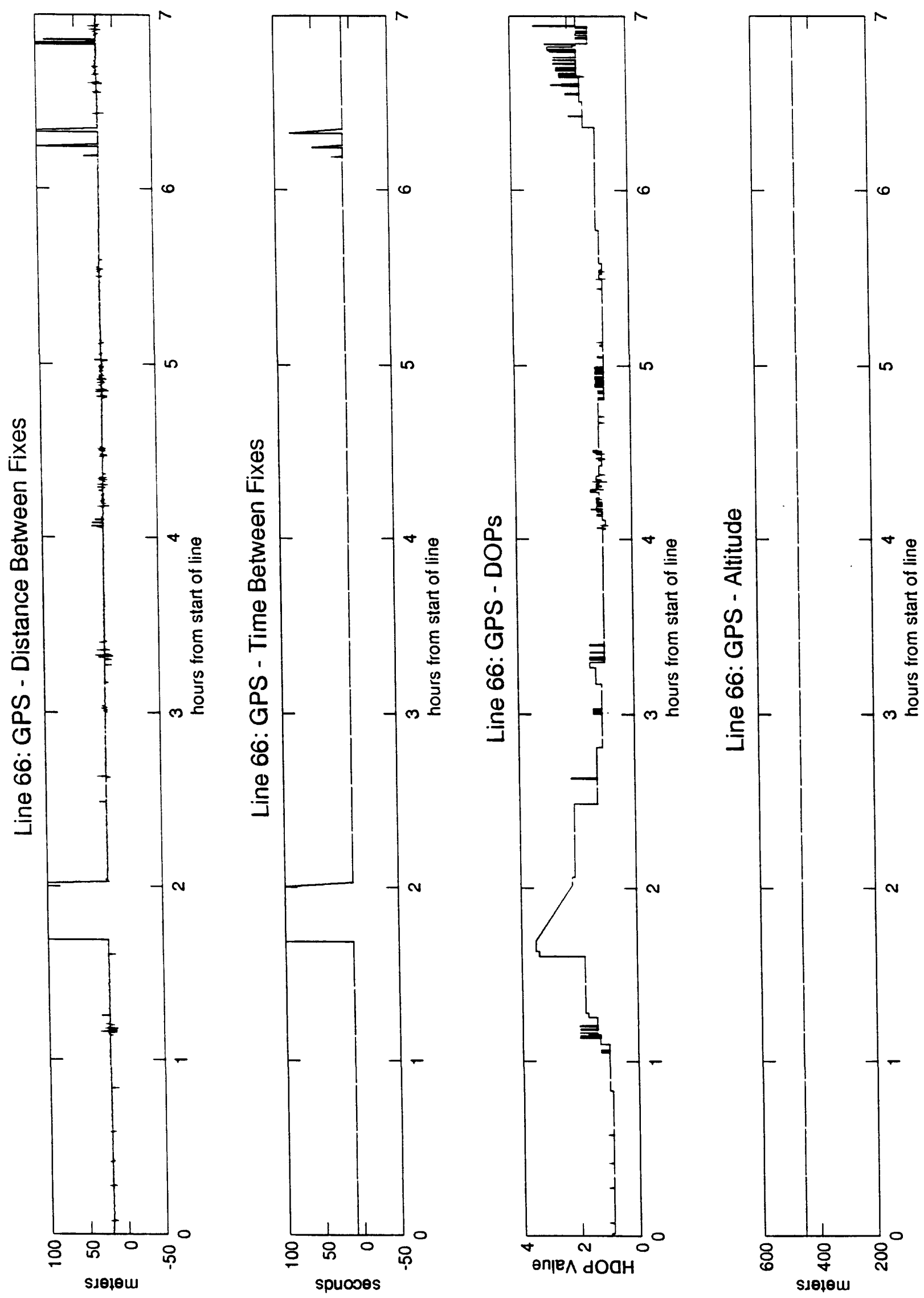

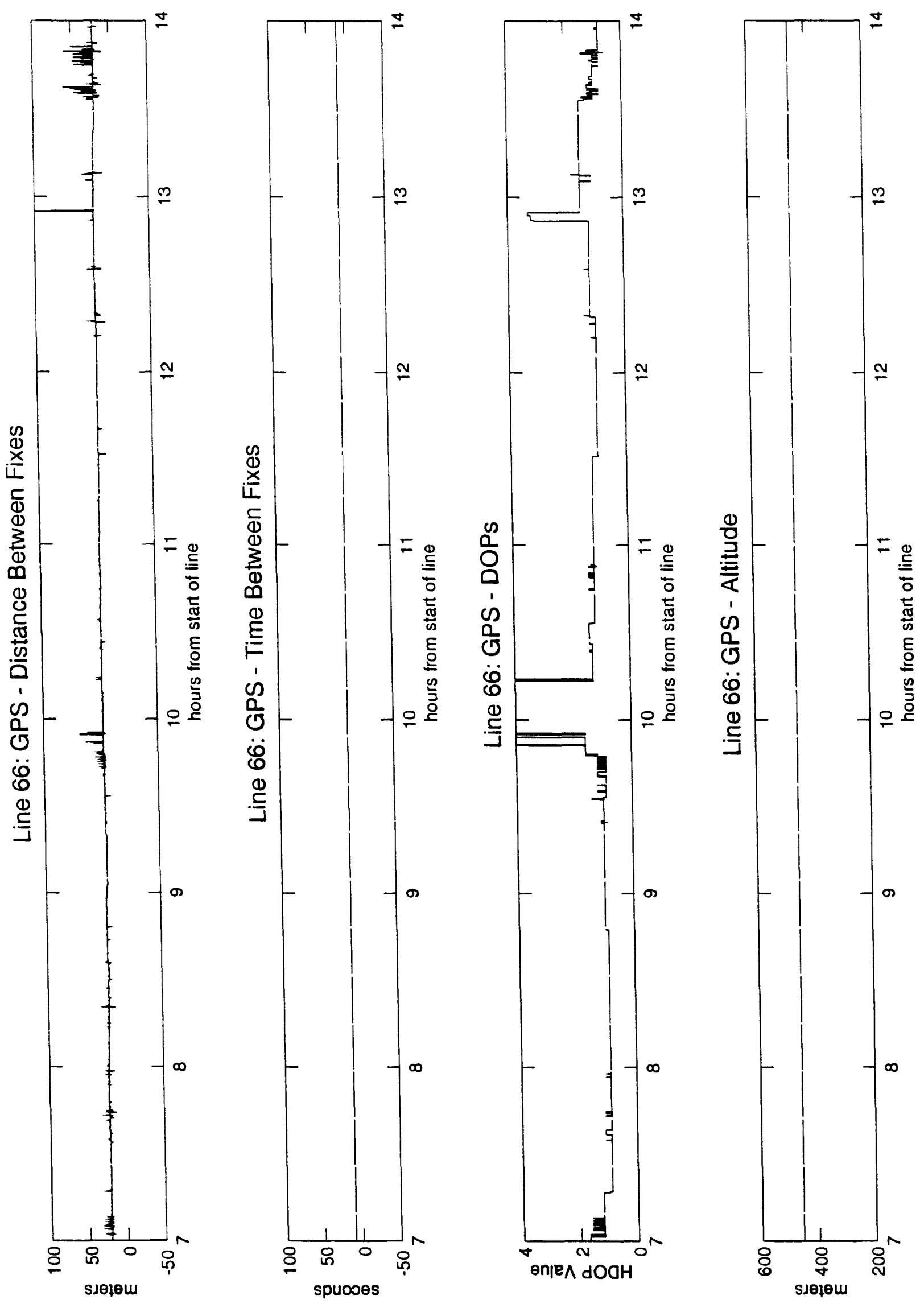

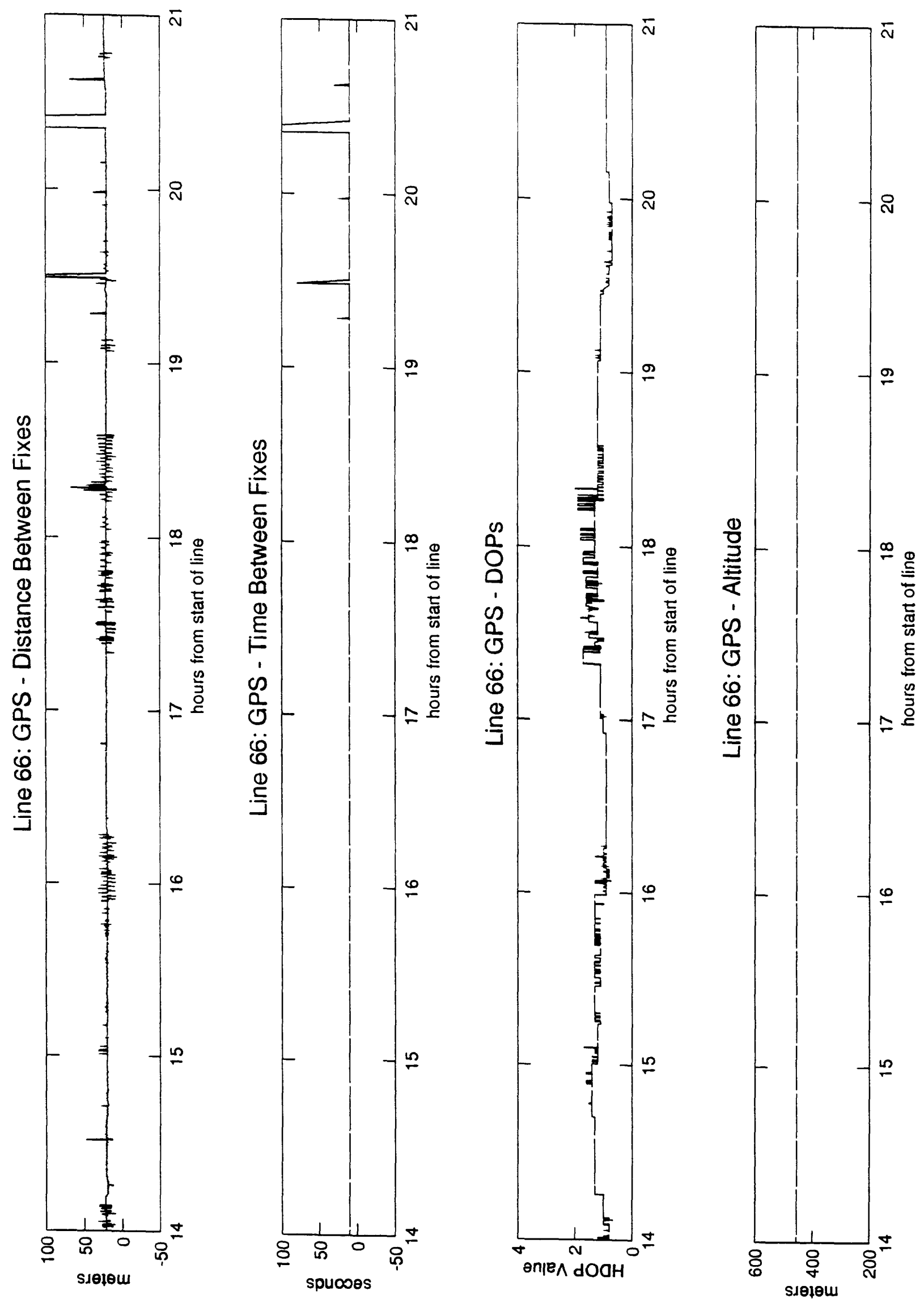

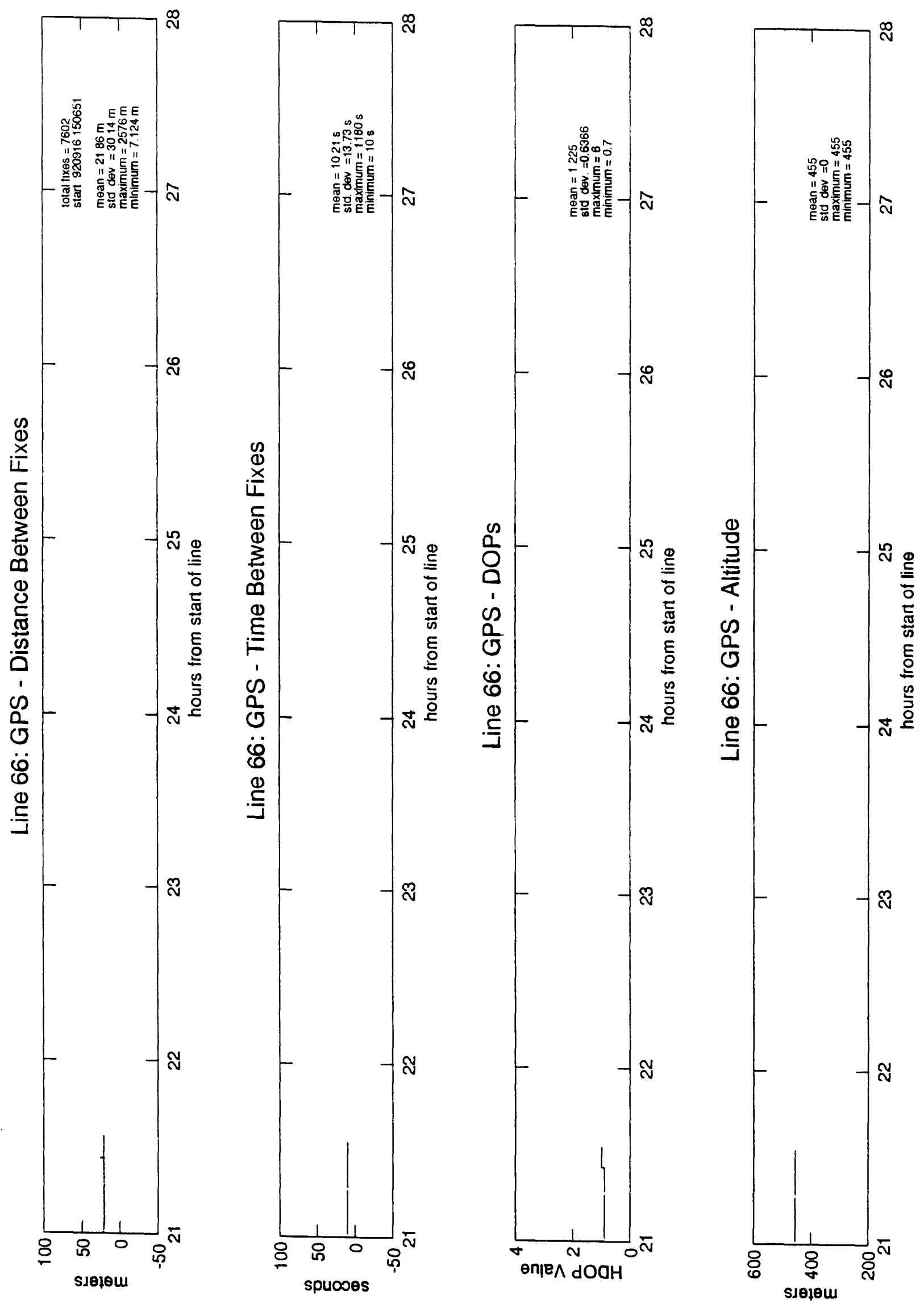


\section{APPENDIX 2}

\section{PLOTS OF SHOT-TIME CALIBRATION}

AND SHOT-DISTANCE INFORMATION 


\section{DESCRIPTION OF SHOT-TIME CALIBRATION AND SHOT-DISTANCE PLOTS}

This appendix gives four plots for each multichannel line from the 1992 Lake Baikal cruise. All plots utilize the same scales, except line 66/17A, which was shot with a different firing rate. Statistics (means and standard deviations) resulting from each step are plotted on each plot, together with a summary of the total shots per line (listed on the histogram plot). Some of the plots are continued on more than one page (lines $7,11,13,15,17$, and 25).

\section{Initial Shot-Time Calibration}

These plots show calculated firing rate versus shot point number. Shot-time calibration was made at 15 -minute intervals (about 35 shots) by logging shot number and a corresponding time. The firing rate was calculated by dividing the number of shots by the time. The locations of the calibration/control points are not labelled but can be extracted from most of the plots at the positions where the calculated firing rate changes, causing the boxy appearance of the plots. Initial shot time plots do not account for edits due to logging errors and missed shots. Logging of time to whole minutes is noted on the appropriate plots (Lines $1,2,3,4,6,7,8,10,16,20$, $22,61 / 10 \mathrm{~A}, 62 / 3 \mathrm{~A})$.

\section{Final Shot-Time Calibration}

These plots are identical to initial shot-time calibration plots, except that edits for incorrect logging and missed shots are included in the firing rate calculations. The positions of whole-minute logging are noted the same as for the initial shot-time calibration plots. Ideally, the plots of firing rate should be flat, because the airgun array was fired on even increments of time. Examples of reasonably flat final curves are lines 9, 11, 12, 13 (SP $1500-2500$ ), 19, 25, $26,32,34,52,54,56,58$, and 63 . Examples of lines where editing provided major improvement in the calculated firing rate are lines $2,11,13,19,25,34,52,54,56,58$, and $66 / 17 \mathrm{~A}$. The boxiness of many of the "final" plots indicates poor final control on the firing rate (e.g., lines $4,15,17,21,42,44,46,48$, and 50).

\section{Distance Between Shots}

These plots show the calculated distance between shots estimated after merging the raw navigation with the final shot-time calibration files to produce an interpolated position and time for each shot point. Missing shots (i.e., pops) are included in this plot so that spikes that would otherwise be caused by missing shots do not show up. Pops are also included in the statistics (means and standard deviations).

\section{Histogram of Distance Between Shots}

This plot shows the distribution of the scatter associated with the distance between shots. The mean distance is plotted as a ${ }^{*}$ on this plot. For lines that plot on multiple pages, the histogram plot is plotted only once, on the first page. 


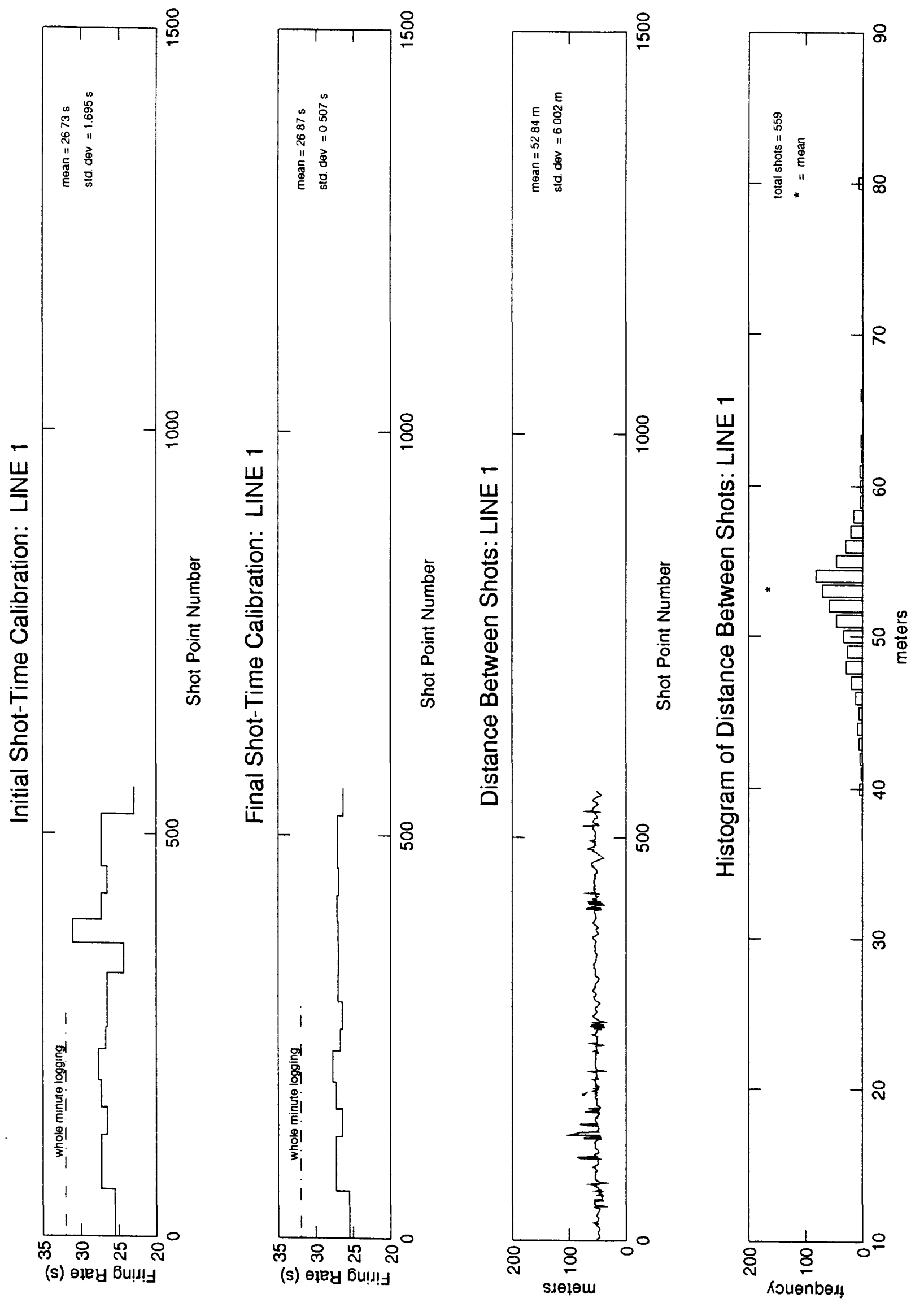




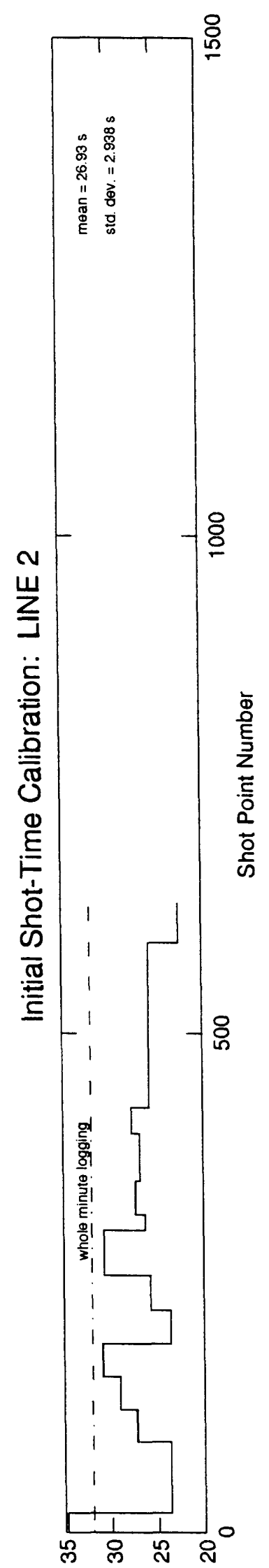

(s) ө1ey 6u!!!y
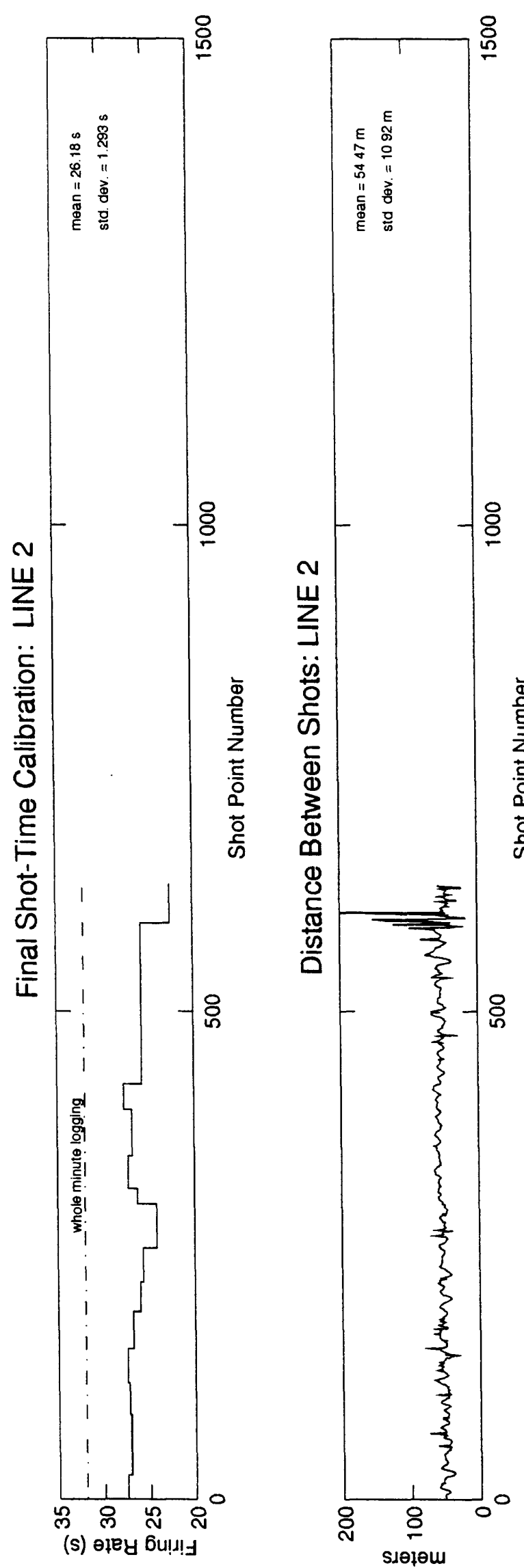

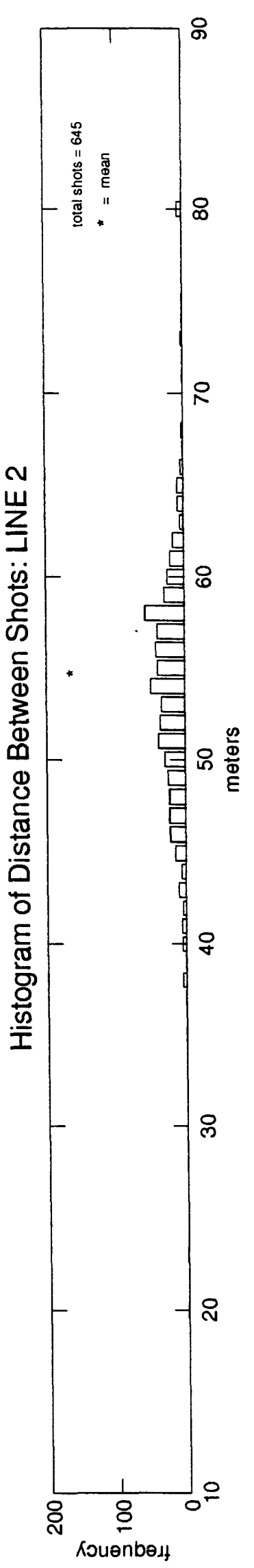



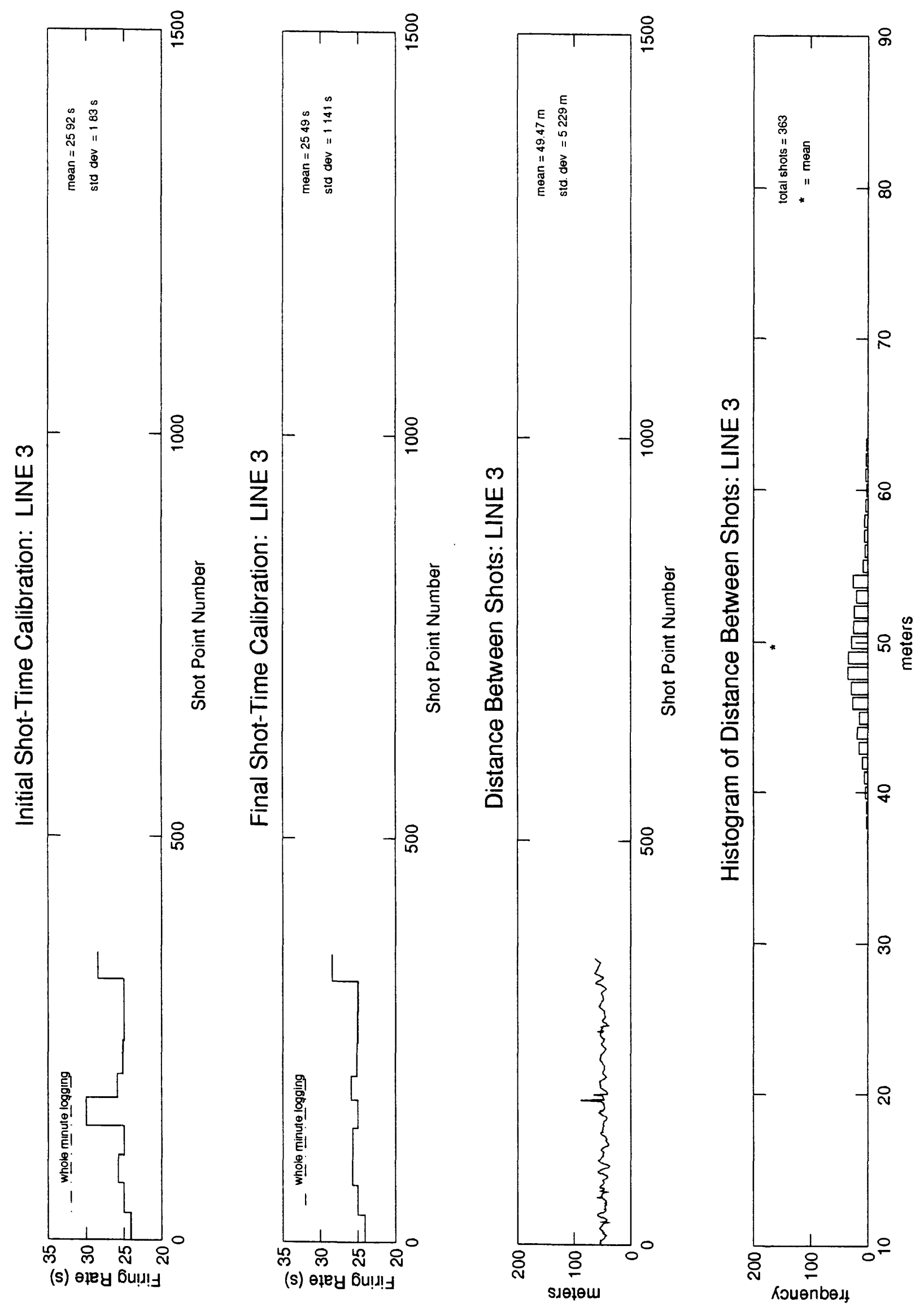


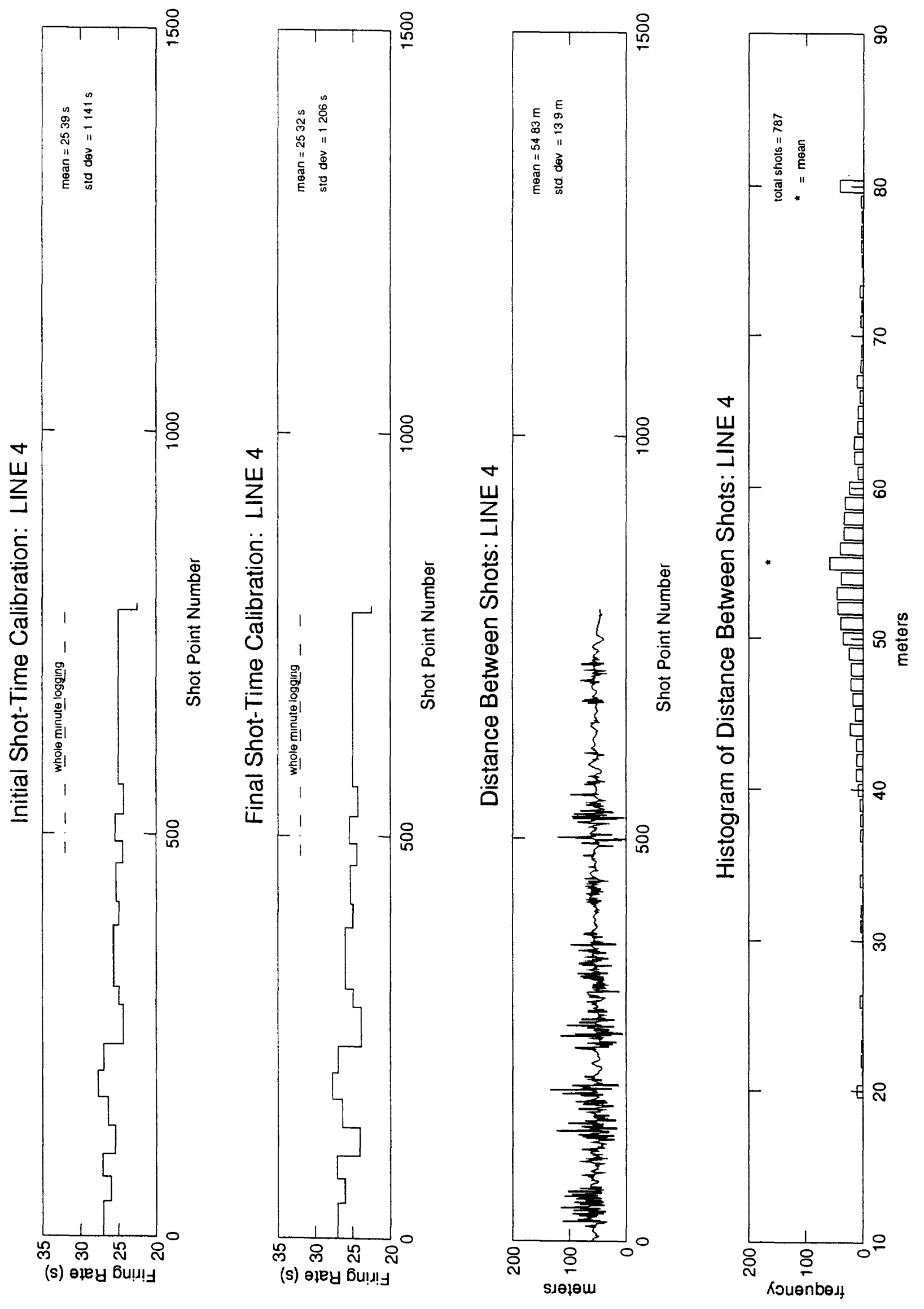




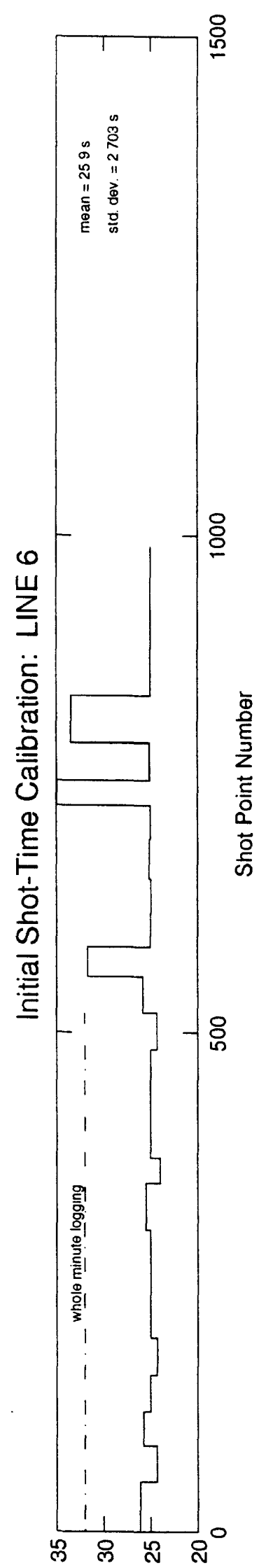

(s) өley bu!n!
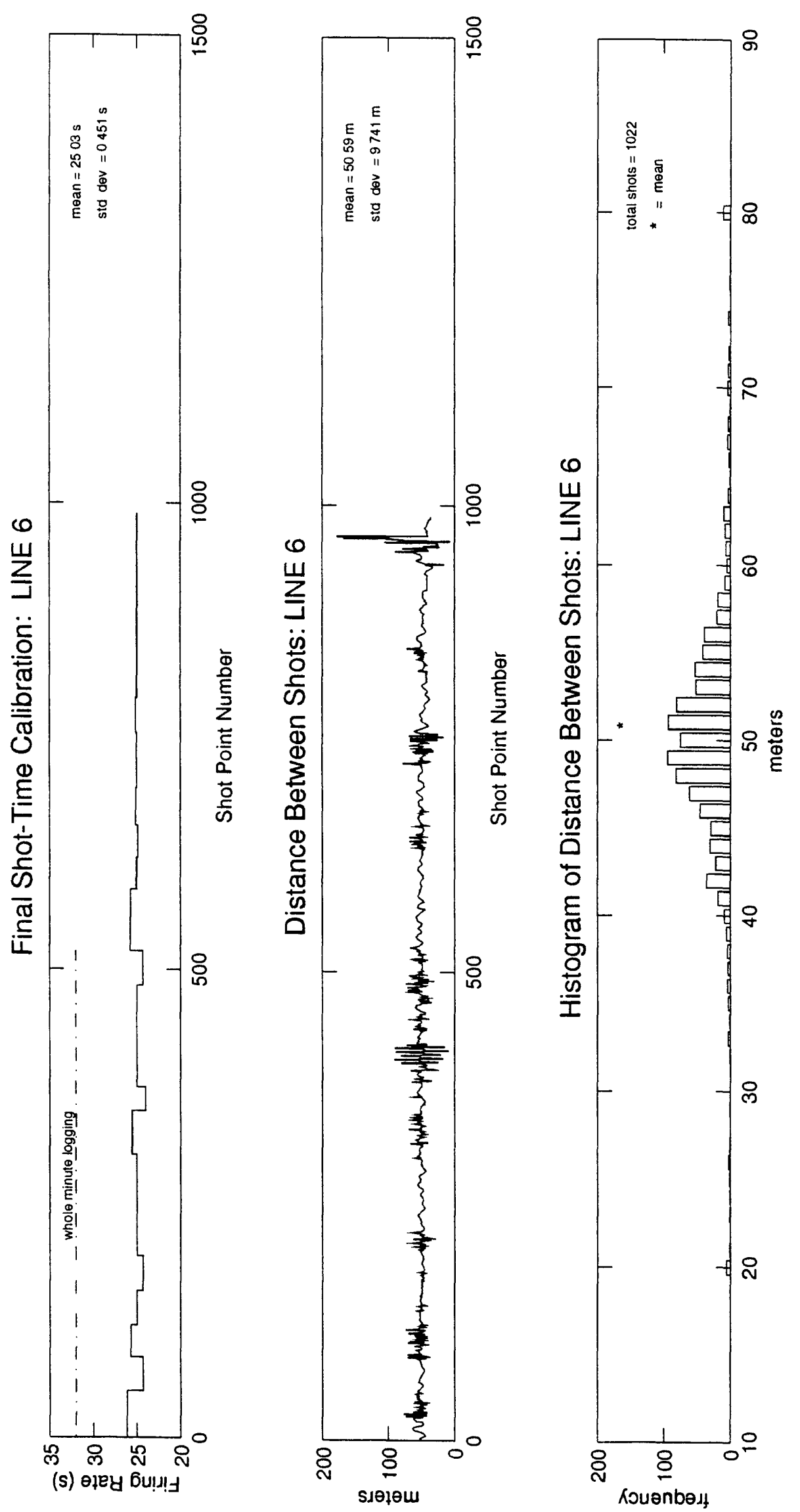

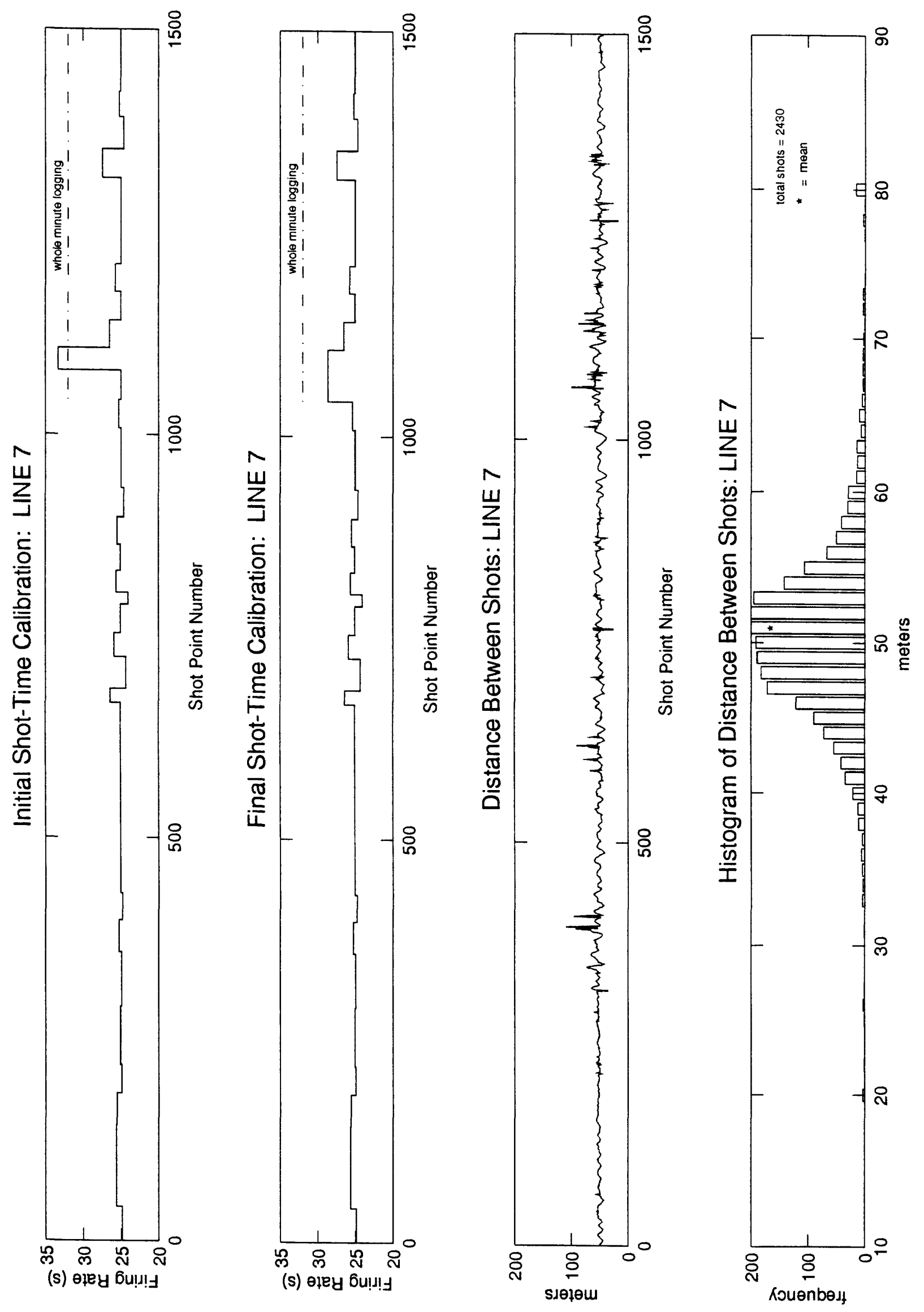


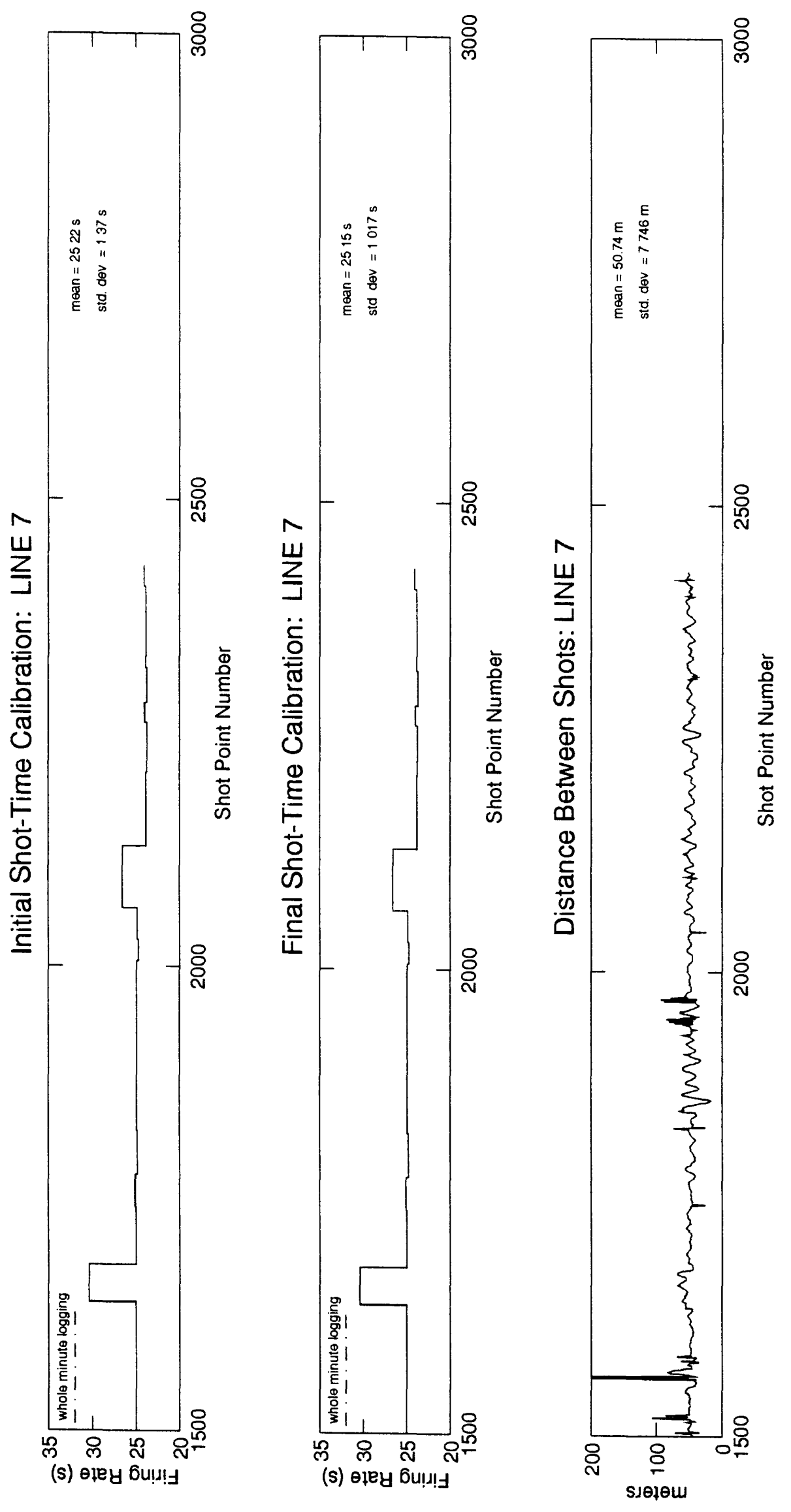



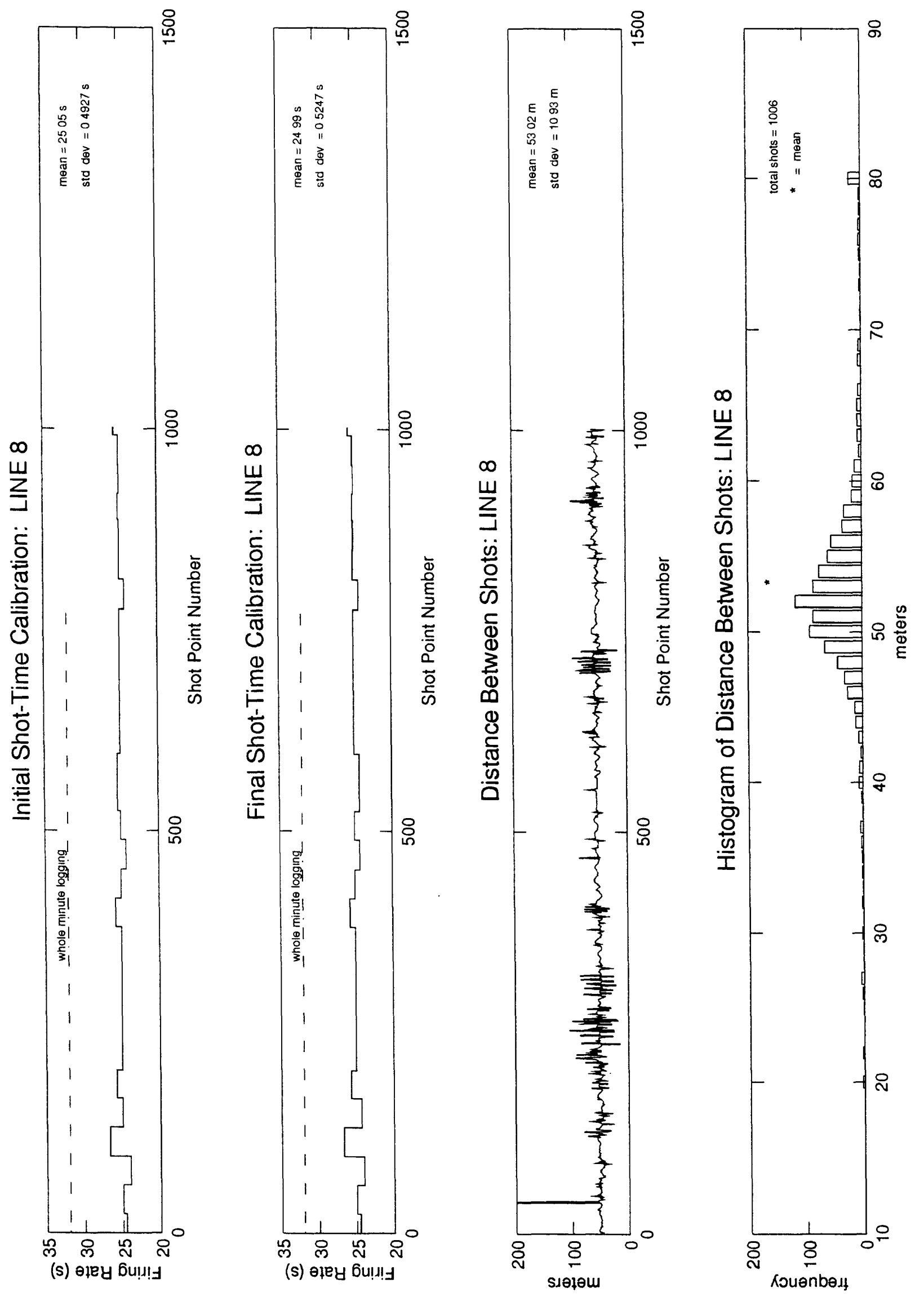

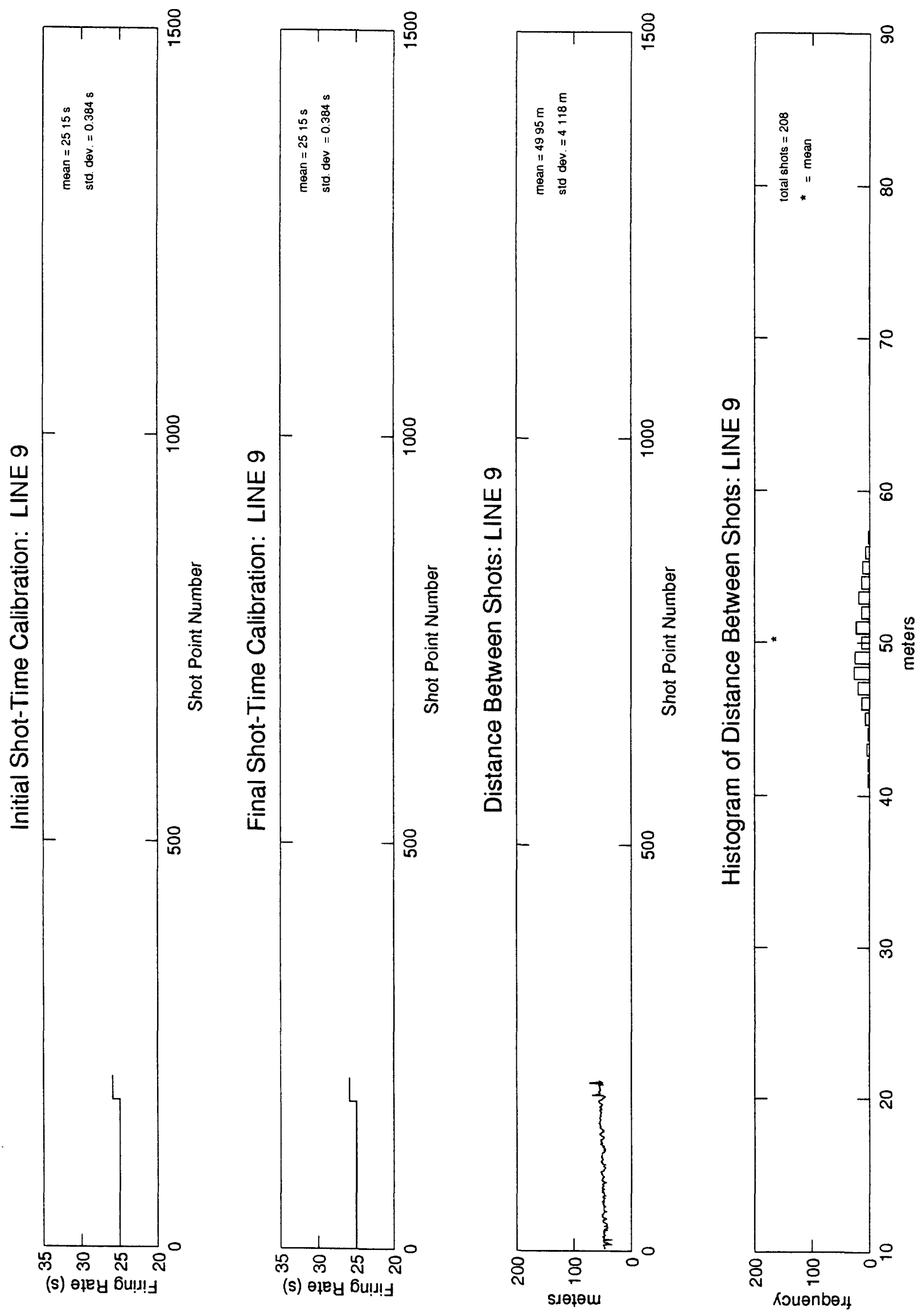

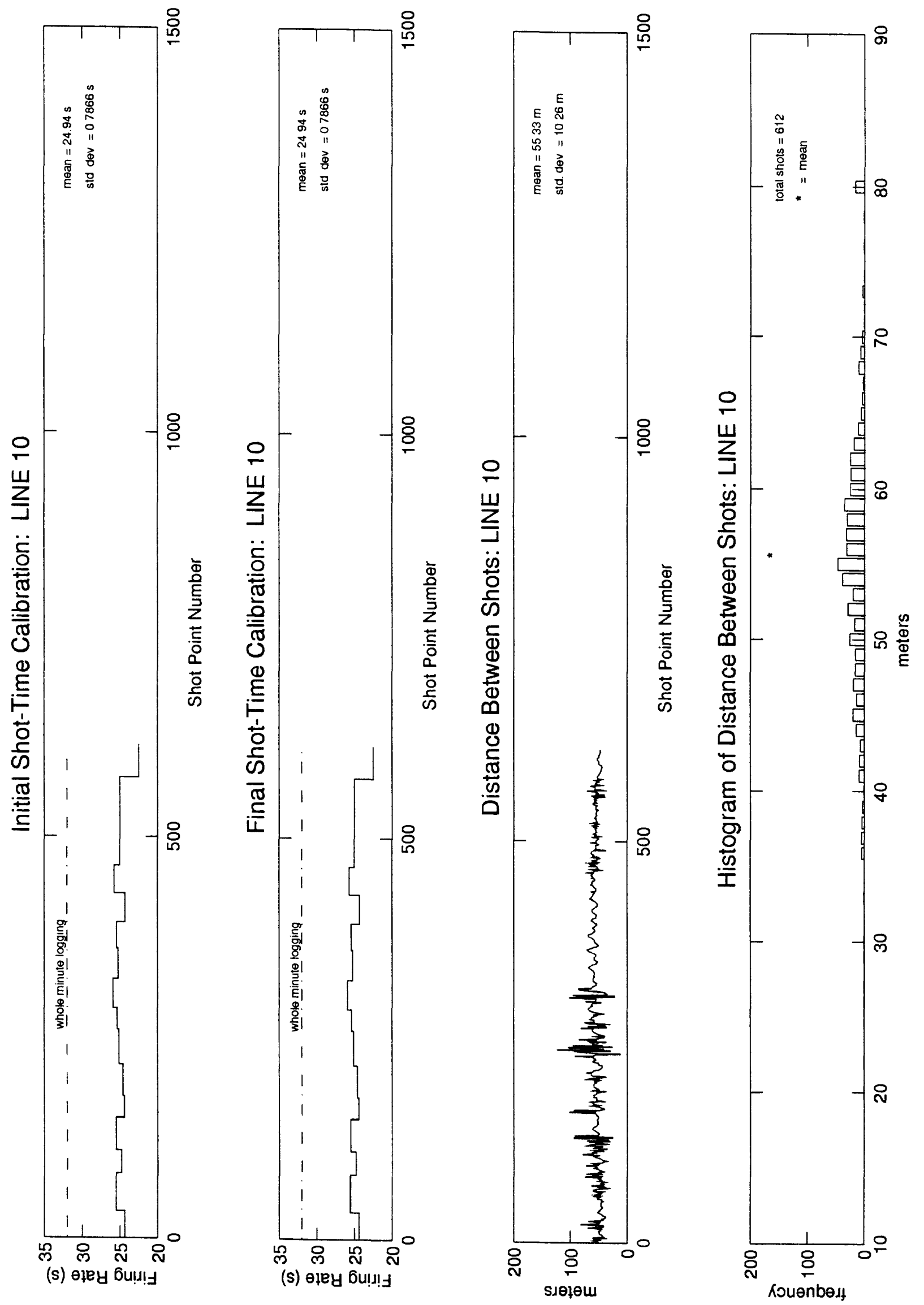

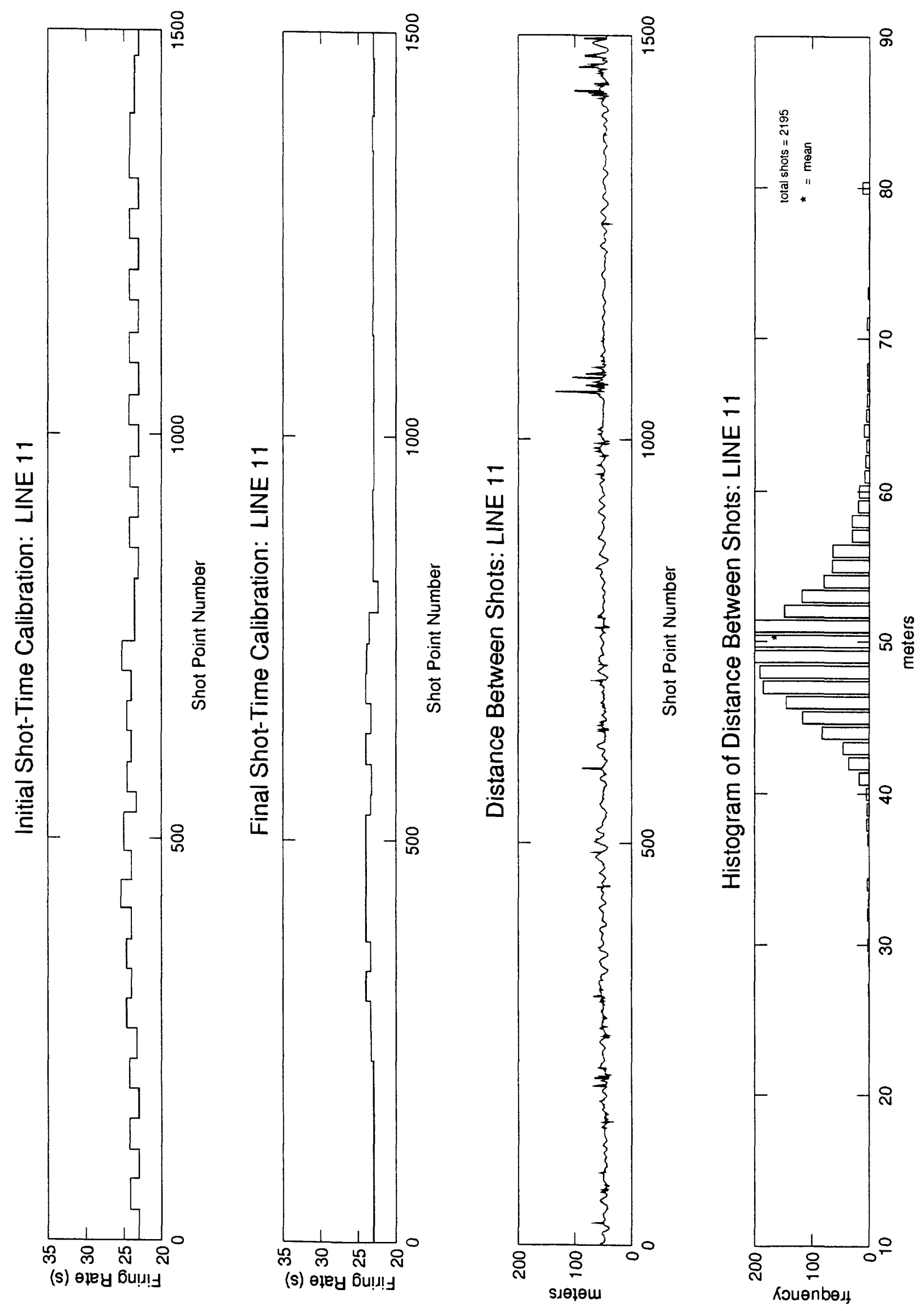

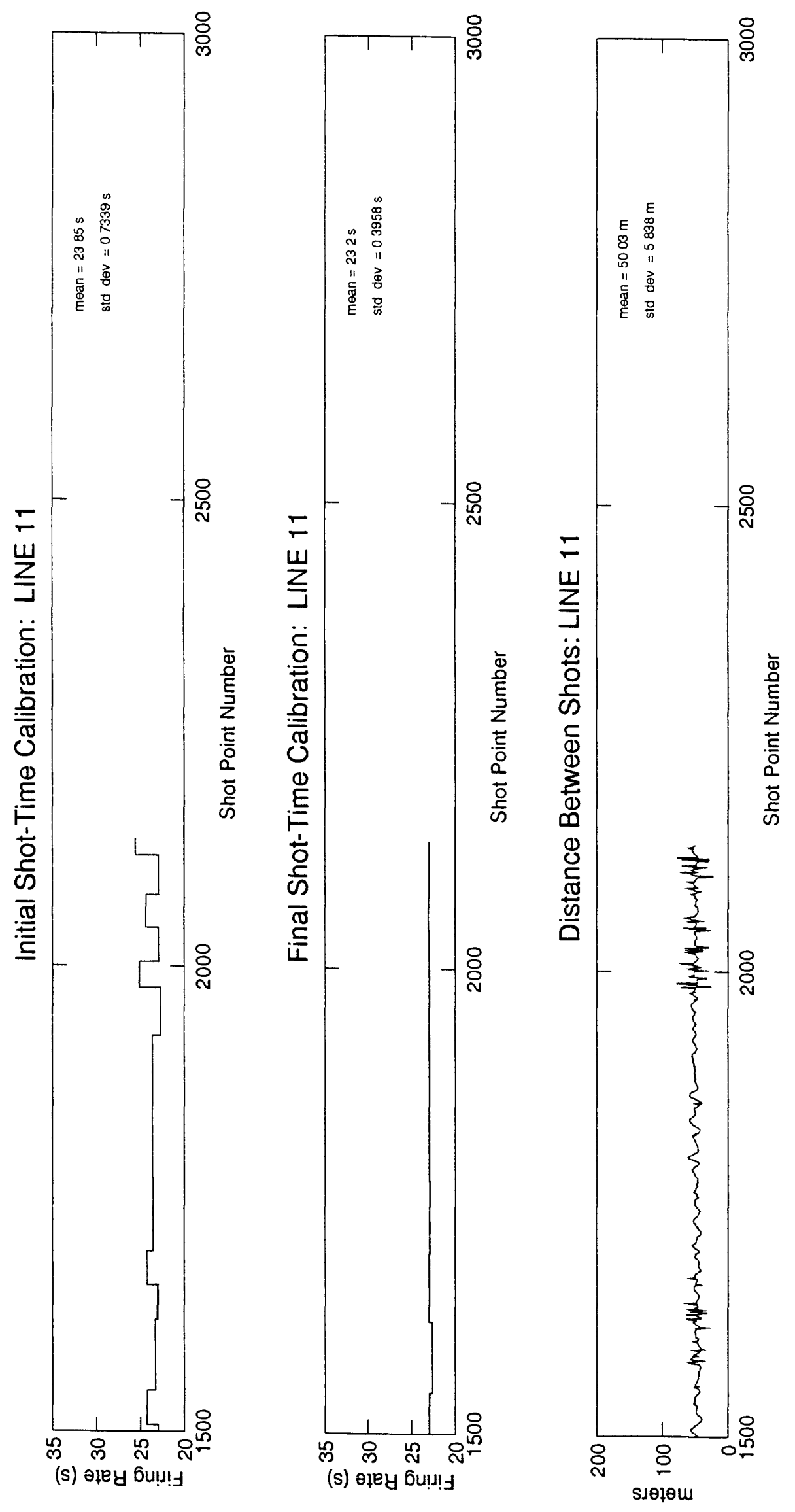


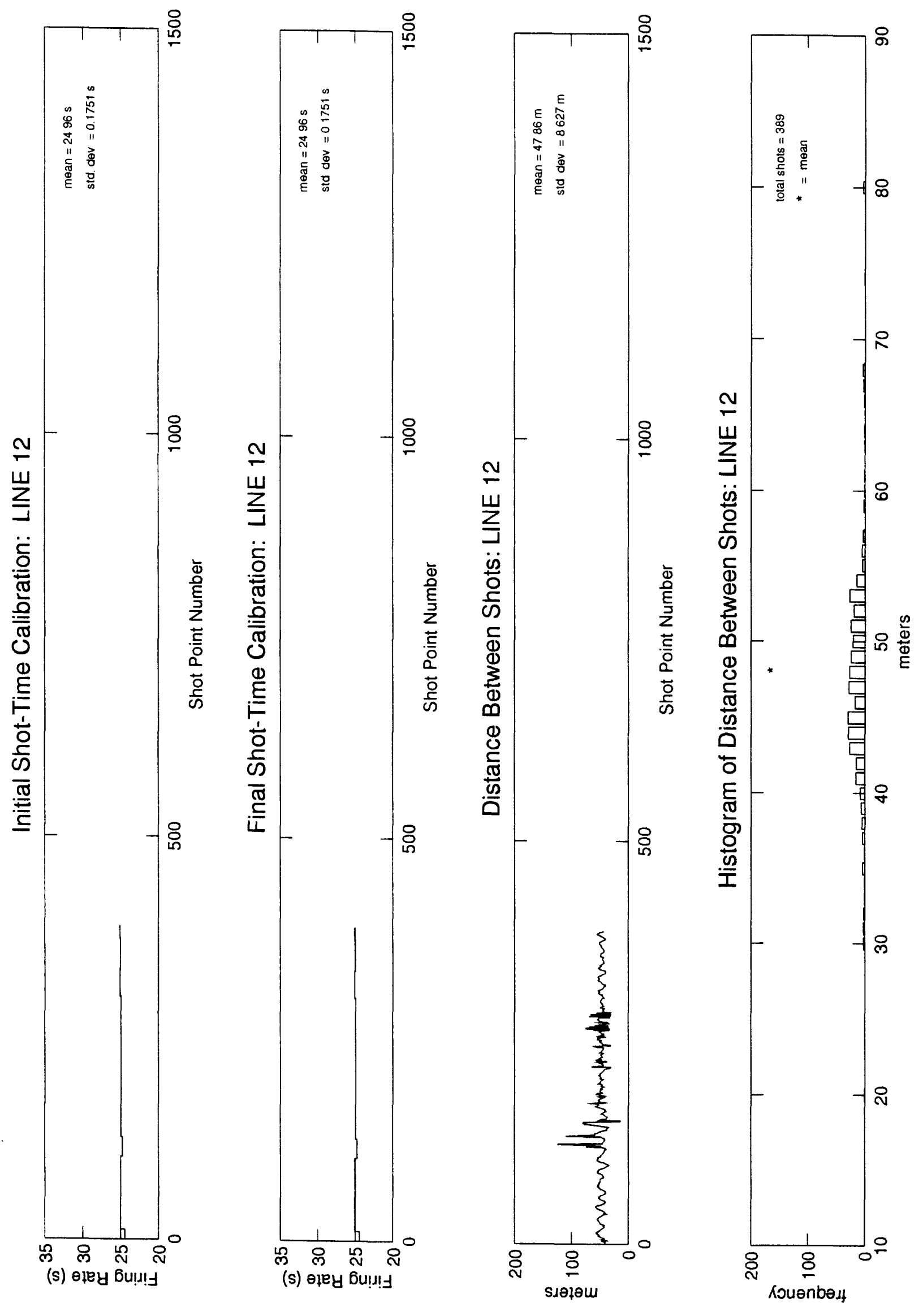




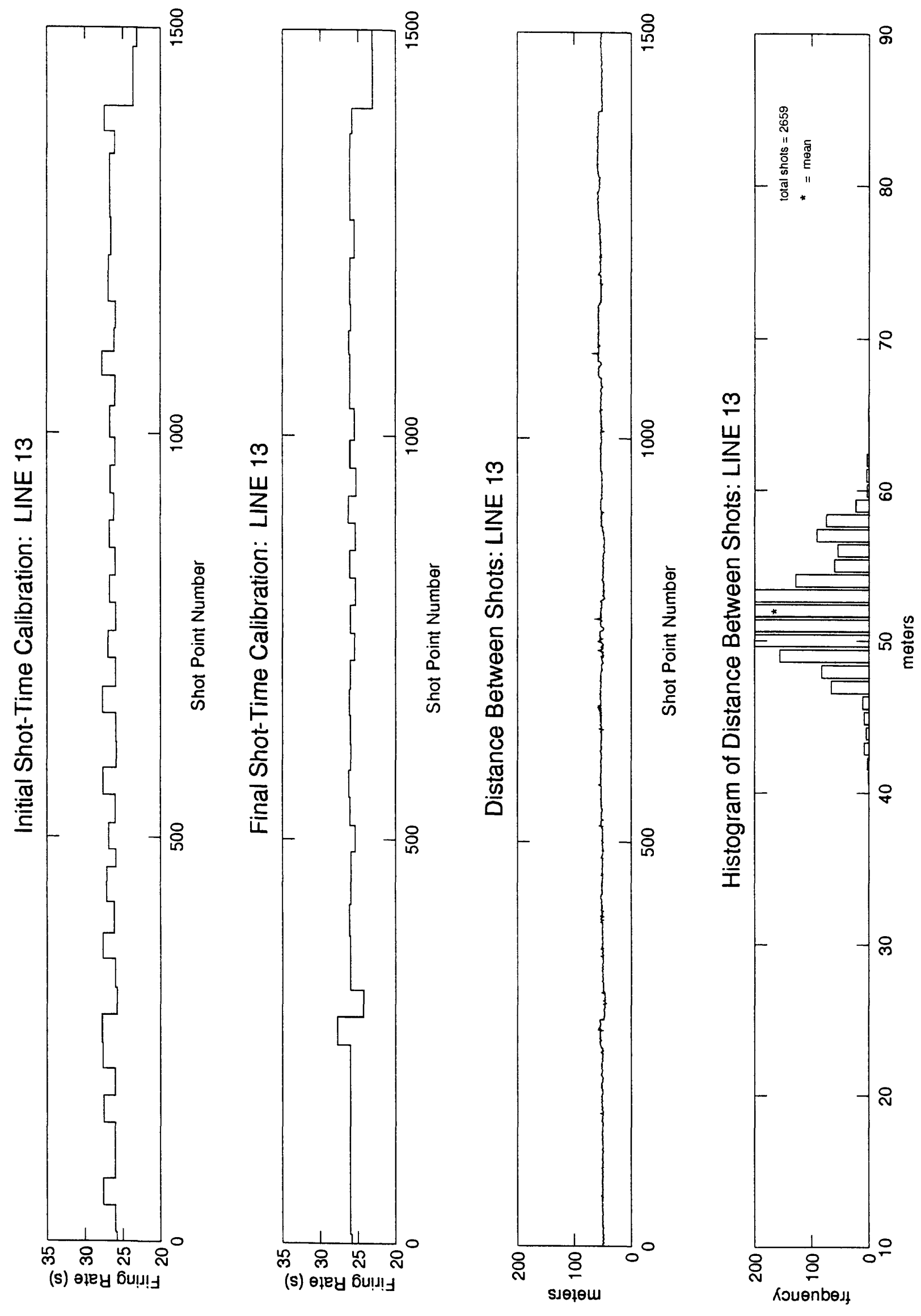



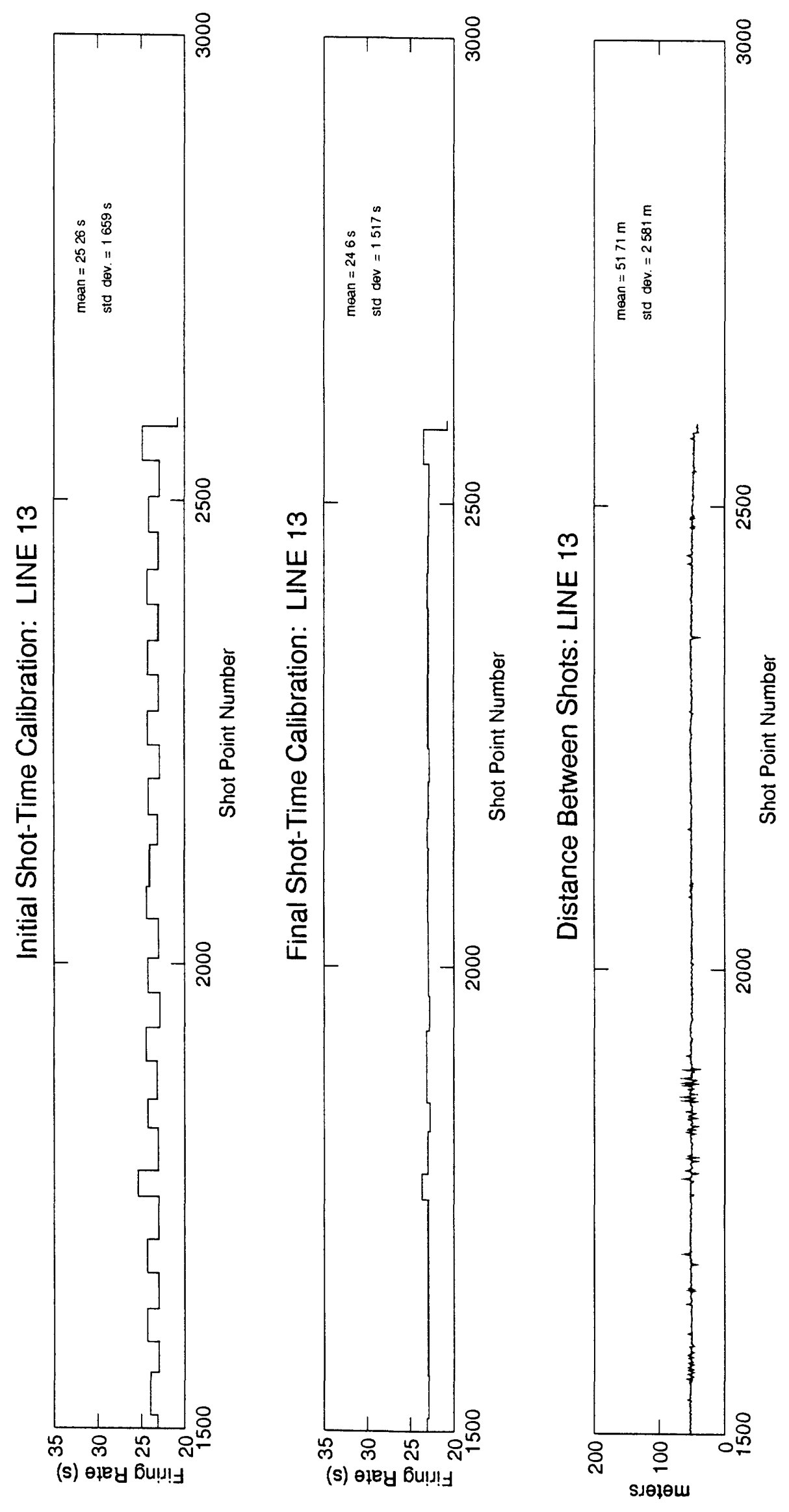


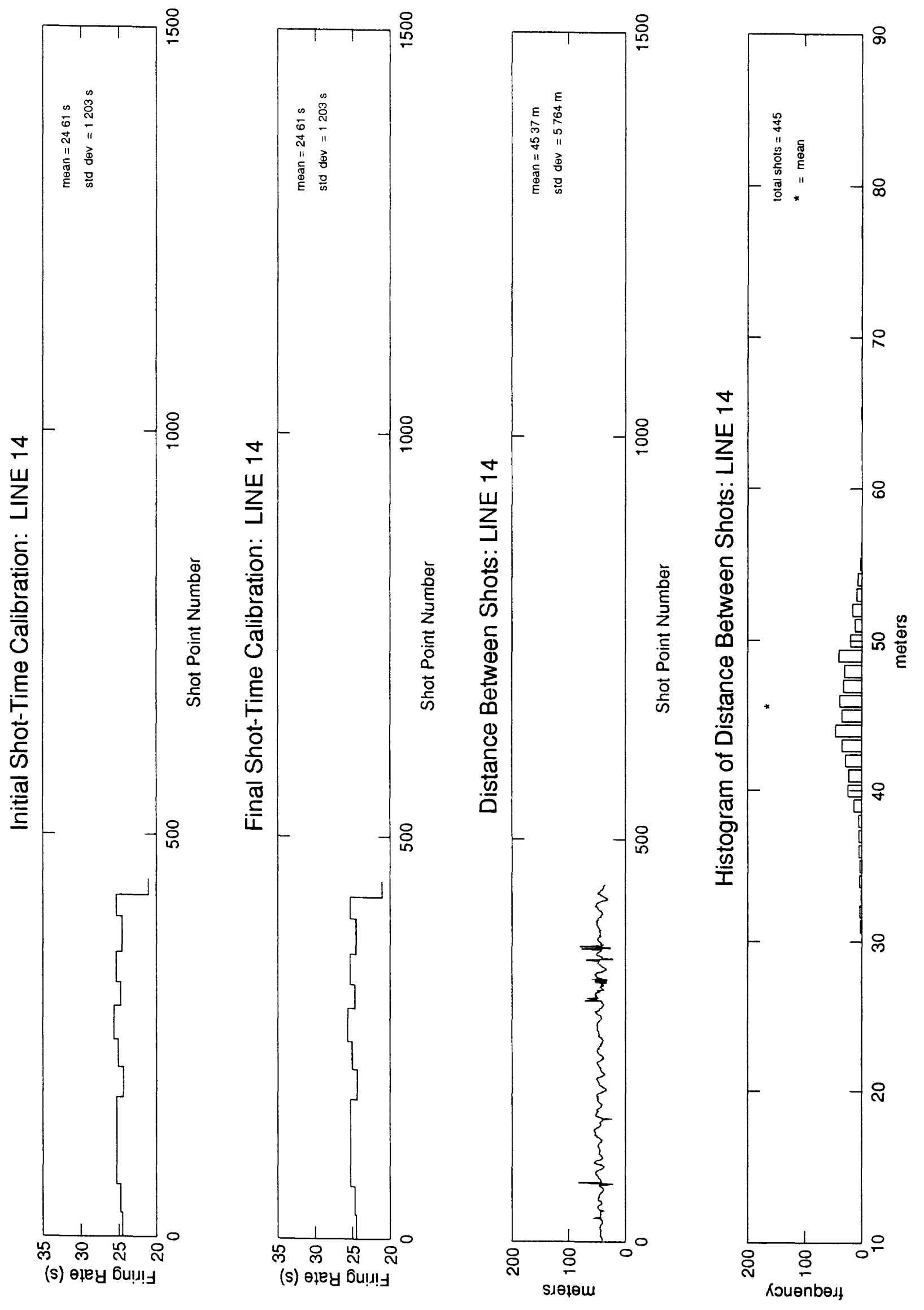




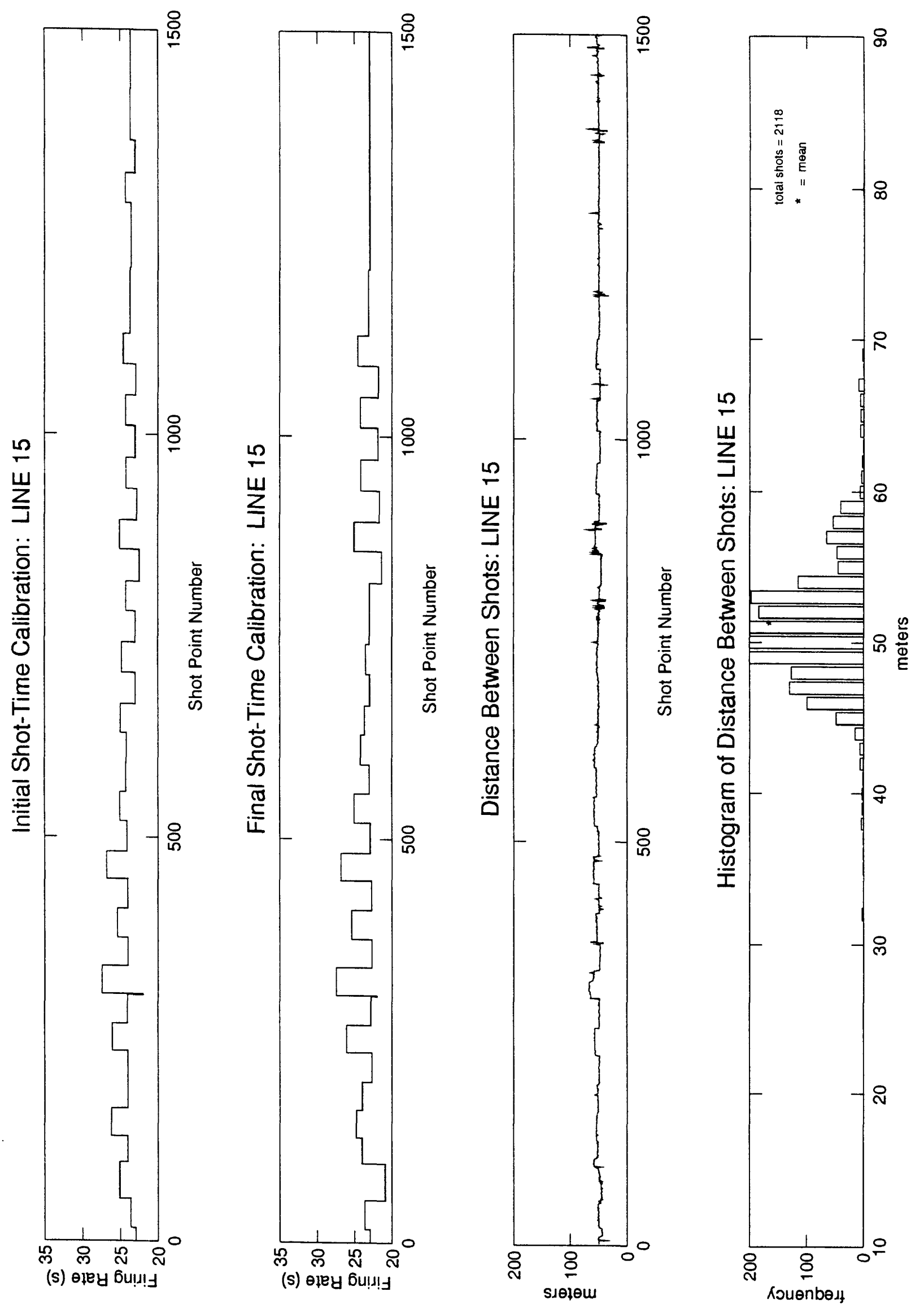



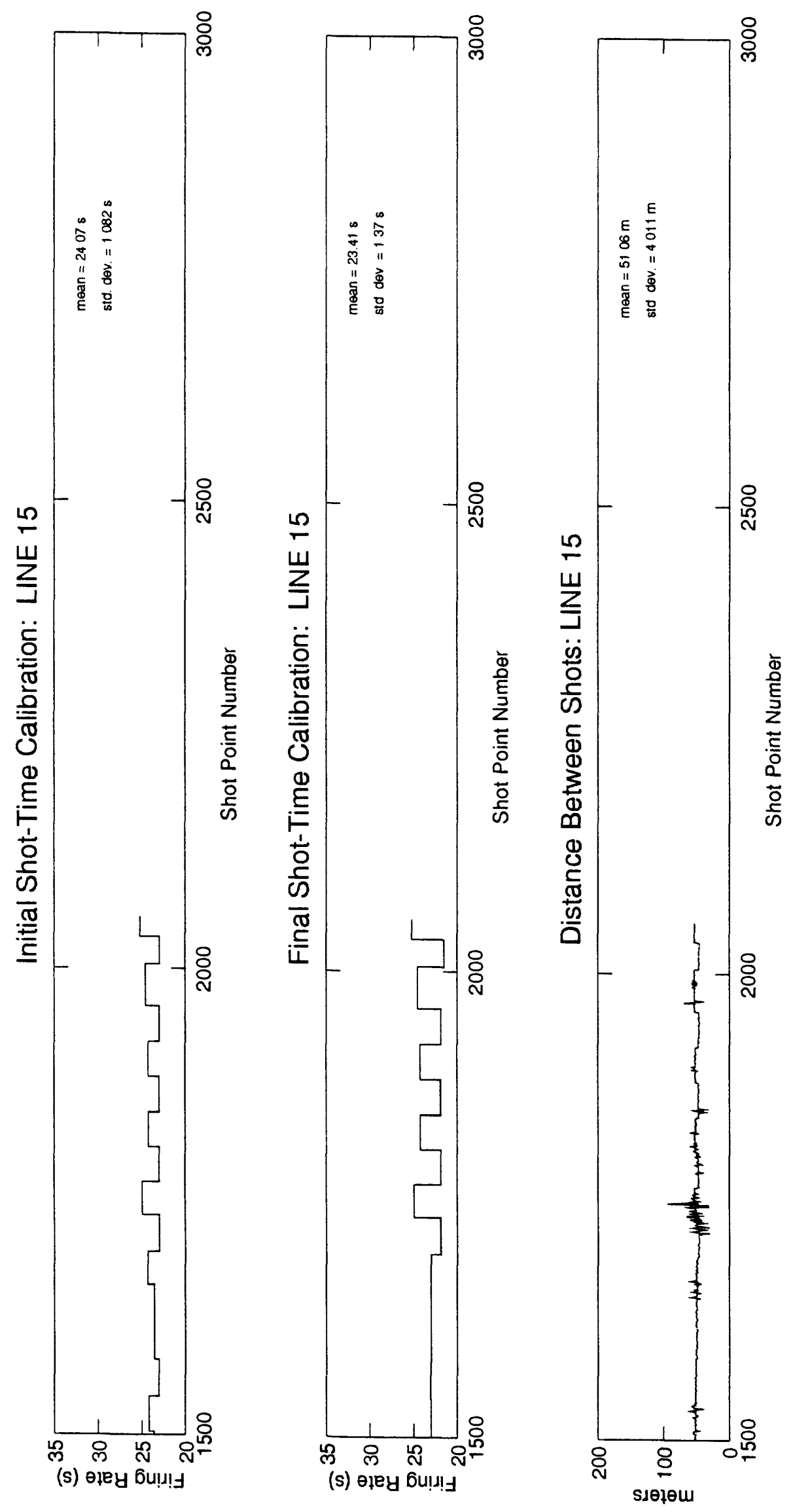


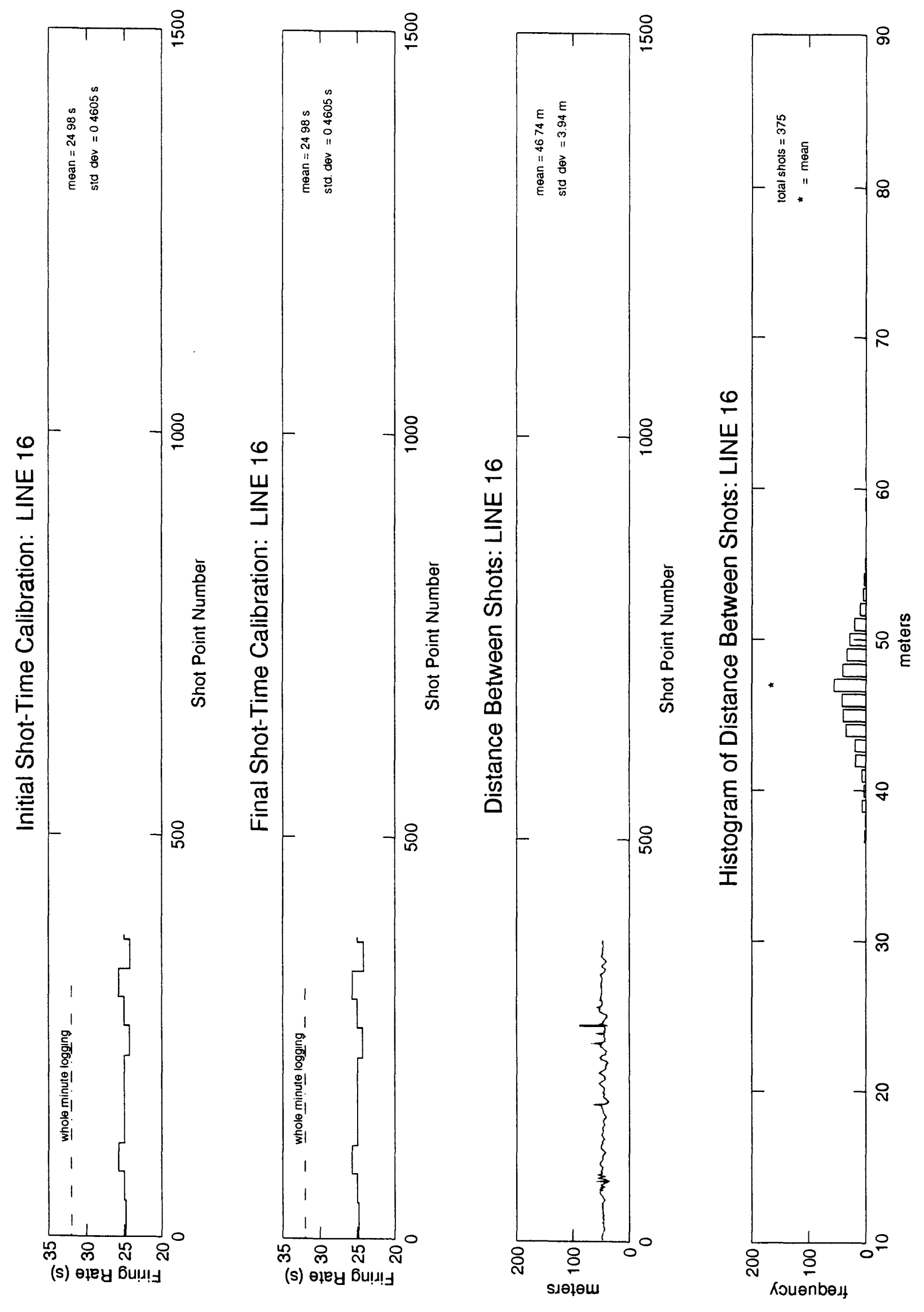




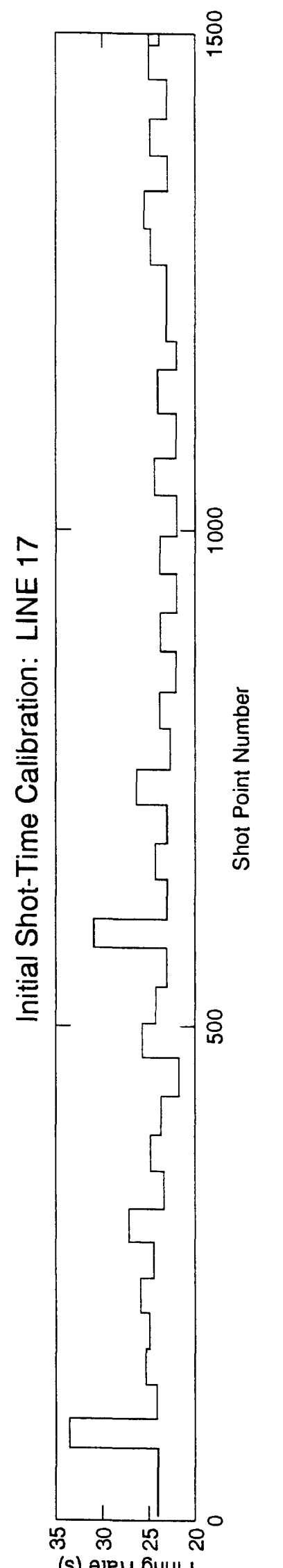

(s) өley 6u!l!
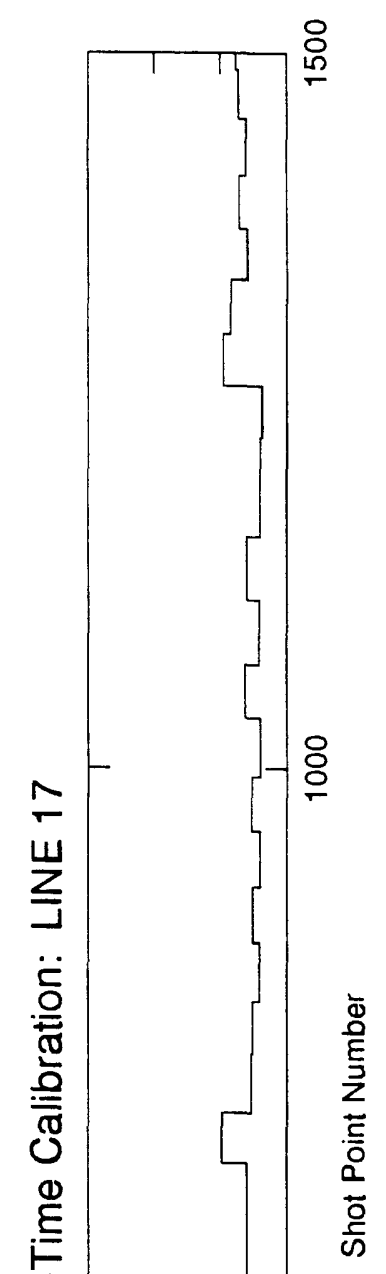

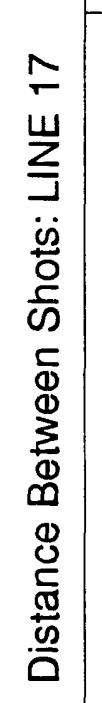

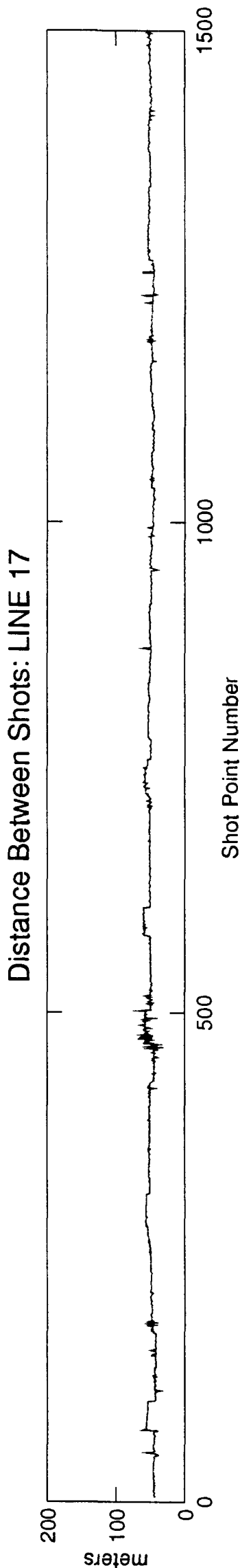

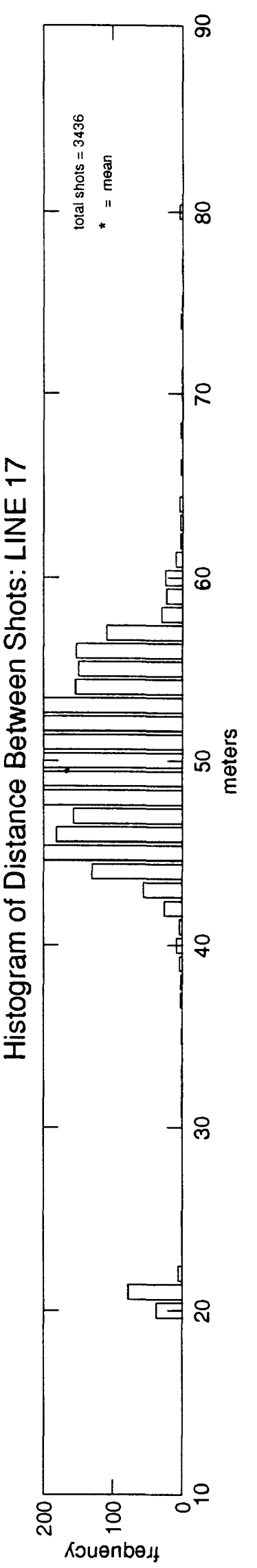




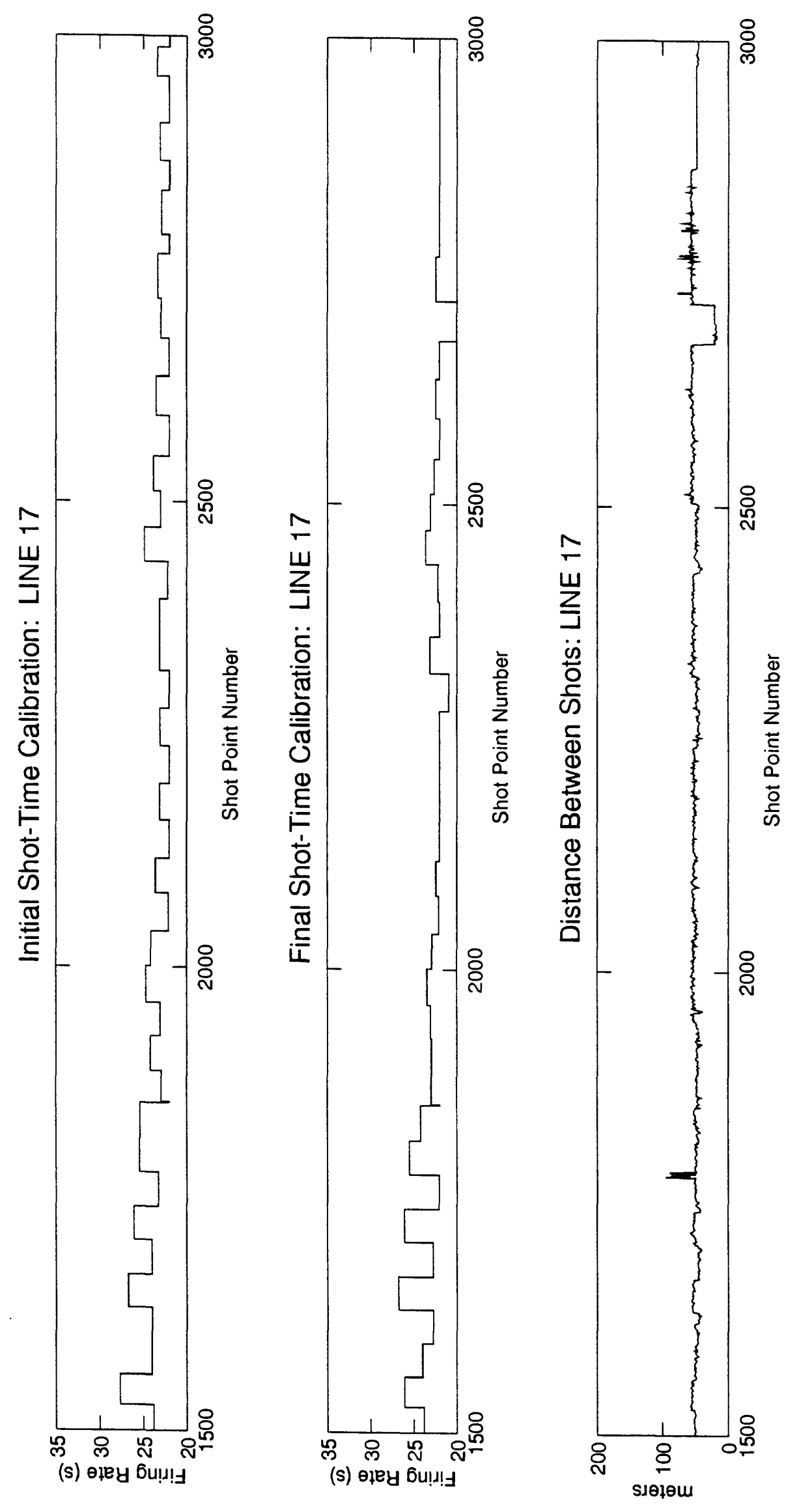



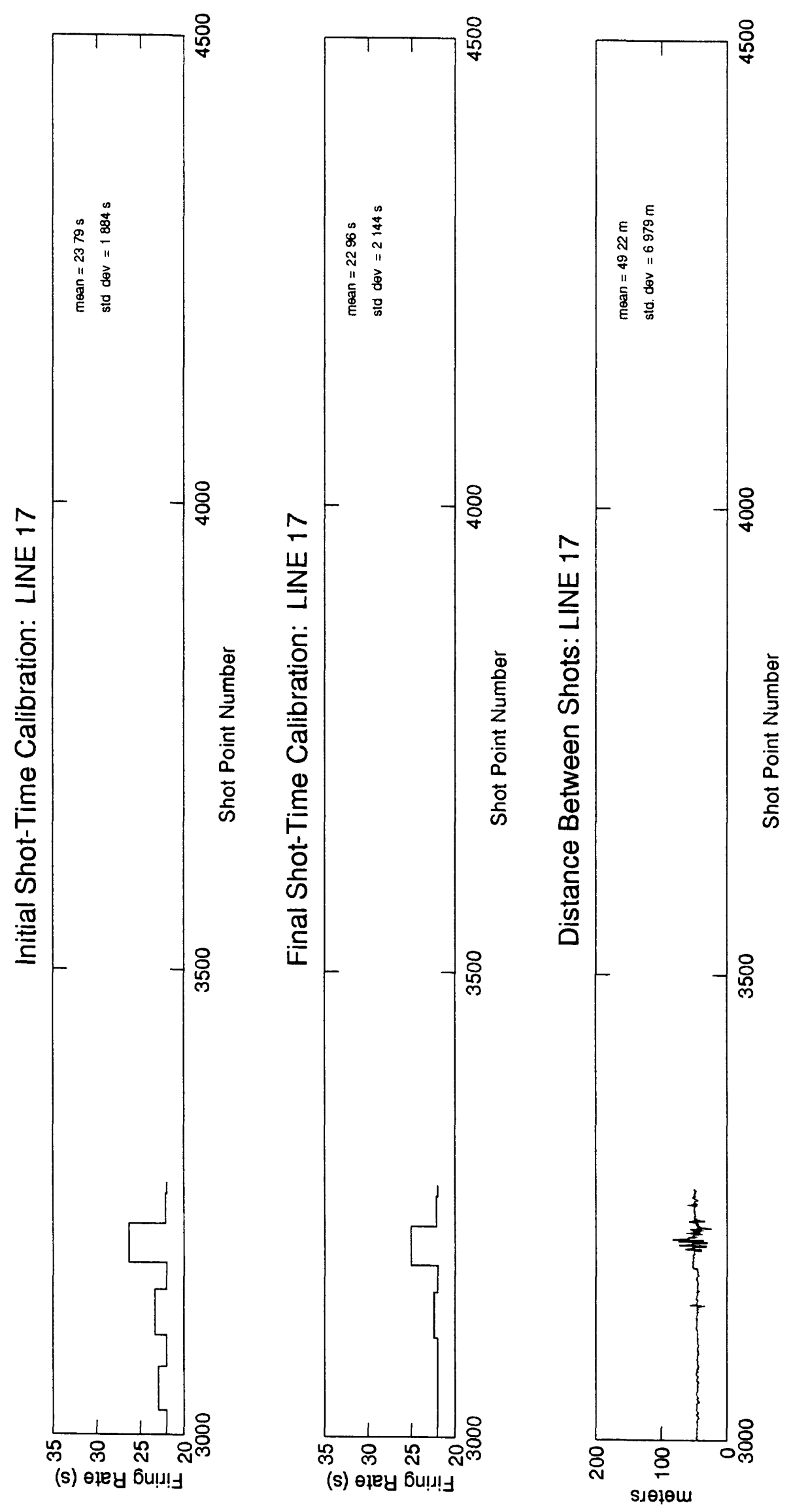


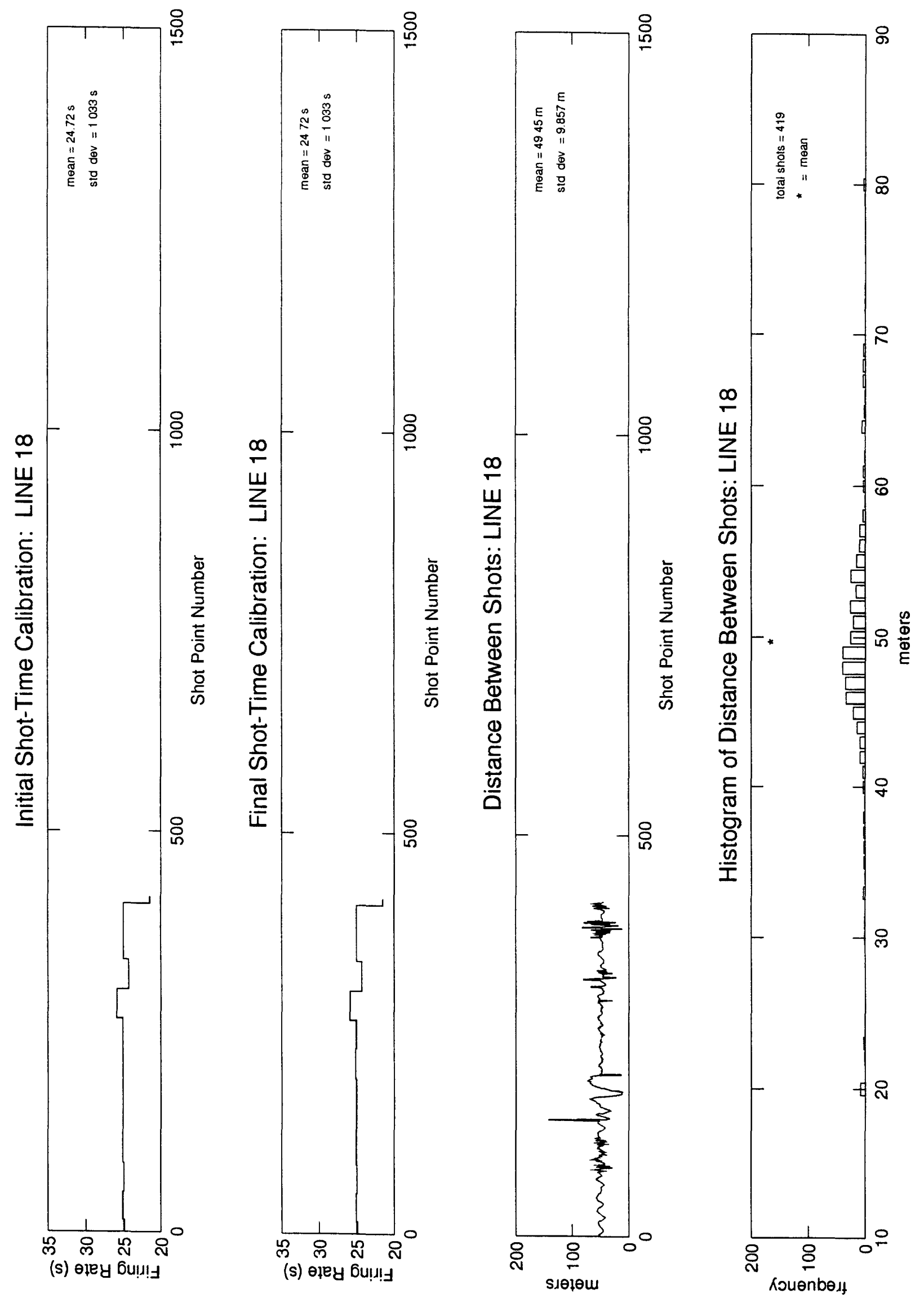




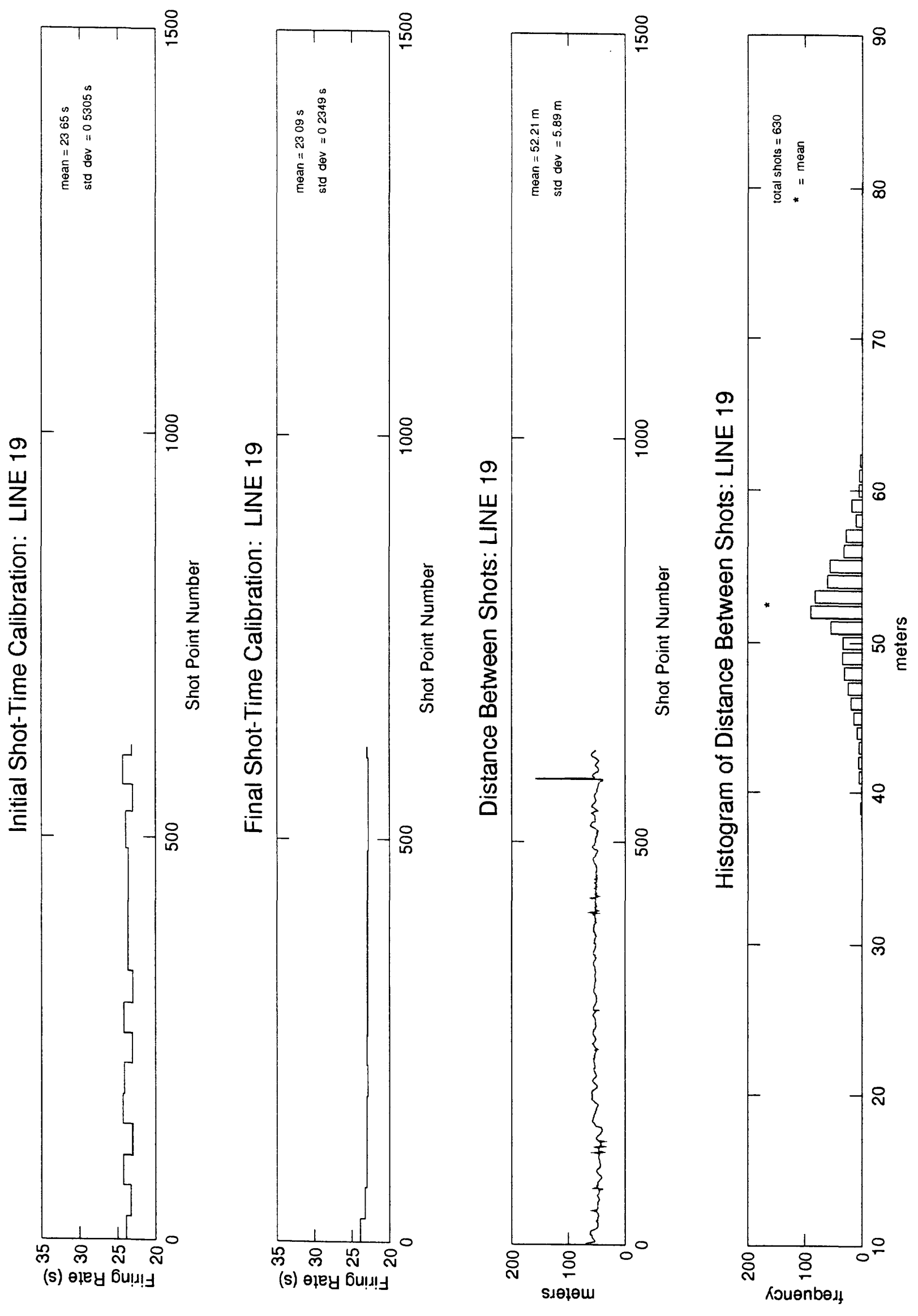



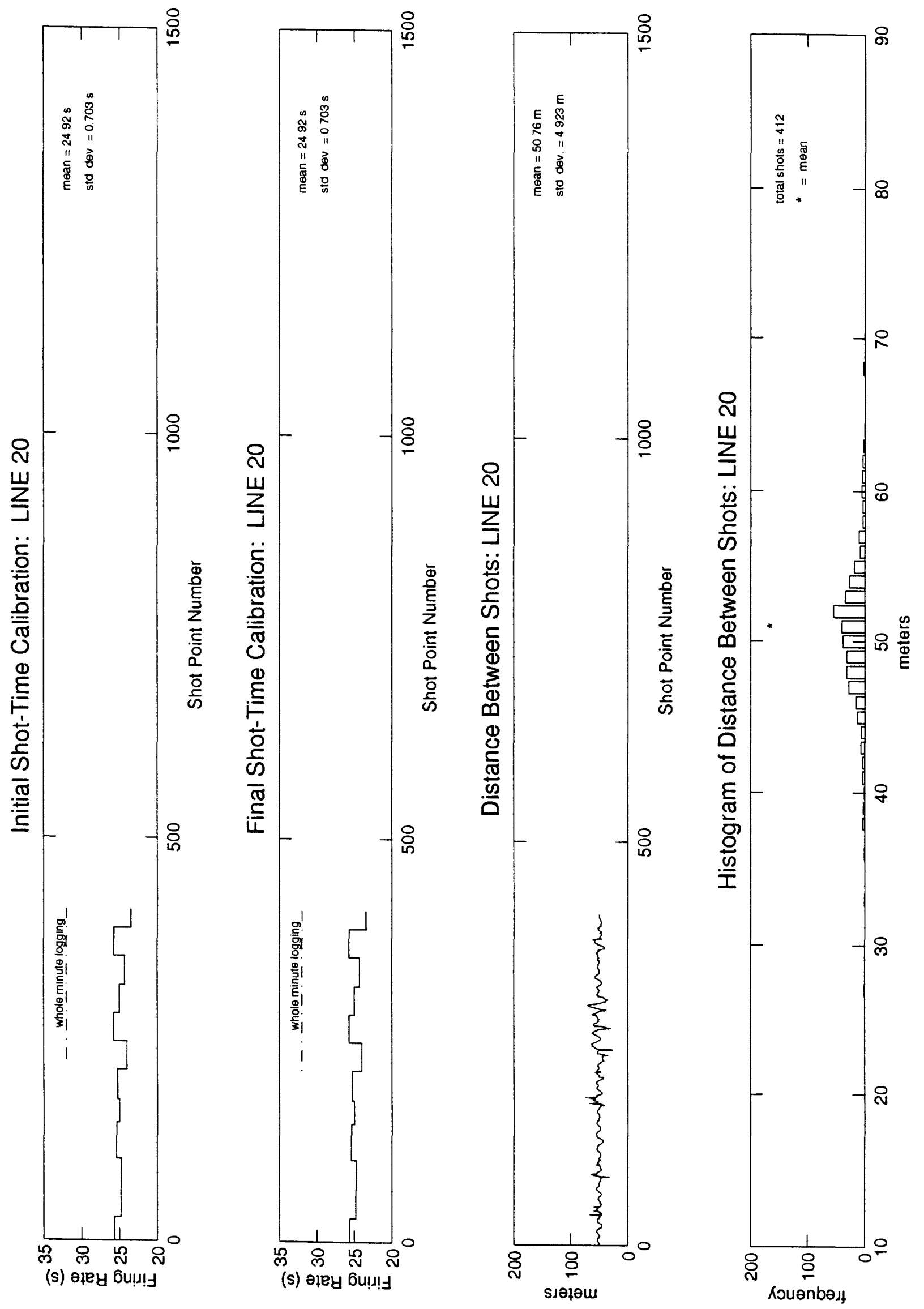

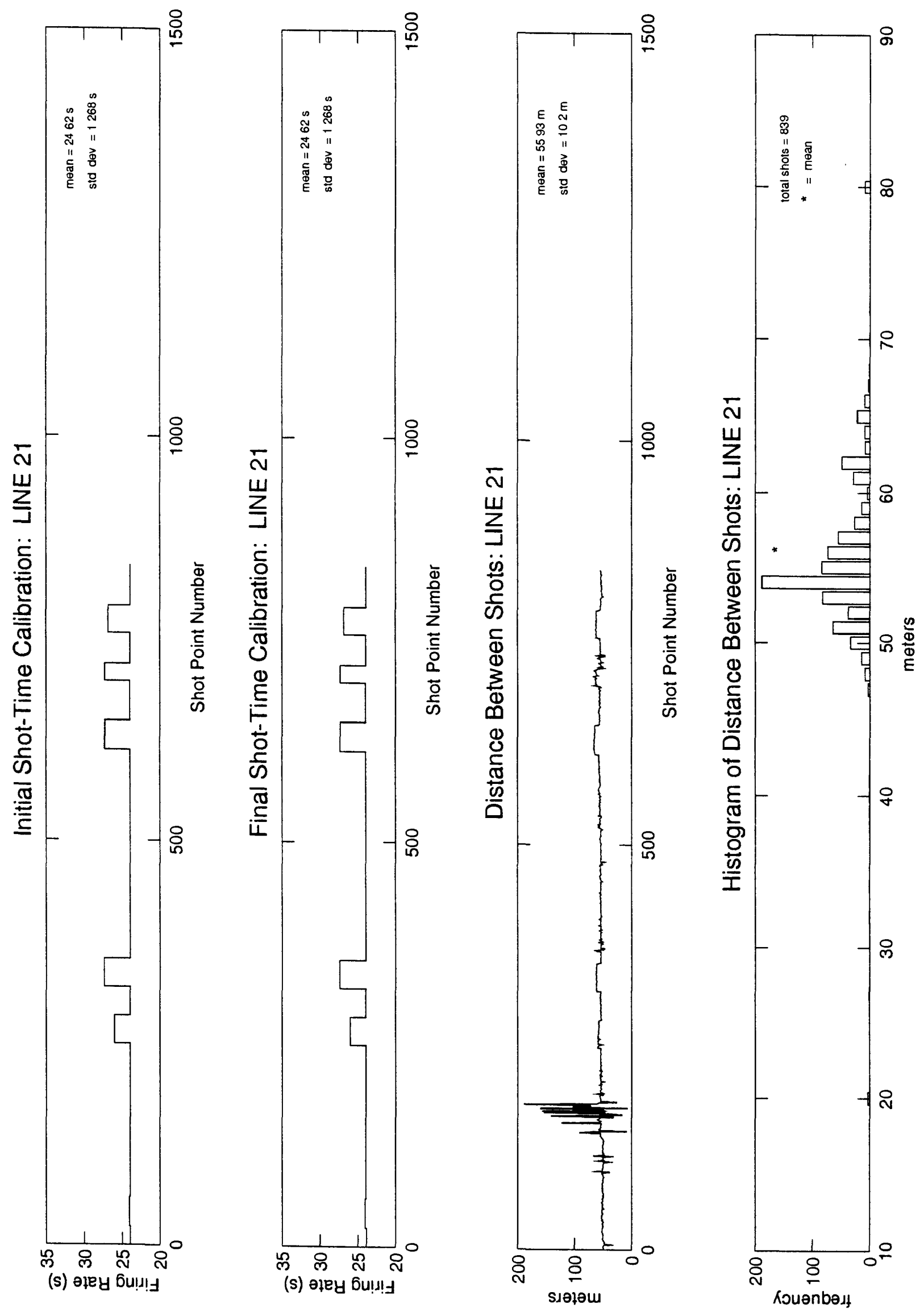


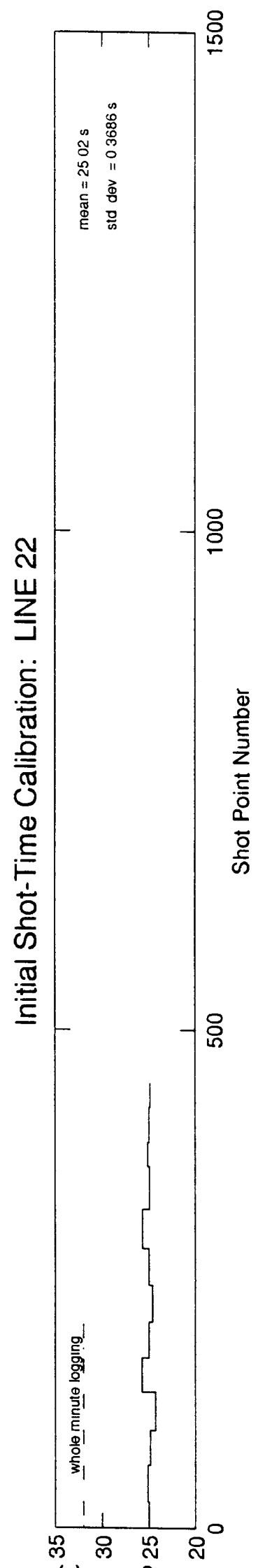

(s) өाey Gu!n!y
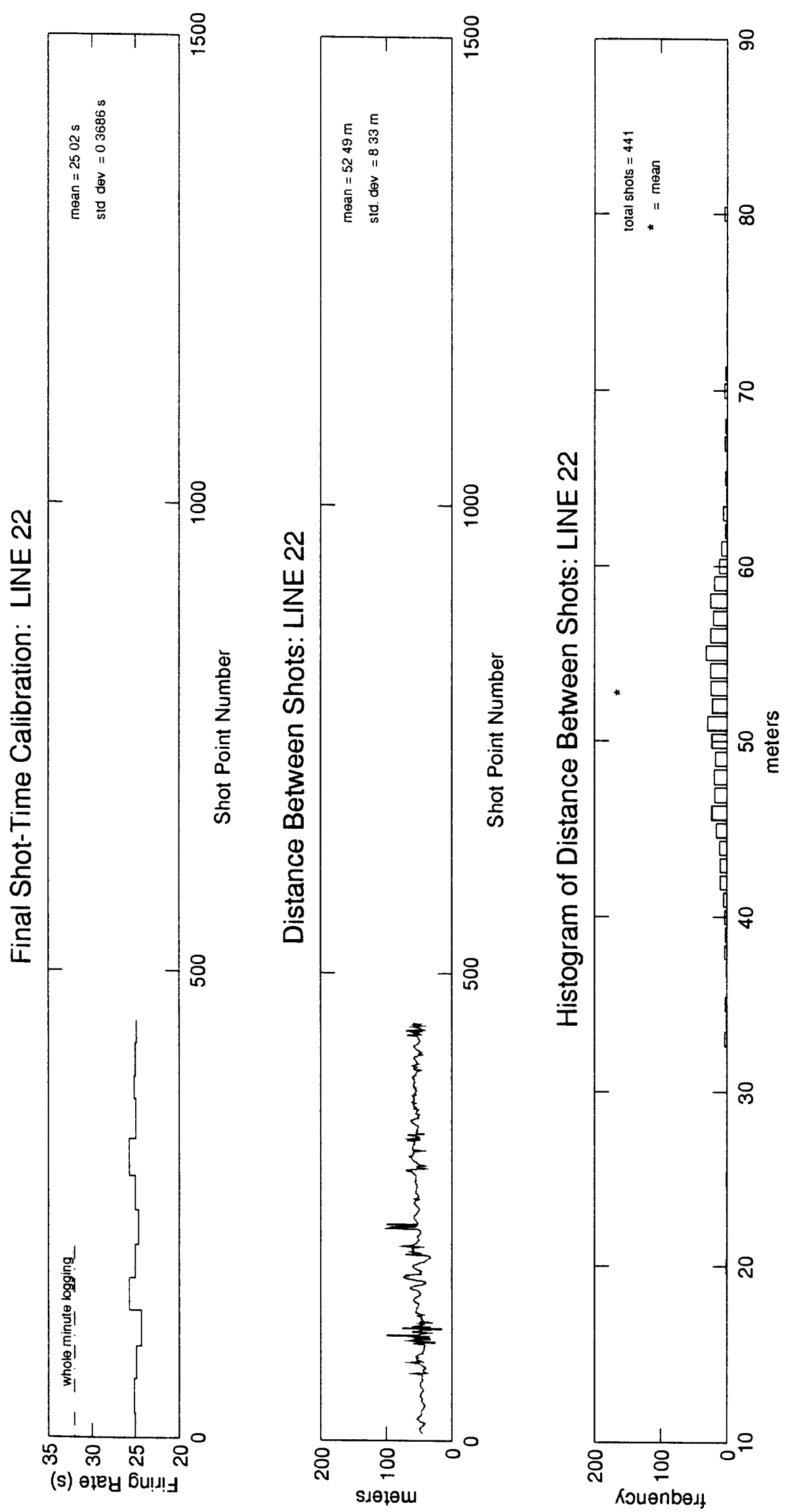

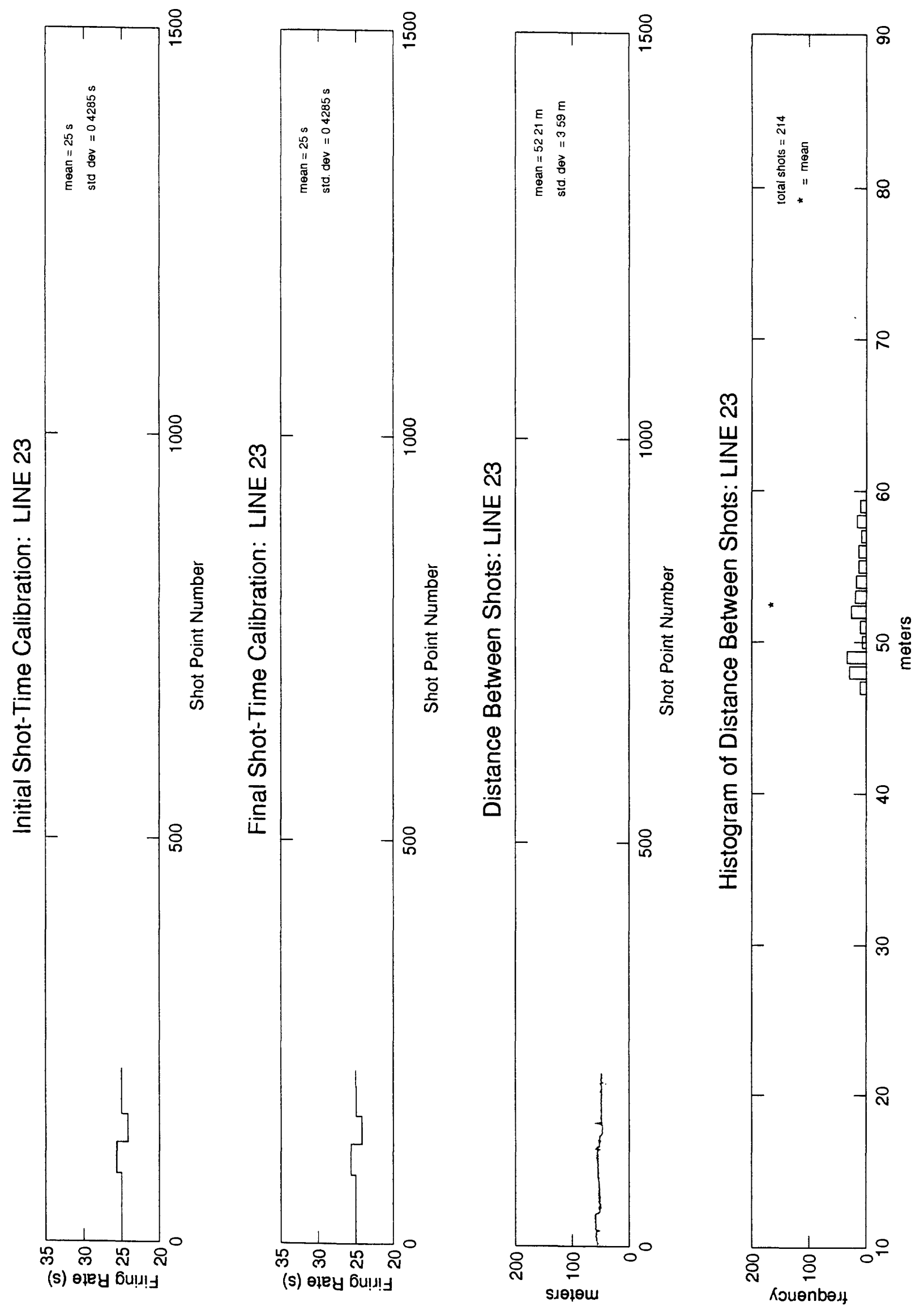


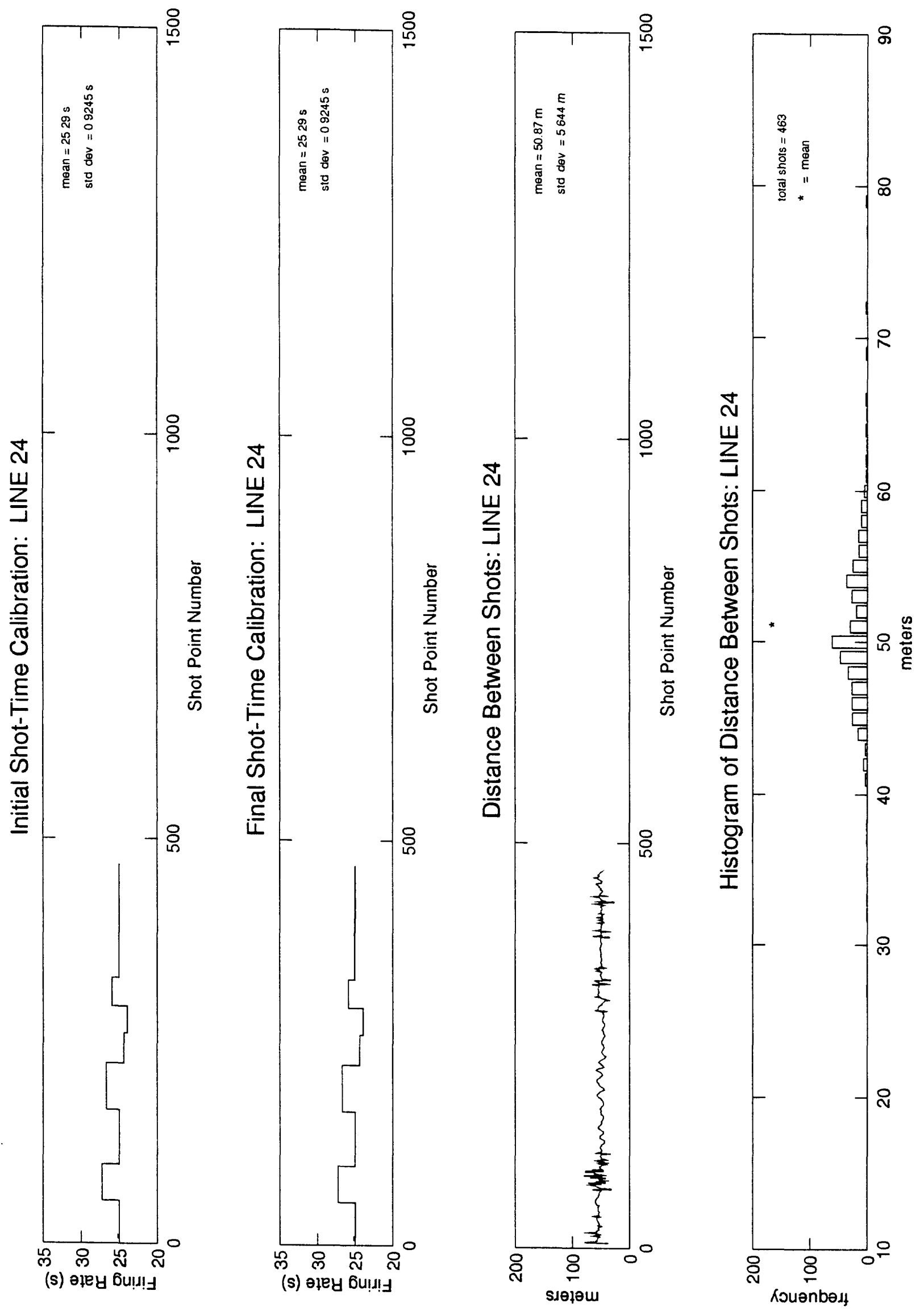




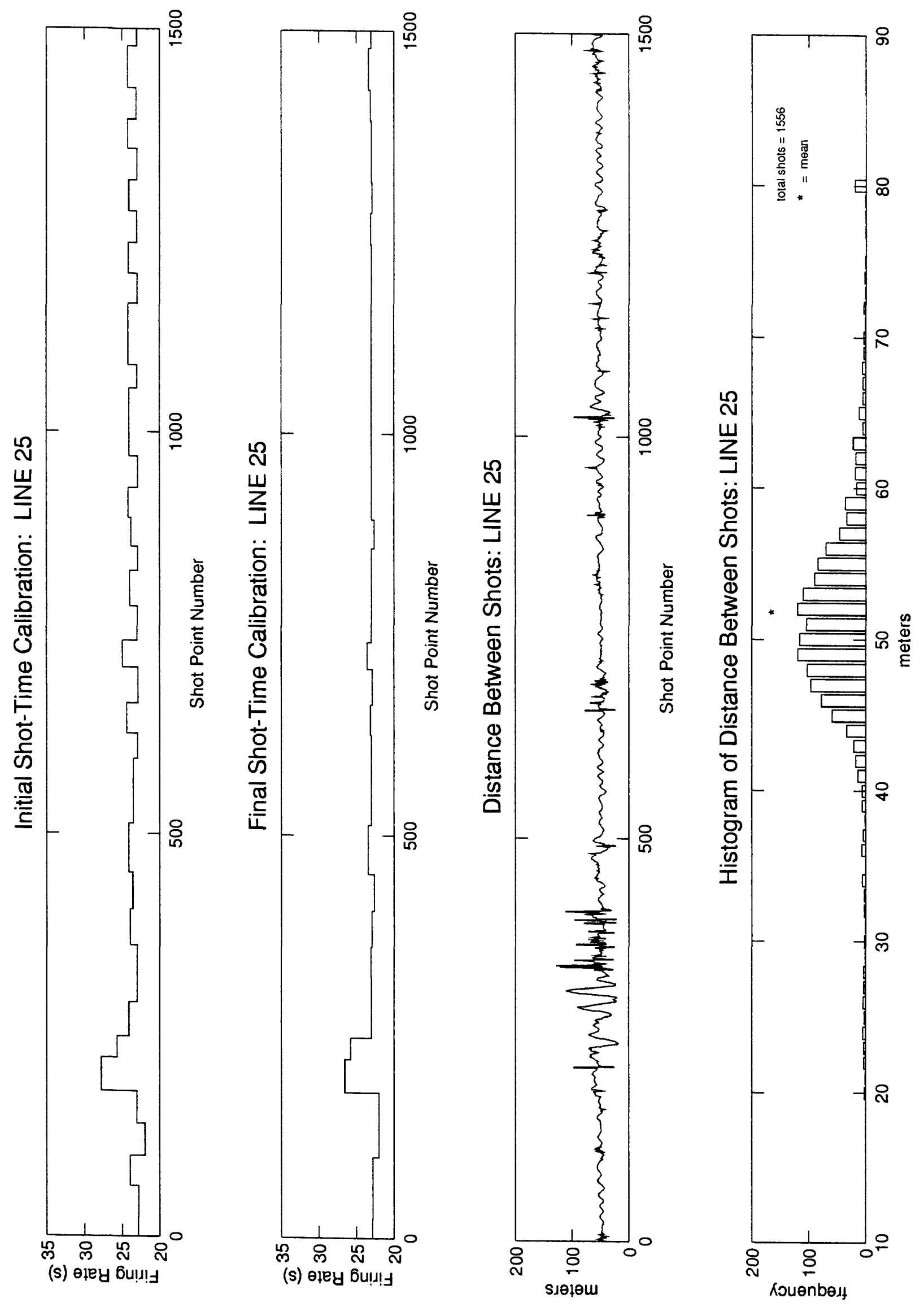



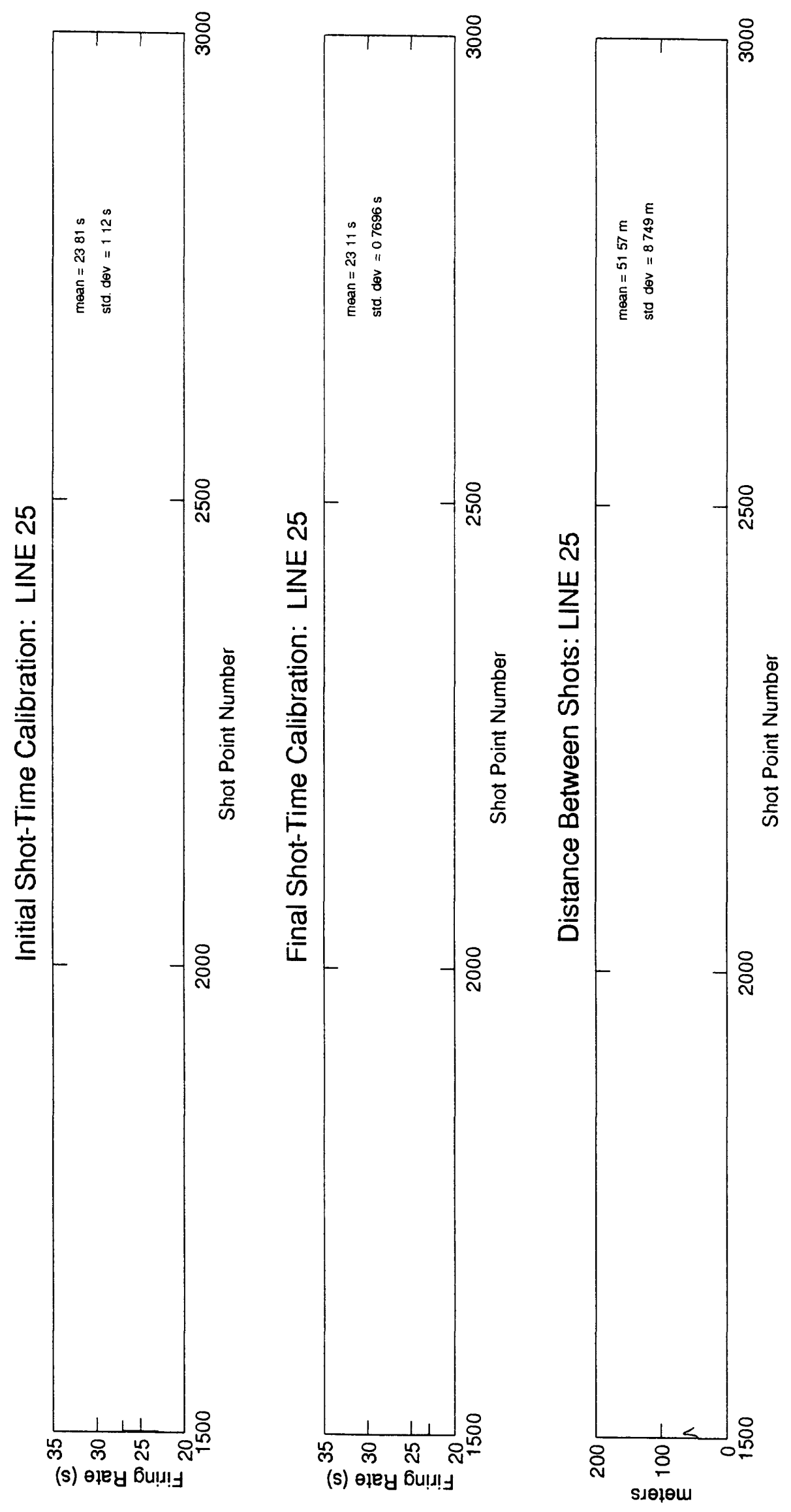


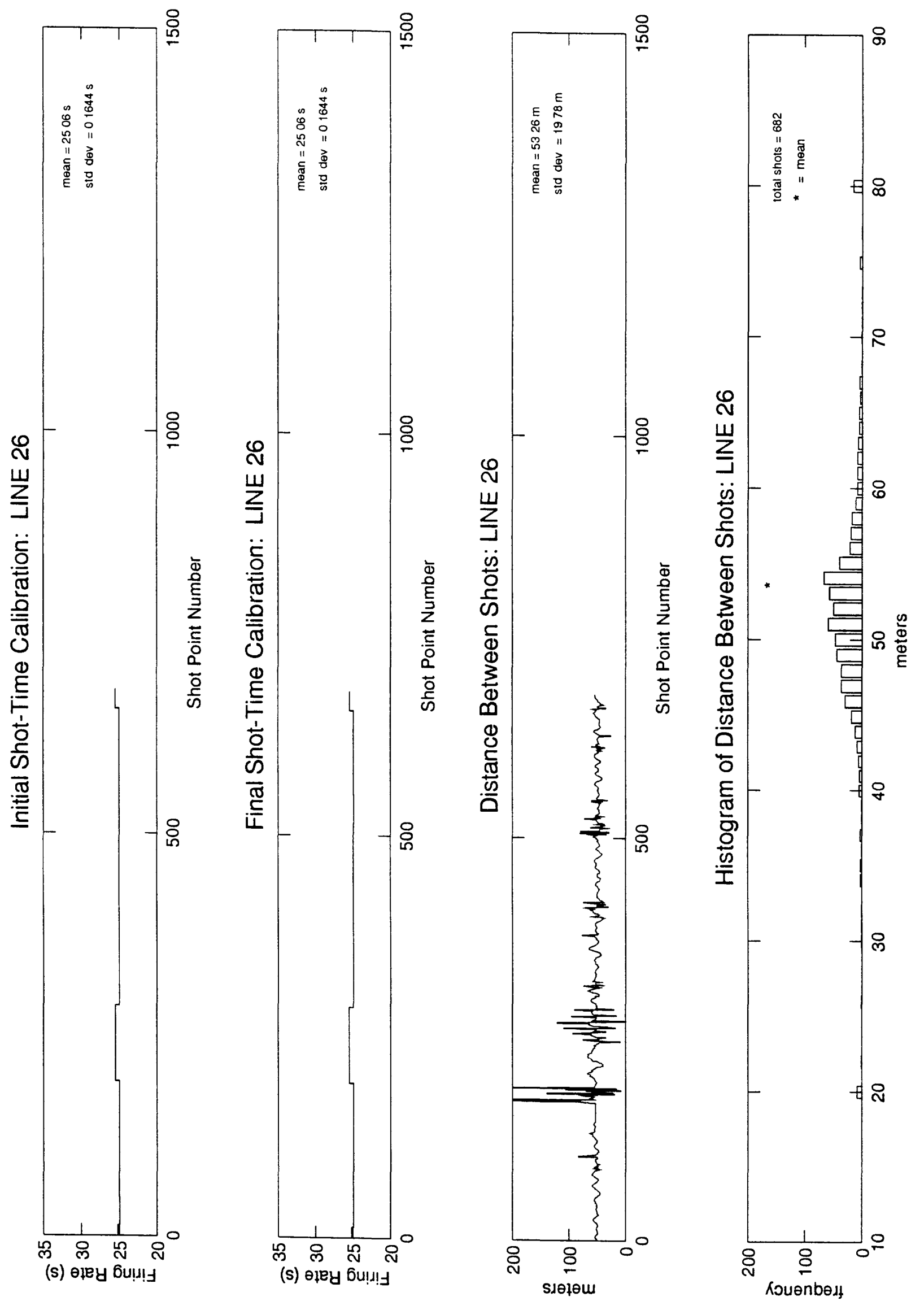




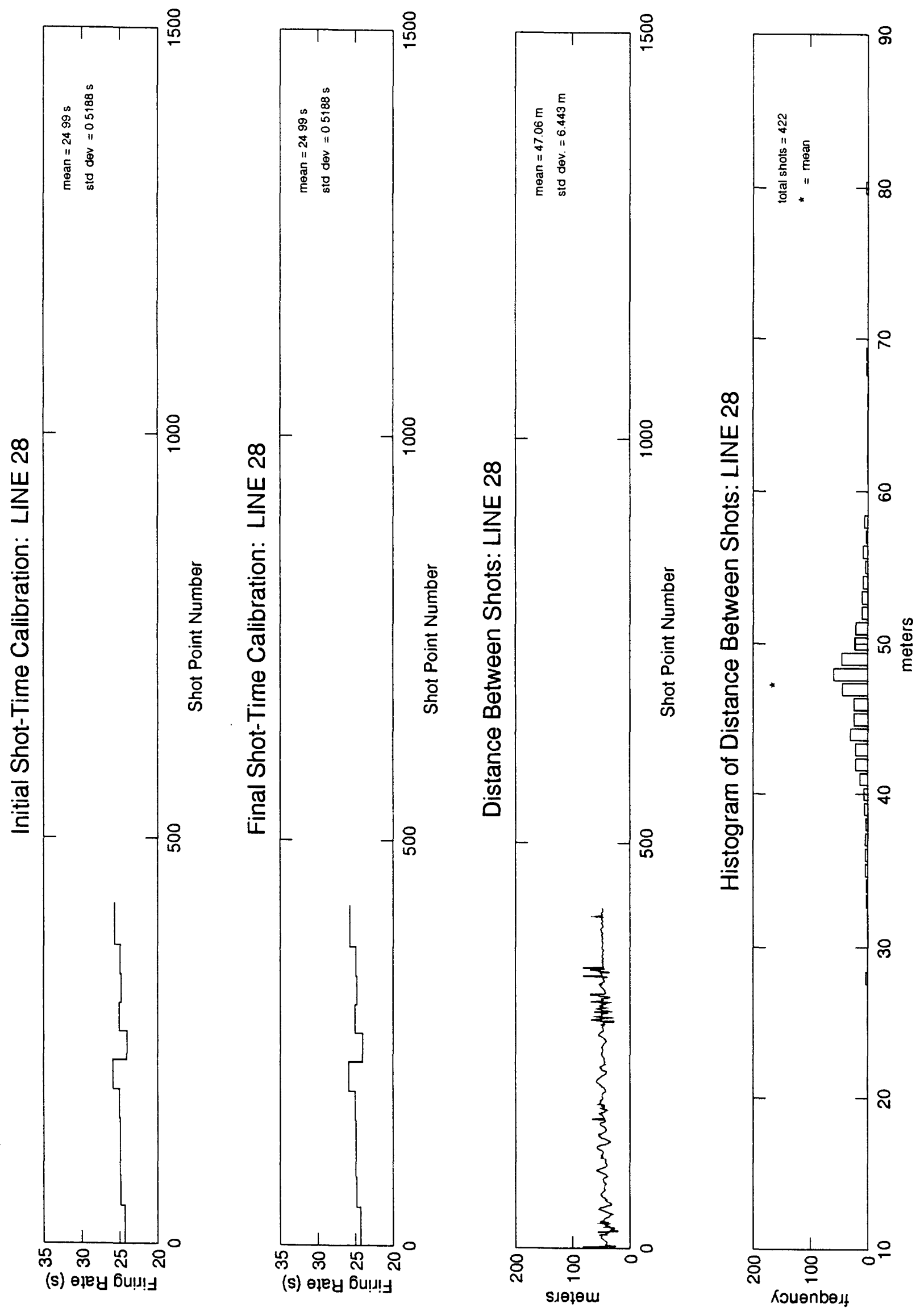



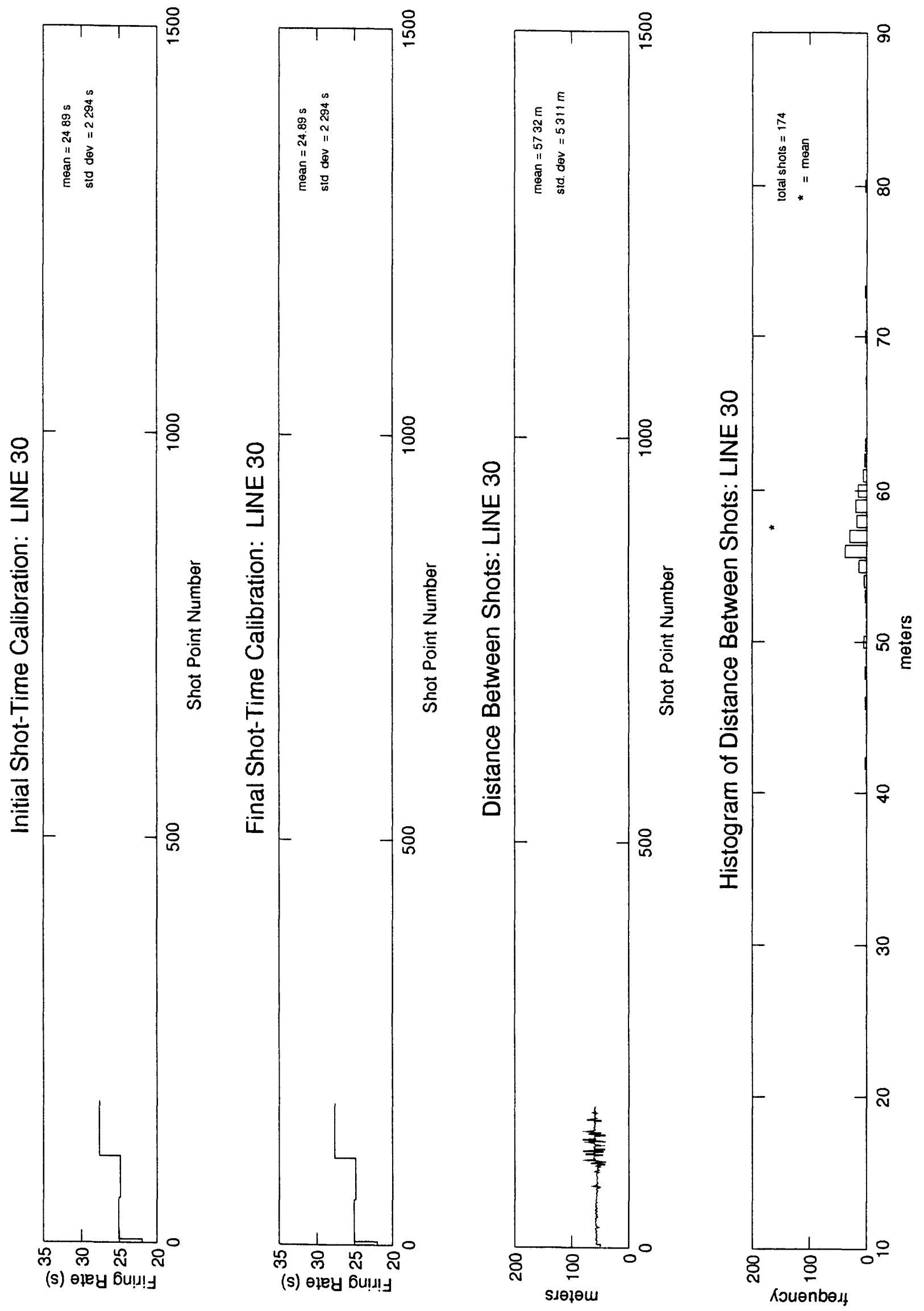

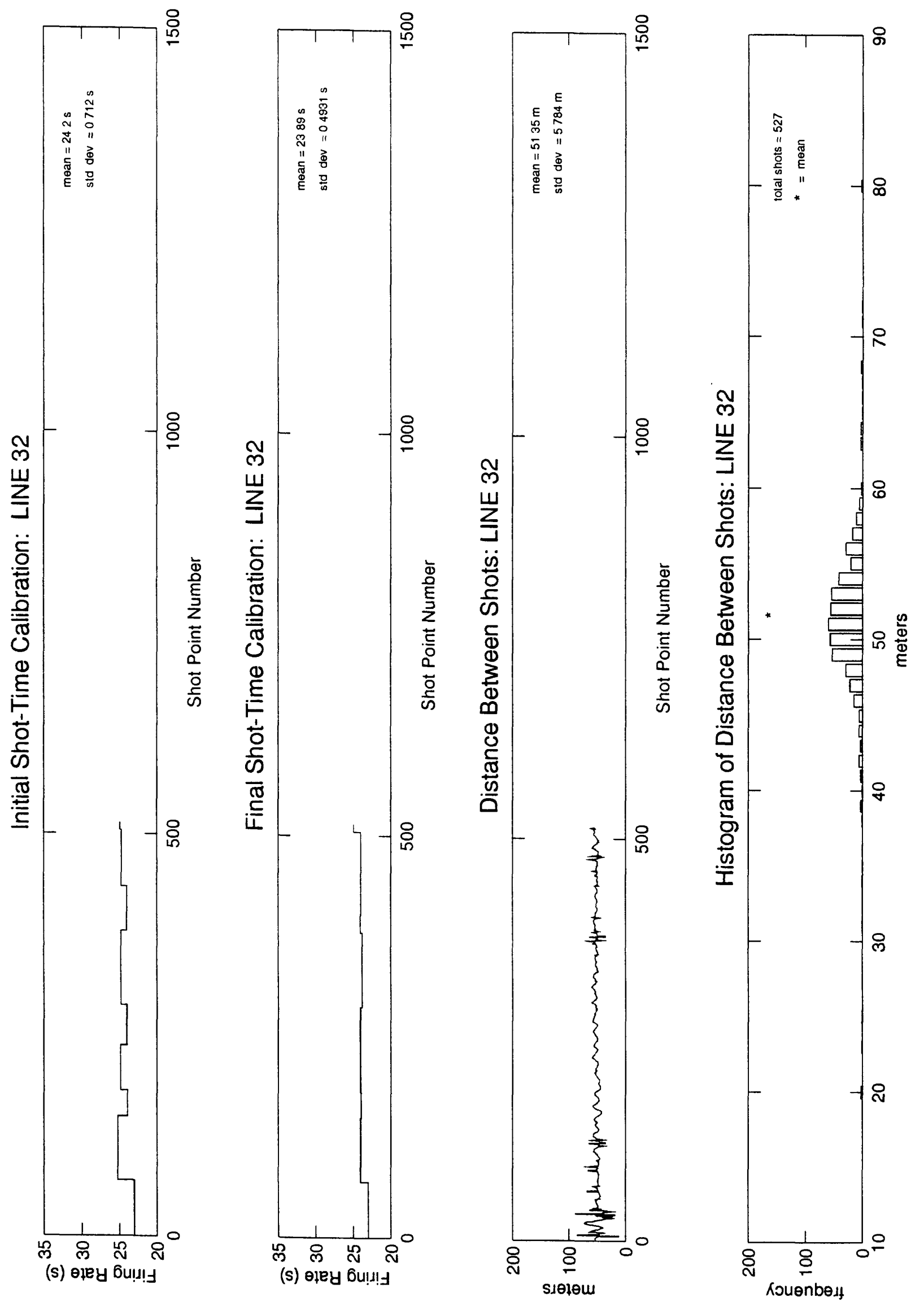

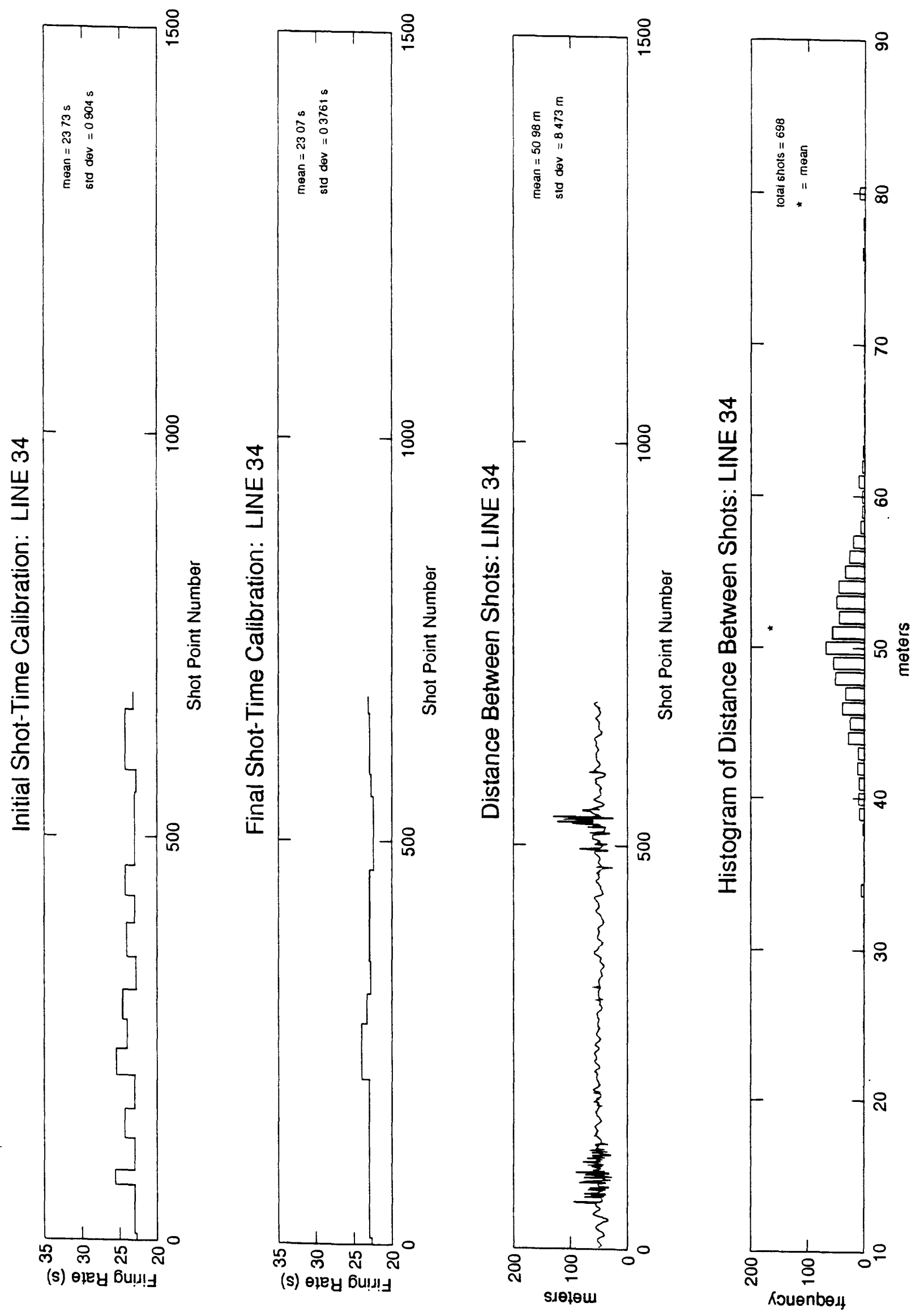

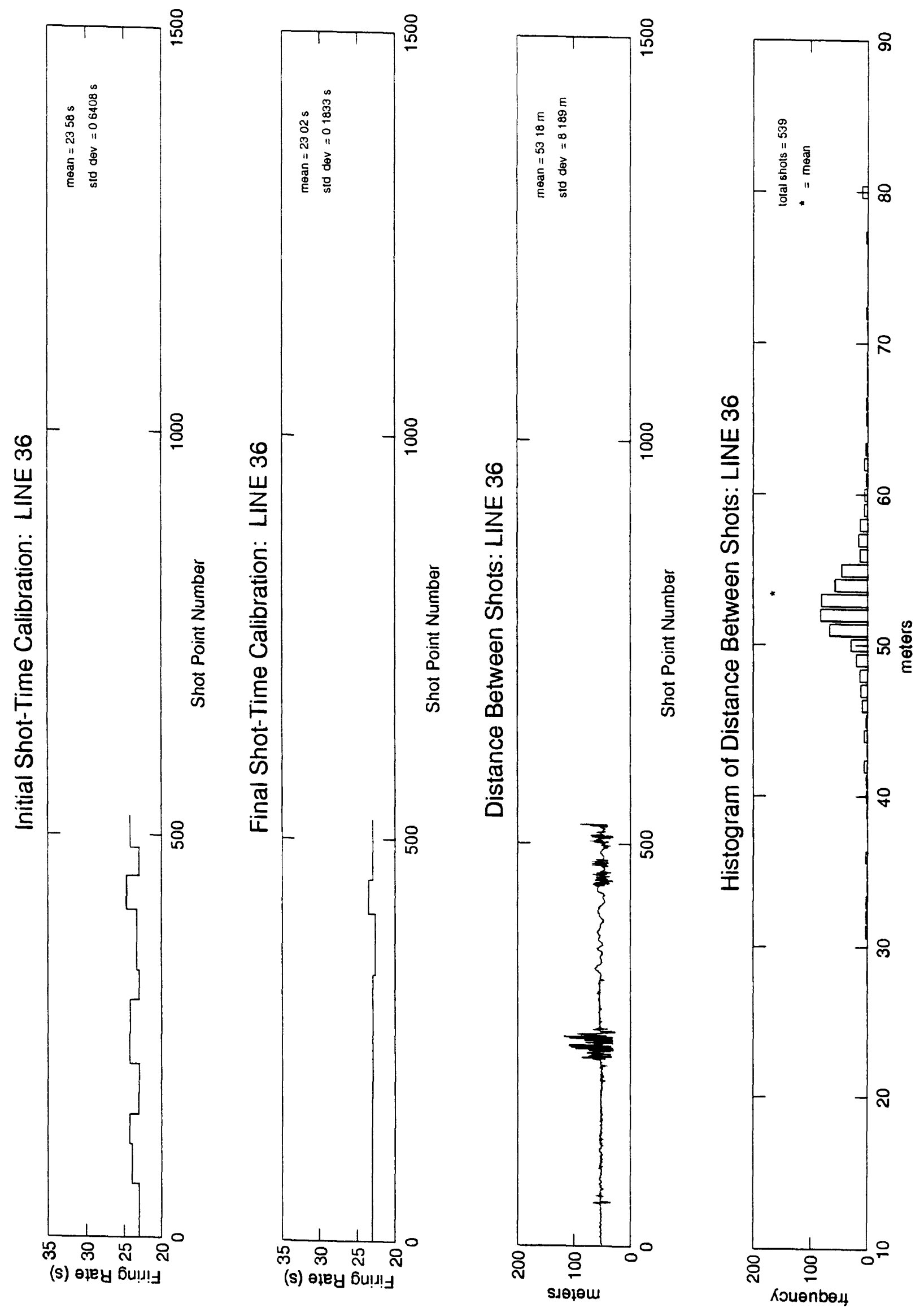

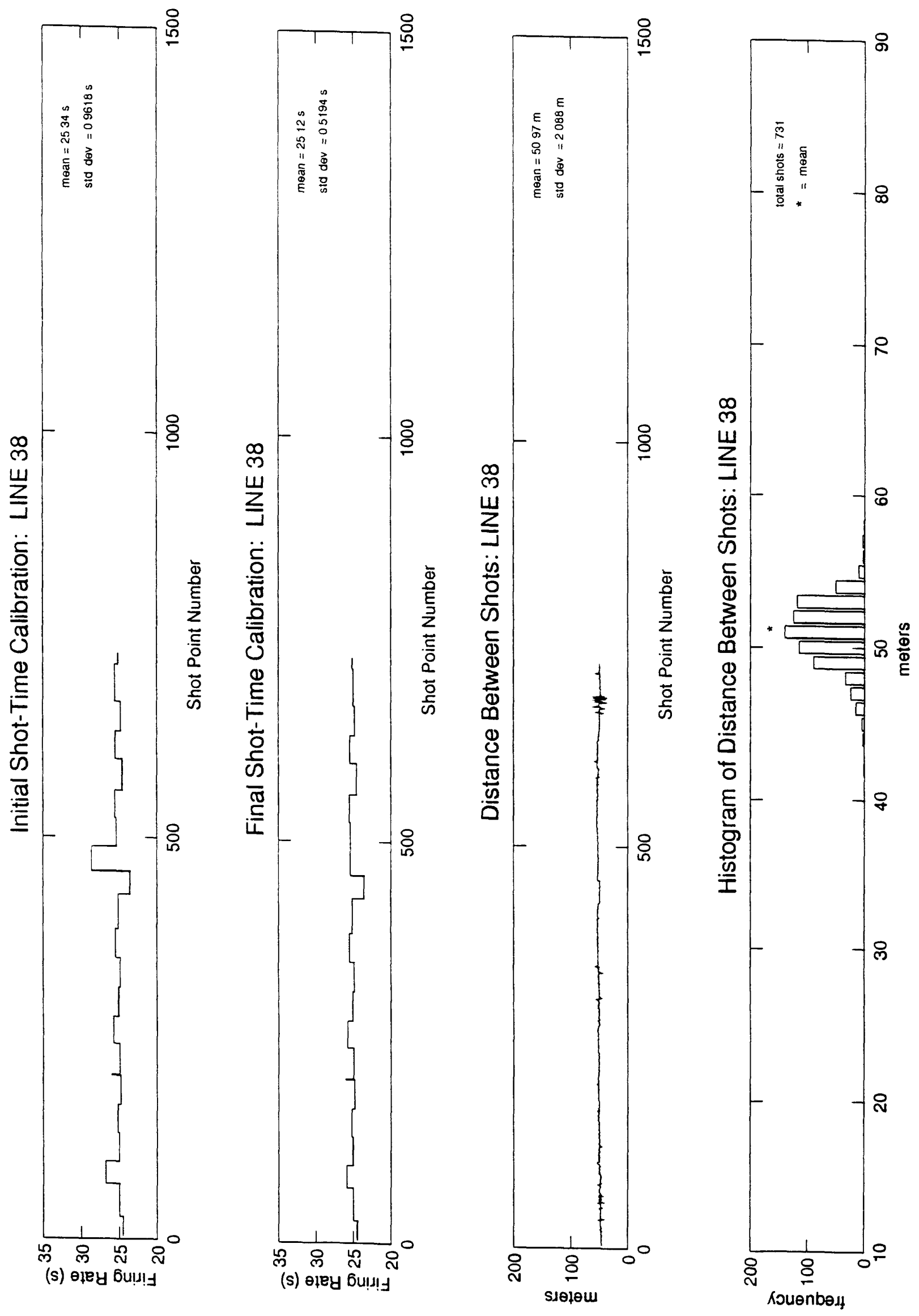


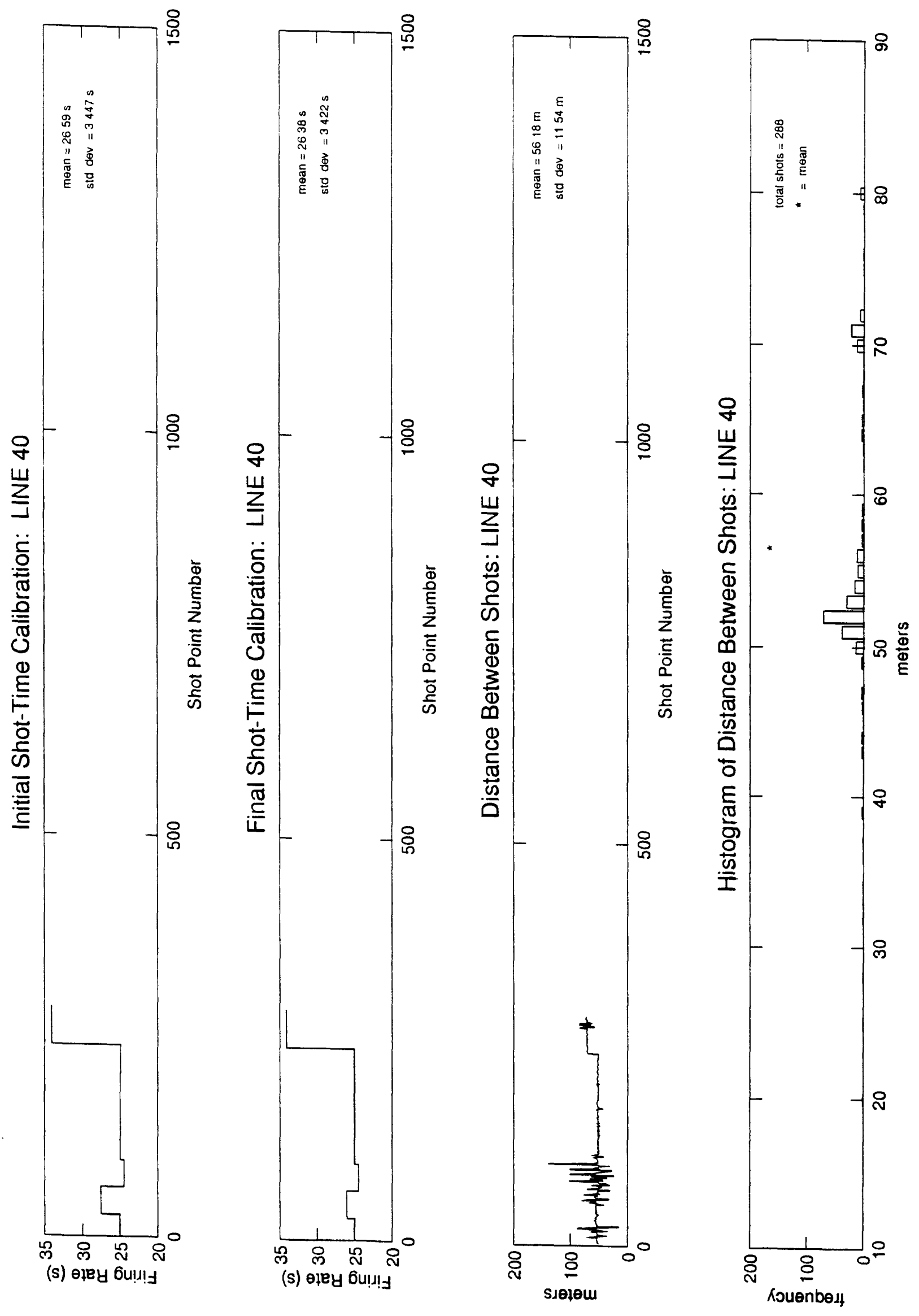



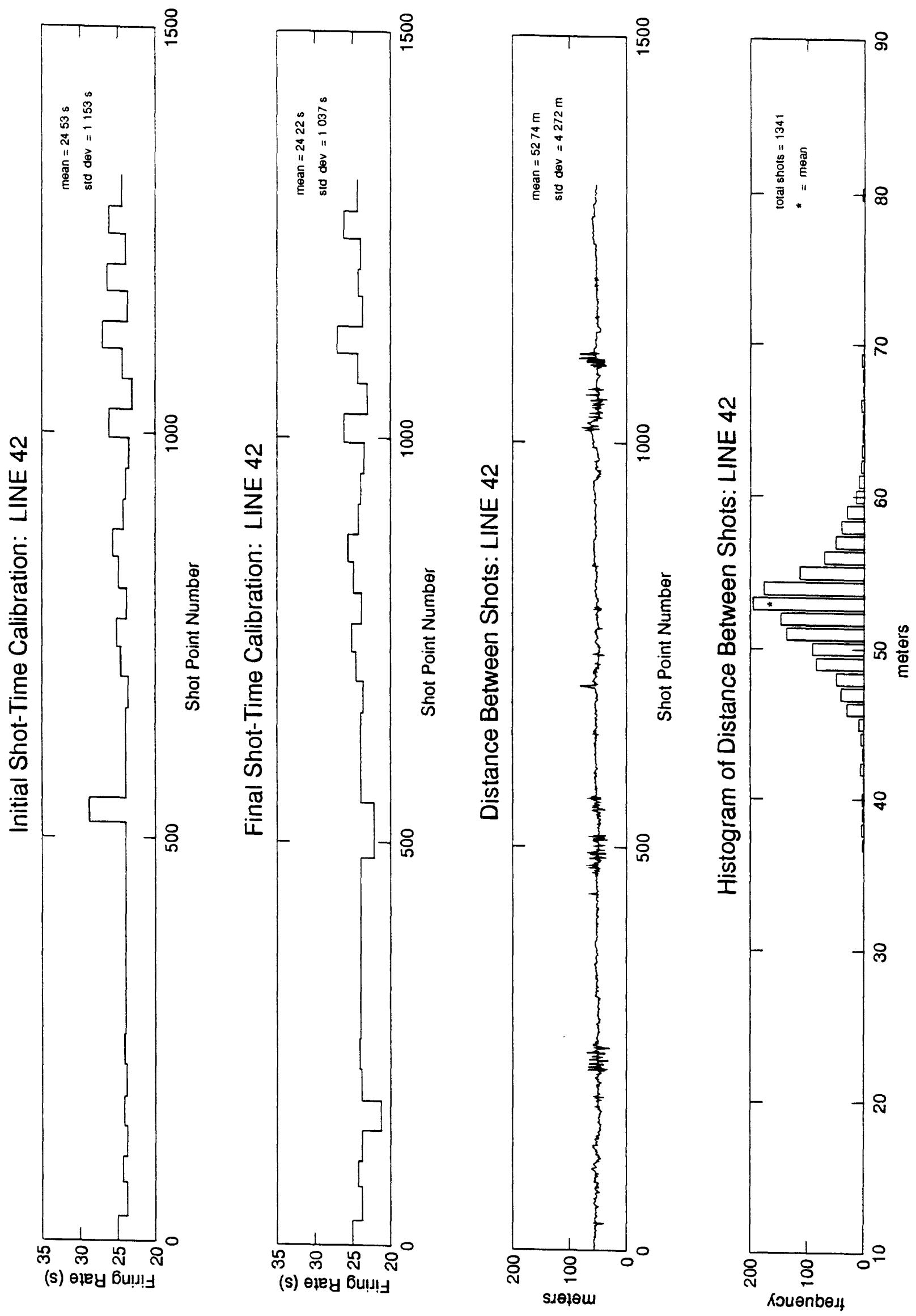

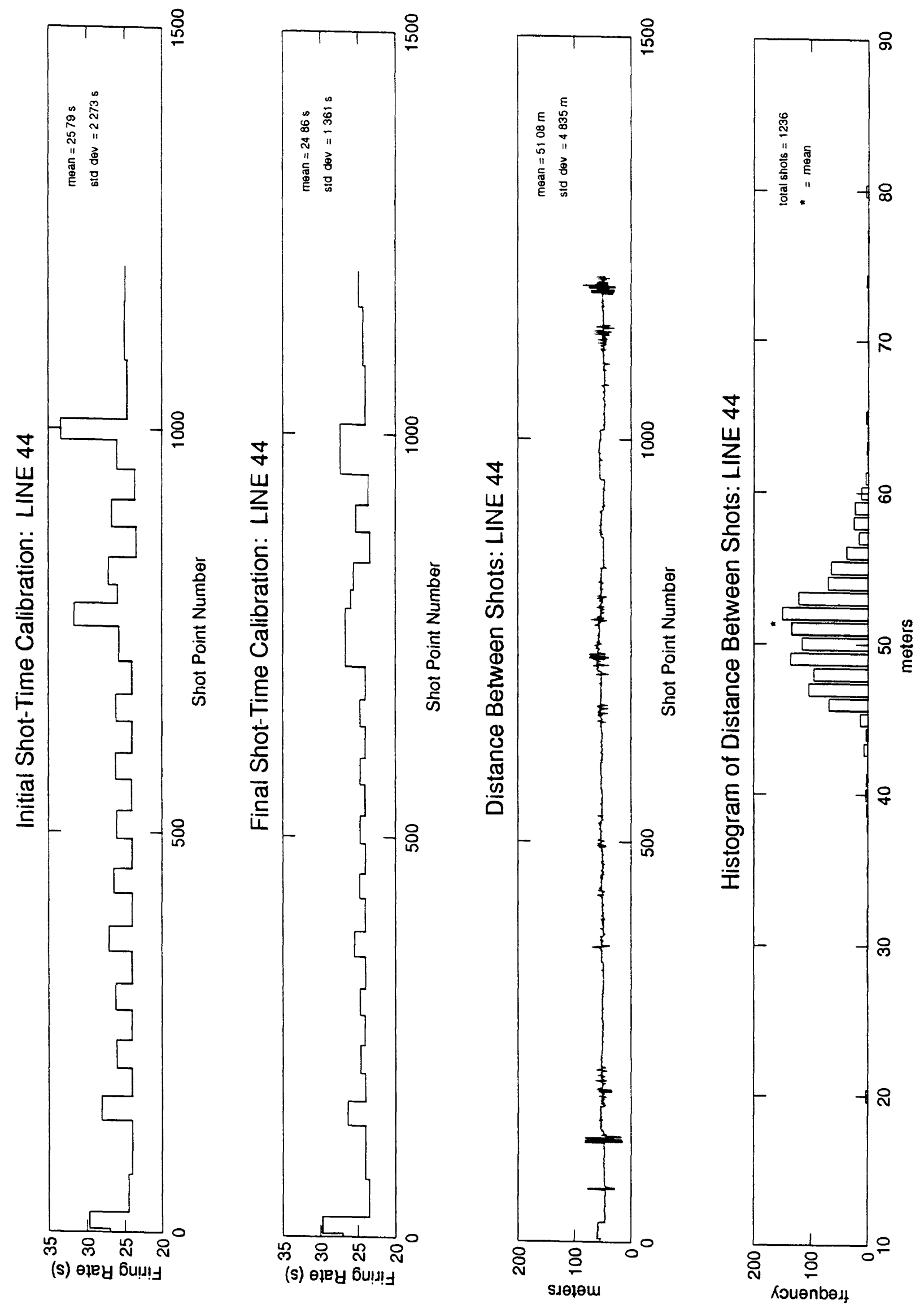


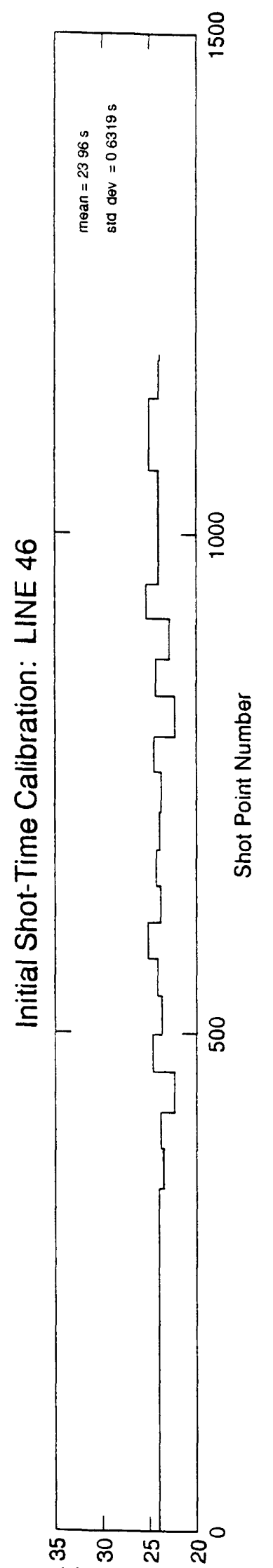

(s) өley 6u!!」

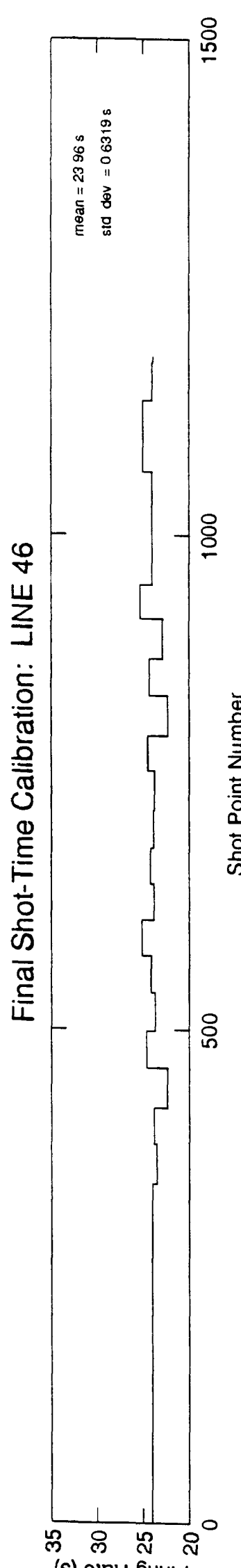

(s) oley bu!n!
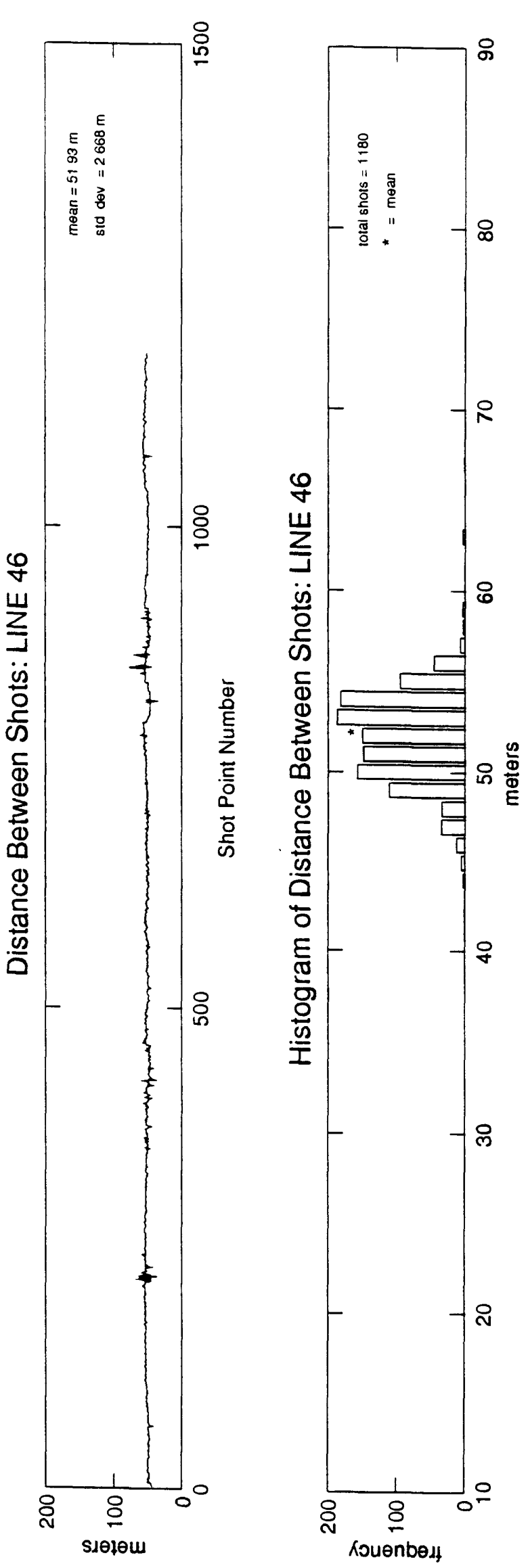

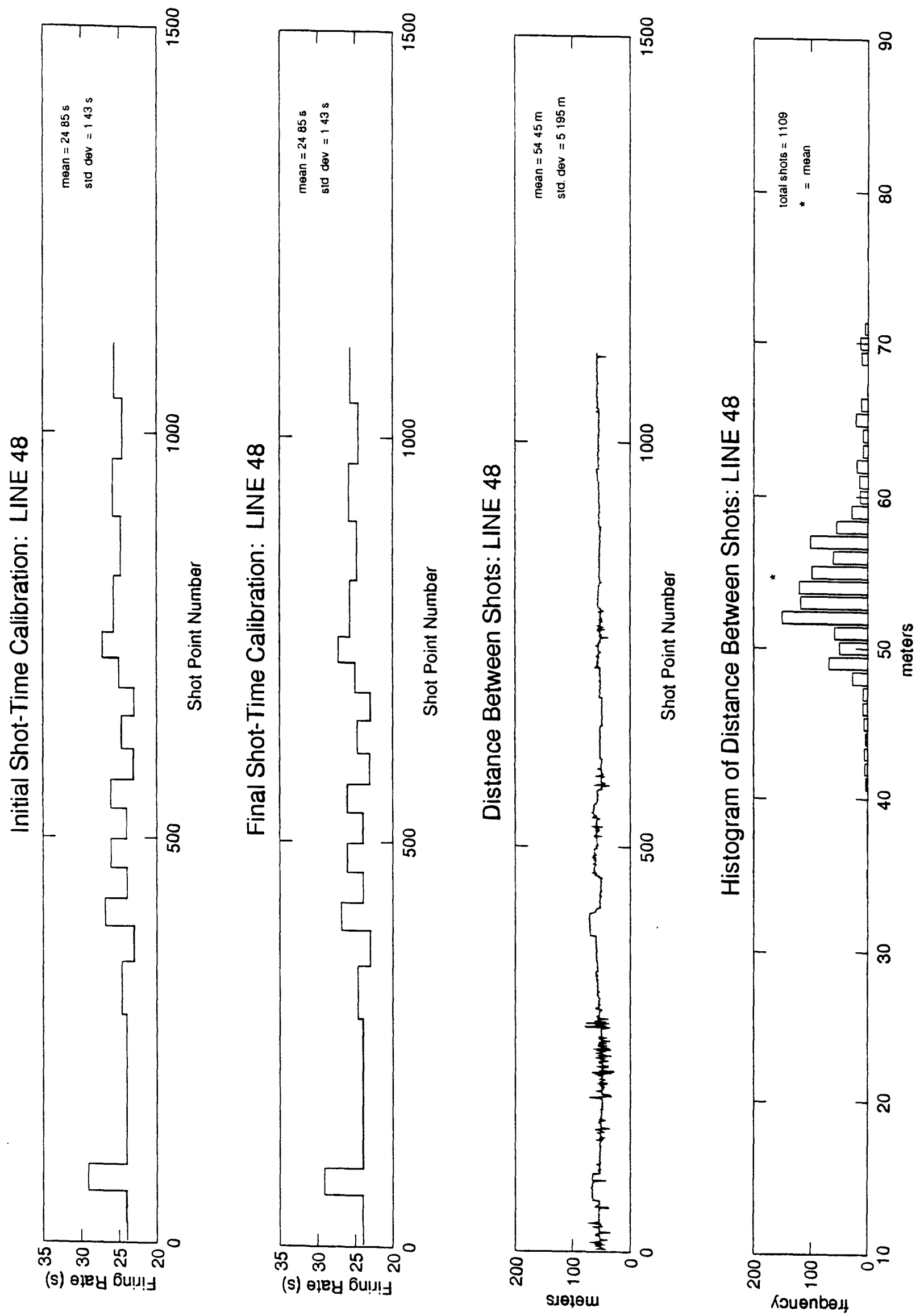


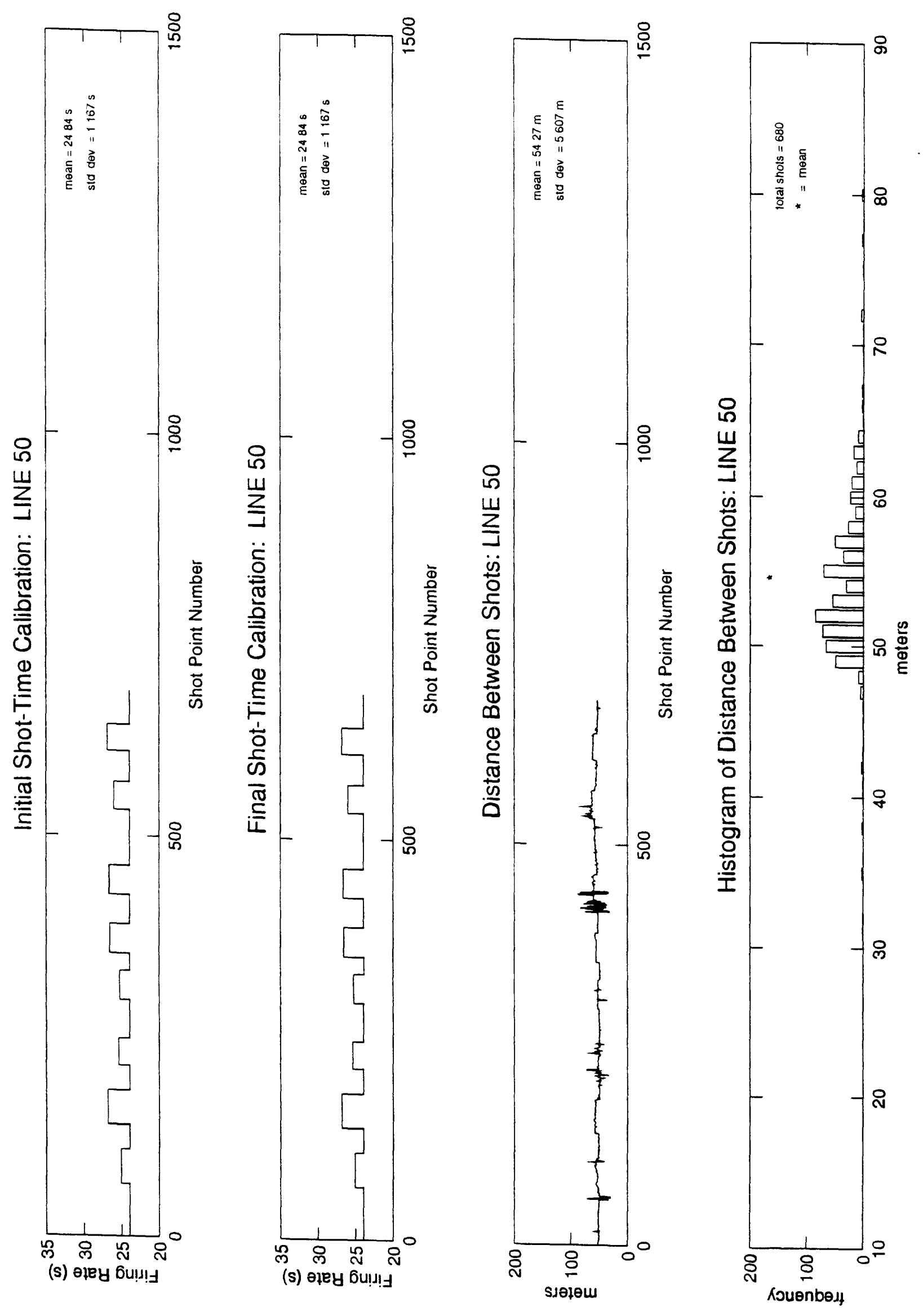




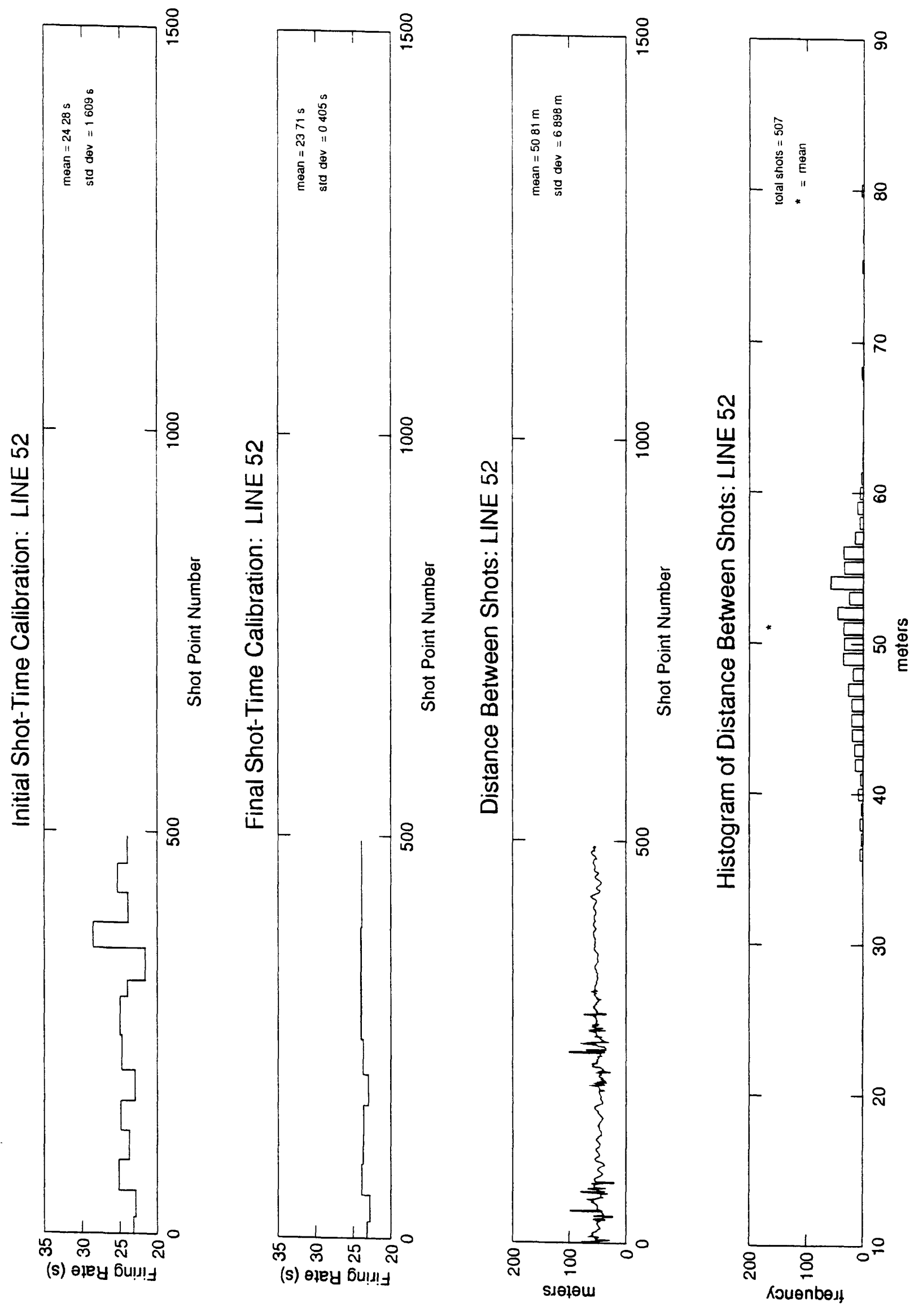



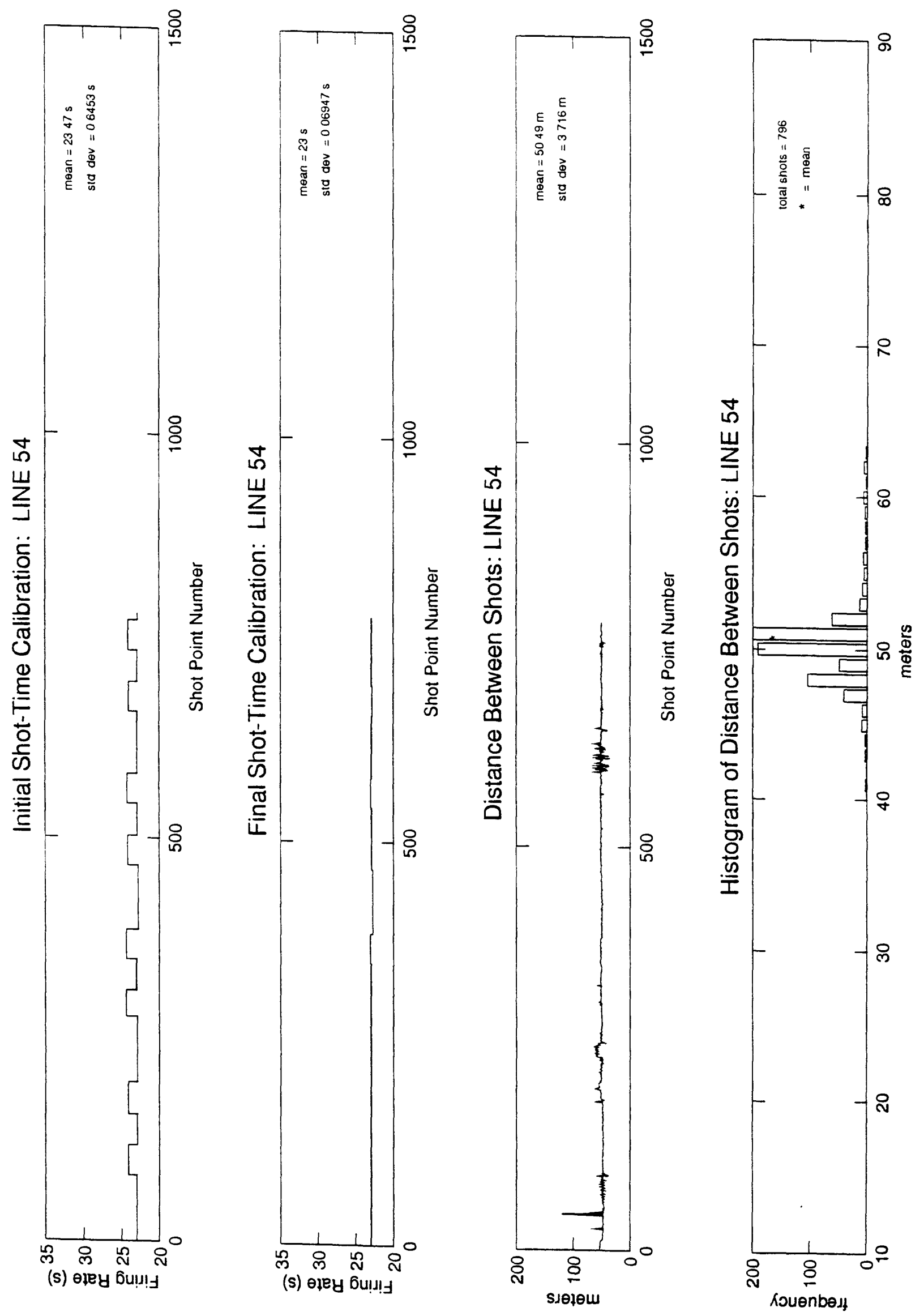

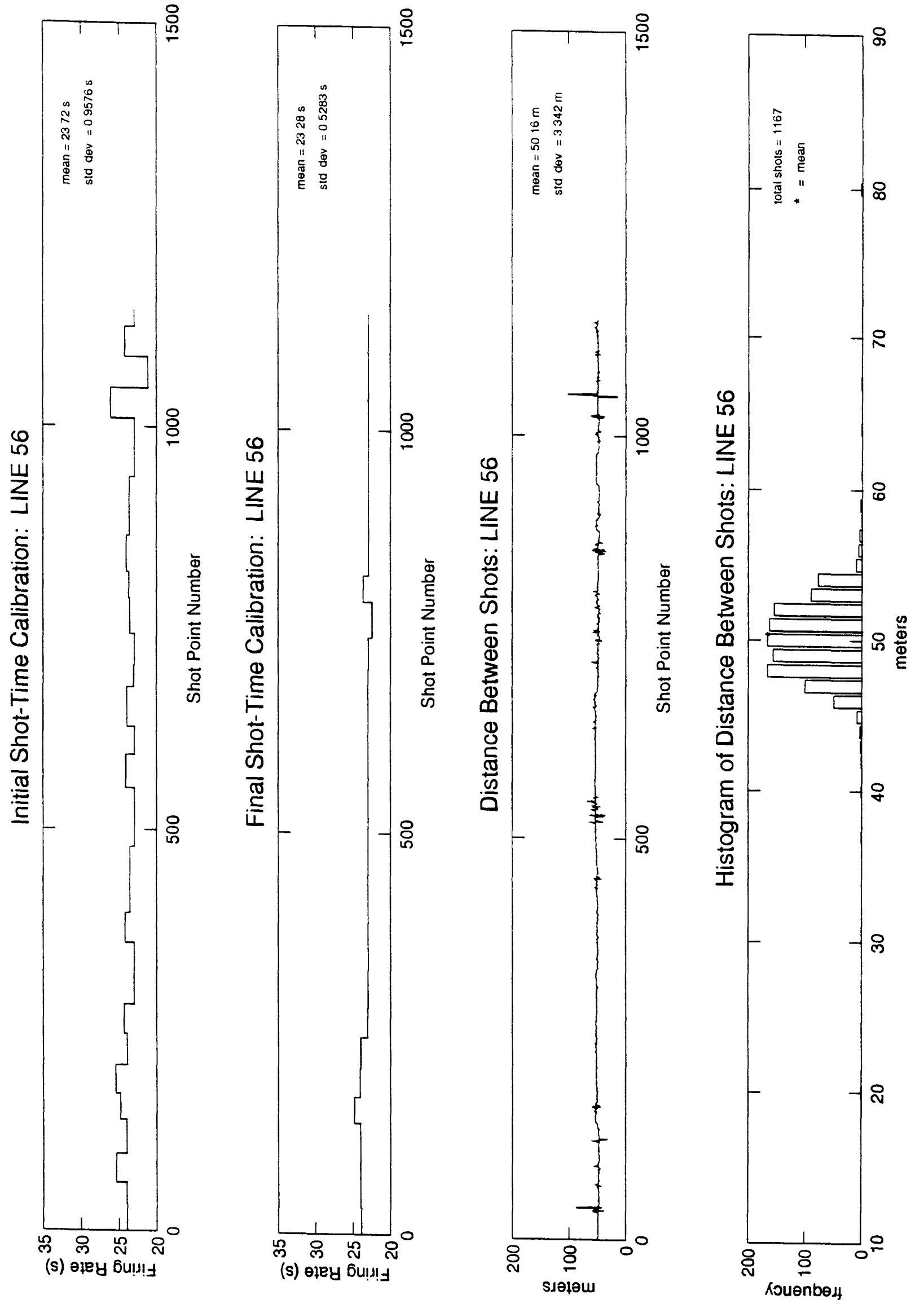

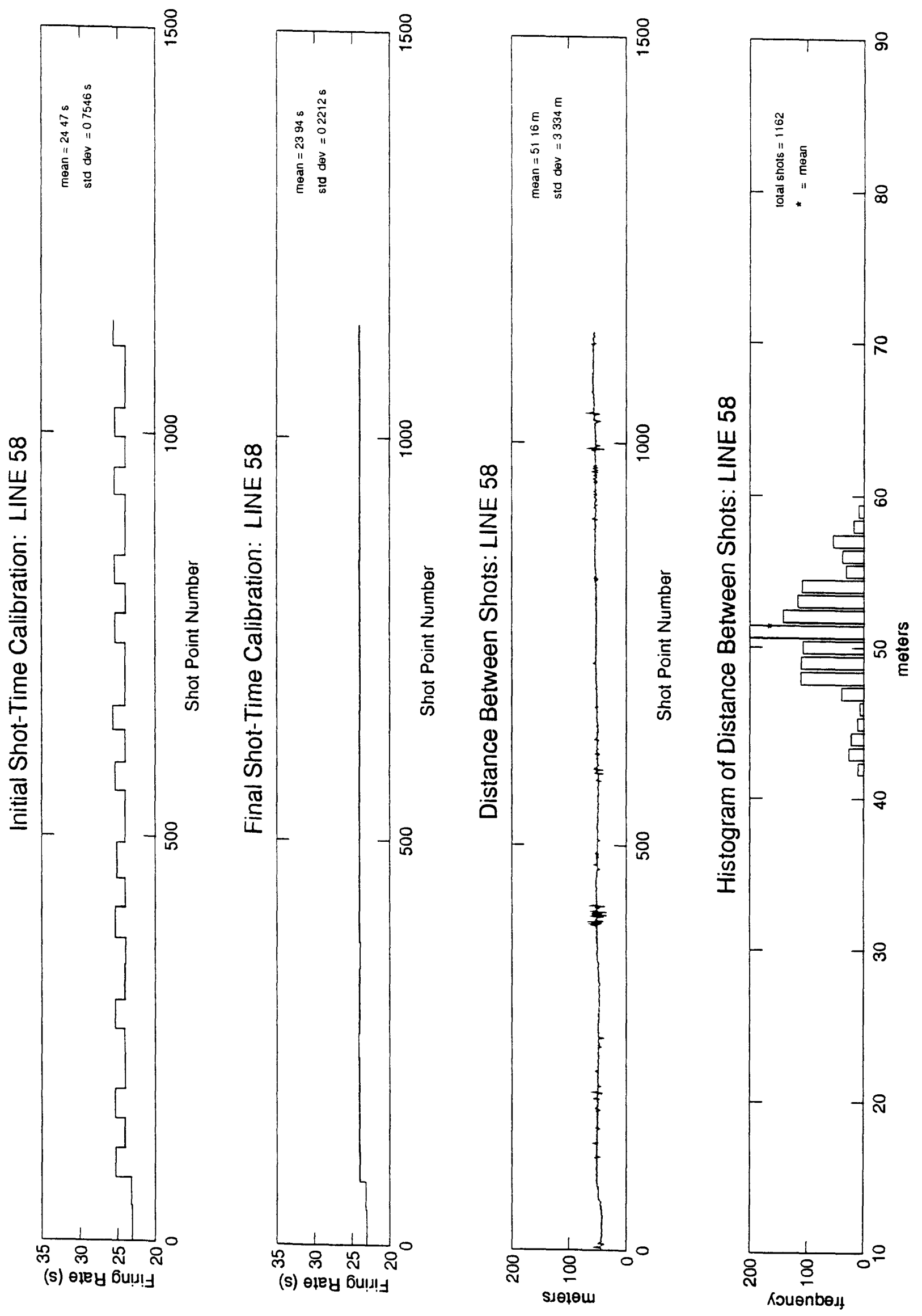

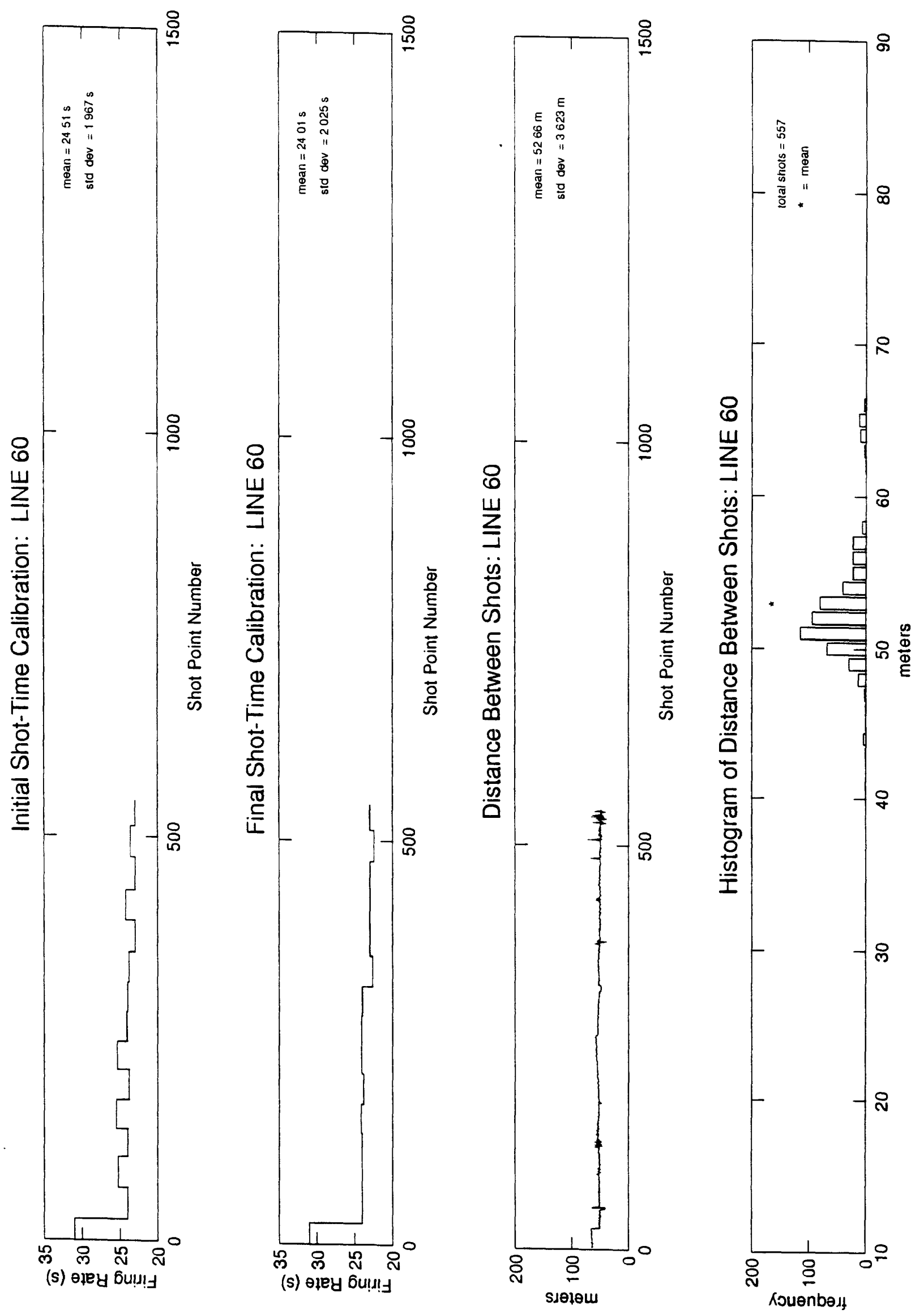


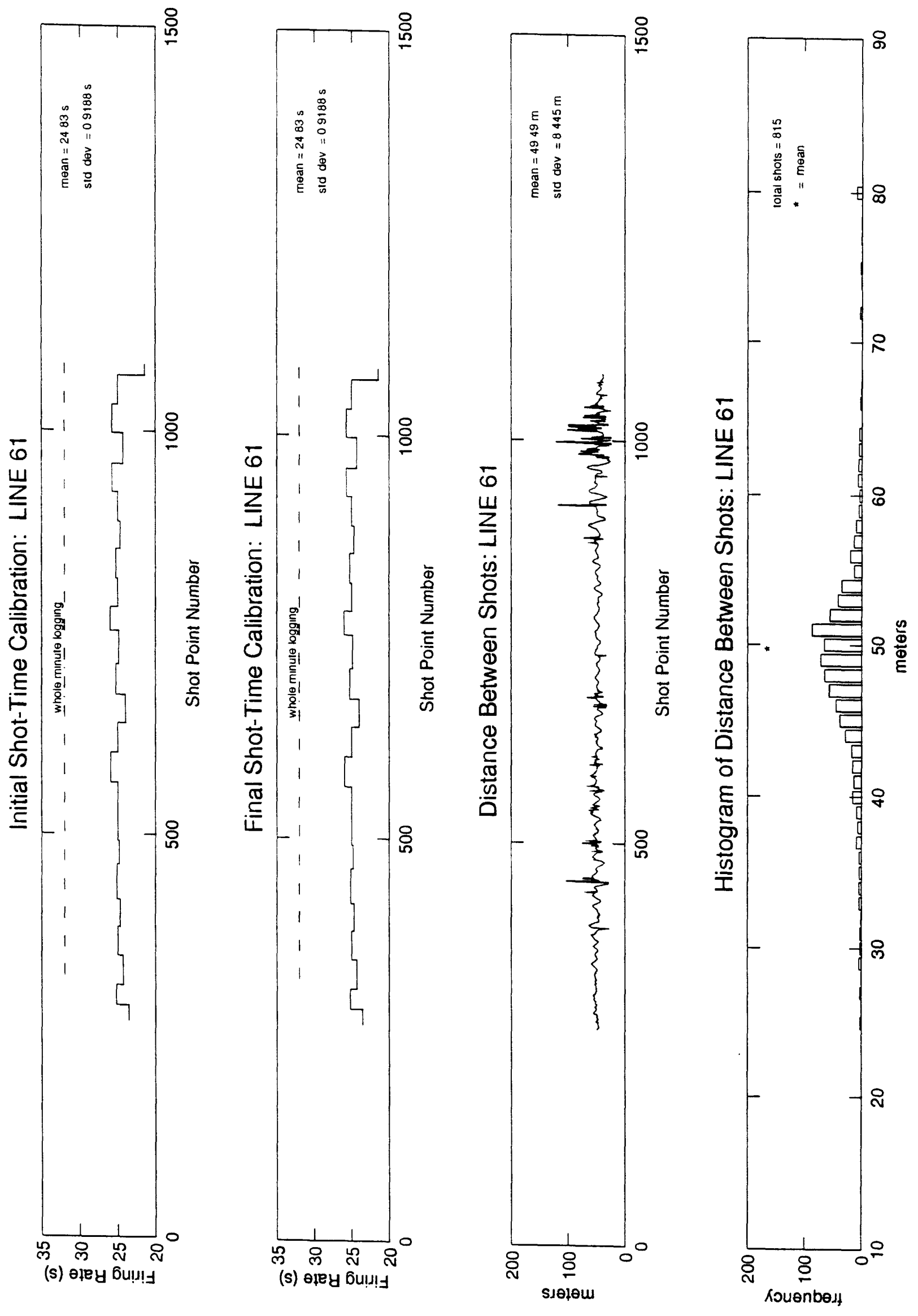




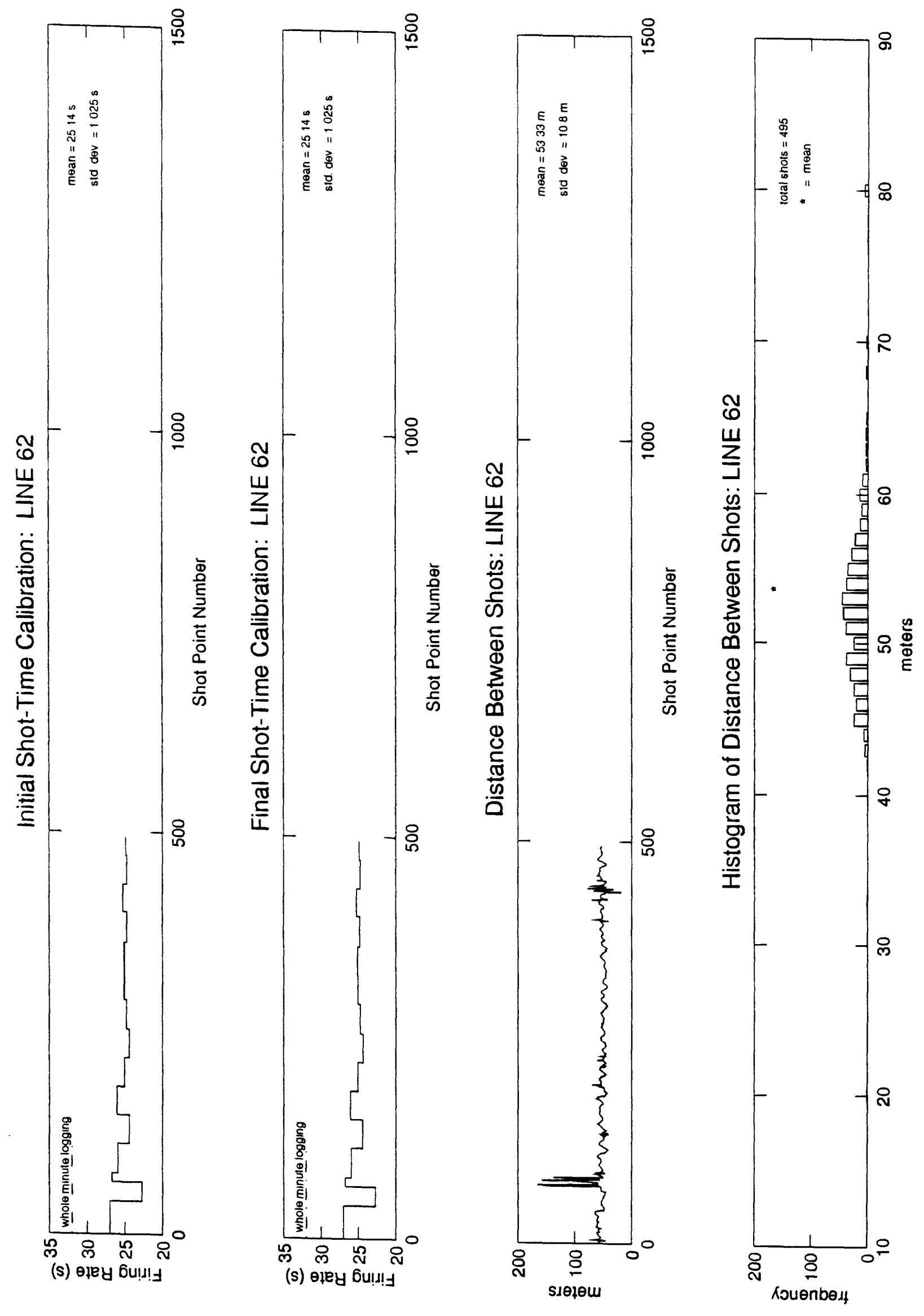



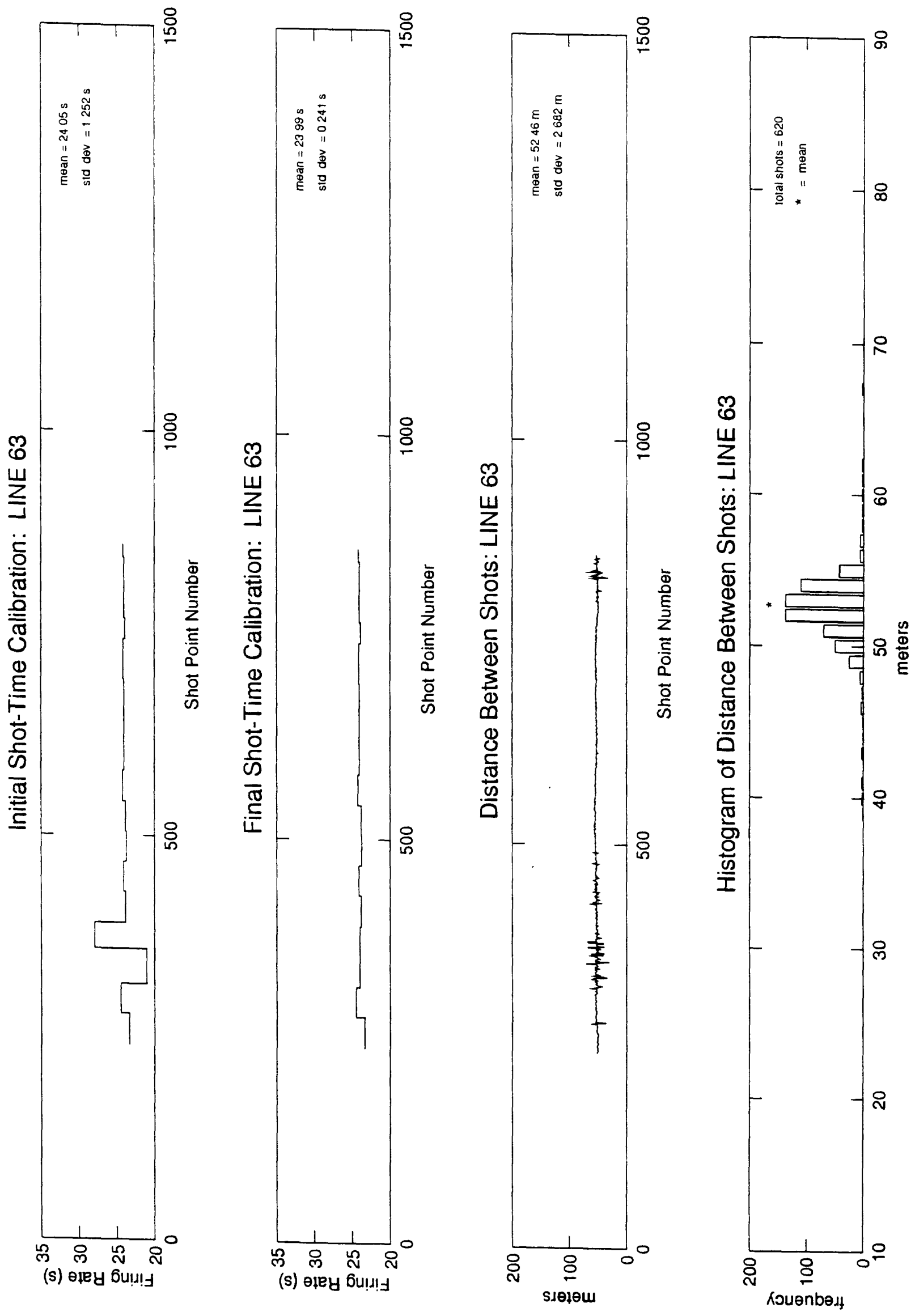


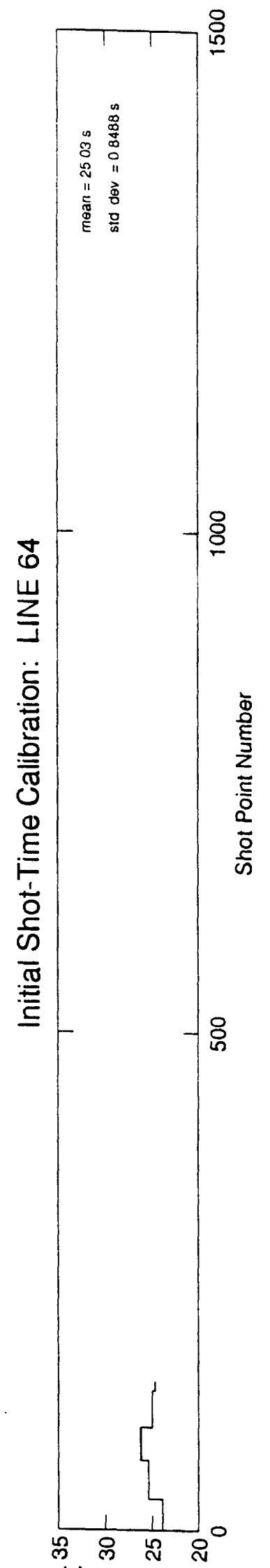

(s) өiey bu!n!
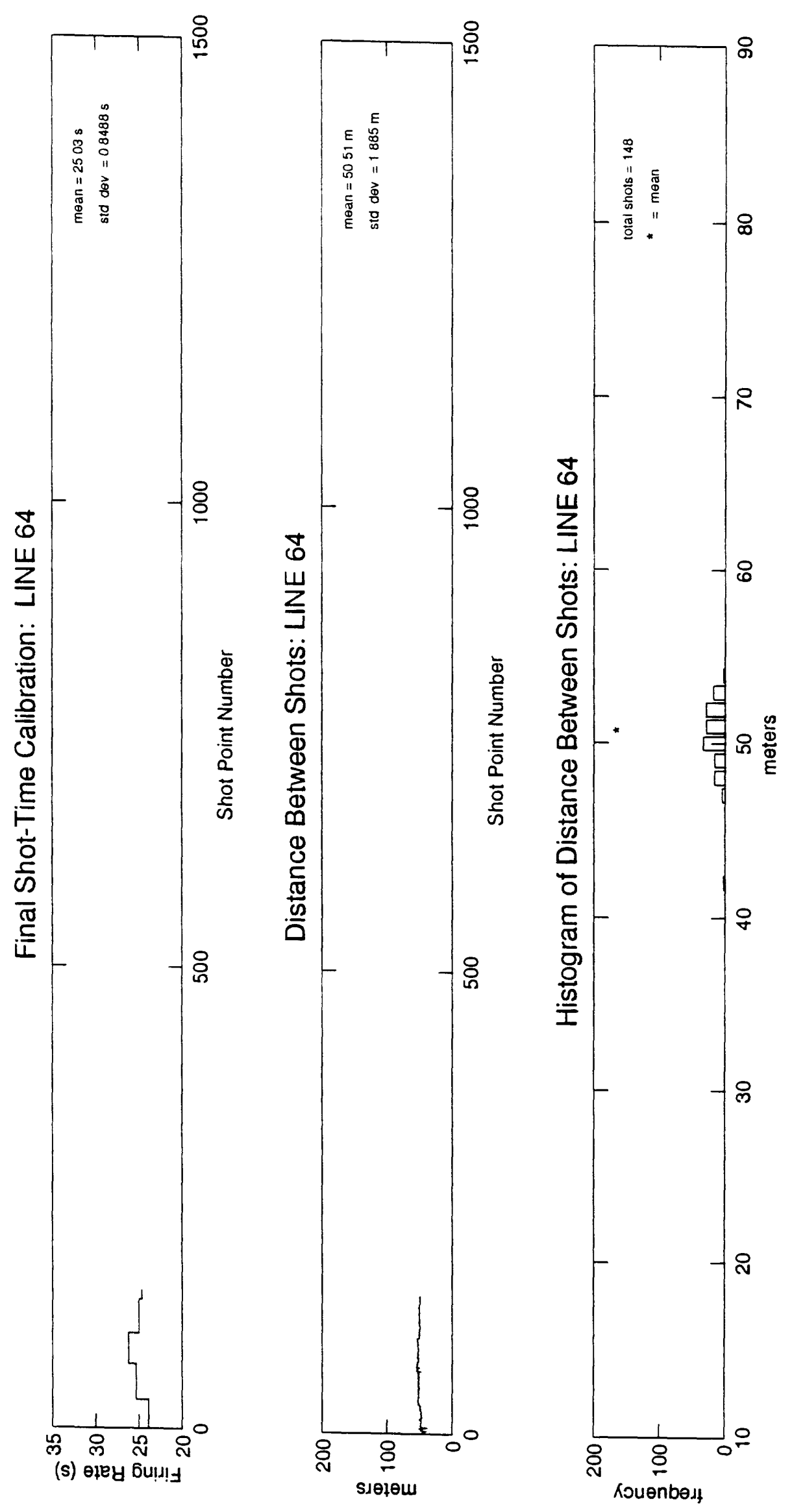


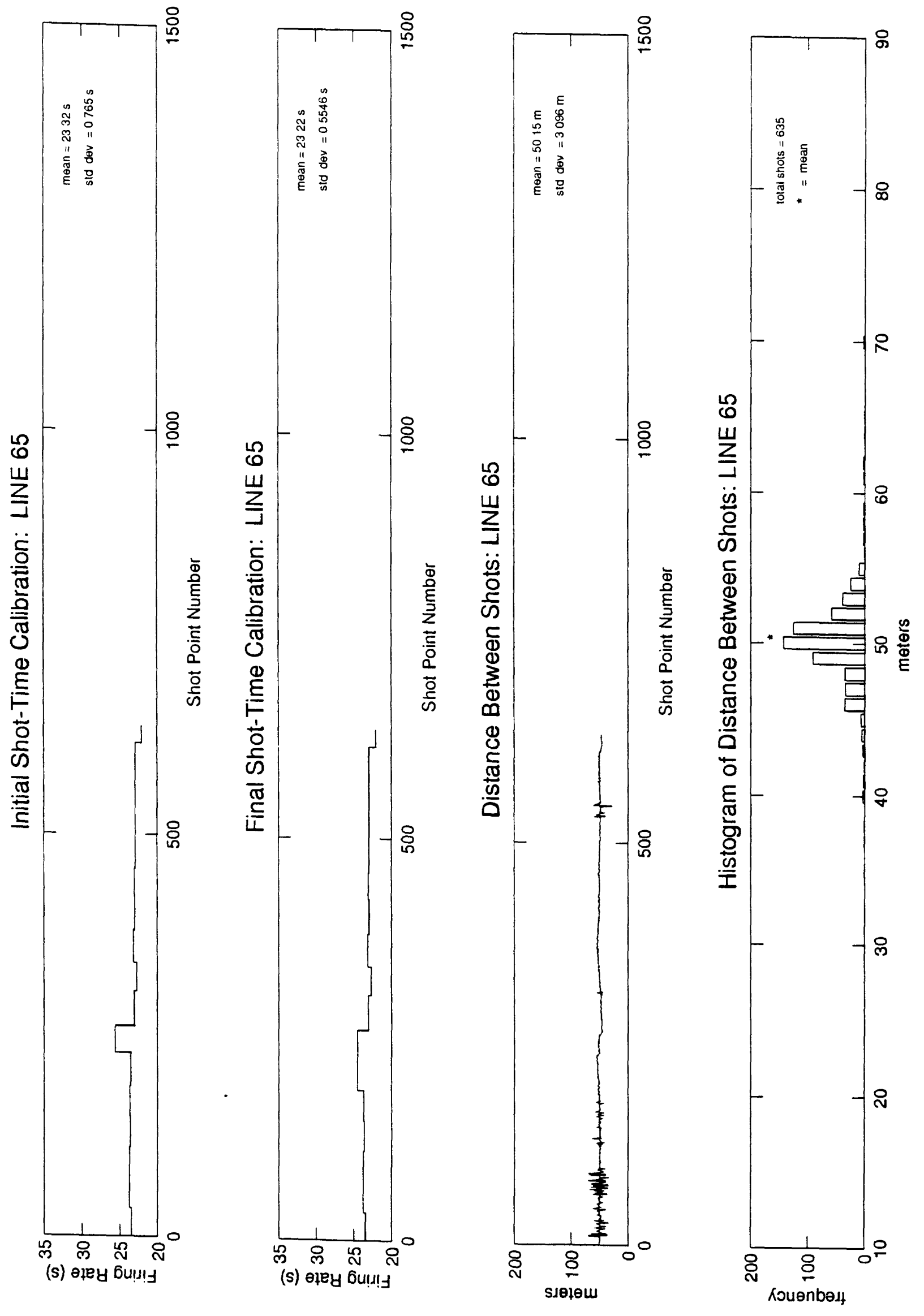




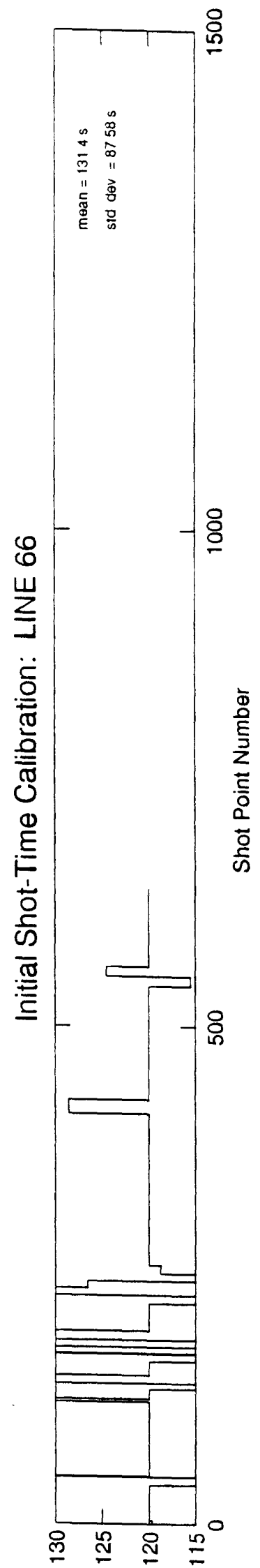

(s) өley bu!n!
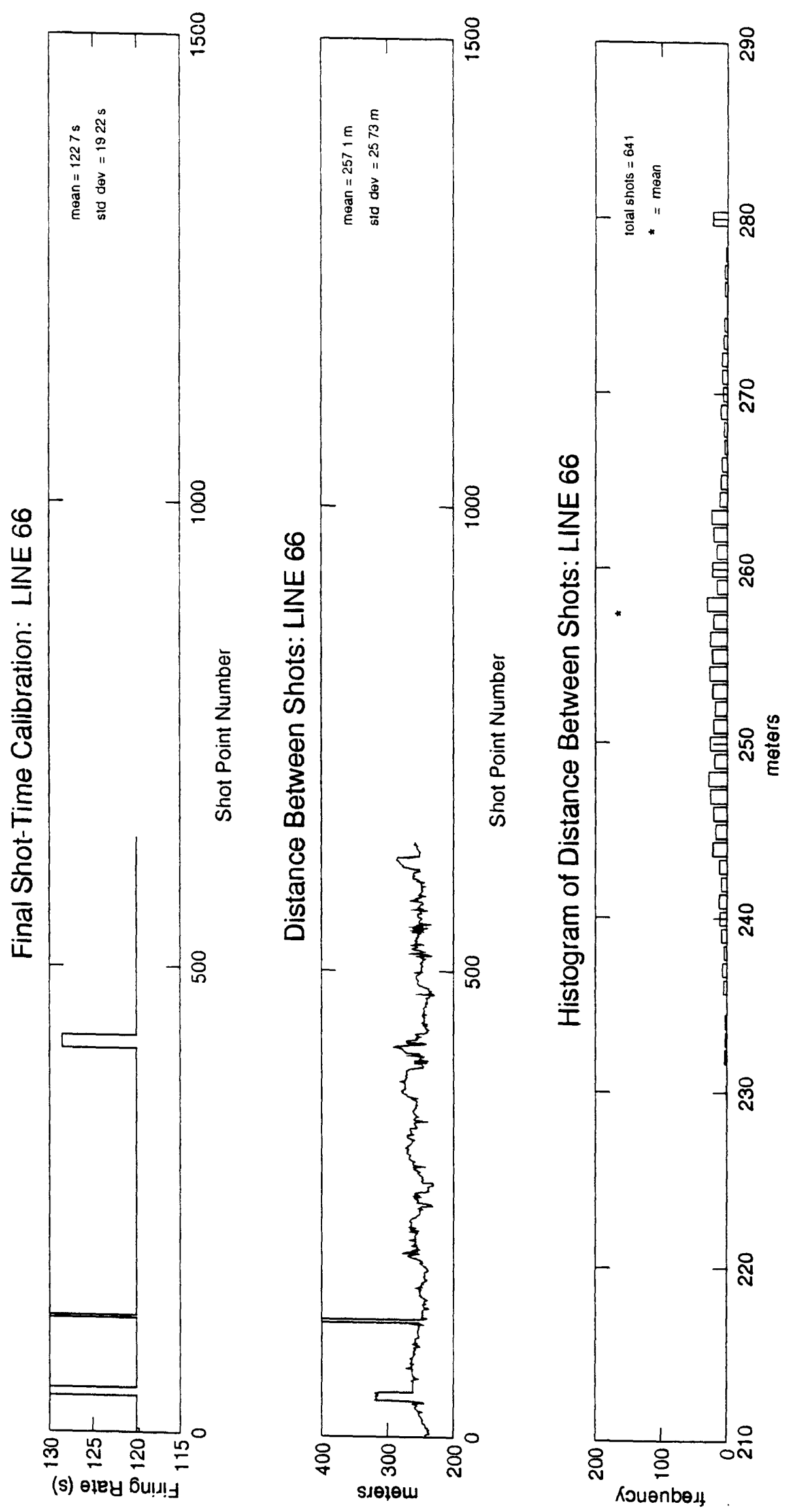


\section{APPENDIX 3}

\section{LINE CROSSING INFORMATION}


TABLE A1: LINE CROSSINGS

\begin{tabular}{|c|c|c|c|}
\hline Line No. & Shot No. & Line No. & Shot No. \\
\hline 2 & 199 & 3 & 166 \\
\hline 2 & 462 & 7 & 2042 \\
\hline 3 & 166 & 2 & 199 \\
\hline 3 & 345 & 4 & 539 \\
\hline 4 & 96 & 7 & 1777 \\
\hline 4 & 271 & 26 & 445 \\
\hline 4 & 539 & 3 & 345 \\
\hline 4 & 540 & $62 / 3 \mathrm{~A}$ & 77 \\
\hline 6 & 80 & 25 & 1329 \\
\hline 6 & 231 & $62 / 3 \mathrm{~A}$ & 238 \\
\hline 6 & 280 & 26 & 189 \\
\hline 6 & 814 & 28 & 161 \\
\hline 6 & 822 & 7 & 1525 \\
\hline 6 & 946 & 11 & 2137 \\
\hline 7 & 45 & 24 & 40 \\
\hline 7 & 147 & 22 & 378 \\
\hline 7 & 321 & 20 & 17 \\
\hline 7 & 453 & 18 & 354 \\
\hline 7 & 639 & 16 & 39 \\
\hline 7 & 763 & 14 & 367 \\
\hline 7 & 901 & 12 & 89 \\
\hline 7 & 995 & 25 & 154 \\
\hline 7 & 1141 & $61 / 10 \mathrm{~A}$ & 897 \\
\hline 7 & 1200 & 30 & 108 \\
\hline 7 & 1291 & 11 & 1974 \\
\hline 7 & 1337 & 8 & 104 \\
\hline 7 & 1525 & 6 & 822 \\
\hline 7 & 1552 & 28 & 144 \\
\hline 7 & 1777 & 4 & 96 \\
\hline 7 & 1899 & 26 & 569 \\
\hline 7 & 2042 & 2 & 462 \\
\hline
\end{tabular}




\begin{tabular}{|c|c|c|c|}
\hline Line No. & Shot No. & Line No. & Shot No. \\
\hline 8 & 80 & 11 & 2008 \\
\hline 8 & 104 & 7 & 1337 \\
\hline 8 & 256 & 28 & 291 \\
\hline 8 & 611 & 25 & 947 \\
\hline 8 & 818 & $62 / 3 A$ & 363 \\
\hline 8 & 929 & 26 & 8 \\
\hline 9 & 127 & $61 / 10 \mathrm{~A}$ & 1070 \\
\hline 10 & 607 & 25 & 486 \\
\hline 11 & 170 & 34 & 183 \\
\hline 11 & 388 & 32 & 370 \\
\hline 11 & 546 & 52 & 133 \\
\hline 11 & 745 & 24 & 281 \\
\hline 11 & 905 & 22 & 153 \\
\hline 11 & 1034 & 20 & 259 \\
\hline 11 & 1209 & 18 & 105 \\
\hline 11 & 1373 & 16 & 299 \\
\hline 11 & 1535 & 14 & 99 \\
\hline 11 & 1638 & 12 & 292 \\
\hline 11 & 1826 & 25 & 317 \\
\hline 11 & 1879 & $61 / 10 \mathrm{~A}$ & 815 \\
\hline 11 & 1882 & 30 & 63 \\
\hline 11 & 1974 & 7 & 1291 \\
\hline 11 & 2008 & 8 & 80 \\
\hline 11 & 2137 & 6 & 946 \\
\hline 12 & 15 & 25 & 13 \\
\hline 12 & 89 & 7 & 901 \\
\hline 12 & 292 & 11 & 1638 \\
\hline 13 & 282 & $65 / 50 \mathrm{~B}$ & 409 \\
\hline 13 & 411 & 48 & 136 \\
\hline 13 & 668 & 46 & 208 \\
\hline 13 & 883 & 44 & 907 \\
\hline
\end{tabular}




\begin{tabular}{|c|c|c|c|}
\hline Line No. & Shot No. & Line No. & Shot No. \\
\hline 13 & 1078 & 42 & 460 \\
\hline 13 & 1234 & $63 / 40 \mathrm{~A}$ & 334 \\
\hline 13 & 1413 & 38 & 561 \\
\hline 13 & 2541 & 36 & 59 \\
\hline 14 & 99 & 11 & 1535 \\
\hline 14 & 367 & 7 & 763 \\
\hline 15 & 41 & 21 & 635 \\
\hline 15 & 43 & 42 & 800 \\
\hline 15 & 235 & 44 & 520 \\
\hline 15 & 447 & 46 & 679 \\
\hline 15 & 708 & 48 & 654 \\
\hline 15 & 1002 & 50 & 354 \\
\hline 15 & 1307 & 54 & 476 \\
\hline 15 & 1743 & 56 & 581 \\
\hline 15 & 1938 & 58 & 565 \\
\hline 16 & 39 & 7 & 639 \\
\hline 16 & 299 & 11 & 1373 \\
\hline 17 & 313 & 42 & 1240 \\
\hline 17 & 496 & 44 & 101 \\
\hline 17 & 550 & 21 & 135 \\
\hline 17 & 702 & 46 & 1068 \\
\hline 17 & 971 & 48 & 990 \\
\hline 17 & 1278 & 50 & 58 \\
\hline 17 & 1568 & 54 & 226 \\
\hline 17 & 1871 & 56 & 793 \\
\hline 17 & 2076 & 58 & 389 \\
\hline 17 & 2245 & 60 & 64 \\
\hline 18 & 105 & 11 & 1209 \\
\hline 18 & 354 & 7 & 453 \\
\hline 19 & 63 & 32 & 33 \\
\hline 19 & 328 & 34 & 399 \\
\hline
\end{tabular}




\begin{tabular}{|c|c|c|c|}
\hline Line No. & Shot No. & Line No. & Shot No. \\
\hline 19 & 566 & 36 & 225 \\
\hline 20 & 17 & 7 & 321 \\
\hline 20 & 259 & 11 & 1034 \\
\hline 21 & 133 & $66 / 17 \mathrm{~A}$ & 550 \\
\hline 21 & 135 & 17 & 550 \\
\hline 21 & 248 & 44 & 202 \\
\hline 21 & 631 & 42 & 804 \\
\hline 21 & 635 & 15 & 41 \\
\hline 22 & 153 & 11 & 905 \\
\hline 22 & 378 & 7 & 147 \\
\hline 23 & 103 & 40 & 102 \\
\hline 24 & 40 & 7 & 45 \\
\hline 24 & 281 & 11 & 745 \\
\hline 25 & 13 & 12 & 15 \\
\hline 25 & 154 & 7 & 995 \\
\hline 25 & 317 & 11 & 1826 \\
\hline 25 & 376 & 30 & 18 \\
\hline 25 & 486 & 10 & 607 \\
\hline 25 & 490 & $61 / 10 \mathrm{~A}$ & 657 \\
\hline 25 & 563 & 28 & 408 \\
\hline 25 & 947 & 8 & 611 \\
\hline 25 & 1171 & $62 / 3 \mathrm{~A}$ & 289 \\
\hline 25 & 1185 & 26 & 124 \\
\hline 25 & 1329 & 6 & 80 \\
\hline 26 & 8 & 8 & 929 \\
\hline 26 & 124 & 25 & 1185 \\
\hline 26 & 137 & $62 / 3 \mathrm{~A}$ & 276 \\
\hline 26 & 189 & 6 & 280 \\
\hline 26 & 445 & 4 & 271 \\
\hline 26 & 569 & 7 & 1899 \\
\hline 28 & 144 & 7 & 1552 \\
\hline
\end{tabular}




\begin{tabular}{|c|c|c|c|}
\hline Line No. & Shot No. & Line No. & Shot No. \\
\hline 28 & 161 & 6 & 814 \\
\hline 28 & 291 & 8 & 256 \\
\hline 28 & 408 & 25 & 563 \\
\hline 30 & 18 & 25 & 376 \\
\hline 30 & 60 & $61 / 10 \mathrm{~A}$ & 811 \\
\hline 30 & 63 & 11 & 1882 \\
\hline 30 & 108 & 7 & 1200 \\
\hline 32 & 33 & 19 & 63 \\
\hline 32 & 370 & 11 & 388 \\
\hline 34 & 183 & 11 & 170 \\
\hline 34 & 399 & 19 & 328 \\
\hline 36 & 59 & 13 & 2541 \\
\hline 36 & 225 & 19 & 566 \\
\hline 38 & 561 & 13 & 1413 \\
\hline 40 & 102 & 23 & 103 \\
\hline 42 & 460 & 13 & 1078 \\
\hline 42 & 800 & 15 & 43 \\
\hline 42 & 804 & 21 & 631 \\
\hline 42 & 1240 & 17 & 313 \\
\hline 42 & 1240 & $66 / 17 \mathrm{~A}$ & 599 \\
\hline 44 & 99 & $66 / 17 \mathrm{~A}$ & 561 \\
\hline 44 & 101 & 17 & 496 \\
\hline 44 & 202 & 21 & 248 \\
\hline 44 & 520 & 15 & 235 \\
\hline 44 & 907 & 13 & 883 \\
\hline 46 & 208 & 13 & 668 \\
\hline 46 & 679 & 15 & 447 \\
\hline 46 & 1068 & 17 & 702 \\
\hline 46 & 1069 & $66 / 17 \mathrm{~A}$ & 517 \\
\hline 48 & 136 & 13 & 411 \\
\hline 48 & 654 & 15 & 708 \\
\hline
\end{tabular}




\begin{tabular}{|c|c|c|c|}
\hline Line No. & Shot No. & Line No. & Shot No. \\
\hline 48 & 990 & 17 & 971 \\
\hline 48 & 992 & $66 / 17 A$ & 460 \\
\hline 50 & 58 & 17 & 1278 \\
\hline 50 & 58 & $66 / 17 \mathrm{~A}$ & 401 \\
\hline 50 & 354 & 15 & 1002 \\
\hline 52 & 133 & 11 & 546 \\
\hline 54 & 226 & 17 & 1568 \\
\hline 54 & 226 & $66 / 17 \mathrm{~A}$ & 343 \\
\hline 54 & 476 & 15 & 1307 \\
\hline 56 & 581 & 15 & 1743 \\
\hline 56 & 791 & $66 / 17 A$ & 285 \\
\hline 56 & 793 & 17 & 1871 \\
\hline 58 & 389 & 17 & 2076 \\
\hline 58 & 390 & $66 / 17 \mathrm{~A}$ & 242 \\
\hline 58 & 565 & 15 & 1938 \\
\hline 60 & 64 & 17 & 2245 \\
\hline 60 & 65 & $66 / 17 \mathrm{~A}$ & 207 \\
\hline $61 / 10 A$ & 657 & 25 & 490 \\
\hline $61 / 10 \mathrm{~A}$ & 811 & 30 & 60 \\
\hline $61 / 10 \mathrm{~A}$ & 815 & 11 & 1879 \\
\hline $61 / 10 \mathrm{~A}$ & 897 & 7 & 1141 \\
\hline $61 / 10 \mathrm{~A}$ & 1070 & 9 & 127 \\
\hline $62 / 3 A$ & 77 & 4 & 540 \\
\hline $62 / 3 \mathrm{~A}$ & 238 & 6 & 231 \\
\hline $62 / 3 A$ & 276 & 26 & 137 \\
\hline $62 / 3 A$ & 289 & 25 & 1171 \\
\hline $62 / 3 \mathrm{~A}$ & 363 & 8 & 818 \\
\hline $63 / 40 \mathrm{~A}$ & 334 & 13 & 1234 \\
\hline $65 / 50 \mathrm{~B}$ & 409 & 13 & 282 \\
\hline $66 / 17 \mathrm{~A}$ & 207 & 60 & 65 \\
\hline $66 / 17 \mathrm{~A}$ & 242 & 58 & 390 \\
\hline
\end{tabular}




\begin{tabular}{||r|r|c|r|}
\hline Line No. & Shot No. & Line No. & Shot No. \\
\hline $66 / 17 \mathrm{~A}$ & 285 & 56 & 791 \\
\hline $66 / 17 \mathrm{~A}$ & 343 & 54 & 226 \\
\hline $66 / 17 \mathrm{~A}$ & 401 & 50 & 58 \\
\hline $66 / 17 \mathrm{~A}$ & 460 & 48 & 992 \\
\hline $66 / 17 \mathrm{~A}$ & 517 & 46 & 1069 \\
\hline $66 / 17 \mathrm{~A}$ & 550 & 21 & 133 \\
\hline $66 / 17 \mathrm{~A}$ & 561 & 44 & 99 \\
\hline $66 / 17 \mathrm{~A}$ & 599 & 42 & 1240 \\
\hline
\end{tabular}

